$* / 2 \%$ / 9 \$7( 5[B $\$ 7+2 *(1[B 52-(\& 7$

PART THREE. SPECIFIC EXCRETED PATHOGENS: ENVIRONMENTAL AND EPIDEMIOLOGY ASPECTS

\title{
CRYPTOSPORIDIUM SPP.
}

Walter Betancourt

University of Arizona 7XFVRQ I8 QLMGGGUMN 


\section{Copyright:}

\section{cc) (1) (2) \\ BY SA}

7KIVSXEQFDMRQIVIDYDIDE,

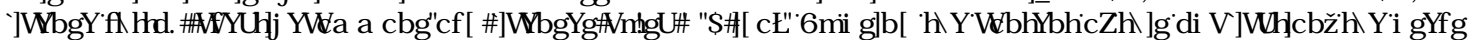

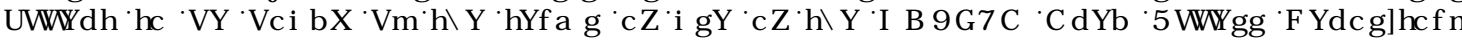

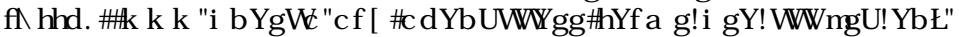

Disclaimer:

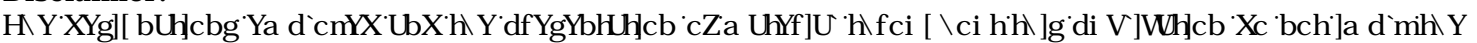

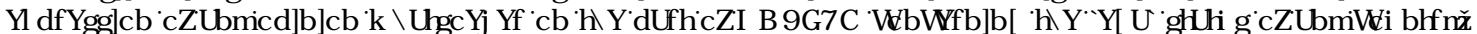

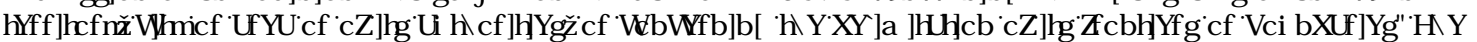

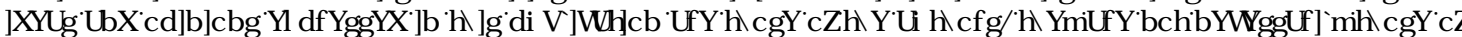
81 ( 6\&2 DQQGGRQRVIFRP P IMUKHR Y DQLIDURQ]

\section{Citation:}

Betancourt, W. (2019). Cryptosporidium spp. In: J.B. Rose and B. Jiménez-Cisneros (eds), Water and Sanitation for the 21st Century: Health and Microbiological Aspects of Excreta and Wastewater Management (Global Water Pathogen Project). (R. Fayer and W. Jakubowski (eds), Part 3: Specific Excreted Pathogens:

Environmental and Epidemiology Aspects - Section 3: Protists), Michigan State University, E. Lansing, MI, UNESCO. https://doi.org/10.14321/waterpathogens.33

Acknowledgements: K.R.L. Young, Project Design editor; Website Design Agroknow $\square$ http:// www.agroknow.com)

Last published: 


\section{Summary}

Cryptosporidium[LVD] HQXVRISLRMWMUHFR QLHGDVD P DRRUFDXVHR GDUKRHDQICOHMUIFRQMEXVG [M] QULFDQND

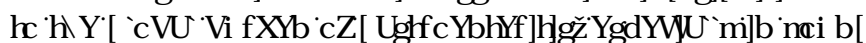

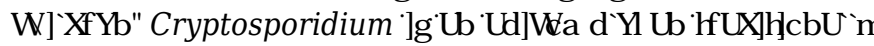
FRQVGHUHG DFRFFLGDQEXXVIVIP RUHFRQVHQ JHODVAG J HQHMFDQ [VR LMKH] UH DUQHM]Cryptosporidium [RFFXUV Z RUGZ LGHEXVIQ HFWRQLVLHSHFDC [SUHYDOHQWZK KHUH GUQNG ZZDMATXDOWDOGMDQLTWRQDUHSRRUIDQGIVP RWW

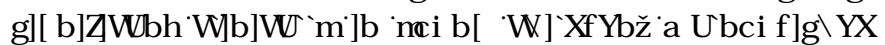
SHRSO1DOG[P P XQRFRP SURP LYHG \$SDHQW]

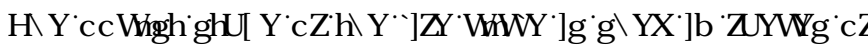
KXP DQVIDQGLDQP DQ/DQGLXXYLYHVP DQ THQYLRQP HQVDO FRQGWRQVDQGGUQ IFVDQW IR RFI WMKDYHEHQGGHFUALQ VXUDFHDOG] URXQG[Z DMAVIGUQNL] [ZDMAUZDMAZDMAV]

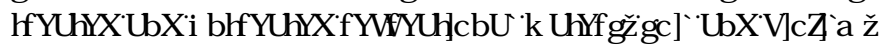

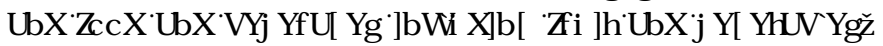

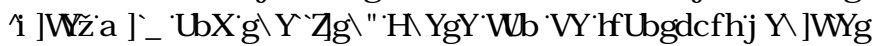

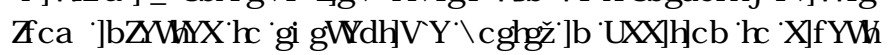
WDQMP IMRQYMLRXJ KSHURQJIRSHURQDQGDQP DQFRQMFVW

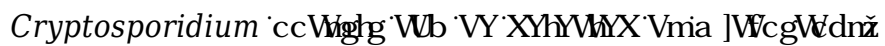

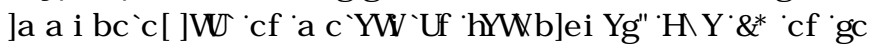
VSHFLMFDQRQQ[EHGHIHHQWDMAEI IP ROFXQUUYFKQTXHN

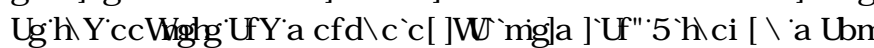

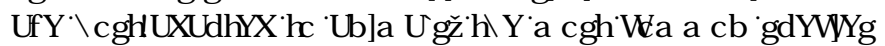
LQI HFWQ] KXP DQVDDH[C. hominis $\square$ C. parvum DQG $C$.

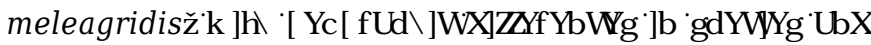
VXEWSH GLWUEXVRQT] HSLLA [GHFUSWRQ RI [MHYHUDO J HQRWSLQ [P HMRGVTMKHUHILVRRLWDQGDUGMHGP HMRGRI

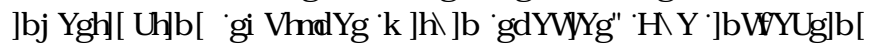
DYDCDELOWIR [MHTXHQFH GOMDDQGZ KROM] HQRP HIMTXHQFHN

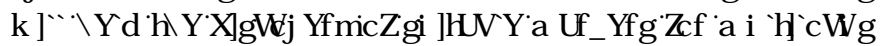
J HQRWSLQJ ITKKH [QP LWHG DDELOW IVR [SURSDJ DWH

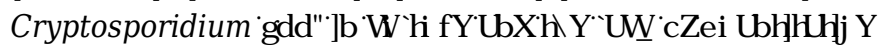
P ROFXQUIP HMRGVDRUDMHMP HQVRI [KXP DQRQI HFVYLW

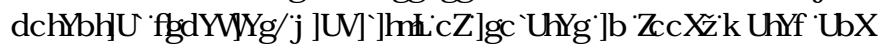

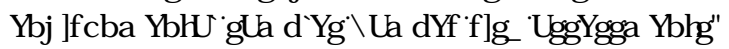

3UHYHQMRQDQGFRQWRQP HDVUHMLOFQGHSHURQDQKJ I HQH]

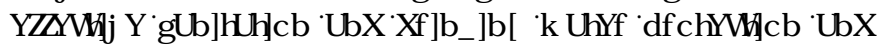

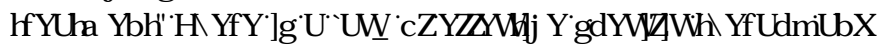
QRYPFFLOH

0 HP EHUR [YKH] HQXVCryptosporidium DUHRXNDU RWF

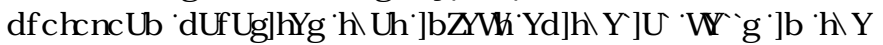
P IFURYLOXVERLGHIRIMKH] DURLQMMMQDQMDFVRIDQFCWMN RI [YHUAFUDMATCryptosporidium KDV[EHHQ_UFRI QLIHGDV RQH[RI LMKHP RWLRP P RQTHRQIJ IFDQD HQWRI ZZDMAERLOH GMHDY

\subsection{Epidemiology of the Disease and Pathogen(s)}

\subsection{Global Burden of Disease}

6LQFH[VKHLHP Hy HQFHRI Cryptosporidium DVDTKXP DQ

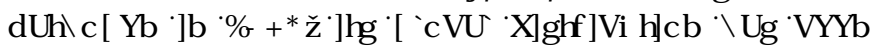

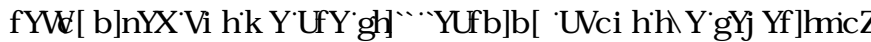

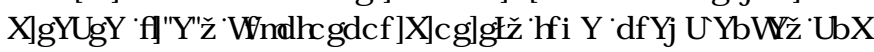

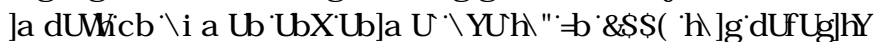

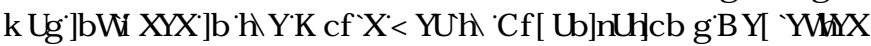

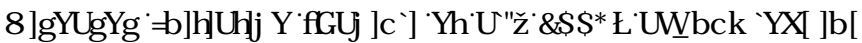

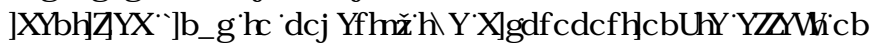

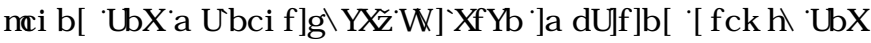

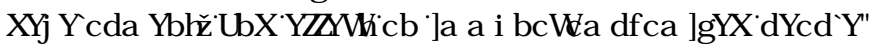
6WXGHDXQGHUNHQXVQ [P SURYHGGD QRWF DWM VKKDYH UHYHDOHG [MKDWVMKHTFRQWEXWRQRI [Cryptosporidium VR FKLQKRRGP RLELGLWDDQP RUDOWWVYMI QULFDQMDQGKDYH HWDEOMKHG[Cryptosporidium DP RQ [MKHP RWMP SRUDDQW LQ HFWRXVIFDXVHVRI P RGHDMALRLYHHUH GDUKRHDIZ LMK

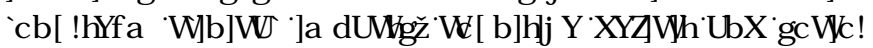

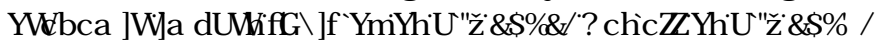
KDOQMDDQ

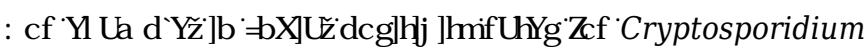

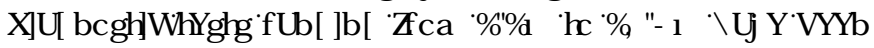
UHSRUAG[DP RQ] [SHRSO1[QFRP P XQLWHNDDQGDMAQGL] KHDOKFDH [MHMQ VWIR] HQHDMHDQQXDQQDMRQDOHMP DUAN

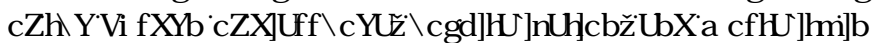

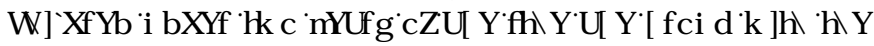

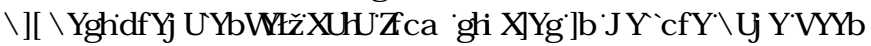

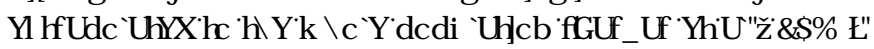

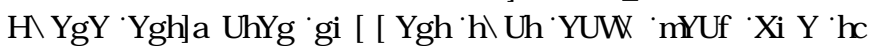

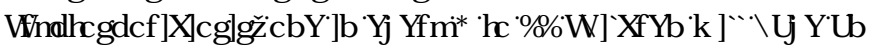

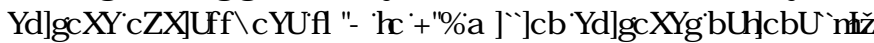

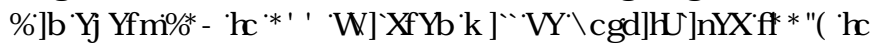

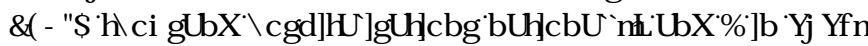

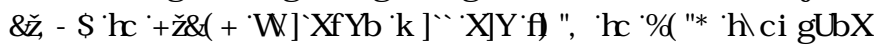

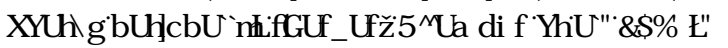

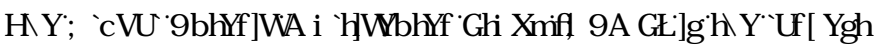
SLRVSHFWYHWXG RI GLUKRHDQP RLELGWDOGP RUVDONDQ

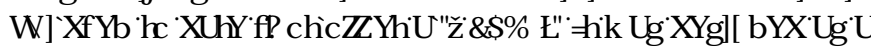

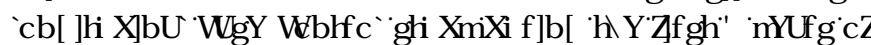

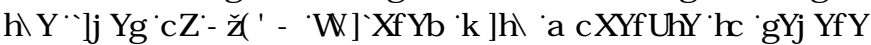

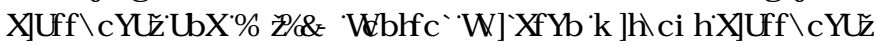

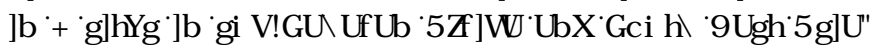

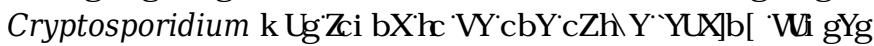

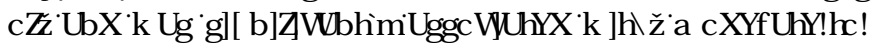

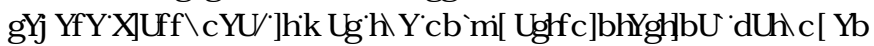

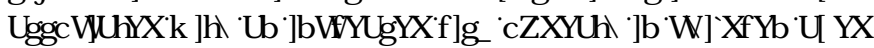

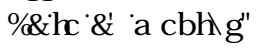

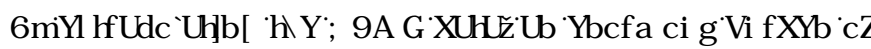

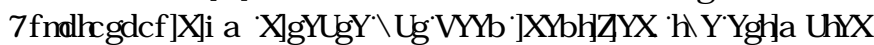
DQQXDQQXP EHURI Cryptosporidium DWWEXVIEOHGDUKRHD

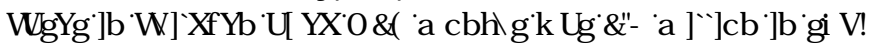

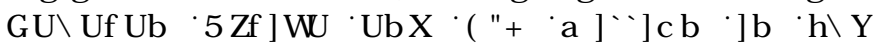

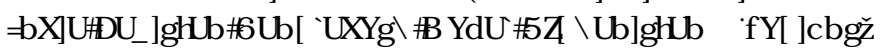

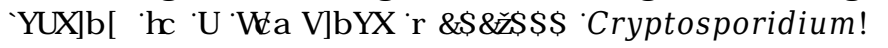

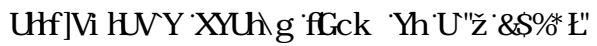

+RZHYHUTMKH] REDQMJ QULFDQFHRI FU SVRVSRUGRVVIV

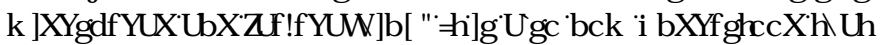

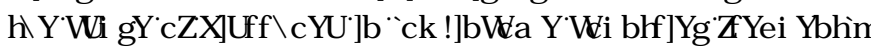
FRP SUMHMP XQUSOSSDMRI HQMZLLECryptosporidium DVRQH

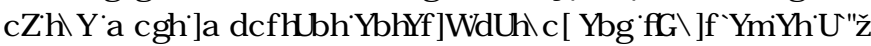

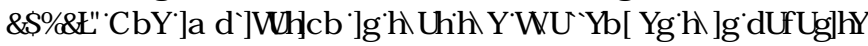

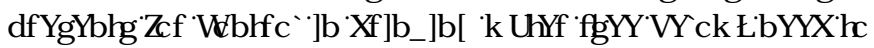


EHFRQVGHUHG [Z KHQQ HQHUDOFRQVROP HDVXUHVRU GDUKRHDQLOHWLDH[SXWLQTSOFH]

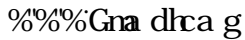

LUUKRHD TV TWKH P RWVFRP P RQ IM P SVRP RI FU SVRVSRUGRVYIEXVIVIP DQUHMLURQYYDUHMDFFLGQ ]YR

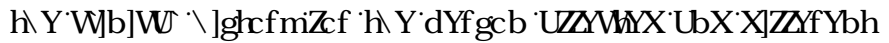

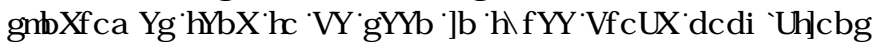
[\&KDSSHQOHQDQ ' DYHM四

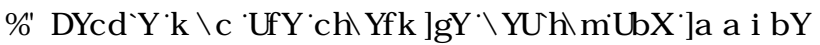
FRP SHMAQH SHUHQFHSLRRQ HGIZDMAY GODKRHD RI IVXGGHQRQVHVIDEGRP LQDOSDLQTRDXVHDLDG YRP LUQ IDQG [RZ] UDGH[HYHUTVKHMH[M P SVRP V

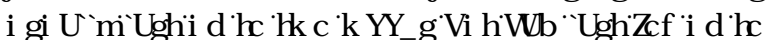

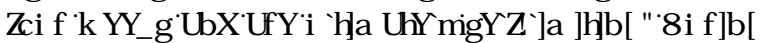

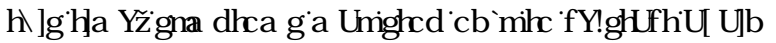

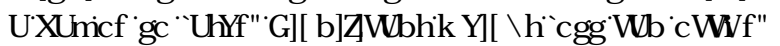

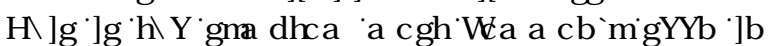
FKLGUHQDQGDGXQVПQР LGGOIDQG KL KПQFRP H FRXQNHM

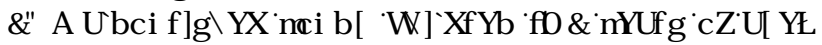
H[ SHUHQFHP RGHDMAIRR DMYHUHGDUKRHDTMKDUFDQ EHSHUMAQMDQGUHXQRQLLFUHDMGP RLELGWDQG

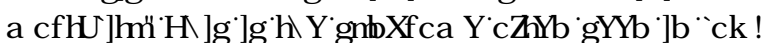
LOFRP HFRXQMHM

प] 6HYHUHQ ПP P XQRFRP SURP LMHG LQGLYLXDQ/P D VXIIHUFKURQIF LMHYHUHGLUKRHDRI QDU H[YROP H

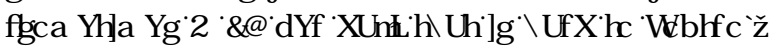

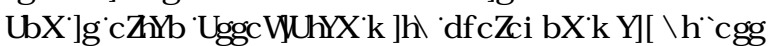
DQG [SURWWDMRQTDQG ROFUHDHGP RLELGW DQG

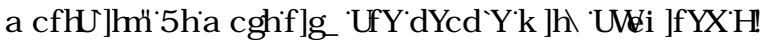

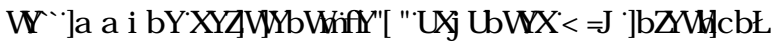

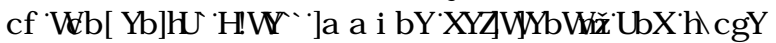
Z LVK [KHP DVROPJ IF PP DQJ QDQFUN IISDUMFXQDUQ

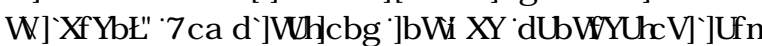
FU SVRVSRUGLRVVIZZ KLFK P DI JUUHQ [OIDG DVR VFOURYG [FKRODQ LUXDQGOYHUFWKRVYREVHUHGDQ SDWHQWIURP [QGXWWDD] HGFRXQWHMIBXФ RQDU FU SVRVRUGRYVIVIDORDUUHFRP SCFDURQ]

,QDDGGWRQCYR[VKH] DWRLQMAMQDQM QGURP H[XVXDCQ VHHQTSXФP RQDU TFU SVRVSRUGRVVLP DI EHDDQXXGHU] GL] QRVHG [GLVHDVH RQ FKLGUHQ Z LVK GGDUKRHD LQG

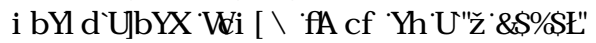

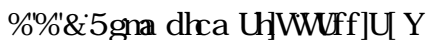

\$ $И$ P SWRP DWF TFDUULD H TRFFXUV LQQ TERWK IP P XQRFRP SHMHQWDQG IP P XQRFRP SURP LYHG [SDMHQW

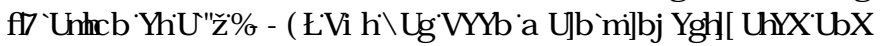

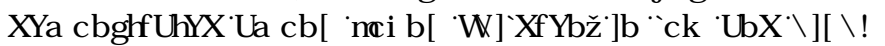
LQFRP HFRXQWHMT\$ P SVRP DUF FDUUD HP D [SUHMQVMD UMNRI RQZ DUG LWDQVP LWRQZZ KHUH[KIJ IHQH[V[SRRU] \&DUD HWDMAR VFWRQDQWXGHMRIP P XQRFRP SHMQNFKLGHQLQFKLCFDH

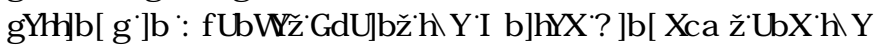

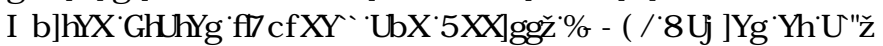

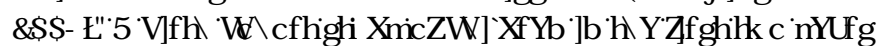

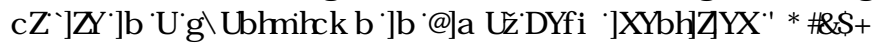

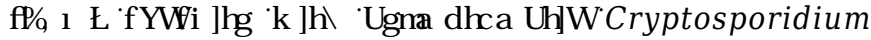
LQ HFWRQVIZ KLFK[ZHUHDRXQG]VREEHDMRFLDAGZZ LUK[SRRU

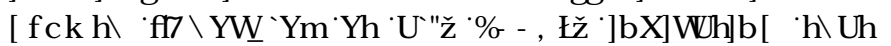
DU P SURP DMFLQ HFURQDORFDXYMDQIP SRUVDQMEXUGHQR GMHDY

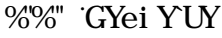

7KHFRQMTXHQFHMRIQHFURQZ LUECryptosporidium DH

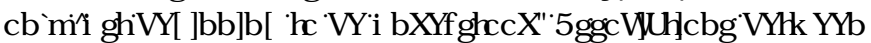
Cryptosporidium IP DOXXWWRQIDQG[GH IFLWLQTMKH] URZ WX

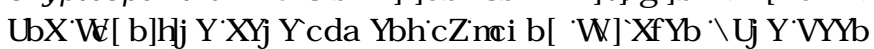
LGHQWU LHG [D \&KHFNOA LMDQ

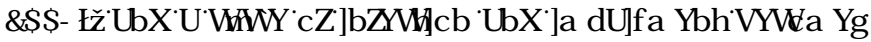

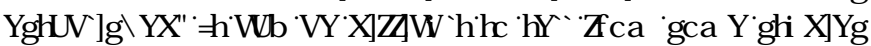

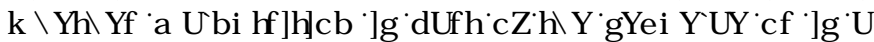

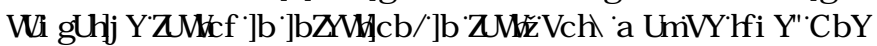

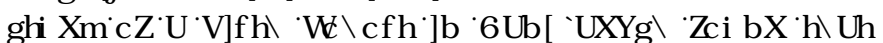

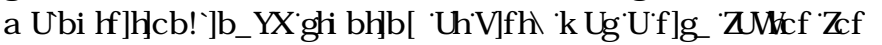

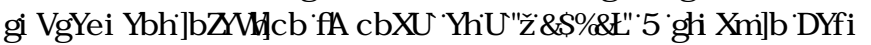

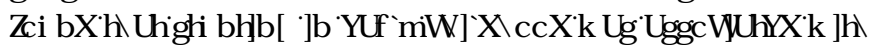

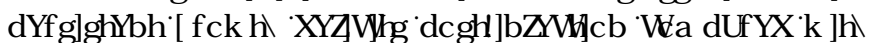
QRQ]WXQMGFKLQUHQLLZ KRP [SRWRQIFURQ] URZ WX [GHLFLW

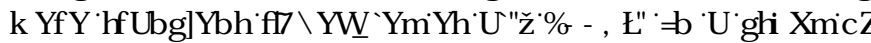

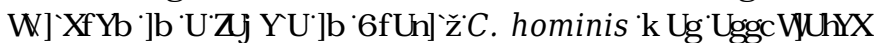

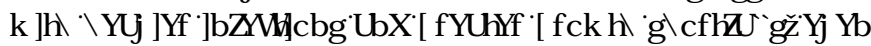

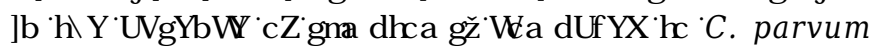

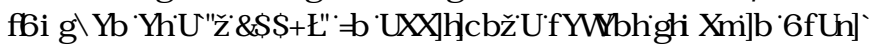

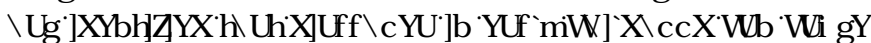

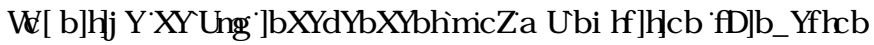

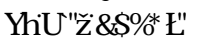

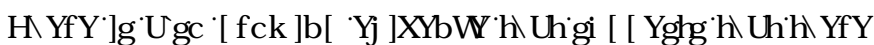
P D DDOREHQRQ HUMAP [KHDOK[HIIFWD WAUUHROWRQR DFXVAFU SURVSRUGRVYLQTL KДOFRP HFRXQMHMW KHUHDH

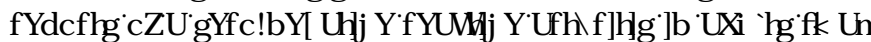

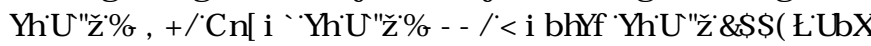
FKLQGHQW6KHSKHGLHMDQ

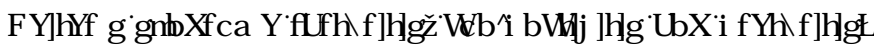
[\&URQDQG 6KHU IRXQGUMDVWFDHMRI FU SVRVSRUGRVYFDXVHGEI [C. hominis

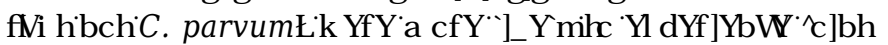

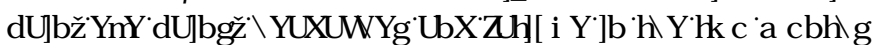

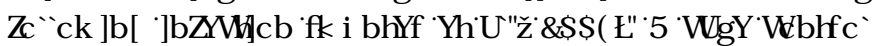

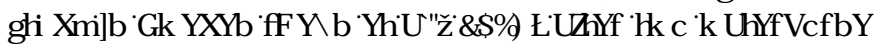
RXVZUHDMMRIC. hominis DRXQG WXDWFDMHZZ HHP RUHONHQ

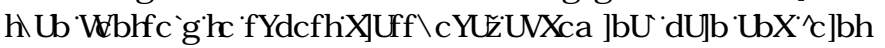

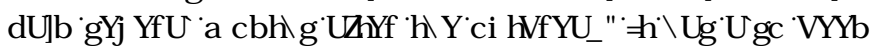
VXJ J HMHG [MKDWRCryptosporidium LQ HFWRQR D TFDXVH

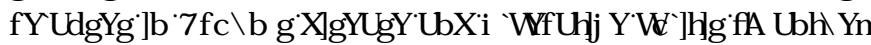

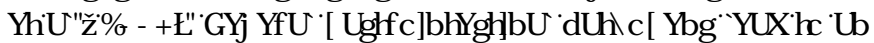

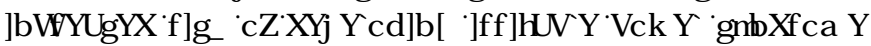

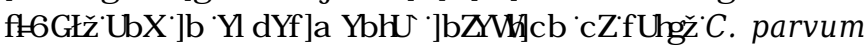

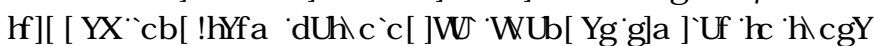

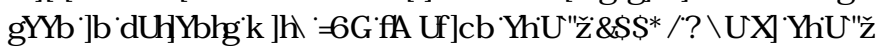
प्र०णा

\$WRFLDRQVEEHZ HQFFU SVRVSRUGLRVVDQGFDOFHUTKDYH

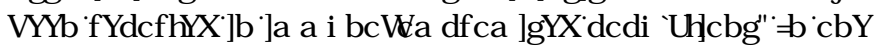

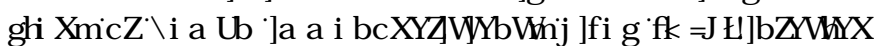

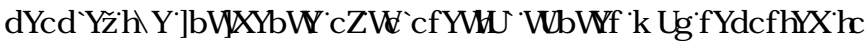




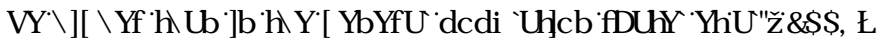
DQG[DLUXG IRI [SHRS

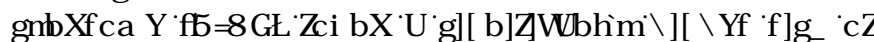
GHYHQRSLO] DPFRRQ FDUFLQRP D DPP RQJ [MKRVH ZZLVK

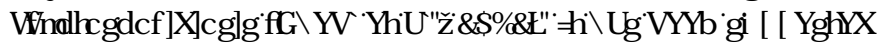

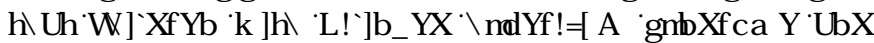

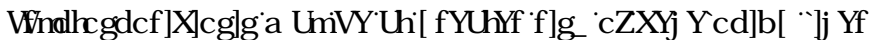

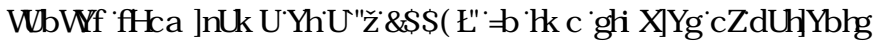

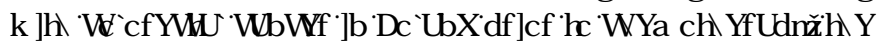

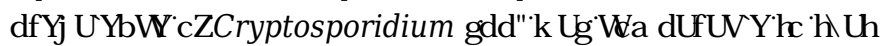

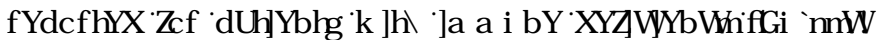

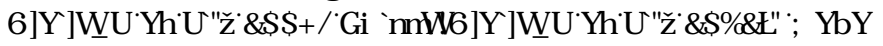
H SUHMRQTWXGLHMDQGDQGXFURQRI QRHRSQUMF[FKDO HMLQ

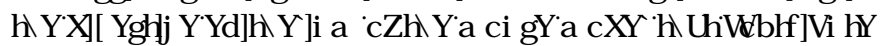

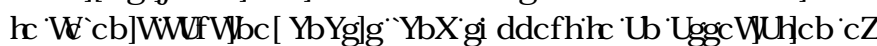

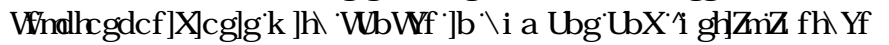

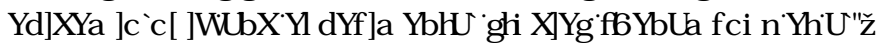
प्राप्या

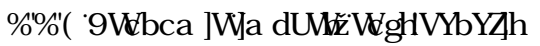

\$QKKRX] K[MKHUH[KDV[EHHQDDQRQFUHDVQ ]RFXVRRQ XQGHUWDQGL] [MKH [FQQLFDO[P SDFWRI IQI HFWRQRZLKK

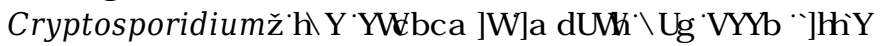
WXGHGWDQGIKHQFH[WKHUHDUH[QR[Cryptosporidium[VSHFULF

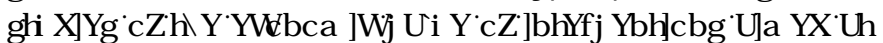

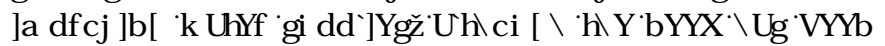
KL KQ] KUAG[ERVK DRU[GHYHCRSHGDDV[Z HQDVV[GHYHCRSLQ]

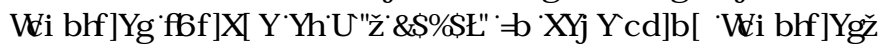

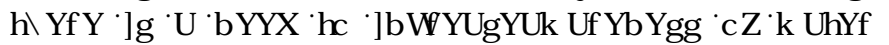

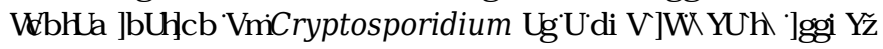
DQG[HYHQRQ[GHYHORSHG[FRXQMHMD UHDMAUFRQMGHDMRQ

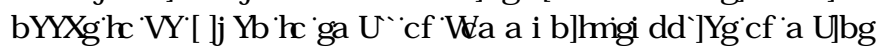
VXSSQHV [ZKHUHCCryptosporidium LV RQRWFRQMGHUG

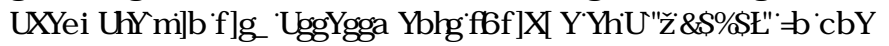

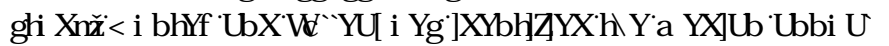
UWWRI GQI HFWRQZZ LVK CCryptosporidium IURP GYHU LYP DO

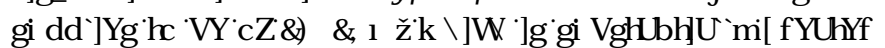

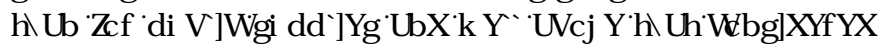

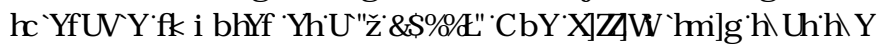

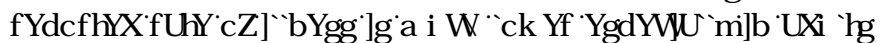

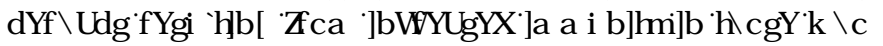

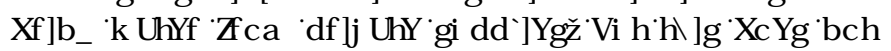
DFNQRZ OHG] H[MKH[UNVIMR I RXO] FFKLGUHQ]URP [MKHMH

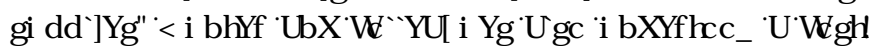

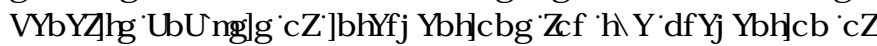

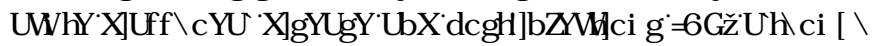

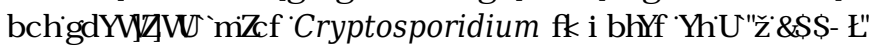

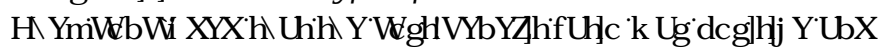

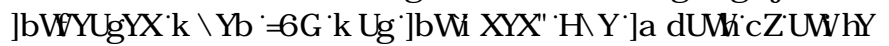

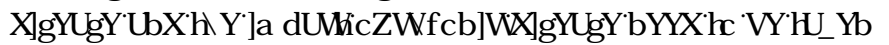

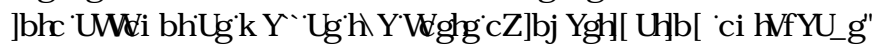

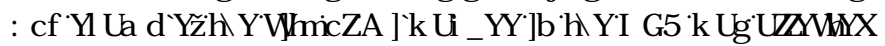
El IDCryptosporidium RXVZUHDNGQW

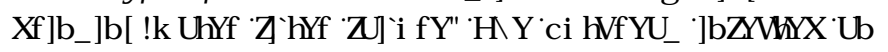

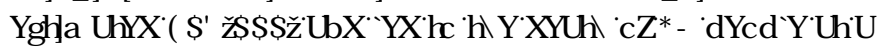

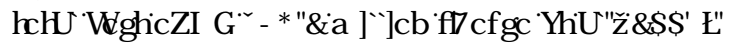

\subsection{Taxonomic Classification of the Agent(s)}

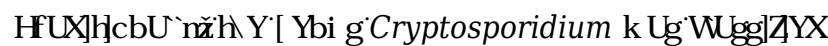

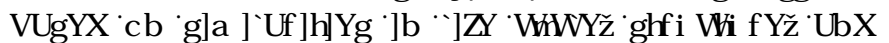

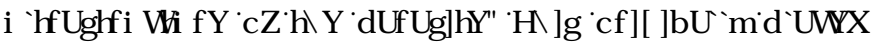
Cryptosporidium LQD] URXS[NQRZ QDDVMKHFRFFLGDLQTYKH

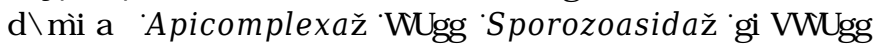

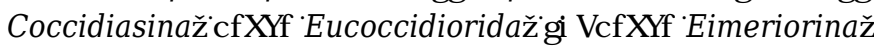

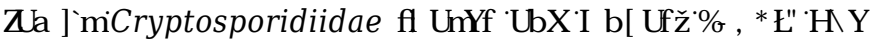

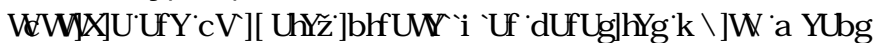

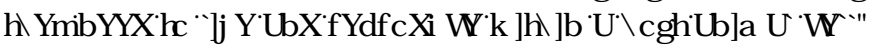

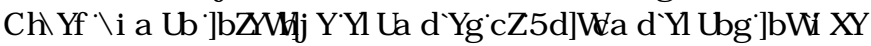
Toxoplasma gondii [DQGCyclospora cayetanensis $\mathbb{W H Z H Y H U}$ Cryptosporidium VDVKQHDMQRQTYKLVFFOMIIFDMRQDMP DQ

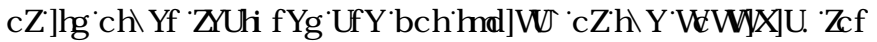

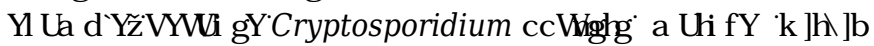

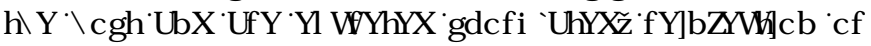

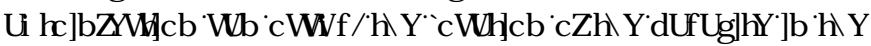

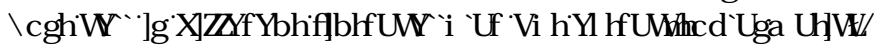
DQG][SHUKDSV $P$ RWIIP SRUDQVQTC ryptosporidium LV

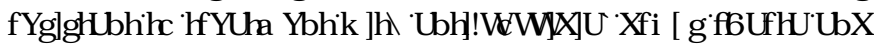

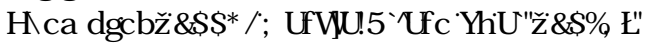

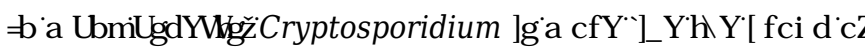
RU DQLVP VINQRZ Q TDVIMKH] UH DUQHVIQLKKHFCDW

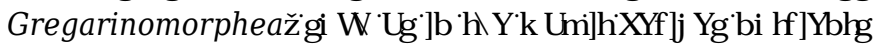

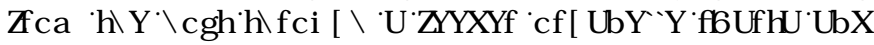

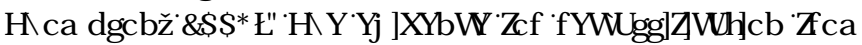
P RLSKRQP LFDQIELRQI IFDQDQGEELRFKHP IFDQGDUDIKDVEHHQ

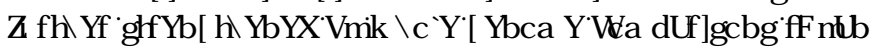
HMDQII]

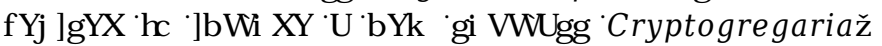

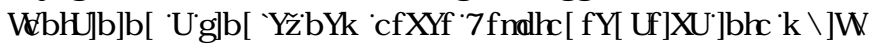
UKH $D D P$ LQ [Cryptosporidiidae KDVLQRZ [EHHQ[SCDFHG

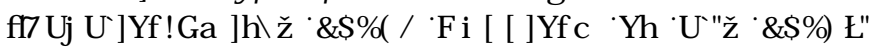
Cryptosporidium IVFXUHQV [VKHRQQ] HQXVLQTMKHDP LQ]

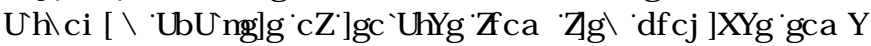
HYLGHFHDRUDTQHZ] HQXVPiscicryptosporidium IIEXVXXUKXH

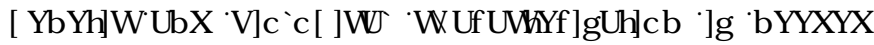

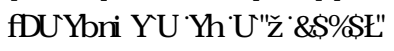

7KH]HFHQV FRQI LP HG DELLOW $\square$ RU[Cryptosporidium VR

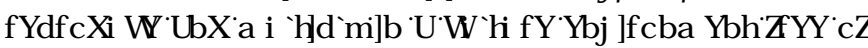

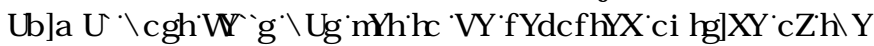

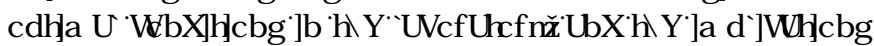

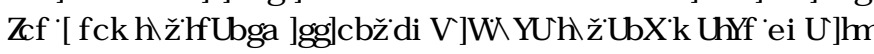

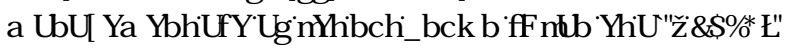

+LWRUFDQ DP DQ C Cryptosporidium VSHFLHEZ HUHQRDP HG EDMHGRQLVKHDDWXP SURQDMKDWVMKH ZZHUH[KRWWSHFU IF

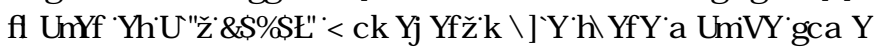
GH UHHRRI KRRWIDGDSWRQGRUP RWVWSHFLHMTWFWIKRWW

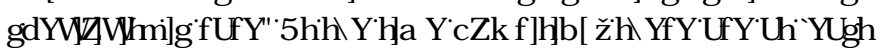

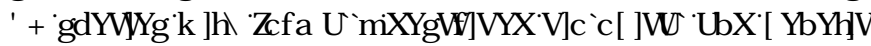

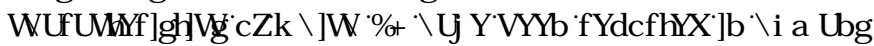

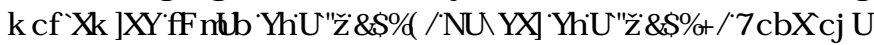
HMDQ

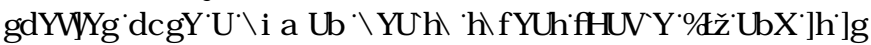

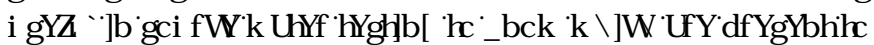
LQI RLP GUWDDMHMP HQWLCryptosporidium hominis DQG Cryptosporidium parvum DUHYKXHP DRRUKXP DQSSDMRRJ HQF VSHFLHV $R$ RI C Cryptosporidium DQG [KDYH \&ZRUGZ LGH

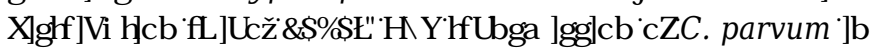

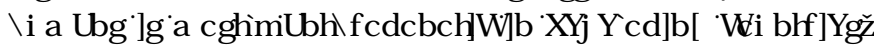

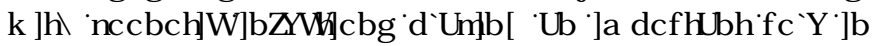

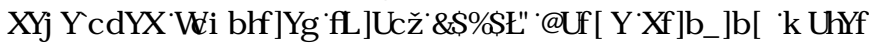


DURFDVAG FU SVRVSRUGLRVV IRXVEUHDNV IKDYH EEHHQ

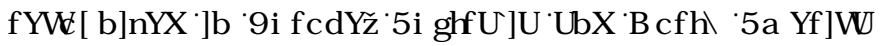

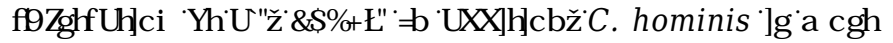

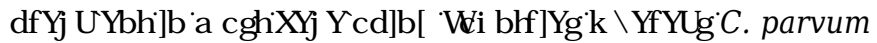

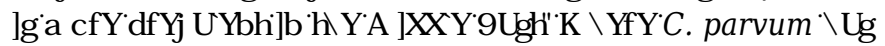

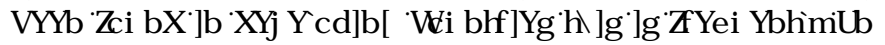

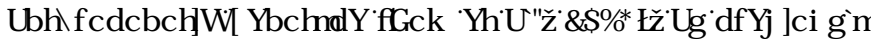

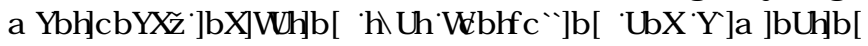
KXP DQTFRQMPP LQDMRQDDHIP SRUDDQNSLRMAFURQTP HDXUHN DLP HG [DW]UHGXFLQ] [MKH [SXEQF [KHDOK [ULVN [RI

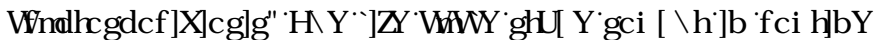

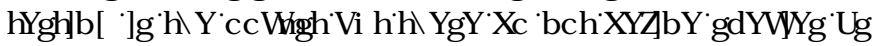

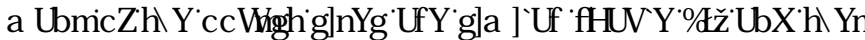

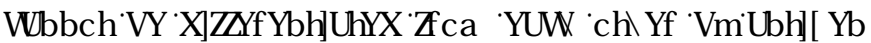
GHMAFURQDO ROFXOUP HMRRGVGHMFWD 01 \$ [M-TXHOFH

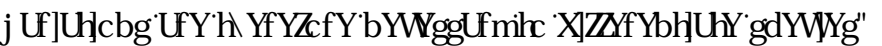
9DUDOW[GHMHFVHG [E] [MHTXHQFLO] [MKHLYP DQOUXEXQLW

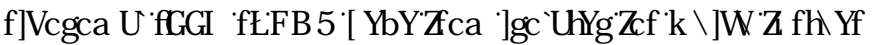

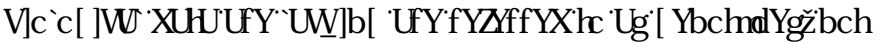

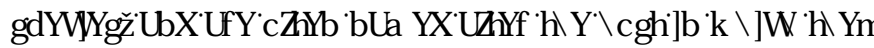

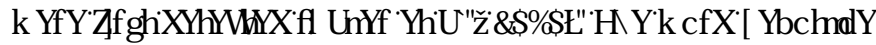

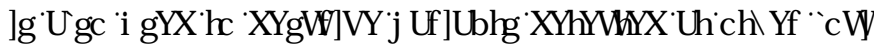
ZLMLQGGLOHEYSHFHM

Table 1. Formally described Cryptosporidium species, their oocyst sizes, major hosts and reported human infections

\begin{tabular}{|c|c|c|c|}
\hline Cryptosporidium species & $\begin{array}{l}\text { Mean Oocyst } \\
\text { Dimensions } \\
(\mu \mathrm{m})^{\mathrm{a}}\end{array}$ & Major Host(s) & Reported Human Infection \\
\hline C. andersoni & பயாயா & $\& D W O A$ & ४HШEXVQDUH \\
\hline C. avium[ISUHYRXVQDYLQ] HQRWSH9] & பயாயா & \%GV & $1 \mathrm{R}$ \\
\hline C. apodemi & 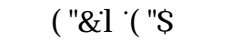 & 5RGHQW & $1 \mathrm{R}$ \\
\hline C. baileyi & பாயா & $3 R \times O 4$ & $1 \mathrm{R}$ \\
\hline 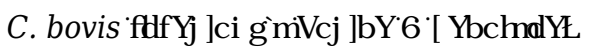 & பயாயா & \&DWOH & $\varangle \mathrm{M} \mathbb{E} \times \mathbf{Q D O H}$ \\
\hline 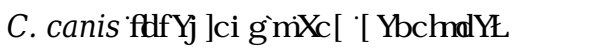 & பயாயா & ' R & $\triangleleft \mathrm{H} \mathbb{R}$ RFFDMRQDO \\
\hline C. cuniculus [SUHYIRXVQ[UEELV] HQRWSH] & பயாயா & 5DEELVIVXXP DQV & $\begin{array}{l}\text { HMIRFFDMRQDQR QHGUQNG } \\
\text { ZDMAURXVIUDNUHSRUAG }\end{array}$ \\
\hline C. ditrichi & ப⿴囗十 & 5RGQW & $1 \mathrm{R}$ \\
\hline C. ducismarci & 15 & 7RURLLHN & $1 \mathrm{R}$ \\
\hline C. erinacei & பயாயா & +HG HKR & $\varangle$ MIEXVQDUH \\
\hline $\begin{array}{l}\text { C. fayeri } \text { [SUMLRXVQP DUXSLD HQRWSH } \\
\text {, }\end{array}$ & பயாயா & 0 DUXSLDO & $\triangle \mathrm{MUE}$ EXVOUH \\
\hline C. felis & பயாயா & $\& D W$ & $\varangle H \mathbb{R}$ RFFDMRQDO \\
\hline C. fragile & பயாயா & \%DFNVSLQHGMRDG & $1 \mathrm{R}$ \\
\hline C. galli & பயாயாய & \&KIFNHQ & $1 \mathrm{R}$ \\
\hline C. homai & 1 RVREVHUYG & * XLQHDSL & $1 \mathrm{R}$ \\
\hline $\begin{array}{l}\text { C. hominis [SUHYRXVQC. parvum[KXP DQ } \\
\text { J HQRWSH四 HQRWSH四DQ] HQRWSH[+ }\end{array}$ & பயாயா & $+X P$ DQV & 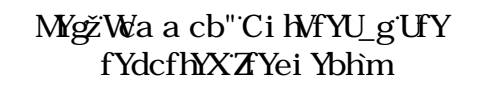 \\
\hline C. huwi[SUHYRXV[piscine J HORWSHய] & பயாயா & * XSSI & $1 \mathrm{R}$ \\
\hline $\begin{array}{l}\text { C. macropodum ШSUMLRXVQP DUXSLO } \\
\text { J HQRWSH, }\end{array}$ & பாயாய & ( DMALQ] UH [NDQ DRR & $1 \mathrm{R}$ \\
\hline C. meleagridis & பயாயா & \%UGM DP P DQ & 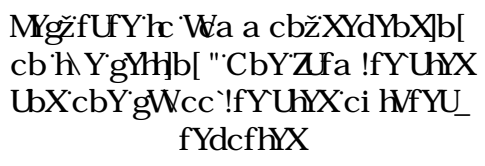 \\
\hline C. molnari & பயாயா & 6HDEUHDP & $1 \mathrm{R}$ \\
\hline C. muris & பயாயா & 5RGQW & ४HMEXVRQQWDUHQ \\
\hline C. occultus & 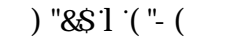 & 5RGHQW & $1 \mathrm{R}$ \\
\hline 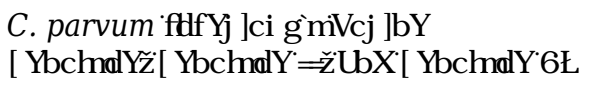 & பயாயா & $\begin{array}{l}\text { +XP DQVIISUHZHDQHG } \\
\text { P DP P DODOOOYHMRFN }\end{array}$ & $\begin{array}{l}\text { ४HMFRP P RQIR XVEUDMMDUH } \\
\text { UHSRUAGIUTTXHQNQ }\end{array}$ \\
\hline C. proliferans & ப⿴囗十丁 & 5RGHQW & $1 \mathrm{R}$ \\
\hline C. ryanae [SUHYRXVQ GHUONH] HQRWSH] & பயாயா & \&DWOH & $1 \mathrm{R}$ \\
\hline C. rubeyi & ப⿴囗十丁口卄 & * URXQGVTXWHQ & $1 \mathrm{R}$ \\
\hline C. scrofarum & பயாயா & 3! & ४HMEXVUDUH \\
\hline C. serpentis & பயாயா & 5HSWOAN & $1 \mathrm{R}$ \\
\hline
\end{tabular}




\begin{tabular}{|c|c|c|c|}
\hline Cryptosporidium species & $\begin{array}{c}\text { Mean Oocyst } \\
\text { Dimensions } \\
(\mu \mathrm{m})^{\mathrm{a}}\end{array}$ & Major Host(s) & Reported Human Infection \\
\hline 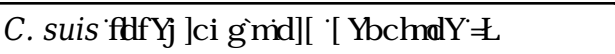 & பயாயா & 34 & $\varangle \mathrm{M} \mathbb{E} \times \mathrm{QWDH}$ \\
\hline 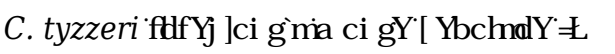 & பாயாய & $0 \mathrm{FFH}$ & $\varangle$ MIEXVQDUH \\
\hline 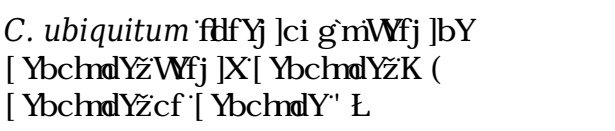 & பயாயா & 9DURXVP DP P DQ & $\triangleleft \mathrm{M} \mathbb{R} R F F D M R Q D O$ \\
\hline C. viatorum & பாயாய & $+X P$ DQV & $\varangle \mathrm{M} \mathbb{R}$ RFDMRQDO \\
\hline $\begin{array}{l}\text { C. varanii } \\
\text { पM Q } \mathbb{C} \text {. saurophilum } \square\end{array}$ & பயாயா & 5HSUON & $1 \mathrm{R}$ \\
\hline C. wrairi & பாயாய & * XLQHDSL & $1 \mathrm{R}$ \\
\hline 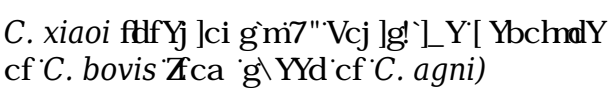 & பயாயா & 6KHSس RDW & $1 \mathrm{R}$ \\
\hline
\end{tabular}

\section{5 四 RVD HSRUMAG}

\begin{abstract}
' UIHUHQVWNSHVRI P ROHFXOUGLI QRWF[YRRQVKDYHEHHQ

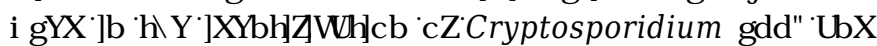

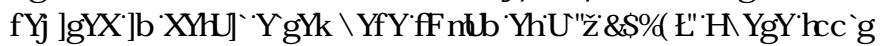

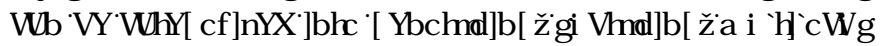
WSL] [SRSXCMRQ] HQHMFVIDQG[FRP SDUMYH] HQRP IFV GHSHQGL [RQLYKHDSSURDFKHMDQGXXD] HMTKHMH[L KQ GLVUP LQDURU IP RGIFXDULMFKQLTXHNDDORZ IONDUSHFUL GIHUHQWDURQRI [C. parvum DQGC. hominis ZZKIFK DDOVYR VKHLGHQW LFDMRQRI PP DQ [VXEWSH]DP LOHNDDQGP DQ VXEWSHV [Z LVKLQ GHDFK IVSHFUHV [6XEW SLQ] [RI Cryptosporidium VSHFLMTSHFLFDQ [C. hominis DDQGC. parvum TFDQ[SURYLGHFOUW RI IP RGHRI [WDQVP LWRQRQ DGGLURQ [VR [EHLQ] IP SRUVDQWHSLGHP IRQP IFDOCMRQQ

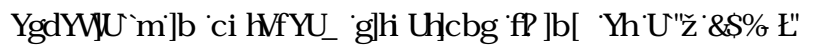

\subsection{Transmission}

3HRSOFEHFRP HLQ HFUAZ LLEC ryptosporidium HUMUEI

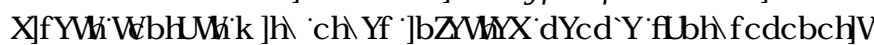

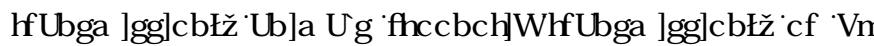
LQ HMRQRI FRQMDP LQDMAG]RRGRULZ DVAU IIRRGERQHRU

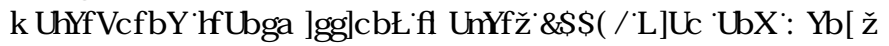

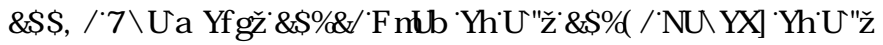

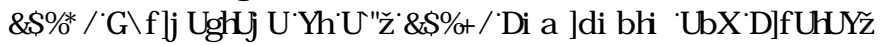

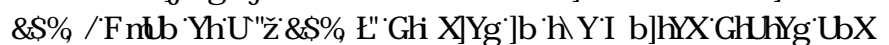

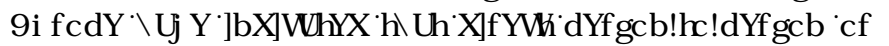

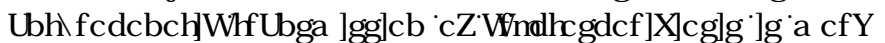
FRP P RQW LRس ИKHIDFTXLVWRQRI FU SVRVSRUGIRVVIQRKXP DQVIKDVEHHQ

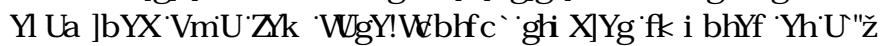

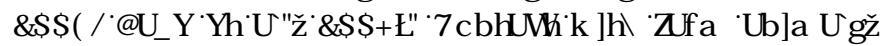

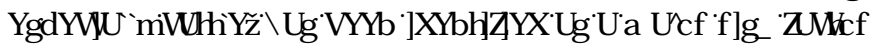
IRUISRUDGF [FU SURVSRUGRVVDQRQGXWUDO] HG[FRXQWHN

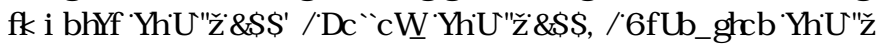
प्व०ण

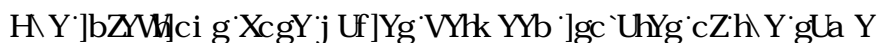

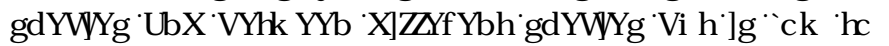
UHDVYHQ LRZ IRUTERVK CC. parvum DQG CC. hominis

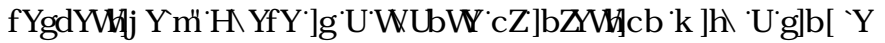

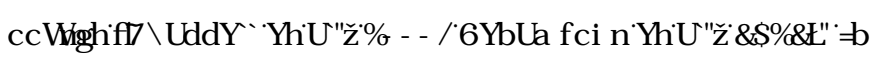
KXP DQTYRQQMAHUWXGLMDP HGDQLQ HFWRXVGRVHTRUC.

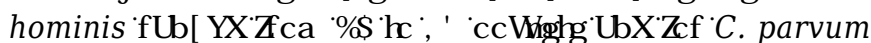

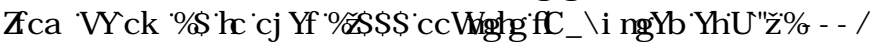

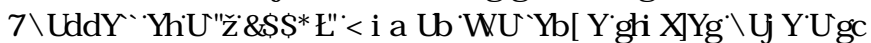

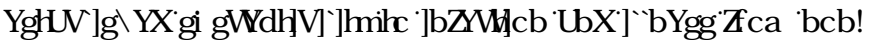

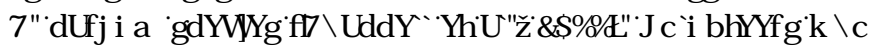

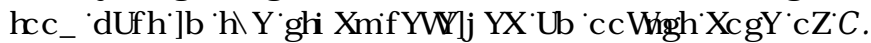
meleagridis [MKDWZRXG RQNHQ [EH

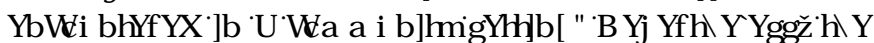

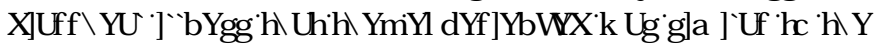
LOHWLHSRUAGLQRDXXDQ DFTXLHGEC. meleagridis

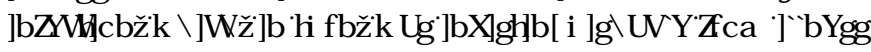
FDXVGEI CC. parvum RUC. hominis $\square$

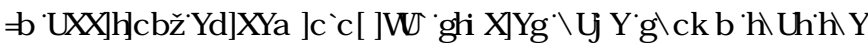
J HRJ USSKLFDQGLWEEXURQVRI [Cryptosporidium VSSDYDU

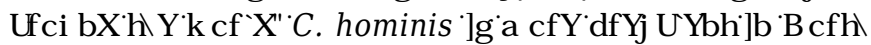

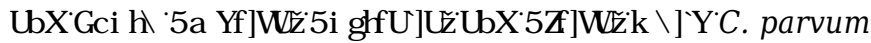

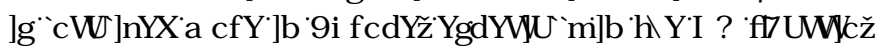
प्राप्या

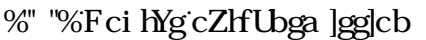

Cryptosporidium P DQQQ [Q HFW[MKH] DWRLQMAMQDO

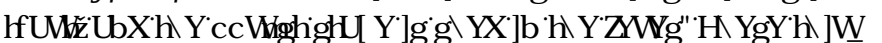

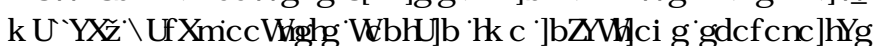

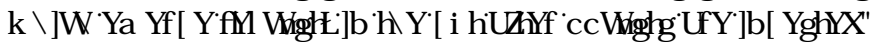
7KH[VSRLR] RLVANDQI HFVIMKH[FHQQ/QQLQ] [VKH] XVIEHRUH

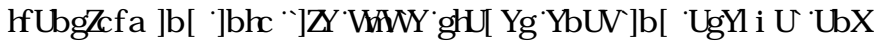

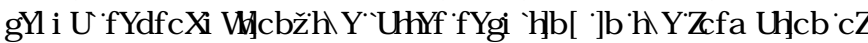

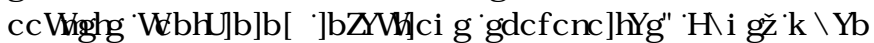

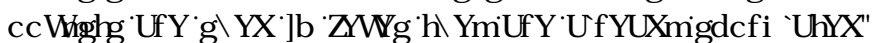

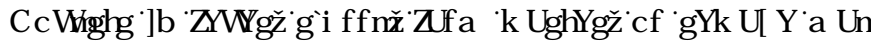

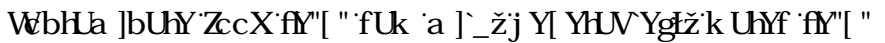

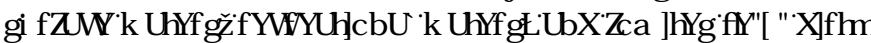

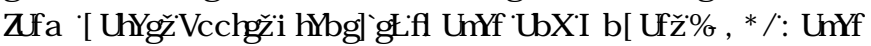

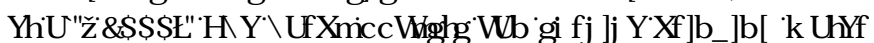

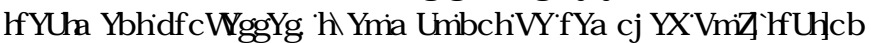

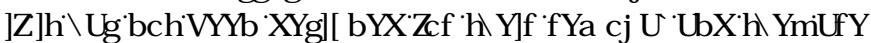

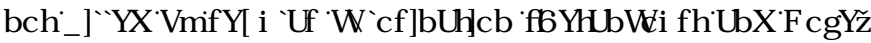




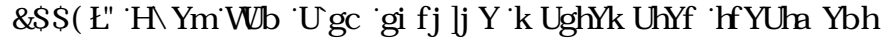

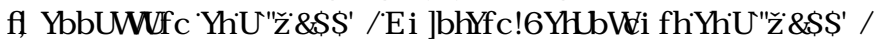

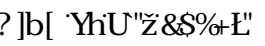

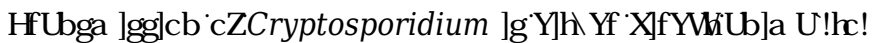

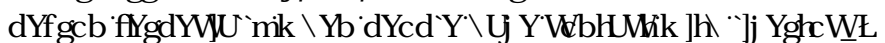

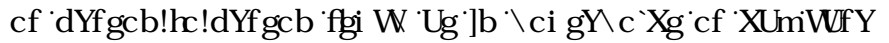

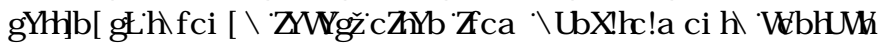

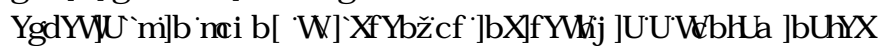

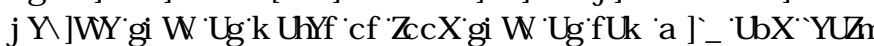

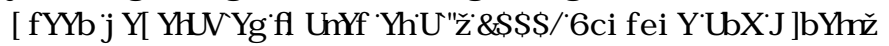

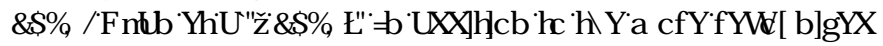

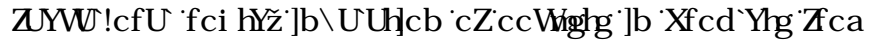

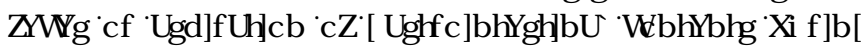
YRP LVQJ PP D FDXVH IUHVLLVRU FU SVRVSRUGLRVV

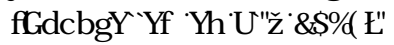

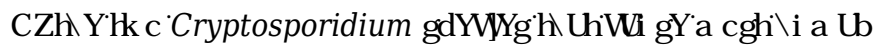
FDVHVRI TFU SVRVSRUGIRVVIC. hominis LVIWDQVP LMAG EHVZ HHQ[SHRS OHIDDQMXLRSQRWF]ZZKHUDVEC. parvum LV

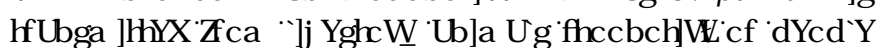

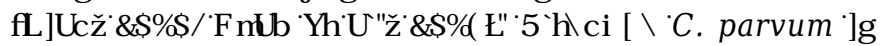
VRP HMP HVIRXQG[QFRP SDQLRQDQPP DOTTMKH ] HQHDQ

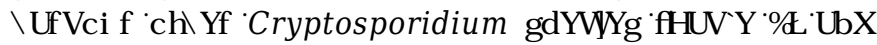

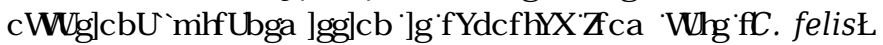

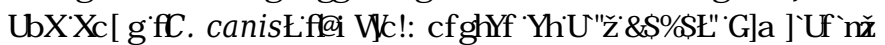
Z LCSOHIKDUERXUDTUDO HRI [Cryptosporidium VSHFLHMDQG

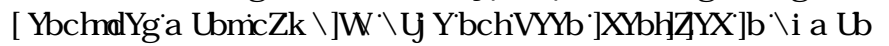

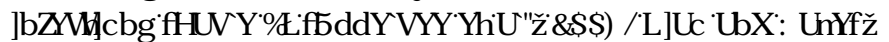

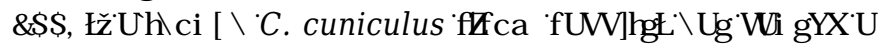

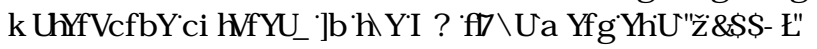

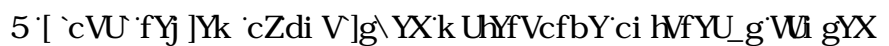

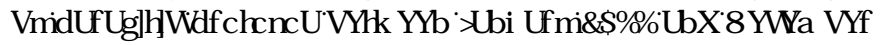

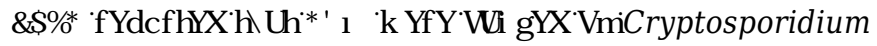

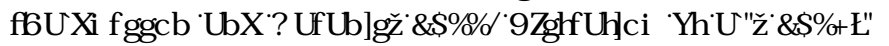

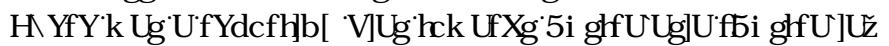

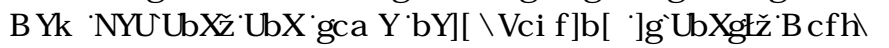
\$P HUFDLDQG XURSHLZ KHUHIXXUYHLODQFHIM WAP VIDUH HMDEQVHG]\$ QKRXJ K P RWVZ DUALERLQH RXVZUHDWWDUH FDXVHGEE $[C$. parvum RUCC. hominis

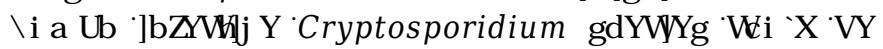
WDQVP LWHG [MKLRX] K [GUQNLQ] [ZDVHU] IWEHFRP HN

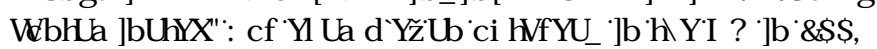

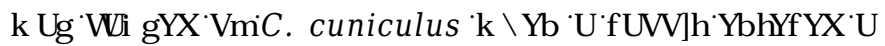

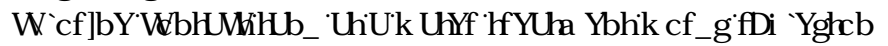

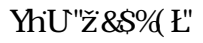

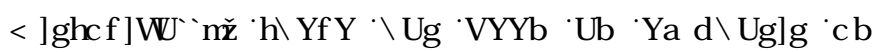
Cryptosporidium DYDEZ DUAERLQHSDMKRJ HQ ISH KDSVYMRUMH

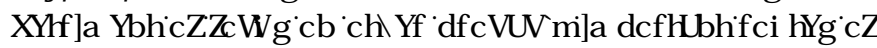
WDQVP LMRQ][SDUMFXOUQ TRRG I K KVAHQ RXVEUHDN

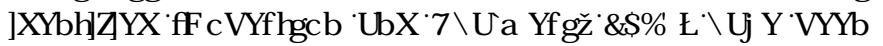

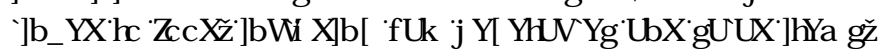
KHEVTDZ P HDMGXQSDMAXUMHGP LONDQG]GDU [SLRGXFW

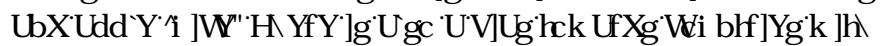

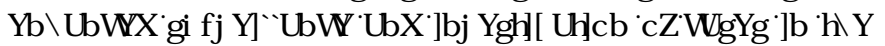

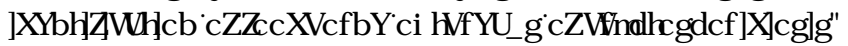

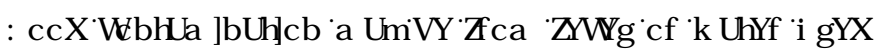
GXUQ] TRRG[SURGXFURQ][SURFHMQ] IRU[SUHSDUDRQ]

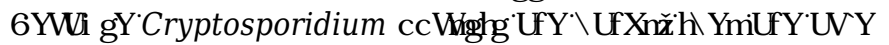

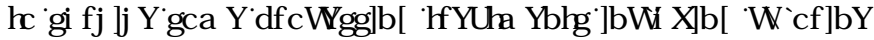

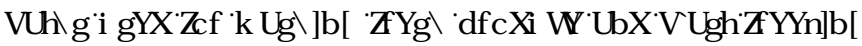

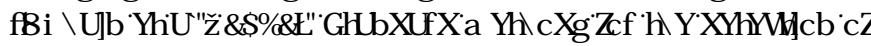
Cryptosporidium LQDQ IRRGWXII Z HUHRQQ [SXEQWHGRQ

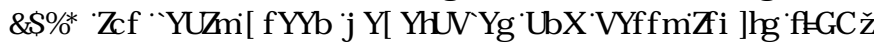

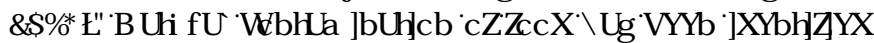

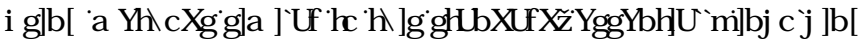

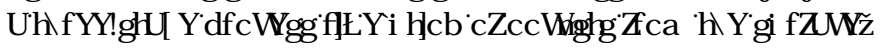
QU⿴囗FRQFHQVDWRQ DQG [UHP RYDO] URP [GHEUV [EI

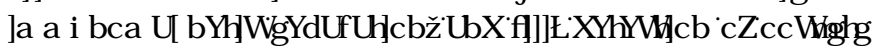
EI IP P XQRIQRLUMFHQFHPP IFURVFRSI四) \$四

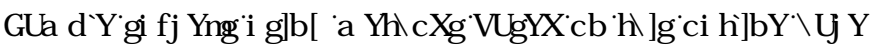
UHSRUAG C Cryptosporidium RRF। WW $\square$ Q [SURGXFH $\square$ URP

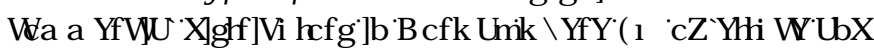

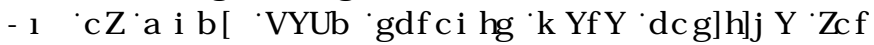
Cryptosporidium [5 REHUNRQDDQG [* NHUGH

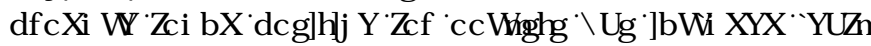
J UHOM

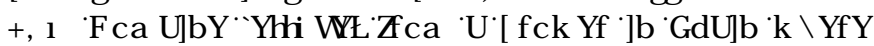

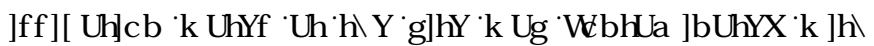

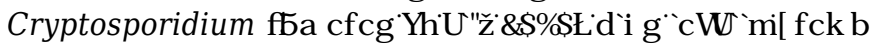
SLRGXFH]LRP QDUP HUVP DUNHMLQTBRDQG[Z KHUHRQH[QHHN VDP SOIRROFFHOUU [MDP SOIDOG]RXUFDEED HLIP SOMZHUH

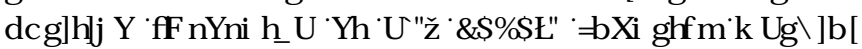

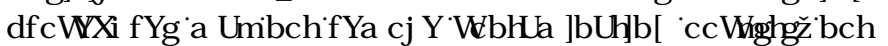

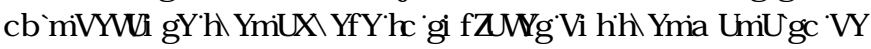

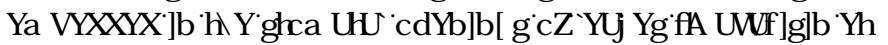

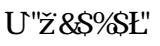

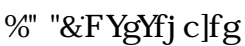

Cryptosporidium VSSTR HFVDRZ LGHDDOJ HRRI DDQP DO KRWWRI GGUIHUHQWFGOWHMIEXVIP DQQUHMHYRLUMRI KXXP DQ]

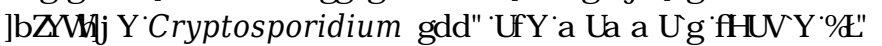
$\$$ QRKRXJ K[C. hominis [DQGRQHRI LMKHDQMLLRSRQRMF[WXEWSHV

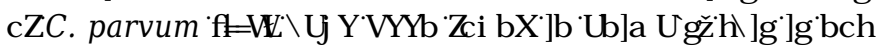

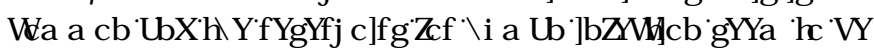

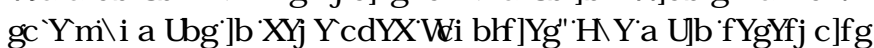
RI]RRQRWF[C. parvum [DUHD RXQ [UXP LQDQWWFDWOIIKHHS]

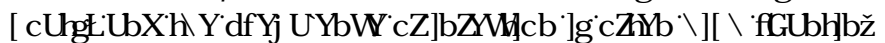

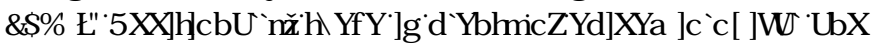

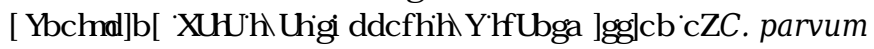

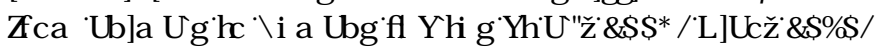
5। DQRHMDQM

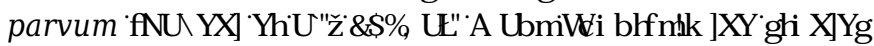

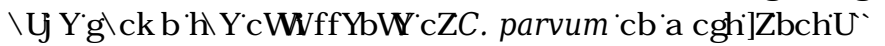
IDP VZKHUP DQ DQIP DQZZLMLQTHKHIKHURUII QFFEEHFRP H

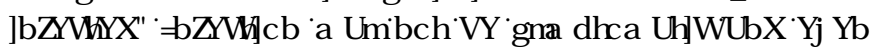

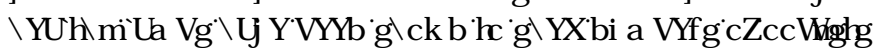

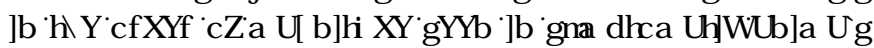

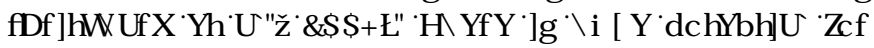

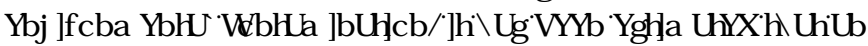

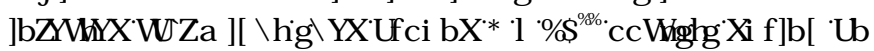

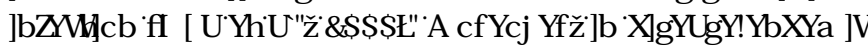

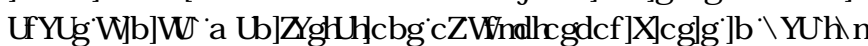

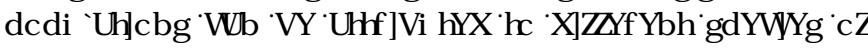
Cryptosporidium $\square \square \square$.

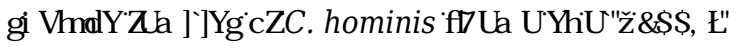

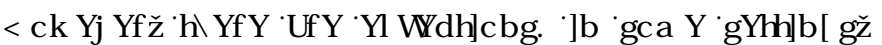

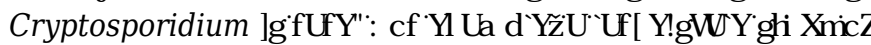




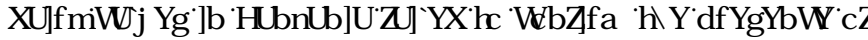

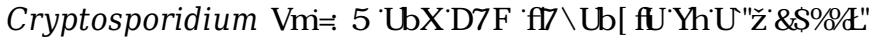

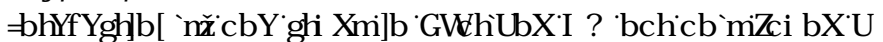

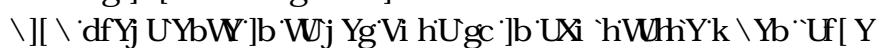

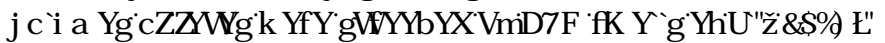
, WIVVIKHUHI RUHПP SRUDQWVIKDWUUVNDDMHMP HQWDDUH VXSSRUHG [E\ [RFDOTQI RUP DVRQT] Q DGGLVRQ][SRLQW SUHYDOLFHDDQG[RG LXGQDQMXGHMKDYH[GFXP HQMGGD H UHOUAGSDMALONRICCryptosporidium VSHFLHROGGDU FFDWOH

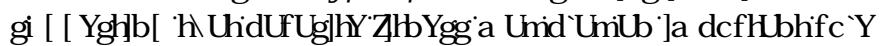
LQTMDOMP LMRQRI FU SURVSRUGLRVVDP RQ [FDWOHDQGIR

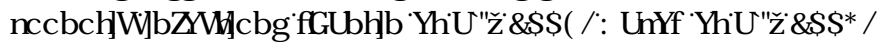
; LRR

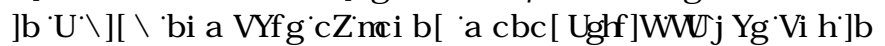

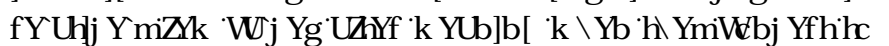
UXP HQDQQXWURQWI) D HUFMMDQm]

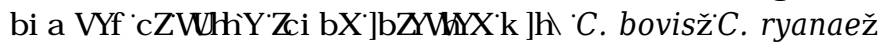

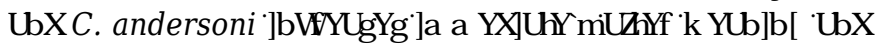

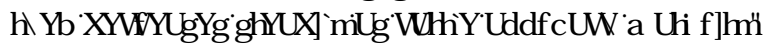

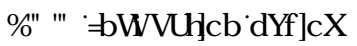

7KH[KXP DQROFXEDMRQ[SHURG[EHVI HHQRO] HMRQRRI

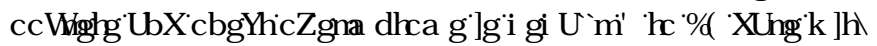

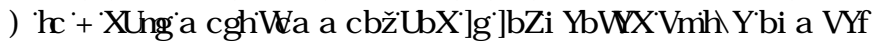
RI RRFI WILQ HMAG IKRWMP P XQHUHNSRQMHMIDQGRMXHUKRWW

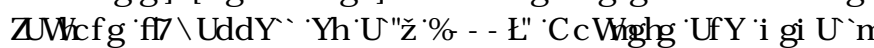

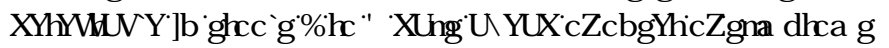
[\&KDO HUVDQG DYHMM

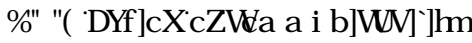

,QI HFWRXVIVSRLXOUMAG [Cryptosporidium RRFI WW DUH

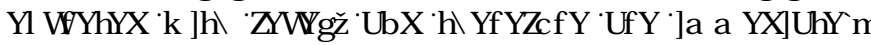

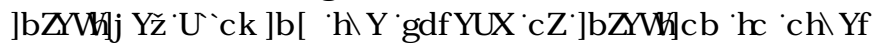

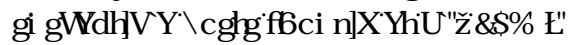

7KHCX P SURP VRI DFXXUFU SURVSRUGRVYFDQEHSLRRQ HG

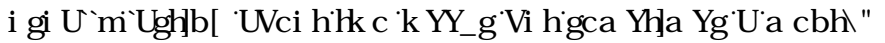

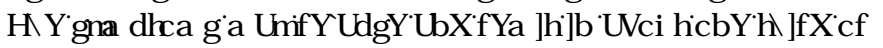
P RUHRIFDMHMD DF. HQ] HTHMDQ]

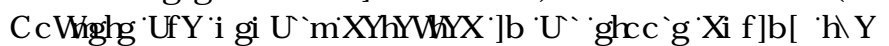

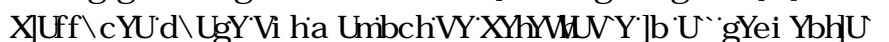
WRRQ/GXUQ] [UHFXSHUDRQRULDM P SVRP DMF GQIHFURQ ¿\&KDSSHOHMDQM]

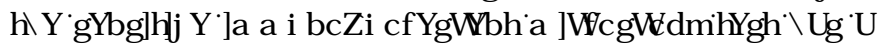

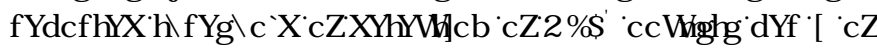

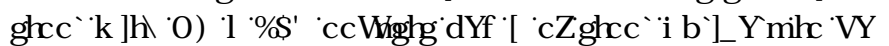

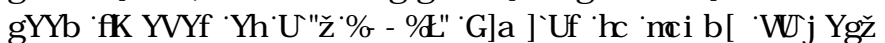
V P SURP DUF [FKLQUHQTKHGIKL K RXP EHUVR RRFI WWMQD

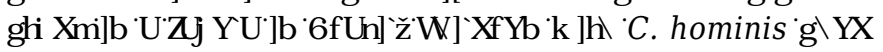

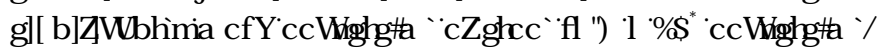
UDg Hயm

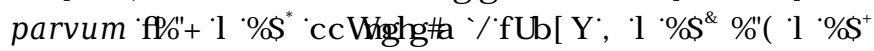

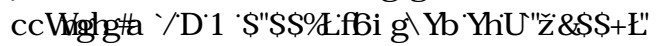

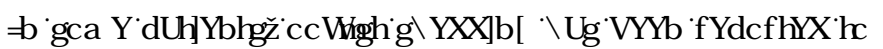

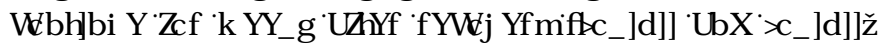

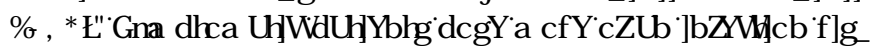

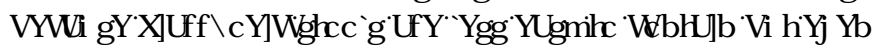
DM P SVRP DUF [MKHGGL] [P D FFXVHFFRQVP LQDMRQTRU H[ DP SOH[MKLRX] K[VZ LP P LQ] [SRRQDDQGZLOFRQMEXVA[I/R
RRF WWLQTKXP DQIZDMAIDQGZZ DWAZ DUA UC ryptosporidium RRFI WWDUHIKDUG LDQGJHMWDQVIMRP DQ IHOYLRQP HQVDO SUHMXUHMLVXUYLYDQIV [OU HQ [GHSHQGHGRRP RLWXUH

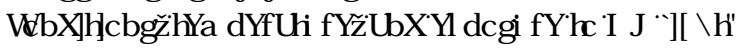

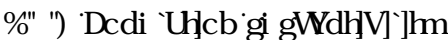

Cryptosporidium LVUHFRJ QL]HGDVDD[ODGLQ [FDXVH[RI

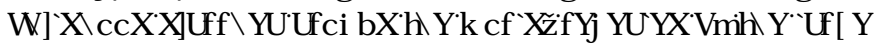

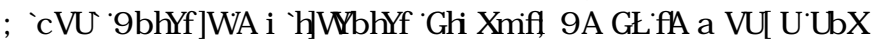

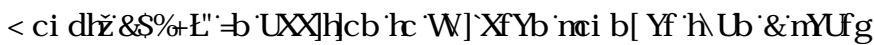
RQELCryptosporidium LV[SDUMFXOUQ [SLRE@P DMF TQDMKH

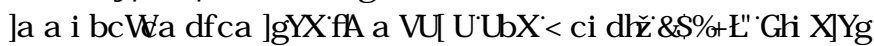

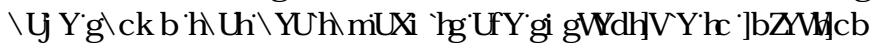
ZLIKLYP DOSXP EHURI Cryptosporidium RRF WWMHXQR

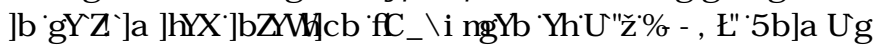

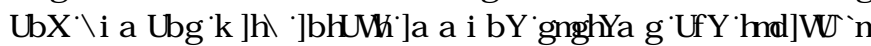

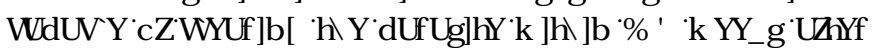

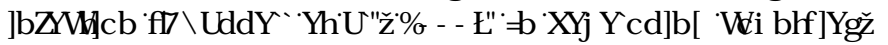

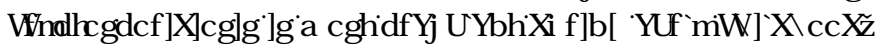

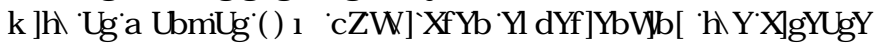

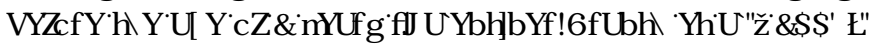

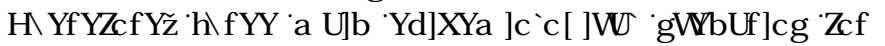
Cryptosporidium KDYHEHHQGHFUEHQIIIIUSRLGGIFIRI UAQ Z DUAUUHCOUHG]RXVEUHDNVRI [MHO[OP LWO] [GDUKHDLQ

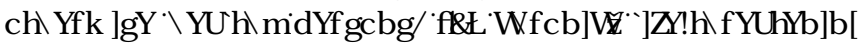

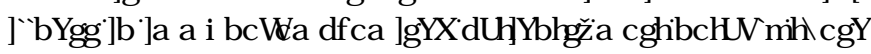

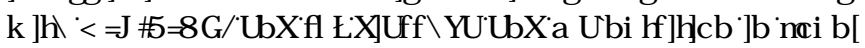

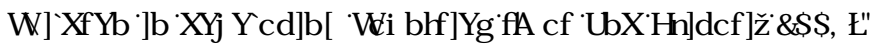
7KH[KL KHWVEXUGHQRI [Cryptosporidium LQ HFWRQZVGQ

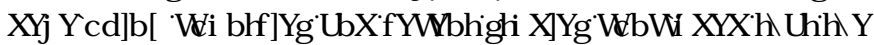

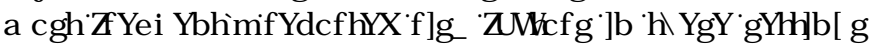

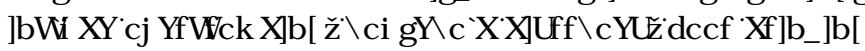

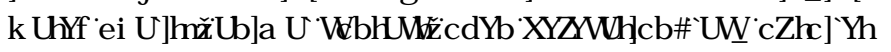

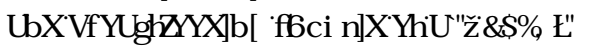

\subsection{Population and Individual Control Measures}

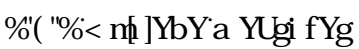

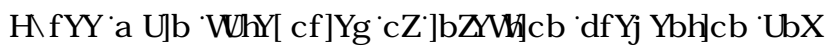

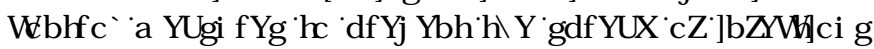

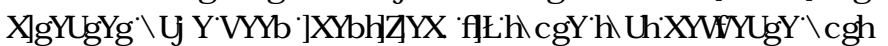

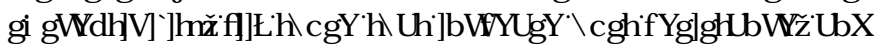

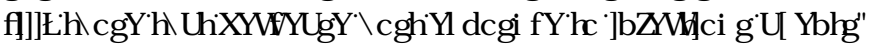

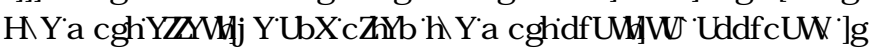

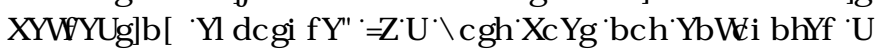

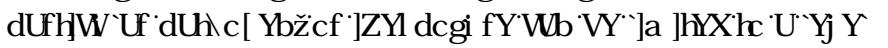

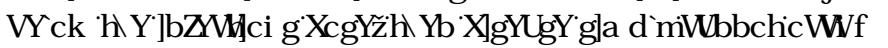
[\$QGHURQW]

Cryptosporidium IVKL KQTFRQVI IRXVDQGFDQEF ZDMAUIRRG IIRLQIIKDQGYDQGP DMAUDQRUVYXUDFHMUKXDMKDYH

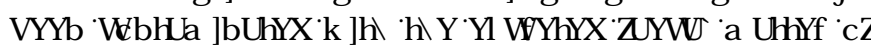

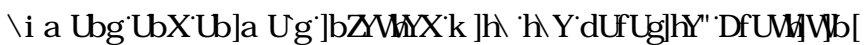

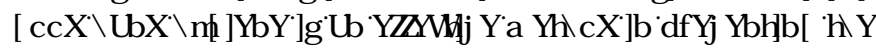

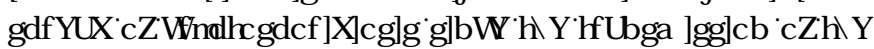

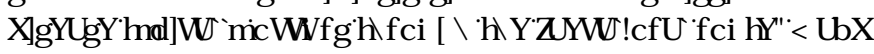

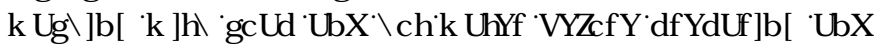
HDMQ] QRRGIDIVAU[GH HFDMRQRUTFKD] LQ] [GLSHUVDQG

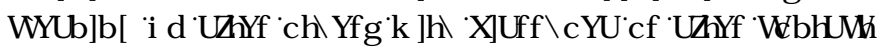
ZLLKGRP HMFERUIDUP DQPP DQIIVMKHLMP SOMMIP RWLEDMF 


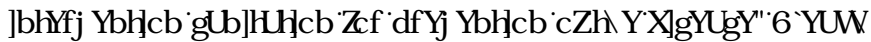

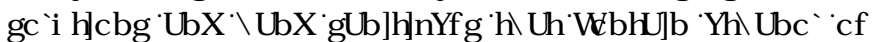
LVRSLRSDQRODVIVKHLDFUYHP IFURELFLGHDUH[QRVYHIIFUYH

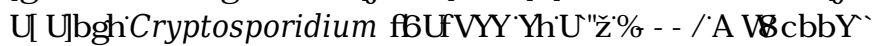

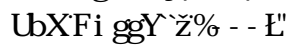

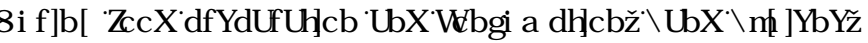

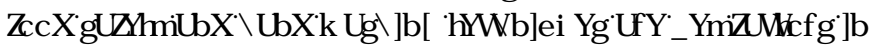
SUHYHQMQ] [MKHLVSUHDGRII FU SVRVSRUGLRVVIDQG RUKHU HQVAUF [GLYHDVHM[] DVKLO] RRU[SHHOQ] [UDZ IUXLWDQG YHJ HVDEON [MKRLRX] KQ [EH RUH [HDWO] [DQG DYRLGLO] XQSDMAXUUHGP LOVDQG]UXLVMXIFHVIFRQMUXXVHIPP SRUDDWW P HDVXUHVRQ[MKH[SUHYHQWRQRI FUU SVRVSRUGIRVVD RRG KDQGQQ] [HP SQR HHN[H SHUHQFL] [GLUKRHDRRURVKKU

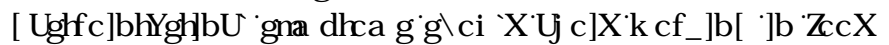

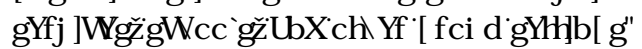

,QTMA-DUAG]UFUHDURQDQZZ DUAUYYHQXHNTVXFK [DVNZ IP P LQ

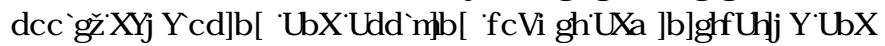
HOJ LQHHUQ] FFRQRRQ/DUH[P SHUDUYH[MR_UHGXFL] [MKH

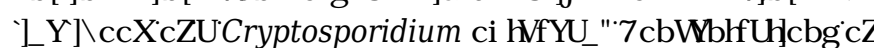

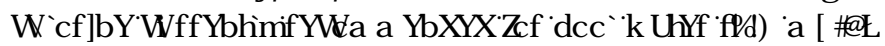
GR[QRVMQDFUYDMLC ryptosporidium RRF WMLQDTWP HUDP H

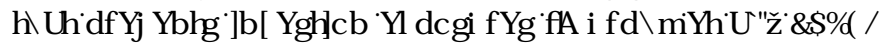

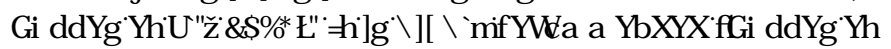

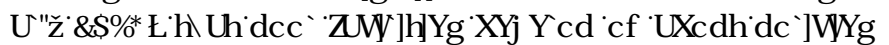
H FOGL, [OH XDUMDQGIVZ IP P HUH SHUHQFL [GDUKHD

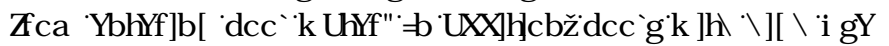

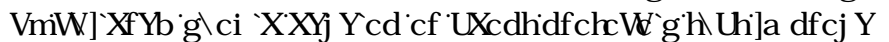

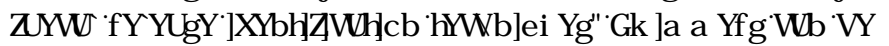
H SRVHGLYR \&U SVRVSRUGIXP [RRF WWEH RUFFRQMIPP LQDMAG

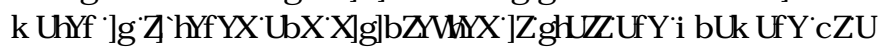

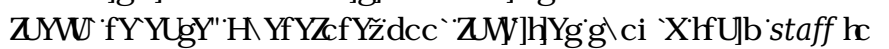
UHFRJ QL] HDDHFDQUHOHDVHNDDQG]HGXFDMA[SDWRQVIRQ[MKH IP SRUDOFHRIUHSRUMG IHODMHI

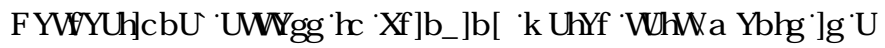

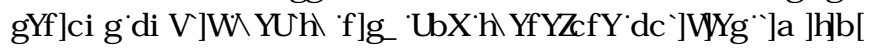

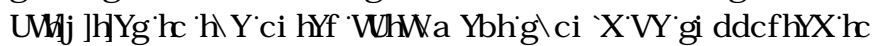
DYRLG GFRQVDP LQDWRQ RRI [GULNLQ] [Z DVHU LXSSOHV

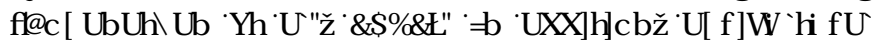

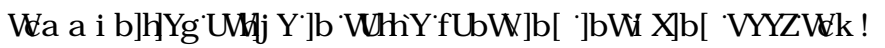
FDORSHOWRQVIZKHHDQIP DQYMAQG[VR[EHPP RUTFRQ LQHG] UHSUHVHQWDD [NHULRXV [SXEQF [KHDOKK [FRQFHUQ]

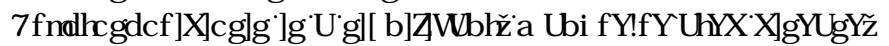

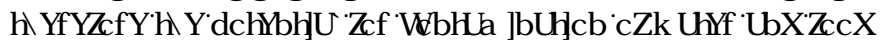

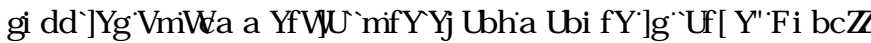

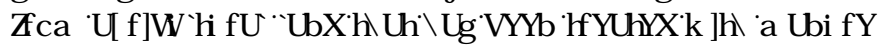
KDVMMKH[SRUAQWDQURLFRQMIPP LQDUA[RRFDQVXU DFHZZ DUALDQG Z HQ/[MKDVVIXSSQ [ZDMAU]RULKXP DQ[FRQVXP SWRQT/KH UHMRXLFHP DQD HP HQMRR [MKHDD UFXQX LDQZ DWALWMHDP IVV

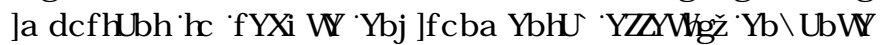

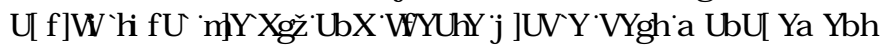

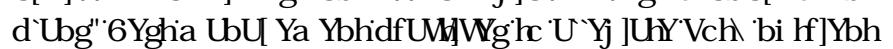

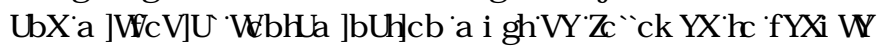
SXEQF [KHDOK [HII IFWTD RUTOWDQFHDUHMGXDO/QURP D) UFXQXUDQDFVULLHNIP XWVEH[SLRFHMHGLDGHTXDMAQ [YR

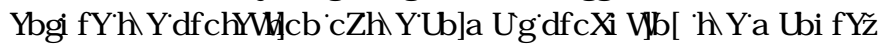

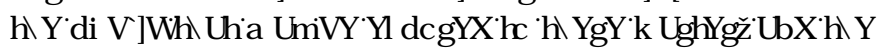
HQYLLRQP HQVQ

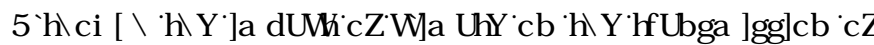

GDUKHDQSDMKRJ HQV]UP DLVIXQFHUDLQTMHYHDOUXGLH

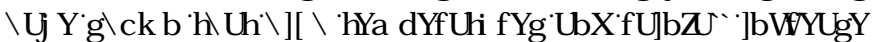

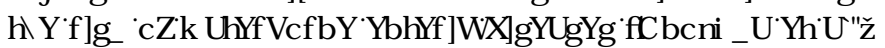

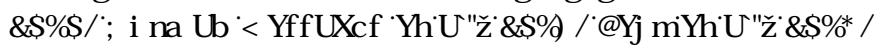

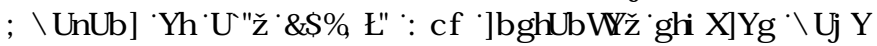
GHP RQWWDHA LIKDWFRQNDP LQDMRQRII GGUQNLO] [ZDVAU

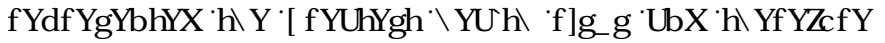
LQVAUYHQMRQV [GHM] QHG [VR GQFUHDVH[GUQNLQ] [ZDVAU WHDVP HQWZZ HUH[QHFHMDU IRQRZLQ] [SHURGVIRI [KHDY

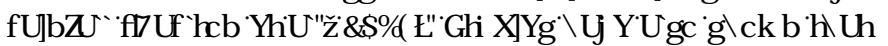

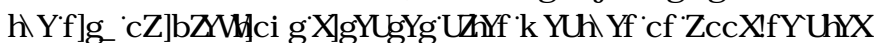
QDUXLDQGLVDMAUVDOKRXJ K[VSHFU F[YR_LKKH[YYHQVMWHOTIV KL KQ [GHSHQGHOVRQDDRXX EHURI DDFVRUVD KHMHDDFVRUV

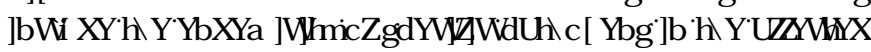

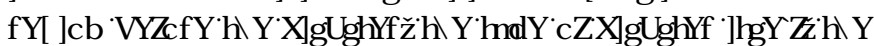

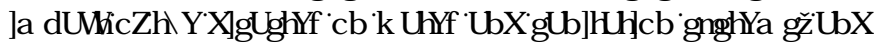

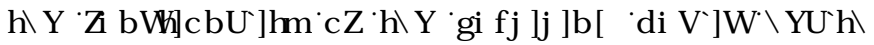

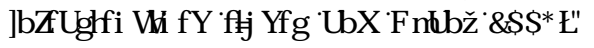

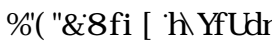

\&U SURVSRUGIRVVIVVMAOTOP LUG IRTP P XQRFRP SHMAQW

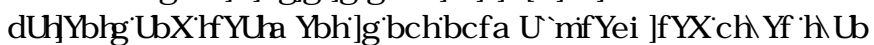

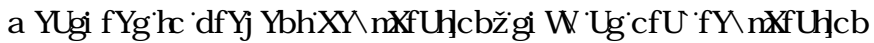

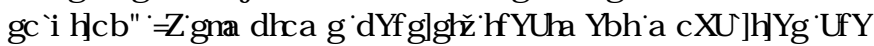

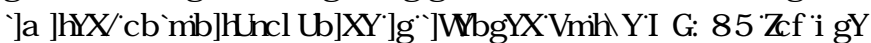

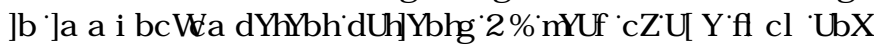

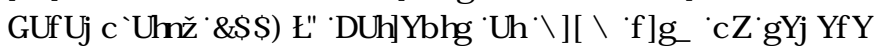

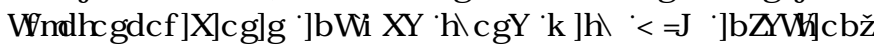
OXNHP LIDQGQQP SKRP DUSDUWFXOUQ FFKLGUHQUIRUYKRVH

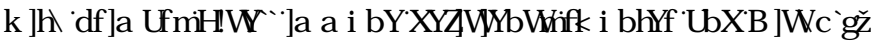

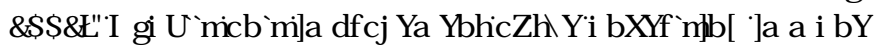
FRQGURQZHHXQVIRTMI QUIFDQMMP SLRYHP HQMGYKKH[SUP DU WHDVF HQMMRLIP P XQRVXSSUHMHG[SDWHOWLVUHGXFURQRU

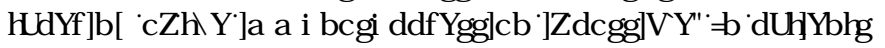

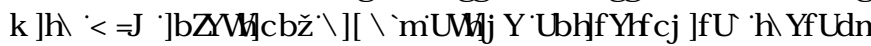

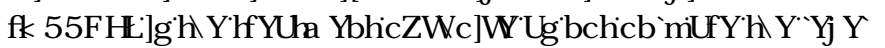

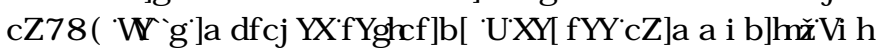

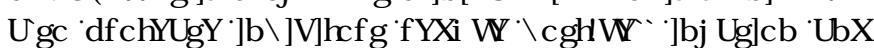

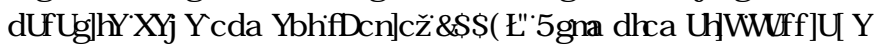
RFFXUMLQERUXIP P XQRFRP SHMAQVDQGIP P XQRFRP SLRP LMHG

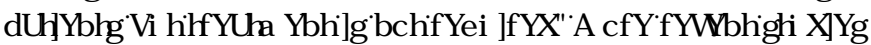

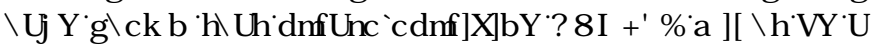
SLRP LVQ] DDOMIFU SURVSRUGDOGUX] [FDQGGDMAZLLKLDFUYLW D) DLQWVERVK $\square C$. parvum DQG $\square$ C. hominis $\square[8 \mathrm{QQNH}$

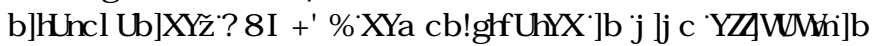

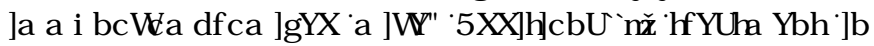

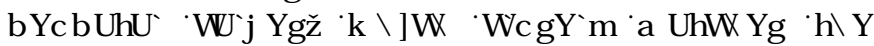
SDUKRSKI URQI IFDQDQGSKDUP DFRQI IFDQFKDCOI HMIDFHGLQ

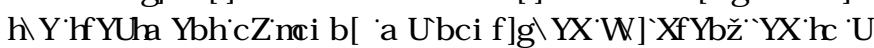

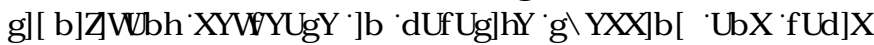

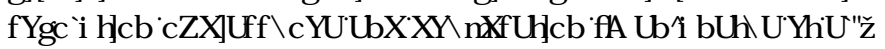
प्रापा

\subsection{Environmental Occurrence and Persistence}

7KHUHDDUH[QXP HURXVDSSURDFKHMDRUVIKH[GHMFFURQRRI Cryptosporidium $\amalg \& R P$ SDUVRQTRIUXGHMDDQGXQGHUWDGLQ

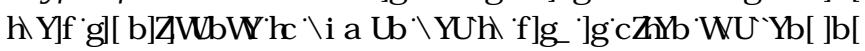

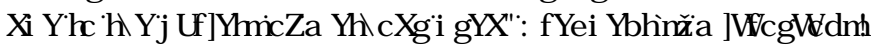

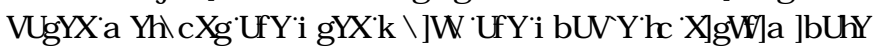


KXP DQSDMRRI HQFIURP [DQP DQDWRFLMAGCryptosporidium

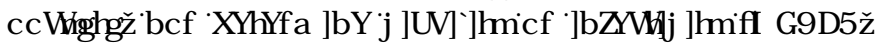

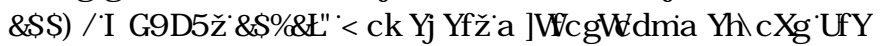

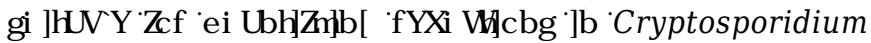
FRQFHQWDURQVD] LQDQQ DUHFHQVQ [GHYHORSHG IP HMRGV

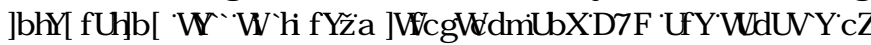

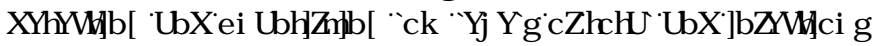
KXP DQSDMKRJ HQF[Cryptosporidium RRF WMII DODFHMALM DQm

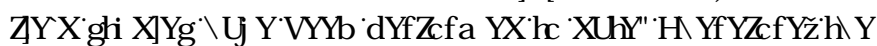

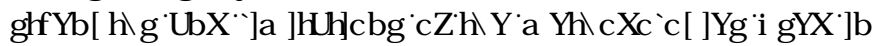

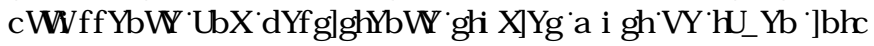
FRQMGHDMRQT

\subsection{Detection Methods}

6VDQGDCP HMRGYIRUIMYHGMMFURQDQGPQXP HDMRQR Cryptosporidium RRFI WWLZZDMUDQG HQYLRQP HQVDO

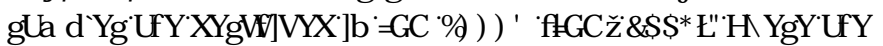

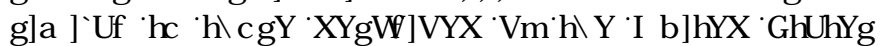

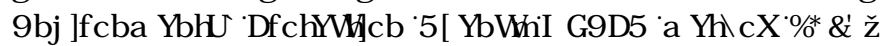

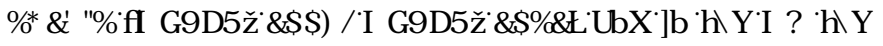

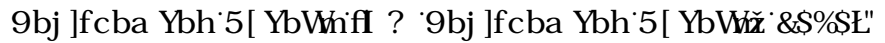

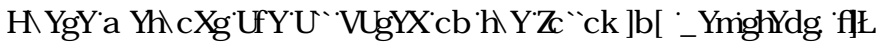

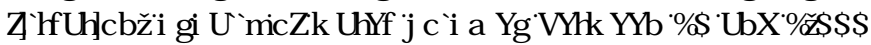

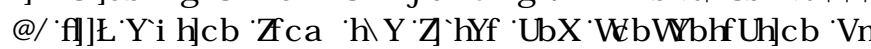

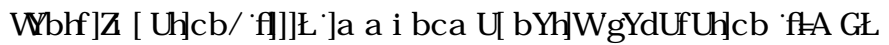
XVG [SDUDP D QHMFEHDGMFRDMAGLLKLUSHFLIFLOMERGH

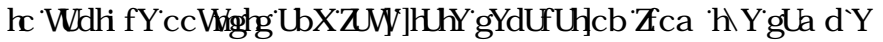

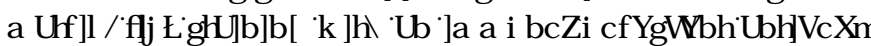

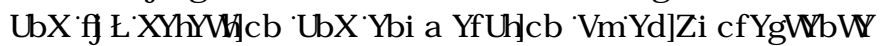

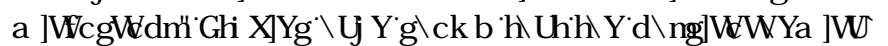

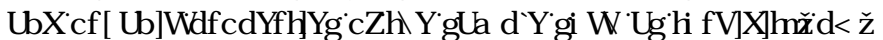
VKH [SUHMHQFHRRI LQVAU HUQ] [SDUMFONDDQGLQRY DQF

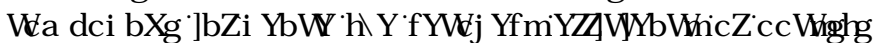

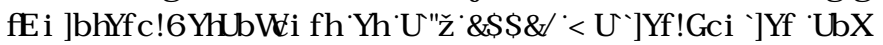

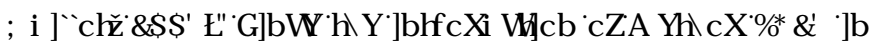

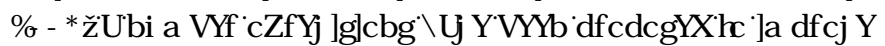

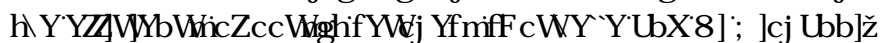

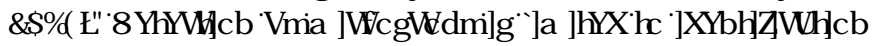

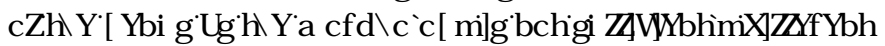

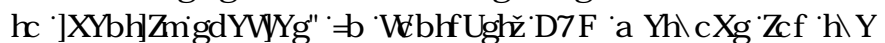
GHVHFVRQ RI [NRVDODDQG [YLEOH [KXP DQ[SDVKRJ HQLF Cryptosporidium VXFK DVVCryptosporidium [SURSLGIXP

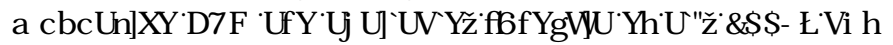

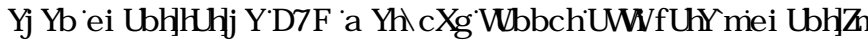

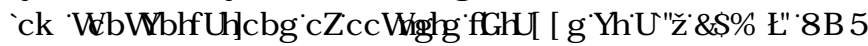

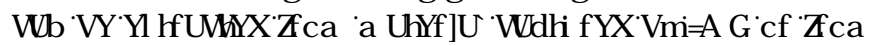

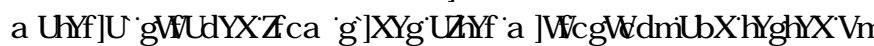
P ROFXOUP HMRGVI/ HEKHYDCHUHDQ

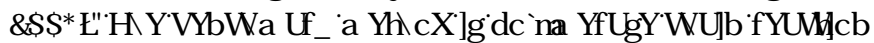

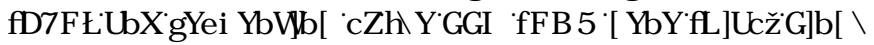

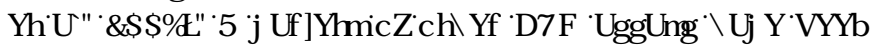

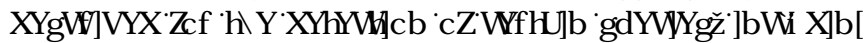

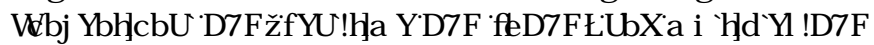

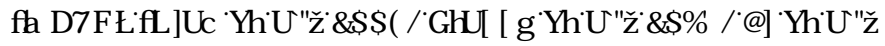

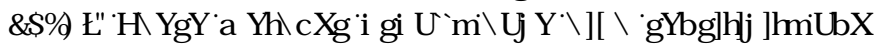
VSHFU LIWDEXXVYKHLUSHU RUP DQFH RQ HQYLRQP HQVDO

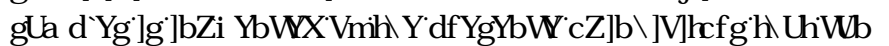

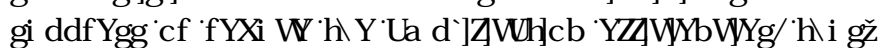

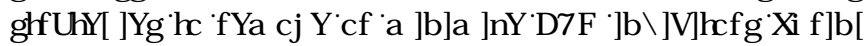

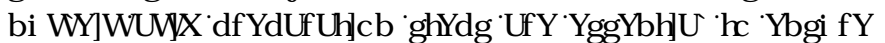

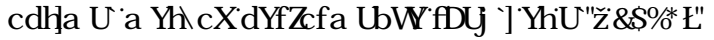

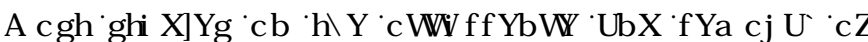
Cryptosporidium KDYHXXD] HGP IFURVFRS [DQGB\&5[DWA VD Z KLFK [GR [QRW/GHMHUP LQH [YLELOW RU RQI HFWYLW

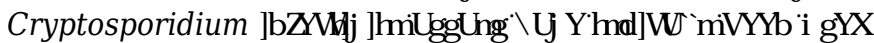

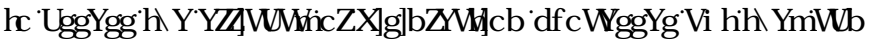

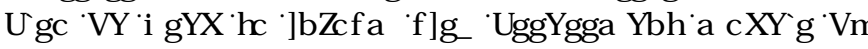
GHMALP LQL IYKHLQ HFUYLW RI RRFI WLQLHQYLRQP HQVDO

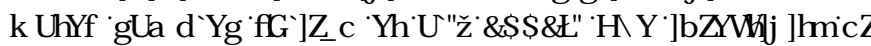

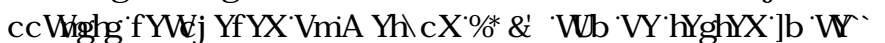
FXOXUHDWD VEI IP RGIIFDMRQRILMHRRFI WPP D QHMFEHDG

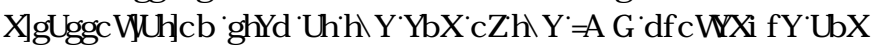
RP LWQ IP IFLRVFRSIFIH DP LQDURQRIRRF WWII/ H\&KHYDOH

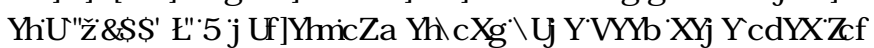
GHAFWQ IQ HFWRQLQFHQF XOXUHDDQGDWD VRDYHEH

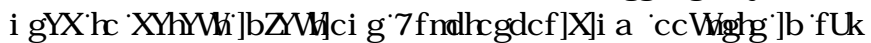

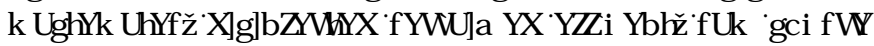

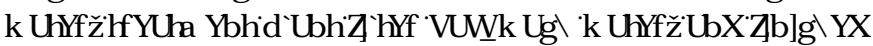

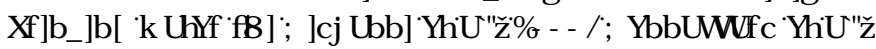

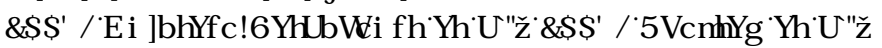

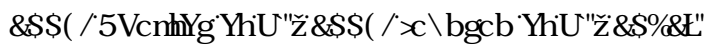

\subsection{Data on Occurrence}

7KLVMYFWRQEUH Q[VXP P DU]HMLMH] REDQRFFXUHQFH R[Cryptosporidium RRF WMLQXLEDQYZD HWXUDFHZDMA]

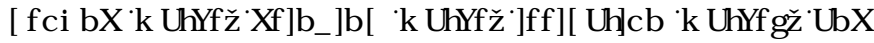

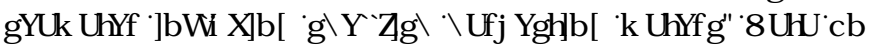
HQYLRQP HQVDOSHUMWAQFHRI [Cryptosporidium LVDQR

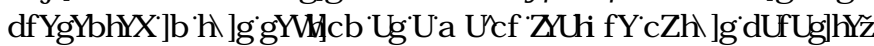

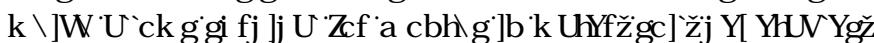

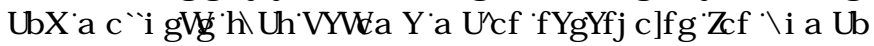
LIHWRQ]

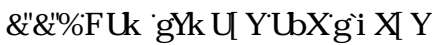

( QGP IF[OMHQYRIFU SURVSRUGIRVMLQP RWLSRSXOMRQV OHDG IVR IVKH I UHTXHQWZ RUGZ LGH IRFFXUHQFH LRI Cryptosporidium RRF WLLUDZ [M-ZD HШDDEOM [SURYLGN WHP RWFXUHQMFRP SUKHQVYHGDMDIRU] REDQRFFXUHQFH R[Cryptosporidium RRF WLLUDZ[M-ZD H四] HQHDQ]MKH RFFXUHQFHRI [Cryptosporidium RRF WWRQXUEDQRM-DD H

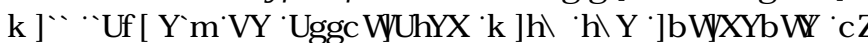

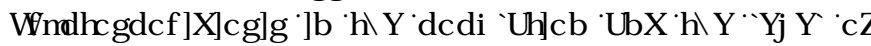
VDQVDURQLYHUYFHNDYDLCEOHBUHYDOQFHUDMALEDMGRQ

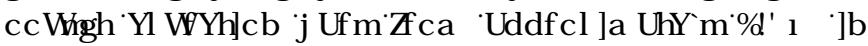
LQGXWDDD] HGFRXQWHM

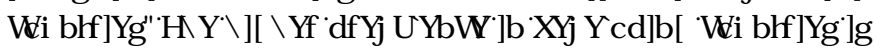

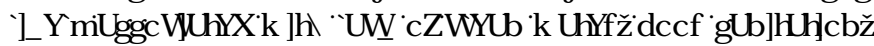
FURZ GHG TKRXVQ] FFQGLRQVIDQGF RVHUTFRQVFWVZ LVK

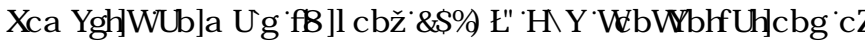
Cryptosporidium LQYHZD HDQGFRUHSRQGQ IUP RYDQE।

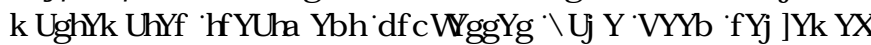

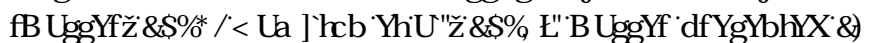

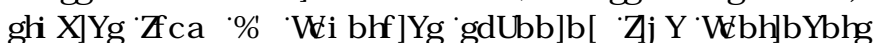

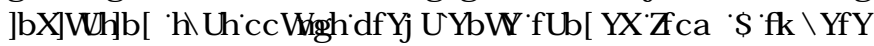
VDP SOMFRQWDQHGRRFI WMEHORZ [MKHGGMAFWRQ [OYHQTIR

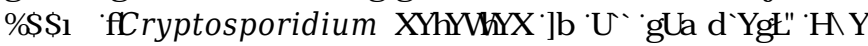
DYHUD H[SUHDDOQFHRR [Cryptosporidium RRFI W'QQ]DZ

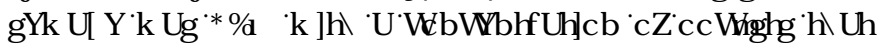

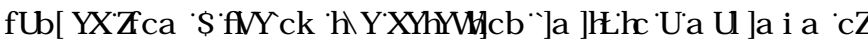




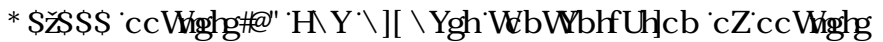

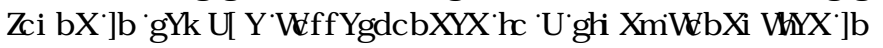

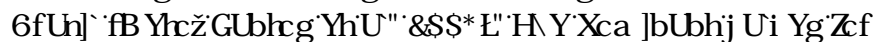
UKHFRQFHQWDMRQRI [Cryptosporidium LQRDZ [M-ZD] H

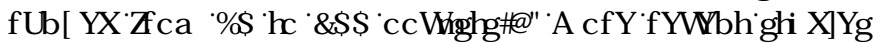

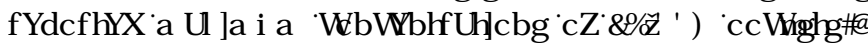

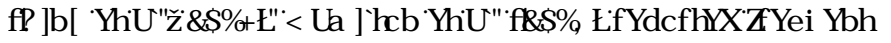

GHMAFURQRI [\&U SVRVSRUGLXP TQRM-ZD HIDQG[WHDMAG

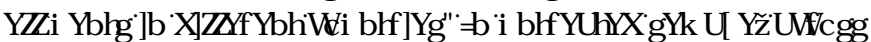

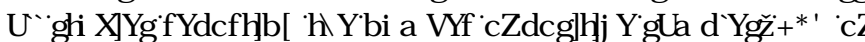

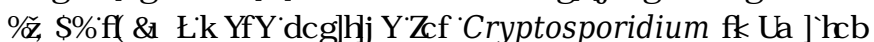
HMDQ

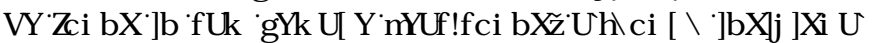
WXGHMKDYHUHSRLAGGGIIHHOWUY-DVRQDQSHDNRFFXUHDFHC

Table 2. Frequency of detection and concentration of Cryptosporidium in untreated sewage

\begin{tabular}{|c|c|c|c|c|}
\hline Country & Sample Type & Percent Positive Samples & $\begin{array}{c}\text { Concentration } \\
\text { Average (Range) Oocysts/L }\end{array}$ & Reference \\
\hline$\$ X W 1 D O D$ & 6HZD H & 15 & 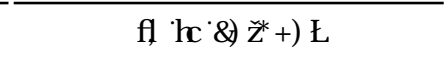 & 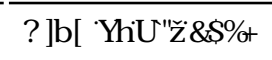 \\
\hline 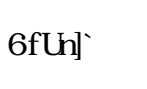 & $5 D Z[Y-Z D \mathrm{H}$ & प्राप्रा & 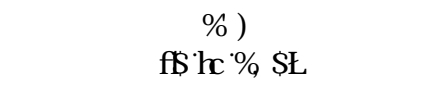 & 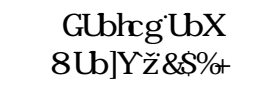 \\
\hline 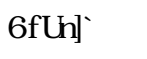 & $5 D Z[Y-Z D \mathrm{H}$ & पाण्रा & 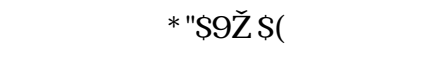 & 1 HMLMDQR \\
\hline$\% Х Ф D U D$ & 6HZD H & 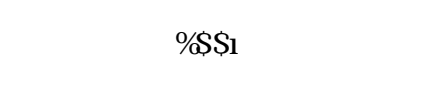 & 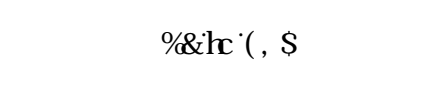 & 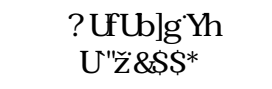 \\
\hline \&KLQD & 6HZD H & प्राप्रा & 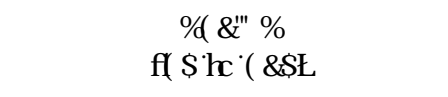 & ; LRTHDDQ \\
\hline * HP DQ & 6HZD H & प्याप्रा & 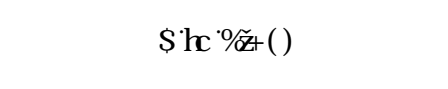 & 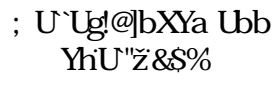 \\
\hline +XQ DU & 6HZD H & 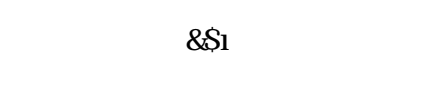 & (1) & 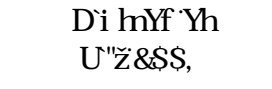 \\
\hline,$\quad 100$ & 6HZD H & 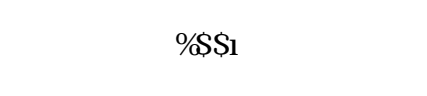 & 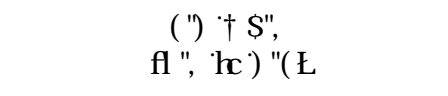 & 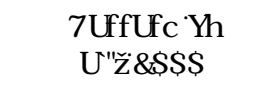 \\
\hline $1 \mathrm{RUZD}$ & 6HZD H & प्राप्र & 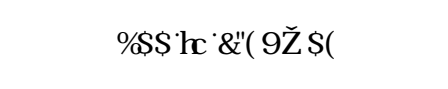 & $\begin{array}{l}\text { 5REHUMRQHM } \\
\text { DQm }\end{array}$ \\
\hline $\begin{array}{l}\text { 6RXVK } \\
\text { \$IUFD }\end{array}$ & 6HZD H & 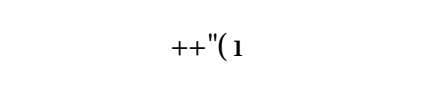 & 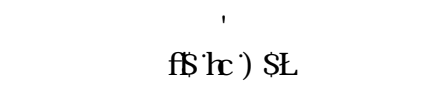 & 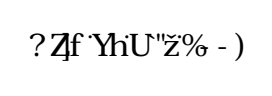 \\
\hline 6SDQ & 6HZD H & प्राप्रा & 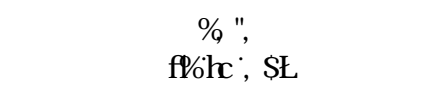 & 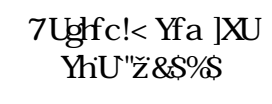 \\
\hline 6SDQ & 6HZD HRQQHQW & प्राप्रा & 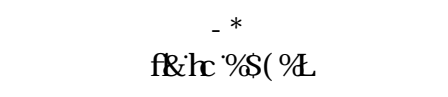 & 5DP R[MDDQ \\
\hline $\begin{array}{l}\text { 8QUAG } \\
. \text { LQ GRP }\end{array}$ & 6HZD H & 15 & 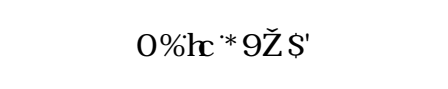 & $\begin{array}{l}\text { 5REHURQH } \\
\text { DQm }\end{array}$ \\
\hline $\begin{array}{l}8 \text { QUAG } \\
\text { 6VWAN }\end{array}$ & 6HZD H & 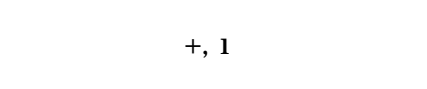 & 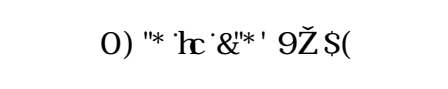 & $\begin{array}{c}\text { * HQQDFFDURTHW } \\
\text { DQdm }\end{array}$ \\
\hline
\end{tabular}

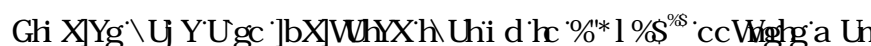

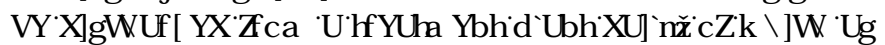

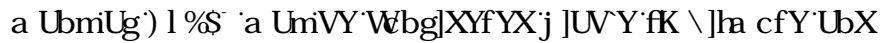

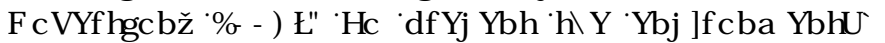
WDQNP LMRQR [Cryptosporidium IURP [M-ZD HTWHDVP HQW

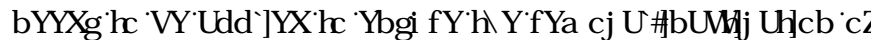

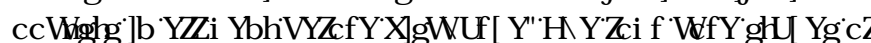
WHDVP HQWIQFOKHTLSUHQP LQDU TWHDVP HQVE LSUP DU

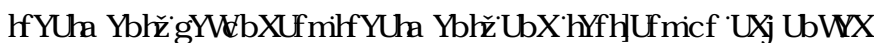
WHDV HQMVT KHTP DQRENAFUYHRIFRQYHQMRQDQZ DMAZ DUA

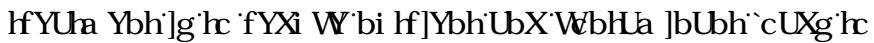

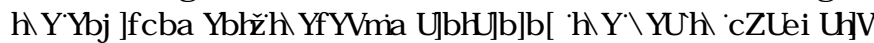

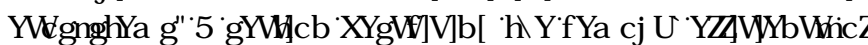

Cryptosporidium RRFIWW [E\ RDWHZ DVAU [WHDVP HQW

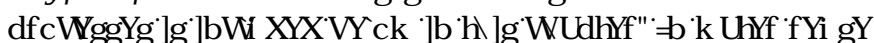
DSSOFDURQVIFRQYHQMRQDQNM-DVA HQMFDQEHIVXSSOP HQMAG ZLLK DDGLURQDQSURFHMHVIVR DFKLHYHDDTXDOW DMKDVIV

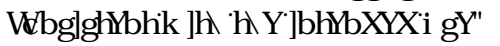

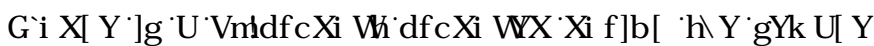

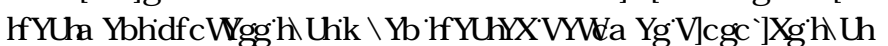

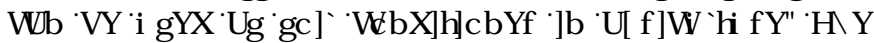
RFFXUHQFH[RI RRFI WV[KDVEHHQ[WXGHGLQ[VOXG H]URP

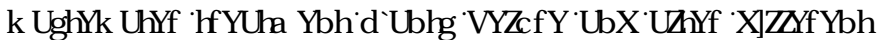

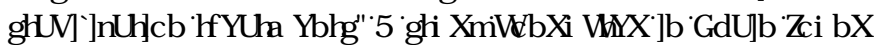

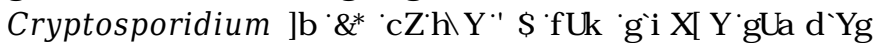

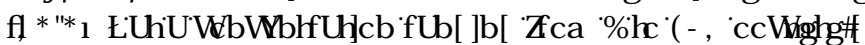


[\$P RUIVHMDQIس]

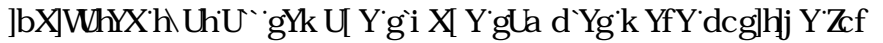
C. parvum [DQGC. hominis $\mathbb{Z}$ LMXP DI IP XP FRQFHQMDURQNR पIRRFI WW] [QR[SUP DU [VOG] H[QGLFDM] [MKH[QHHG]RU

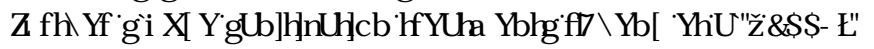

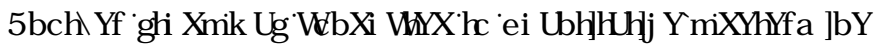
DQGFRP SDUHYKHFFRQFHQWDURQVRIYLEOCC. parvum [DQGC. hominis [RRFI WWLQ[M-ZD] H[VOXG] H[GXUQ] [MKH[DFUYYDMRQ

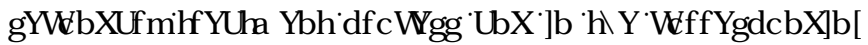

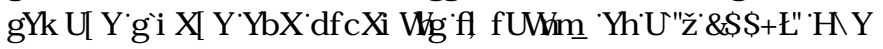
KL KHWVFRQFHQWDWRQ RI CCryptosporidium ZDV

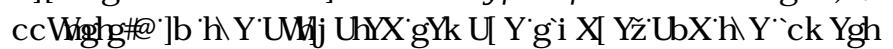

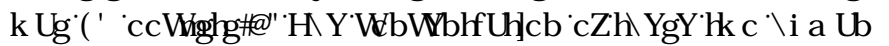
SDMKR] HQMLQGGZDUAHGDQG[EIRQIJ IFDQ [MDELD] HGMUZD H

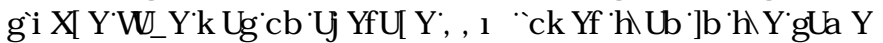

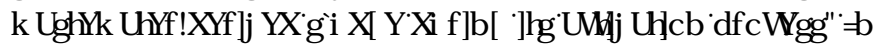

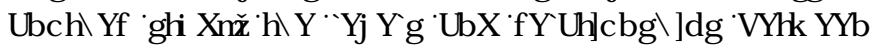

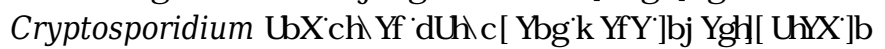
ELRVRQGV TURP RLQH [Z DWHZ DVHU LWHDVP HQWSOQW WXLRXJ KRXVYMKH[8 QLAGG GVDMAMTCryptosporidium RRFI WW

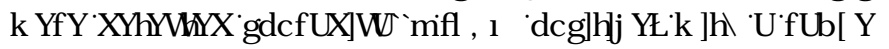

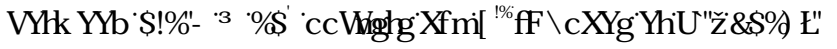

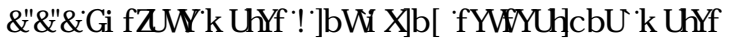

$7 \mathrm{KH}$ 口HWUP DVHG Q REDOP HDQ [SUHYDOHQFH QRU

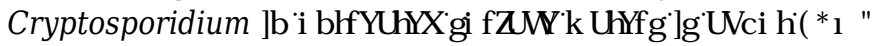

7KHFRQFHQWDURQRI Cryptosporidium LQJXUDFHZZDUAMTMD

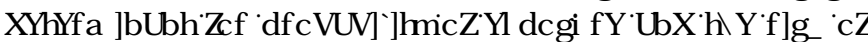

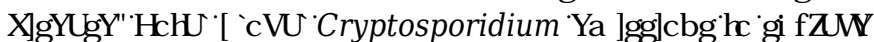

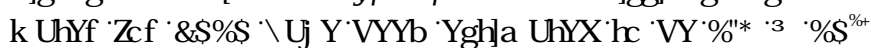

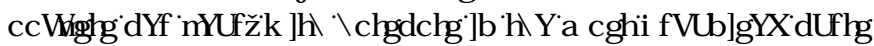

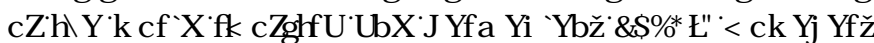

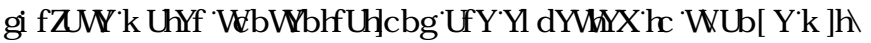

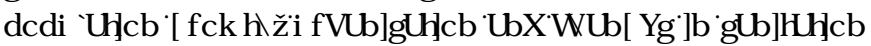

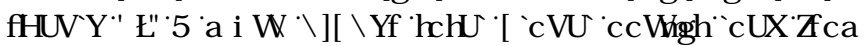
DQP DQP DQXUHRI

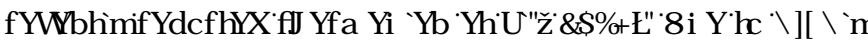

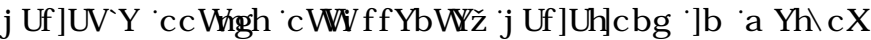
UFFYHUHMDDQGWKH[HIIFWRI GGUIHUHQWR DUAUP DMFHMRQ

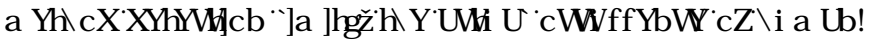
SDUKR] HQLF[Cryptosporidium RRF। WLRQLRXLFH[Z DUAUTV

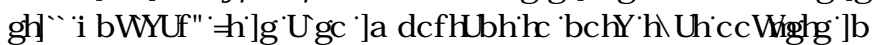
VRXUFH[Z DMALVIP D [EH[GHUYHG]URP P XOUSOILMRXUFHM LQFOGQ IKXP DQVWOYHMRFNDDQGZ LEOHWD DRRUIRXUFHMIRU SURVR] RDQ[FRQVDPP LQDMRQRI [VXU DFH[ZDMAUTOF $X G H[M K H$

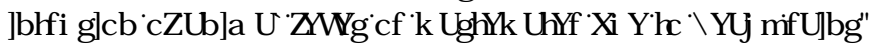

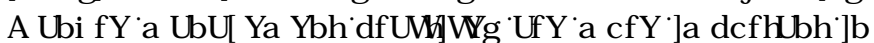

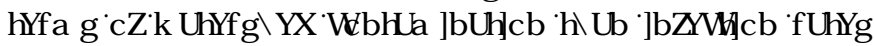

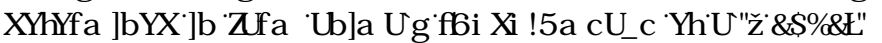
6LOFHPP DQ [RI [MKH[Cryptosporidium VSHFLHMDQG] HQRWSHN DWRFLDUAGZ LUK[DQIP DOMDUH[QRVV RRQRWF TKXP DQRKHDOK

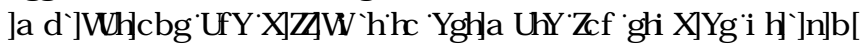
,) \$DRUYKH GGMAFURQRICryptosporidium RRF WMLQJYXUDFH Z DUAMID RUHRYHUT) \$ [GRHMLRVWSLRYLGHRQI RUP DMRQRQ RRF WOYLELOWD

Table 3. Occurrence of Cryptosporidium spp. in surface water and recreational waters

\begin{tabular}{|c|c|c|c|c|}
\hline Country & Sample Type & $\begin{array}{c}\text { Percent Positive } \\
\text { Samples }\end{array}$ & $\begin{array}{c}\text { Concentration } \\
\text { Average (Range) Oocyst/L } \\
\end{array}$ & Reference \\
\hline$\$ X W D O D$ & 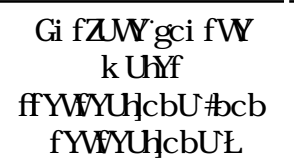 & 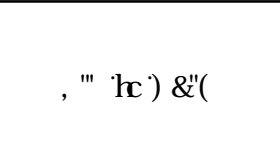 & 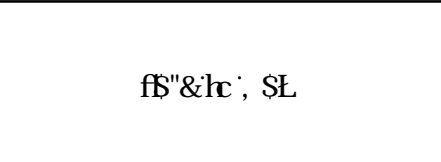 & 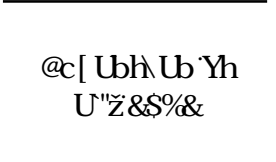 \\
\hline \%Ф ХXP & 6XE[FDUFKP HQW & 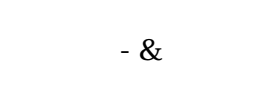 & 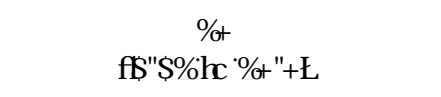 & 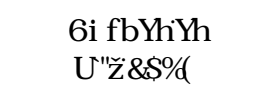 \\
\hline \%Ф ХХР & 5LHU & प्र & 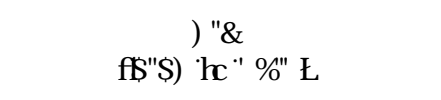 & 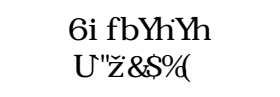 \\
\hline \%Ф ІХР & 5HMHYRU & प्र & 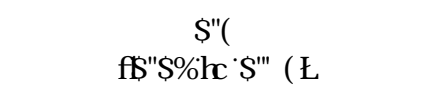 & 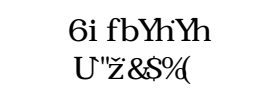 \\
\hline \%Ф ХХP & ' : 73ДQ9нм & प्र & 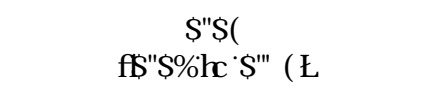 & 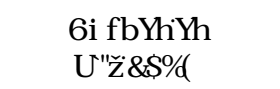 \\
\hline 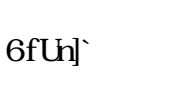 & $\begin{array}{l}\text { 6XUDFHURXUFH } \\
\text { ZDUAU }\end{array}$ & प्णा & 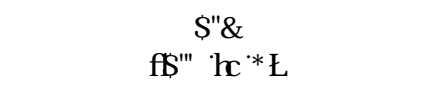 & 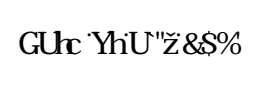 \\
\hline \%ХФDUD & 5LHYZDMA & प्र & एवसR⿴囗十 & 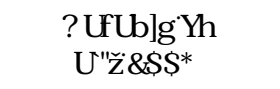 \\
\hline \&DQDGD & 5LHYZDMA & $\mathrm{am}$ & पाण्रा & 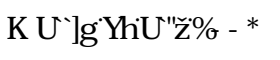 \\
\hline \&DQDGD & 5LHYZDMA & प्र & 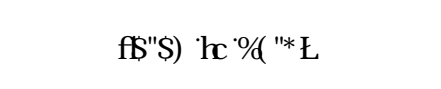 & 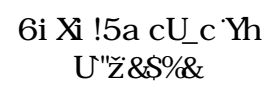 \\
\hline \&DQDGD & 7KUHZZDMAUKHGV & प्र & 15 & $\begin{array}{l}\text { 5XHFNHUHW } \\
\text { DQm }\end{array}$ \\
\hline \&KLQD & $\begin{array}{l}\text { 6XUDFHYRXUFH } \\
\text { ZDUAU }\end{array}$ & प्र & 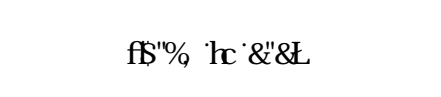 & 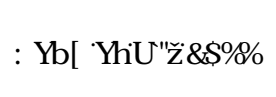 \\
\hline \&KLQD & 5HFUHDURQDQDDNHN & प्रा & 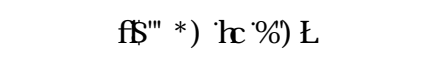 & 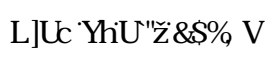 \\
\hline
\end{tabular}




\begin{tabular}{|c|c|c|c|c|}
\hline Country & Sample Type & $\begin{array}{c}\text { Percent Positive } \\
\text { Samples }\end{array}$ & $\begin{array}{c}\text { Concentration } \\
\text { Average (Range) Oocyst/L } \\
\end{array}$ & Reference \\
\hline ) LQDQG & 5LYHUDQGDNNA & 呬 & 15 & 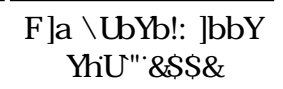 \\
\hline ) UDOFH & $\begin{array}{l}\text { 5HFUHDURQDO } \\
\text { VXUDFHZDUAV }\end{array}$ & प्र & 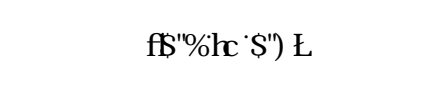 & 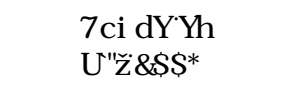 \\
\hline * HUP DQ & $\begin{array}{l}\text { 6XUDFHIRXUFH } \\
\text { ZDMU }\end{array}$ & 呬 & 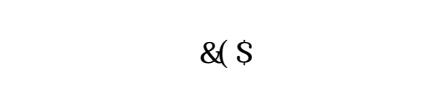 & 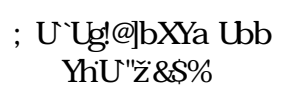 \\
\hline +Xg DU & $5 \mathrm{LYHV}$ & 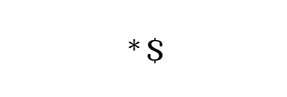 & 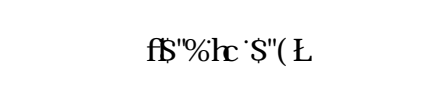 & 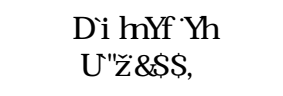 \\
\hline +xg Du & 5HMUYRLV & प्र & $\mathrm{am}$ & 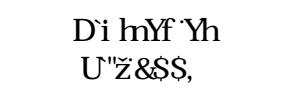 \\
\hline +Xg DU & \&DQDQ & 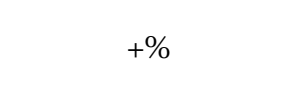 & 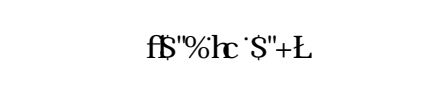 & 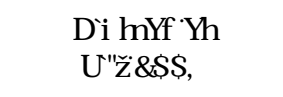 \\
\hline +xQ DU & 5HFULDMRQDQMUN & 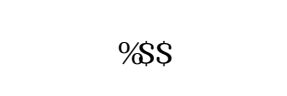 & 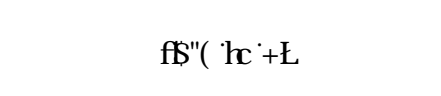 & 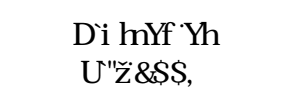 \\
\hline , UDQ & $\begin{array}{l}\text { 6XUDFHIVXXYFH } \\
\text { ZDMAU }\end{array}$ & $15^{\mathrm{D}}$ & 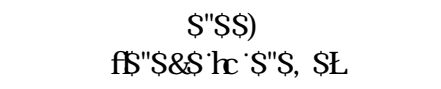 & +DGपMMQ1] \\
\hline, 100 & $\begin{array}{c}\text { / D RRQIRU } \\
\text { КHНOLKIDP LQ }\end{array}$ & 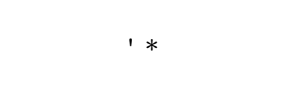 & 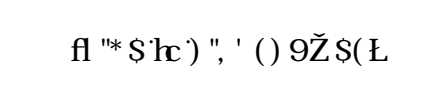 & 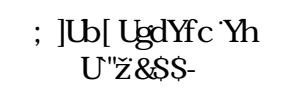 \\
\hline, 100 & $\begin{array}{c}\text { 6XUDFH } \\
\text { ZDMAUWYH } \\
\text { XSWH-DP }\end{array}$ & प्रा & 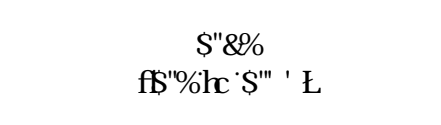 & $\begin{array}{l}\text { \&DUDRRHN } \\
\text { DQ }\end{array}$ \\
\hline, 100 & $\begin{array}{c}\text { 6XUDFH } \\
\text { ZDMUШGRZQMHDP } \\
\text { RILHIQHQW } \\
\text { GLFKDY H }\end{array}$ & 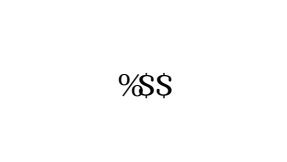 & 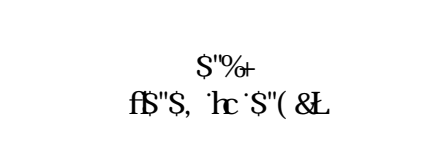 & $\begin{array}{l}\text { \&DUDRRHN } \\
\text { DQ }\end{array}$ \\
\hline 1 HSDO & 5 IYHZZDMAU & $1 \$^{\mathrm{E}}$ & 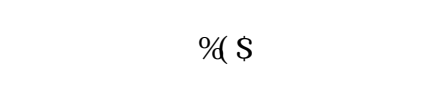 & $\begin{array}{l}\text { +DUP RVRTHW } \\
\text { DQm }\end{array}$ \\
\hline 1 HMHUDOQV & $\begin{array}{c}\text { \&DQDQDDQG } \\
\text { UFUHMRQDQQNNN } \\
\text { [HFUHDMRQDQ }\end{array}$ & प्र & 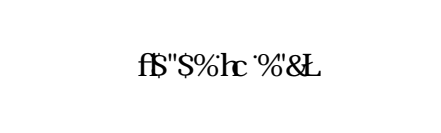 & 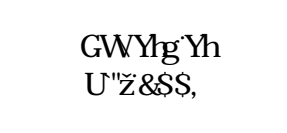 \\
\hline 1 HMHUDQGV & $\begin{array}{c}\text { \&DQDQDDQG } \\
\text { UFUHDMRQDQNHN } \\
\text { [QRQIJFUHDMRQDD }\end{array}$ & 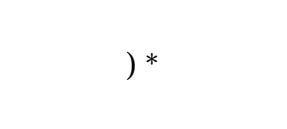 & 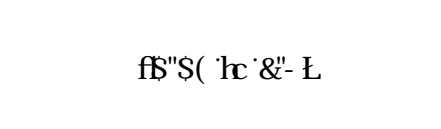 & 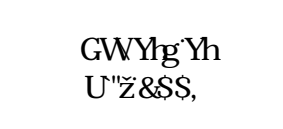 \\
\hline $3 \mathrm{HW}$ & $\begin{array}{l}\text { 5IYHUZDMAN } \\
\text { / RFDMRQW }\end{array}$ & 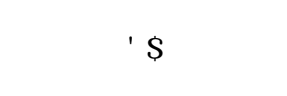 & 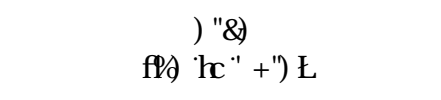 & 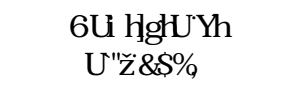 \\
\hline $3 \mathrm{HW}$ & $\begin{array}{l}\text { 5LYHUZDMAU } \\
\text { / RFDMRQW }\end{array}$ & प्र & 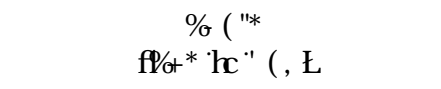 & 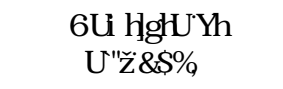 \\
\hline 3RUXJ DO & 5IYHUZDUAU & 品 & 15 & / RERमMDDQ \\
\hline 3RUXX DO & 5LYUZDMAU & प्र & 15 & 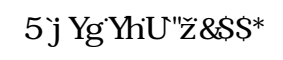 \\
\hline 3RUXJ DO & 5IYHZZDMAU & 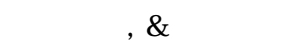 & 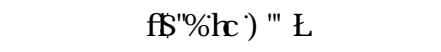 & 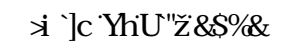 \\
\hline $5 \mathrm{RP}$ DQD & 5IYHUZDUA & $\mathrm{am}$ & 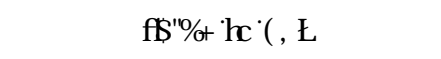 & 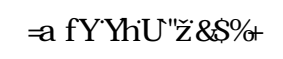 \\
\hline $5 \times M D$ & 5IYHZZDUAU & प्राप & 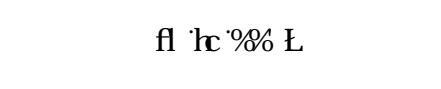 & 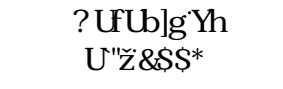 \\
\hline $\begin{array}{l}\text { 6RXVK } \\
\text { \$IUFD }\end{array}$ & $\begin{array}{c}\text { 6XUDFHIVXXFH } \\
\text { ZDUA }\end{array}$ & 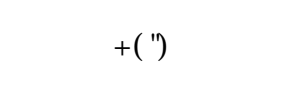 & 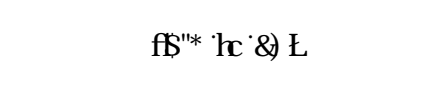 & 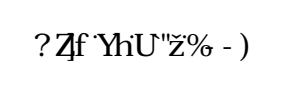 \\
\hline 6SDQ & $\begin{array}{c}\text { 6XUDFHURXUFH } \\
\text { ZDUMU }\end{array}$ & 밈 & 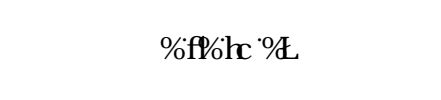 & 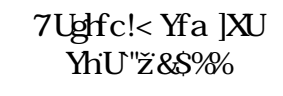 \\
\hline 6SDQ & $\begin{array}{c}\text { 6XUDFHZDUA } \\
\text { VRXUFHUFUHDMRQDO }\end{array}$ & प्रा & 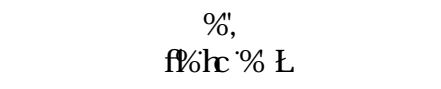 & 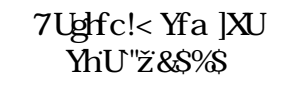 \\
\hline 6SDQ & $\begin{array}{c}\text { 6XUDFHIVXXFH } \\
\text { ZDUAU }\end{array}$ & प्र & 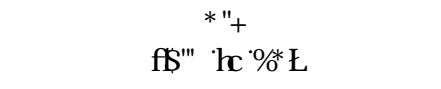 & 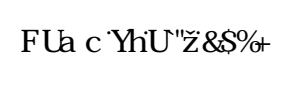 \\
\hline $\begin{array}{l}8 \text { QUAG } \\
\text { 6VWMN }\end{array}$ & ' : 73ДQ0ल & Q & प्राप्रा & 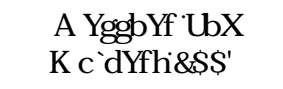 \\
\hline $\begin{array}{l}8 \text { QUAG } \\
\text { 6VUMN }\end{array}$ & $\begin{array}{l}\text { 5IYHUZDUAKHG } \\
\text { 5XUDDQGXLEDQ }\end{array}$ & 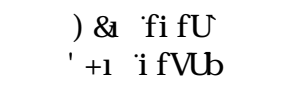 & 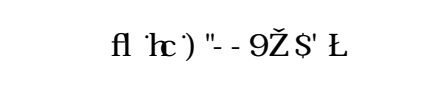 & 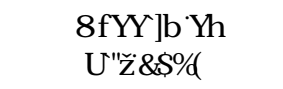 \\
\hline $\begin{array}{l}8 \text { QUAG } \\
\text { 6VDMAN }\end{array}$ & $\begin{array}{l}\text { 6XUDFHIRXUFH } \\
\text { ZDUA }\end{array}$ & प्र & 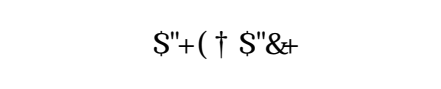 & 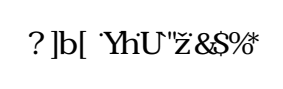 \\
\hline
\end{tabular}




\begin{tabular}{|c|c|c|c|c|}
\hline Country & Sample Type & $\begin{array}{c}\text { Percent Positive } \\
\text { Samples }\end{array}$ & $\begin{array}{c}\text { Concentration } \\
\text { Average (Range) Oocyst/L }\end{array}$ & Reference \\
\hline 9HQH XHD & ' : 73ДQOMN & प्र & 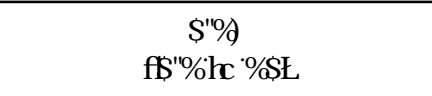 & $\begin{array}{l}\text { \%HDOFRXULDQG } \\
0 \text { HQD四D }\end{array}$ \\
\hline 9LHODP & 5LYH & पढ口 & 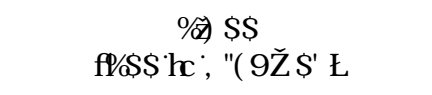 & 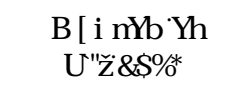 \\
\hline 9HМODP & : DMATFDQDQ & पढ口 & 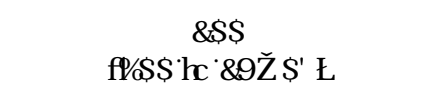 & 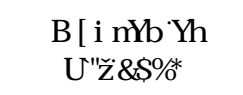 \\
\hline
\end{tabular}

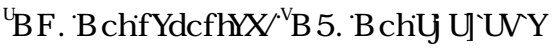

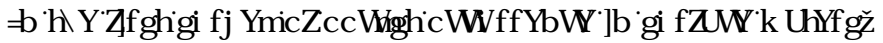

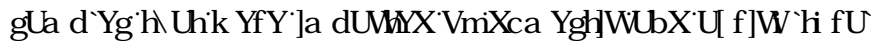
ZDWAIKDGCryptosporidium FRQFHQNDURQNDMKL KDM

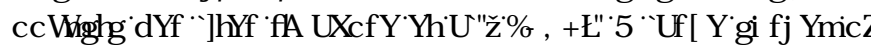

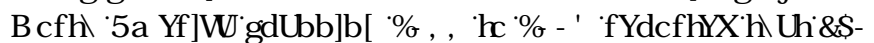

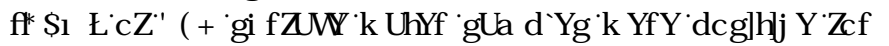
Cryptosporidium RRF WW

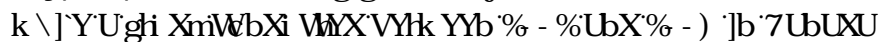

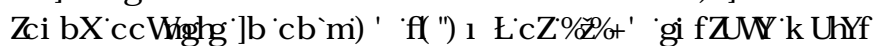

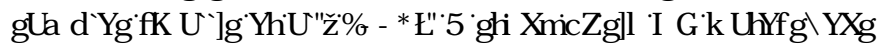

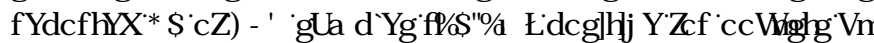
0 HKRG

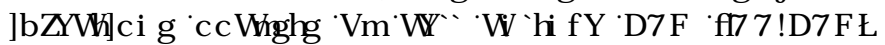

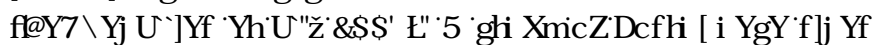

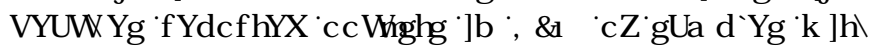

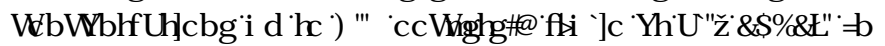

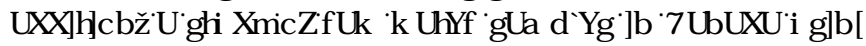

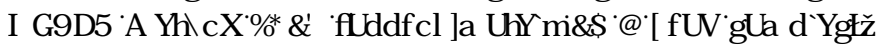

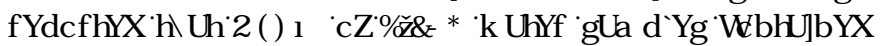

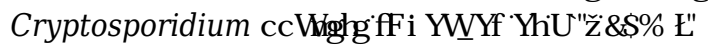

5H XOVRU [P RQLRUQ] [SUR] UDP VLWSLFDC [GHP RQWWDA

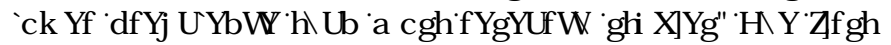
UH XOURU IP RQLRUQ [RICryptosporidium L88 6[ZDMAVZDV

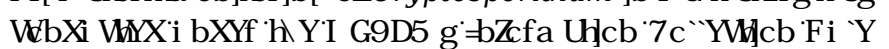

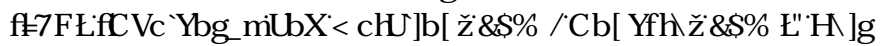

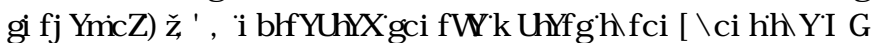

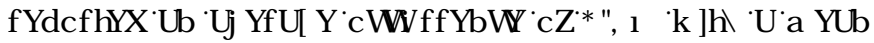

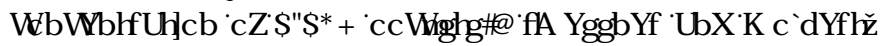

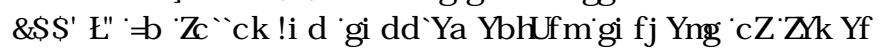

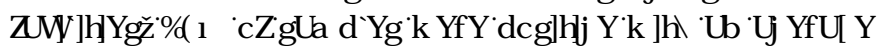

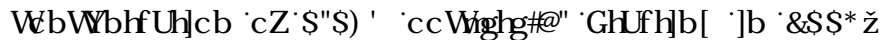

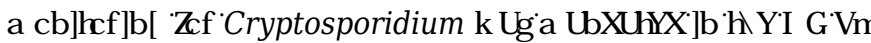

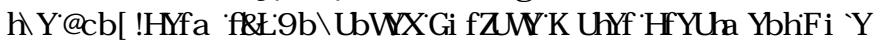

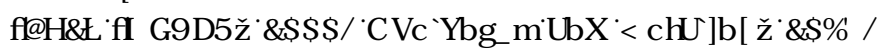

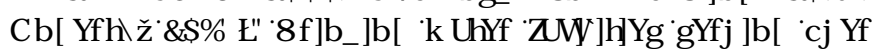

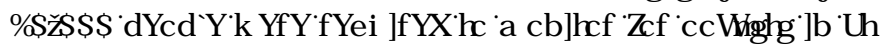
ODWMIIFRQMFXVYHP RQMKQLVP SOMRI IDZ ZZDMAXVG

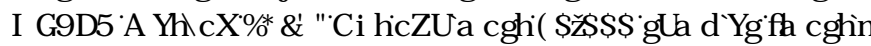

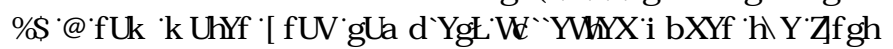

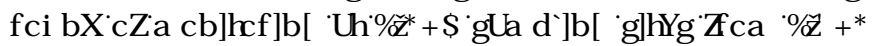
IDFLOMHMDFURWTMH 8 6ПMHYHQSHFHQMFRQNDQHGDMMODWW RQH[Cryptosporidium RRF WWR EROPMM DQG

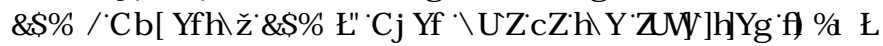
UHSRUAGGQR SRVWYHLMP SOMDDUDOUMLRXJ KRXVMKH[HQWLH

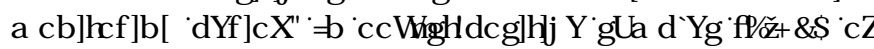

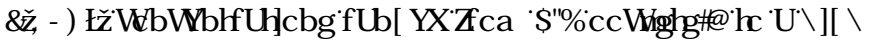

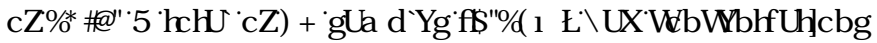

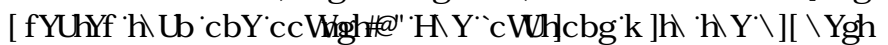

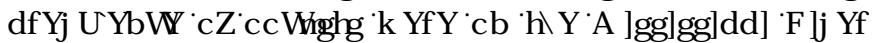

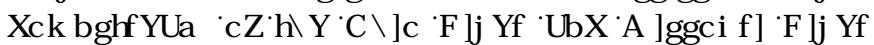

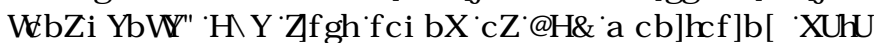

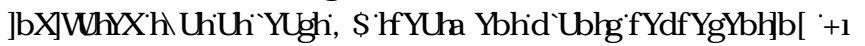

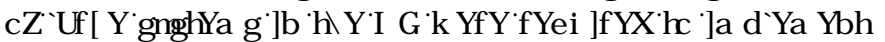
DGGLWRQDOMWHDVP HQWVR GQFUHDVHRRFI WUUHP RYDORU

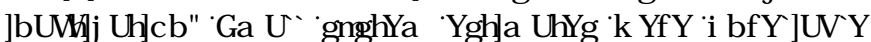

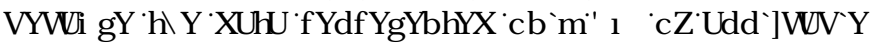

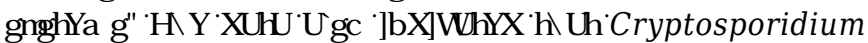
RFFXUHQFH[ZDV[KЦ KHUДQ ZDMALRXUFHMFRP SDUHGZZ LM UHMHURU[DNHWSHLXUDFH ZDMAU[MRXLFHN[RU] URXQGZ DMAU[XQGHU[GLHFWIQI ХHQFH

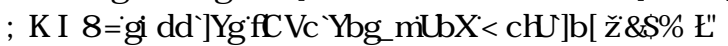

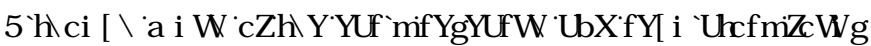
ZDVRQGUQNL] [ZDMAUCryptosporidium KDVEHFRP HपYKH

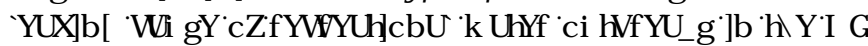
[+DVMLHDQ

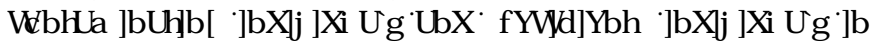
UFUHDMRQDQZDUAU TSDUFXOUQ IVZ IP P LQ [SRRQTP HDQV

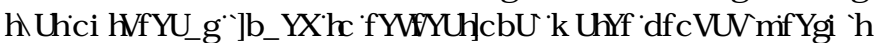

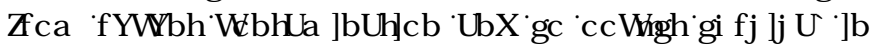

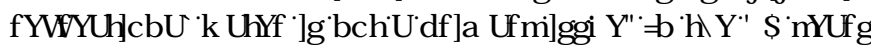
VSDQQL

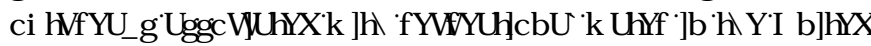

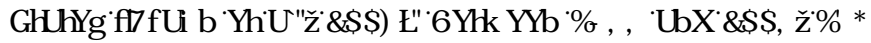
GRFXP HQVAG RXVEUHDNVLRI FU SVRVSRUGIRVV [OQNHG [VR UFUHDMRQDQXVHR ZZ DUALQRQQHFRXQMHMUFNHQHGDVMRVD $\mathrm{R}$ 四

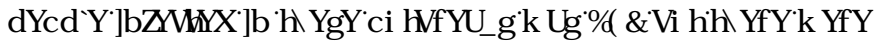

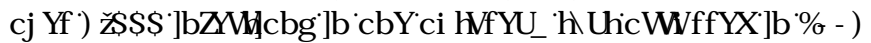

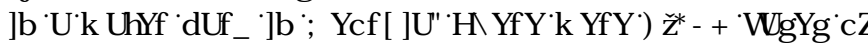

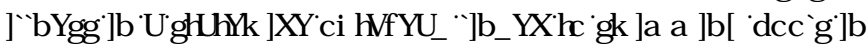

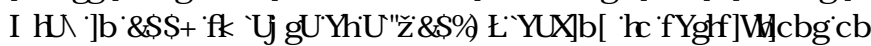

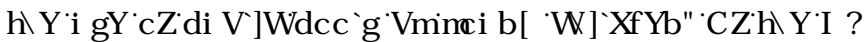

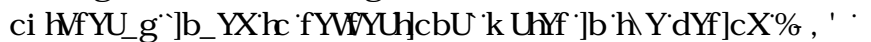

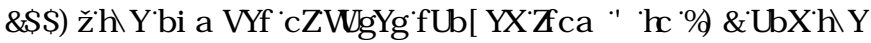

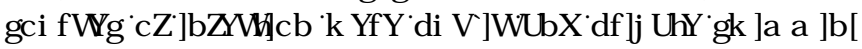

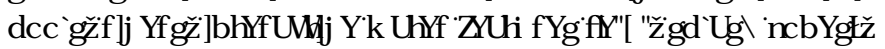

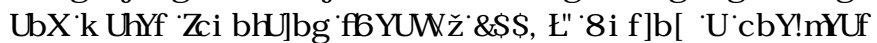

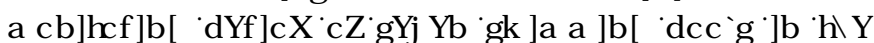

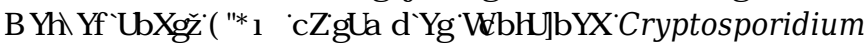
RRFI WVI\$GGLWRQDQLI RUP DMRQRI RXVZUHDWIDWRFIDUAG

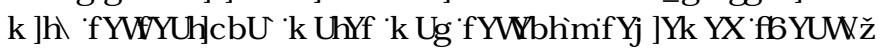




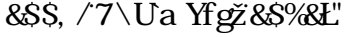

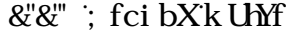

6XUYH VRI [RFFXUHQFHRI [Cryptosporidium LQQXP HRXV FRXQMHMLQGFDMALMKDMRRF WWRFFXURQTYRP H] URXQGZ DUA

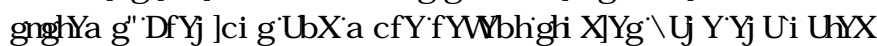
WKHSRMAQWDOIRUYMKHUDONSRUMRI C ryptosporidium WXLXJ K VRLOVIR [DQQG[GUDLQVIDQG] URXQGZ DVAULM WAP V[XVQ]

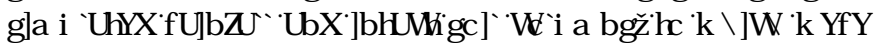

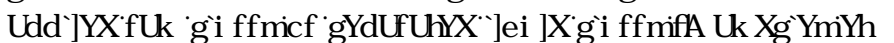

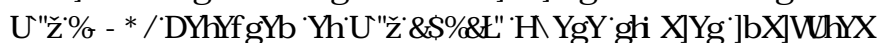
WKDMODFKLO] [RIRRFI WWGGZZ QJKKLRXJ K[YKYHLRLOSLRILOGGG

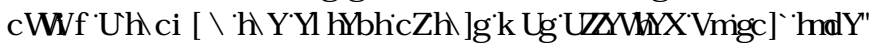
) RULOWDOFH IWDQVSRUWRI [C. parvum[RRFI WWMKLRX] K[URLO DQG]QMREOHDFKDMHZZ DV] UHDMHIQTDMON [RDP [DQGDFF(I)

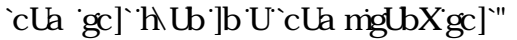

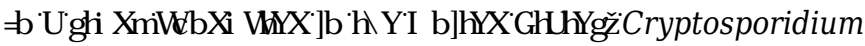

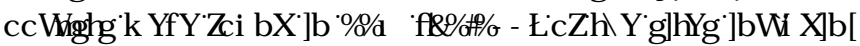

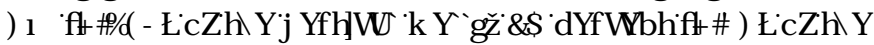

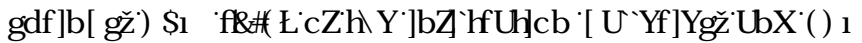

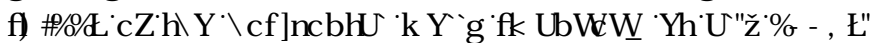

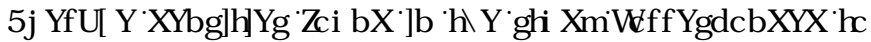

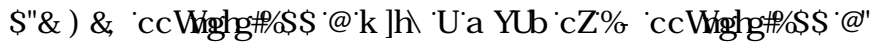

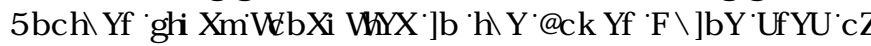
* HUP DQ IUHSRUAAG

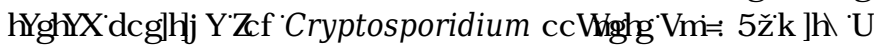

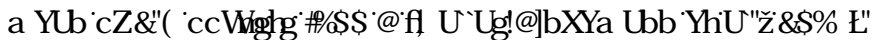
\$GGLURQDQWXGLHMRQLMKH[RFFXUHQFHRR [Cryptosporidium VSSПLQ] URXQGZ DMALM WAP VIURP [GLIHUHOMFRXQMHMDUH बMAGLOTDEOIII

Table 4. Occurrence of Cryptosporidium oocysts in groundwater systems

\begin{tabular}{|c|c|c|c|c|}
\hline Country & Sample Type & $\begin{array}{l}\text { Percent Positive } \\
\text { Samples }\end{array}$ & Concentration Average (Range) Oocysts/L & Reference \\
\hline \%ХDDD & $\begin{array}{c}\text { * URXQG DMAU } \\
\text { ZHФ }\end{array}$ & प्र & पालाप & 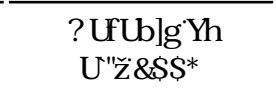 \\
\hline \&DQDGD & * URXQGZDMA & प्र & 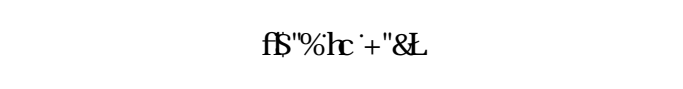 & 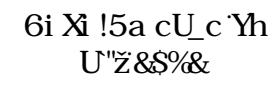 \\
\hline * HP DQ & $\begin{array}{l}\text { 5DGDQDQG } \\
\text { YHUFDQZ HCO }\end{array}$ & पाली & 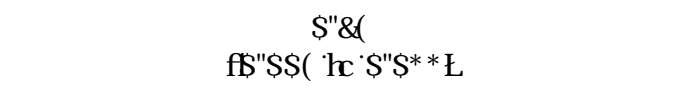 & 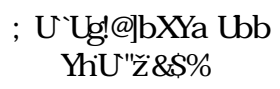 \\
\hline , QGD & ' HSZZHOV & $\mathrm{QP}$ & 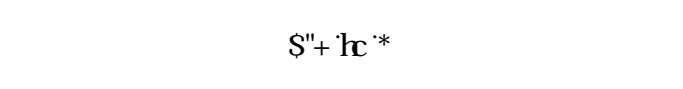 & $\begin{array}{l}\text { ' DQHOLHW } \\
\text { DQm }\end{array}$ \\
\hline , QGD & 6KDCRZZZHCN & 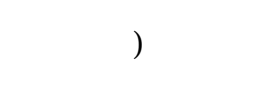 & प्राश्य & 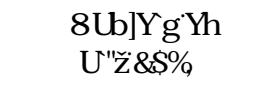 \\
\hline $1 \mathrm{HSDO}$ & $\begin{array}{c}\text { * IRXQGZ DMAU } \\
\text { Z HOV }\end{array}$ & $\mathrm{Cl}$ & 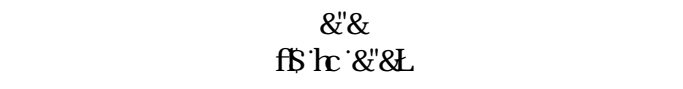 & $\begin{array}{l}\text { +DUPP RVRLHW } \\
\text { DQm }\end{array}$ \\
\hline $1 \mathrm{RLZD}$ & $\begin{array}{c}\text { * IRXQG DMAN } \\
\text { ZH@O }\end{array}$ & 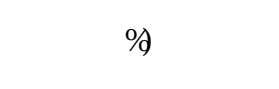 & aा & 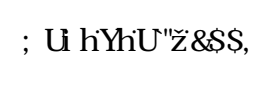 \\
\hline 3RUXX] DO & $\begin{array}{c}\text { 7UHDMAG } \\
\text { J URXQGZ DMA } \\
\text { IRU } \\
\text { FRQNXP SWRQ }\end{array}$ & प्र & 15 & 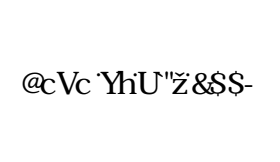 \\
\hline $\begin{array}{l}8 \text { QUAG } \\
\text { 6VIVAN }\end{array}$ & 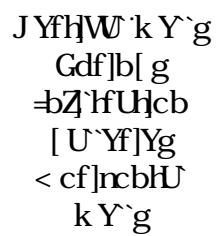 & 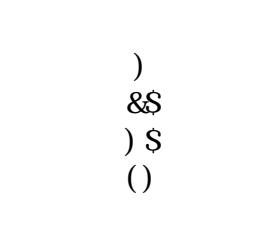 & 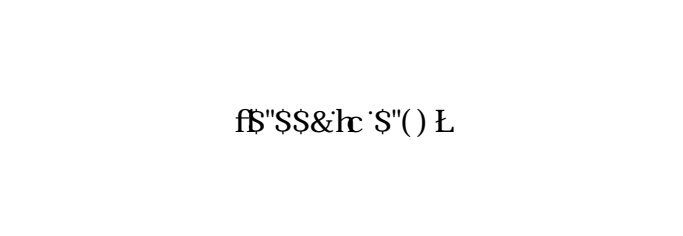 & $\begin{array}{l}\text { +DQFRFNHHW } \\
\text { DQ }\end{array}$ \\
\hline $\begin{array}{l}8 \text { QUAGG } \\
\text { 6VMAN }\end{array}$ & 6KDCRZZZHCN & प्र & 15 & 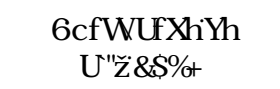 \\
\hline
\end{tabular}

15 [1 RWD HSRUAG

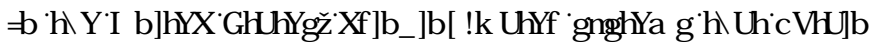

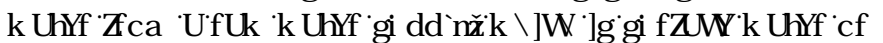

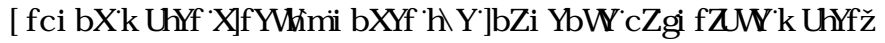

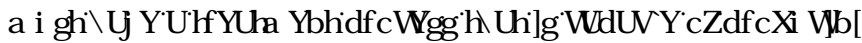
Z DUARR HTXDQRUEHMAUTXDOWLMKDQDTRP ELQDWRQRIZ HO
RSHUDAG]FKHP IFDQ TDMUAG LQWDURQDDG GLMQ HFWRQ SLRFHMHVZZ RXG[SLRYLGHTKLVLWHDVP HQVVSLRFHWL XWV DFKLHYHDQRYHDOSHURUP DQFHUMDLGLRYLGMDUDP LQP XP

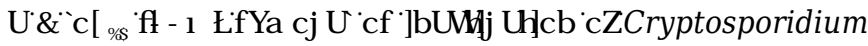
RRF WM 


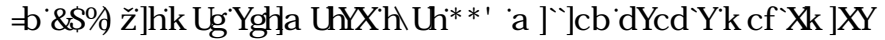
WUXVH[XQP SLRYHG[GUQNL] [ZDUAUIRXLFHMDQFOGL]

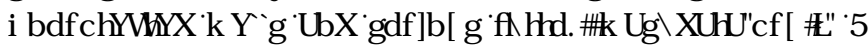

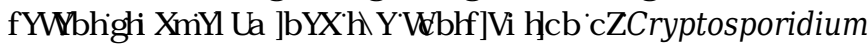

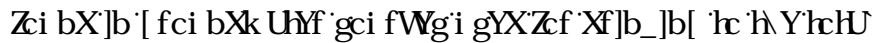

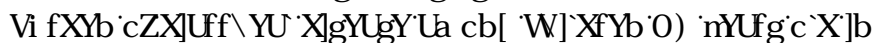

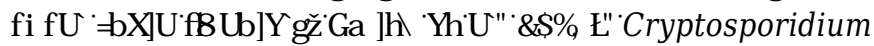
RRFI WWZHUHGHAFWGLQT]

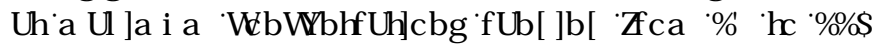

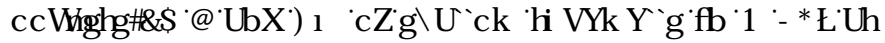

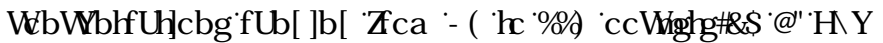

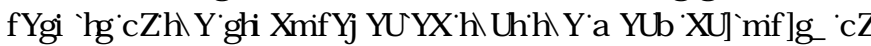
Cryptosporidium RQ H FURQ

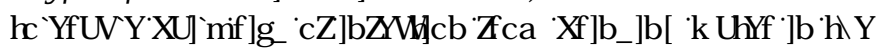

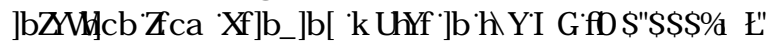

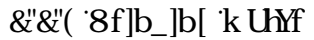

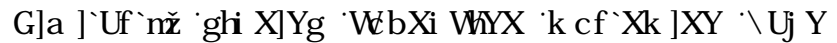

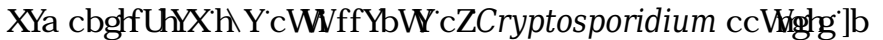

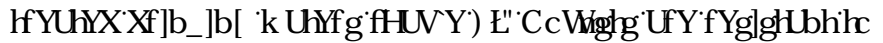
FKQRUQHGMQ IFURQDDUMKHFRQFHQNDURQVWSLFDQ DSSOHG

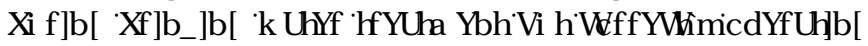

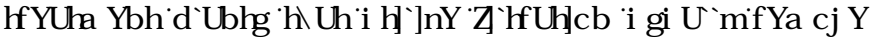
RRFI WW]URP [MRXUFH[ZDMAUI5 RFKHCHIDQG] L* IRYDQQL]

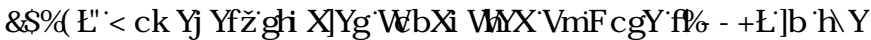

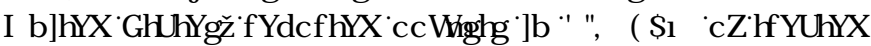

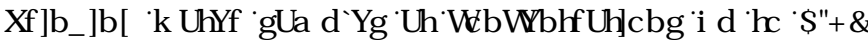

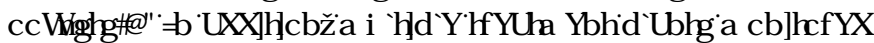
LQ] LVFRQMQ[GHMFUAGRRFI WVIQWس

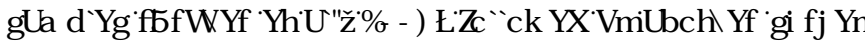

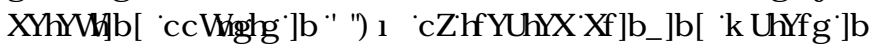

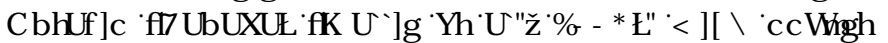

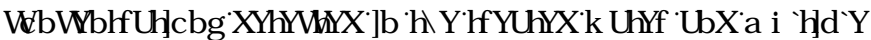

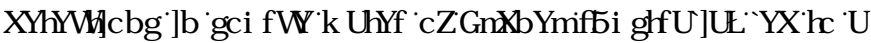
SHURGRIRQID DQQIRIID DQRERLQZDMAIDGLVRUHMRYHUYZR

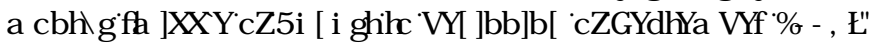
7KH [QFLGHQWIKDG [RQ] [DDO] LO] [SRQWFDQ[MHFKQLFDQ RSHUDMRQDQIDQGIP DQD HUDOFRQMTTXHQFHVRQ \$XVWDOD

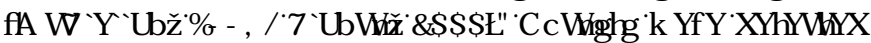

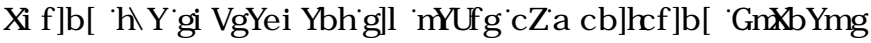

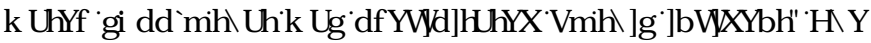

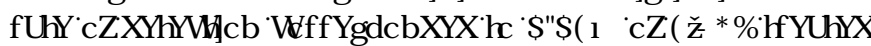

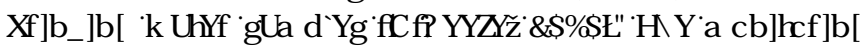
SHURGZZDFFKDDFWAU HGEI DOPH WAP HP XQWZ HDUGRXJ KW

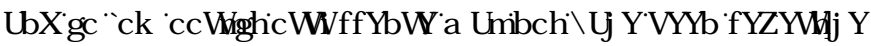
RIIQRLP DQIIFRQGMRQV]

Table 5. Occurrence of Cryptosporidium oocysts in treated drinking waters

\begin{tabular}{|c|c|c|c|c|}
\hline Country & Sample Type & $\begin{array}{c}\text { Percent Positive } \\
\text { Samples }\end{array}$ & Concentration Average (Range) Oocysts/L & Reference \\
\hline 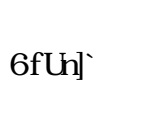 & $\begin{array}{l}\text { 7UHDMAG } \\
\text { GUQNG } \\
\text { ZDMA }\end{array}$ & प्रा & 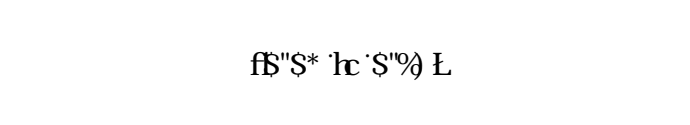 & 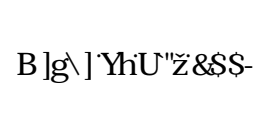 \\
\hline ما & $\begin{array}{l}\text { 7UHDUAG } \\
\text { GUQNG } \\
\text { ZDMAU }\end{array}$ & प्राण & 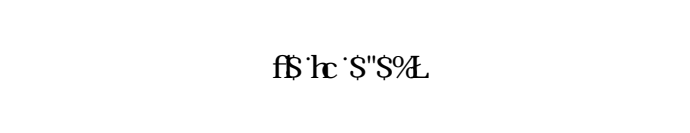 & 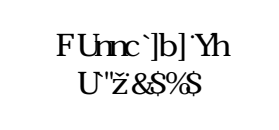 \\
\hline \&DQDGD & $\begin{array}{l}\text { 7UHDMAG } \\
\text { GUQNG } \\
\text { ZDMA }\end{array}$ & 喵 & 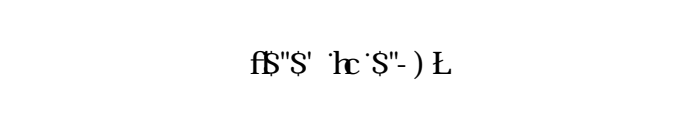 & 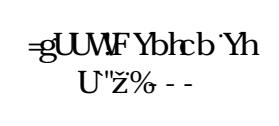 \\
\hline * HP DQ & $\begin{array}{c}\text { 7UHDUAG } \\
\text { GUQNG } \\
\text { ZDMA }\end{array}$ & प्र & 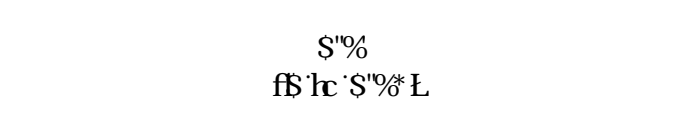 & 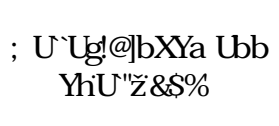 \\
\hline +Xg DU & $\begin{array}{c}\text { 7UHDMAG } \\
\text { GUQNG } \\
\text { ZDMA }\end{array}$ & प्राण & 䛛 & 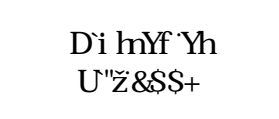 \\
\hline -DSDQ & $\begin{array}{l}\text { ) LQAAHG } \\
\text { ZDMAU }\end{array}$ & प्र & 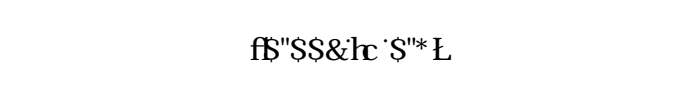 & $\begin{array}{c}\text { +DKKIP RVRLHW } \\
\text { DQm }\end{array}$ \\
\hline $5 \times M D$ & $\begin{array}{l}\text { 7DSZZDMAU } \\
: \text { HQRZDMA }\end{array}$ & 喵 & पाण्ण & 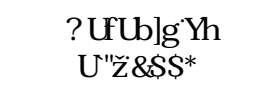 \\
\hline $\begin{array}{l}\text { 6RXVK } \\
\text { \$IUFD }\end{array}$ & $\begin{array}{c}\text { 7UHDAG } \\
\text { GUQNG } \\
\text { ZDUN }\end{array}$ & 喵 & ( & 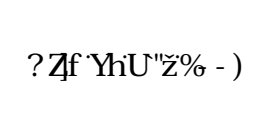 \\
\hline 6SDQ & $\begin{array}{c}\text { 7UHDMAG } \\
\text { GUQNG } \\
\text { ZDMA }\end{array}$ & प्राण & 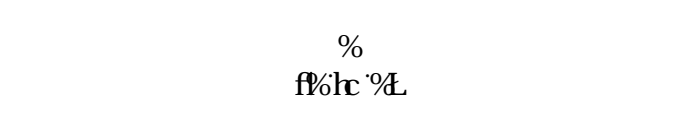 & 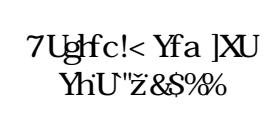 \\
\hline 6SDQ & $\begin{array}{c}\text { 7UHDMAG } \\
\text { GUQNG } \\
\text { ZDUA }\end{array}$ & प्र & 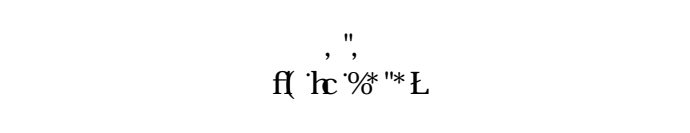 & 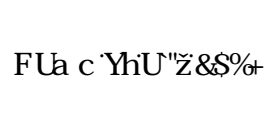 \\
\hline 6SDQ & $\begin{array}{c}\text { 7UDDAG } \\
\text { GUQNG } \\
\text { ZDMA }\end{array}$ & 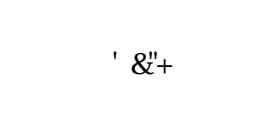 & 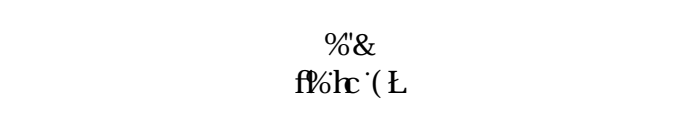 & 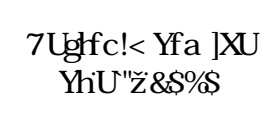 \\
\hline
\end{tabular}




\begin{tabular}{|c|c|c|c|c|}
\hline Country & Sample Type & $\begin{array}{l}\text { Percent Positive } \\
\text { Samples }\end{array}$ & Concentration Average (Range) Oocysts/L & Reference \\
\hline $\begin{array}{l}\text { 8QUAG } \\
. \text { LQ GRP }\end{array}$ & $\begin{array}{c}\text { 7UHDAAG } \\
\text { GUQNG } \\
\text { ZDMAU }\end{array}$ & $\mathrm{am}$ & 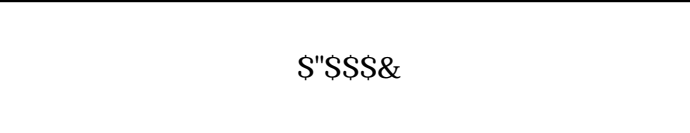 & 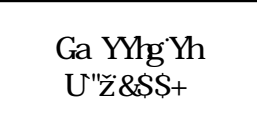 \\
\hline $\begin{array}{l}\text { 8QUAG } \\
. \text { LQ GRP }\end{array}$ & $\begin{array}{c}\text { 7UHDMAG } \\
\text { GUQNG } \\
\text { ZDUAU }\end{array}$ & ( & $\mathrm{am}$ & 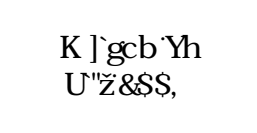 \\
\hline $\begin{array}{l}8 \text { QUAG } \\
6 \text { WVANN }\end{array}$ & $\begin{array}{c}\text { 7UHDMAG } \\
\text { GUQNG } \\
\text { ZDMAU }\end{array}$ & 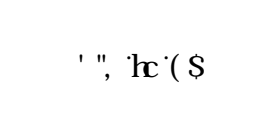 & 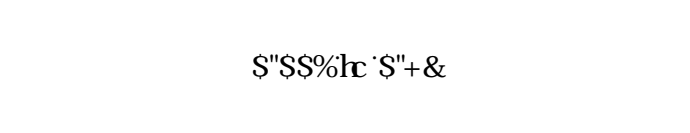 & 5RИ-血口प0 \\
\hline $\begin{array}{l}8 \text { QUAG } \\
6 \text { VIMAN }\end{array}$ & $\begin{array}{c}\text { 7UHDMAG } \\
\text { GUQNG } \\
\text { ZDUAU }\end{array}$ & 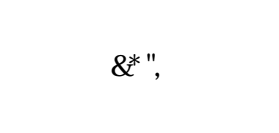 & 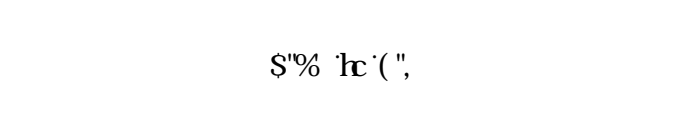 & 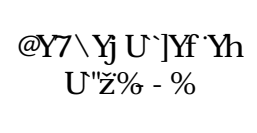 \\
\hline 9HQH $\times \mathrm{HD}$ & $\begin{array}{l}\text { 7UHDMG } \\
\text { GUQNG } \\
\text { ZDMA }\end{array}$ & $\mathrm{Qu}$ & 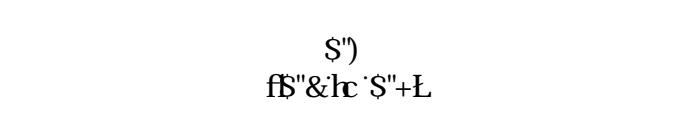 & 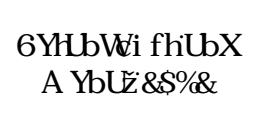 \\
\hline
\end{tabular}

, Q7] DMQ \$P HUFDTRQIRUP DURQRRQMKH/SUHYDOTQFHIDQG GHMFURQR [Z DUALRLQHSDDMUF[SLRVR] RDIVYP RUF[OP LMAG TFRVDGR* DUFLTHMDQI KDYH[GRFXP HQMAGWKKHSLHM-OFF-RICCryptosporidium RRF WW

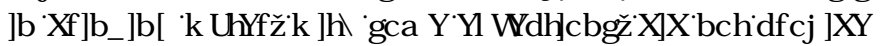

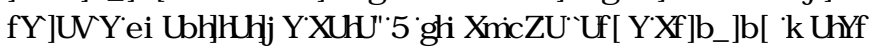

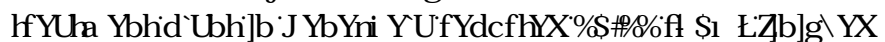
GUQNLO] [Z DMAULMDP SONFRQVDDQHG RRFI WW]ZZ LVK LMKH

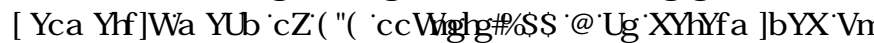
IP P XQRI QRLHMFHQMDWD IP IFLRVFRSI U4 XLQMART/HMDOFRXW

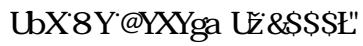

, Q\$\$ ИDWFDSDQTKDVGRFXP HQAAGWXGHMRQT ryptosporidium

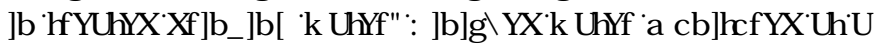
WHDW HQMS DQQMKRZ HGMKDMC ryptosporidium RRF WMZ HUH

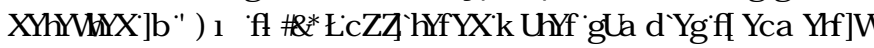

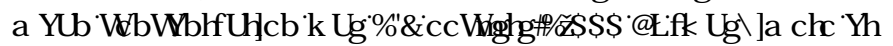

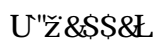

5H XOURU IRUM WAP DMF DDQGZLLHSUHDGP RQLRUQ [RI Cryptosporidium LQHUKHULZ RUTMHDMAGZZ DMAUKDVRQQ

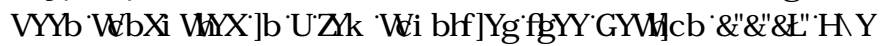
8. GUONLG RDMAUUH XOMRQMLOFQGGUKHP RWROMOVYH

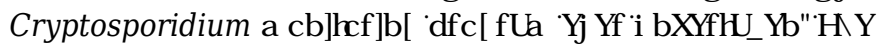
UHJ XФWRQ [UHTXLHG TFRQWQXRXV P RQLVRUL] [RI

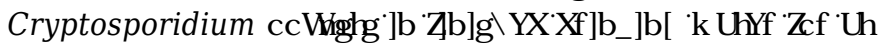

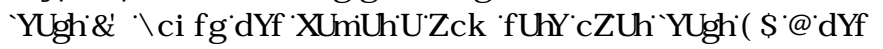

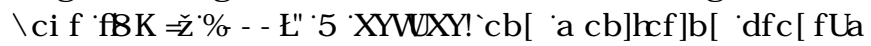

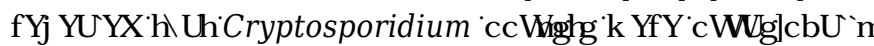

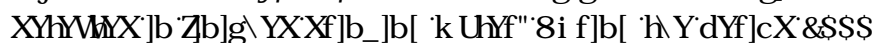

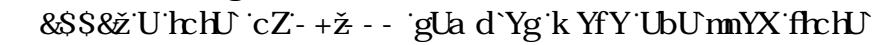
YRQP H

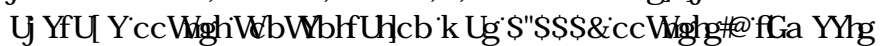
HMLQM \HDW[GHP RQWWDMAGLMH[SUHMHOFH[RI [Cryptosporidium RRF WWLQ

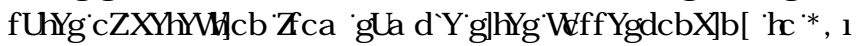

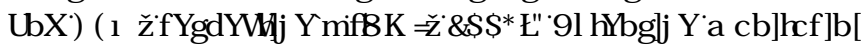

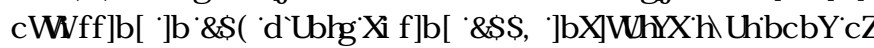

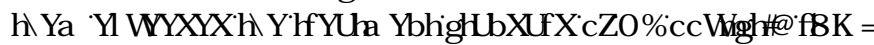

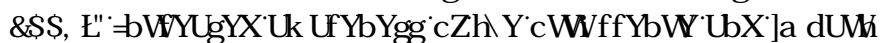

RIGU SURVSRUGXP RRRF WIRQGUQND [ZDMAUP I KVIKDYH

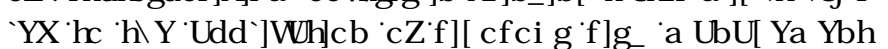

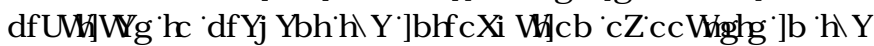
GUQNG [ZDMUUXSSQ]

, Q6FRVOQGIFRQMQXRXVP RQLRUO IRIDLQLKHGZZ DUAUGXUG

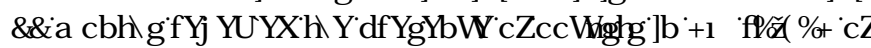

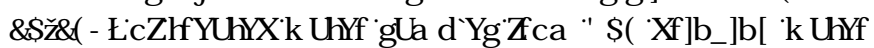
VXSSQHVD TKH P D[ IP XP TFRQFHQWDWRQ IUHSRUHG FRUHSRQGHGYRा]

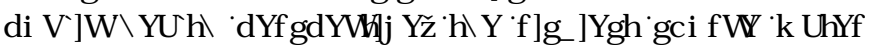

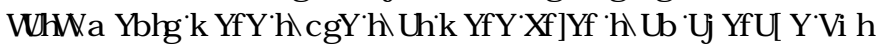

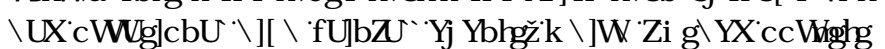
LQRRVMKHZZDMAUT KHDDSSOFDMRQIRI DPP ROFXOUIDSSURDFK

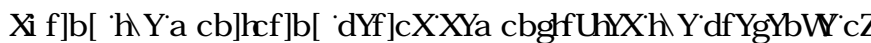
P XOSSOIVSHFLHDDQG] HQRWSHMQGGUIHHONELRSRUROMIC. andersoni WII [FRUHSRQGL] [YRDPFHUYLH] HQRWSHWC.

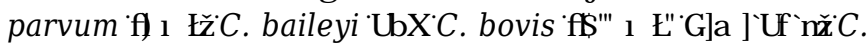

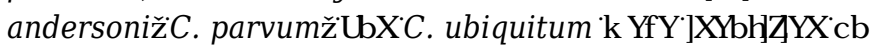

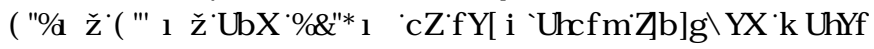

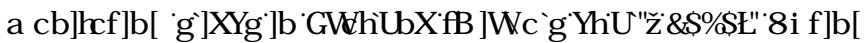

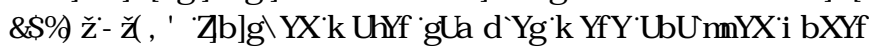

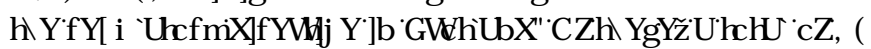

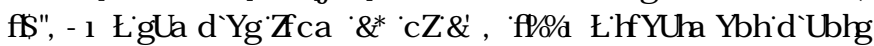

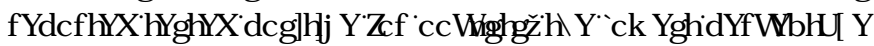

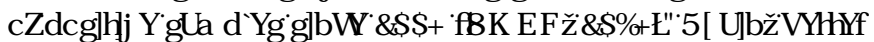
ZDVHUKHGP DQD HP HQWIDQG RSHUDRQDQIP SLRYHP HQW

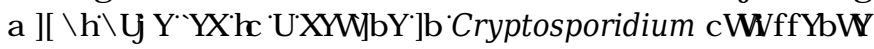
LOMAHGUONG [ZDMUIXSSQT

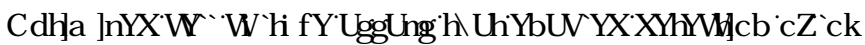
OYYHOVRI RRFI WWZ HUH[XVHG]VRLDWHWLYKH[SUHYDOHOFH[RI

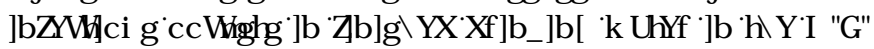

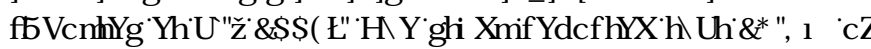

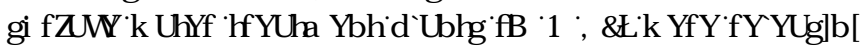

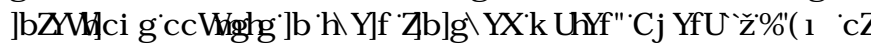

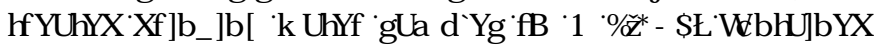
LQ HFWRXVECryptosporidium RRFI WWEXVMQDDCFDVHMLYKH

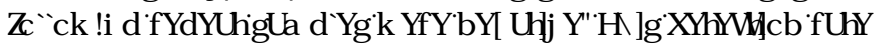

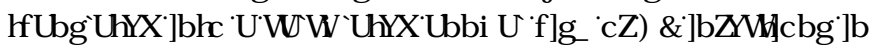

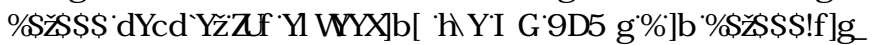

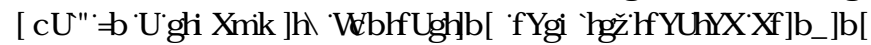




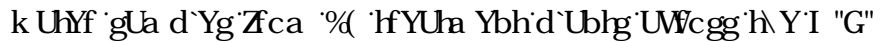

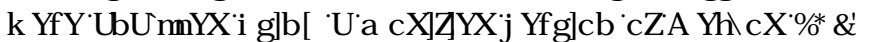

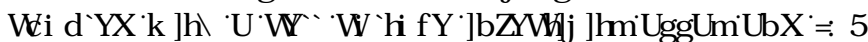

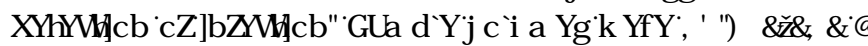

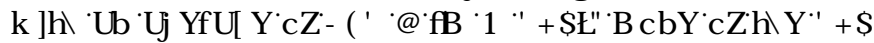

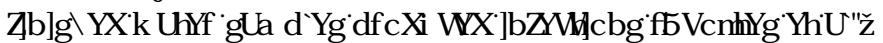

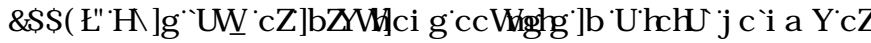

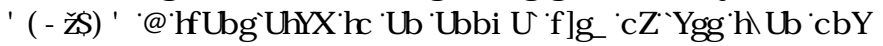

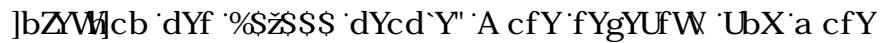

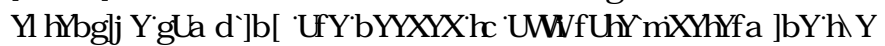
SUHYDOAOFHRRIKXP DQIQI HFURXVRRF WMLQTW-DUAGGUQNG

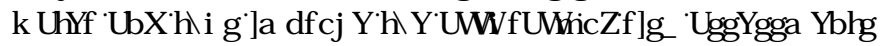

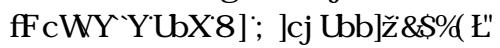

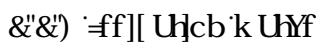

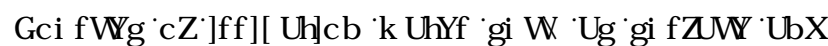
J URXQGZ DMAUTFDQEFHFRP HIFRQVDP LQDMAG ZZ LUK [SDDDMUAN

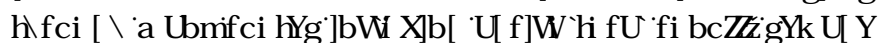

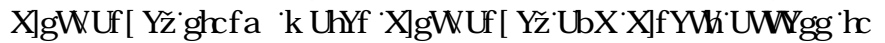

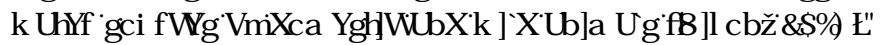
,QDJ UFXOXLDQMHMQ VTIXLMDQGYYH HMEEOMFDQTEHFRP H

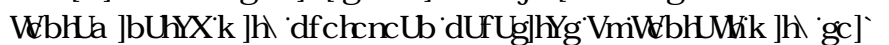

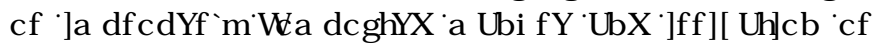

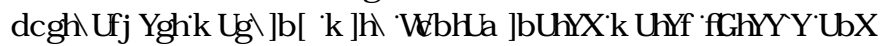

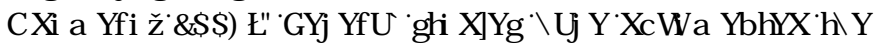

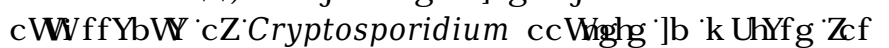

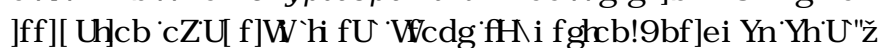

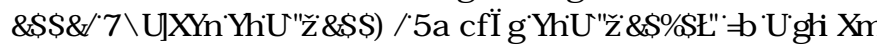
FRQGXFUAGDQ] H IFR ШCryptosporidium RRF WWZ HUHRXQG

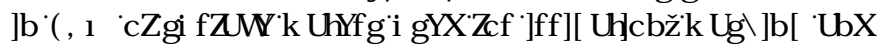
GLVQ HFWOJ DSSSCFDWRQV [ZLIK RRFI WWFRQFHQWDMRQV

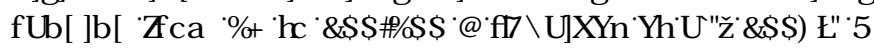

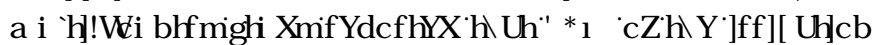

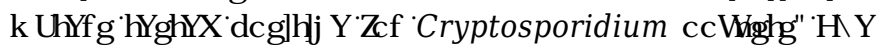
WXG UHSRUAGGUTTXHQMRFFXUHQFHRIRRF/ WMLQTWW DURQ

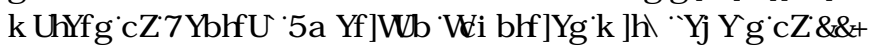

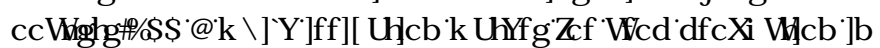

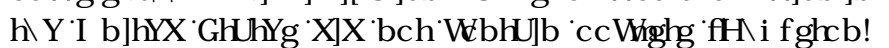
( QUTXH] [HMDQT] Cryptosporidium RRFI WWZZHUH]RXQGLQRZ DMAULMDP SON

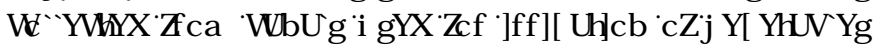
[\$P RLRVHMDDM

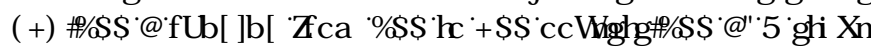

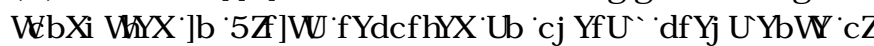

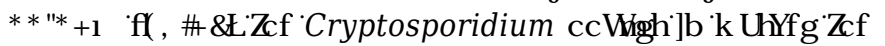

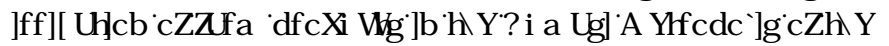

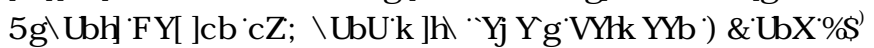

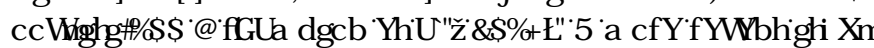

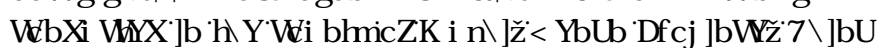
UHSRUAAGFRQFHQWDUROVRI

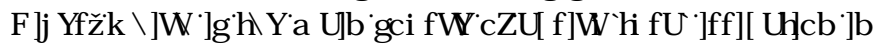

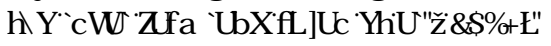

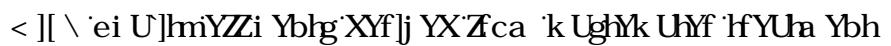

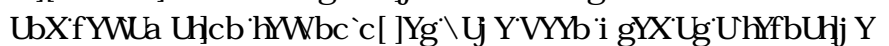
Z DUAULRXUFHMIRUD UFXOXUDODQGDDQGVDSHLW] DURQTR

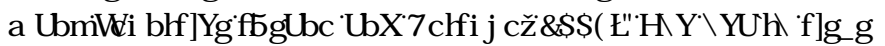

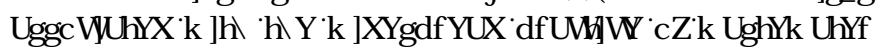

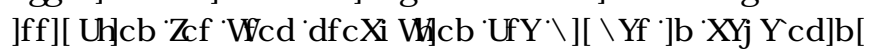

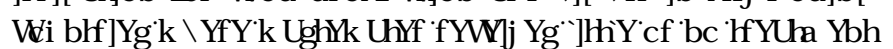
EHRLHXYH
\$ LMXG ]UHSRUAG[MKH[RFFXUHQFHRI [Cryptosporidium

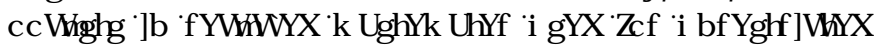

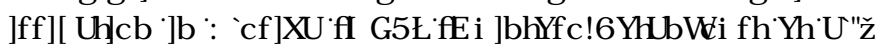

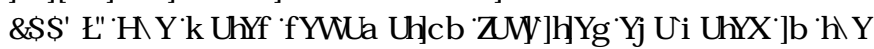
WXG [SLRYLGHG[FRQYHOMRQDQDFWYDUAG[VOGG H[WHDVP HQW

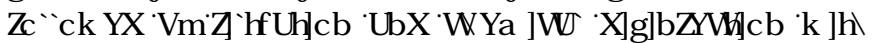

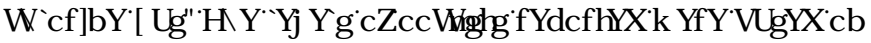

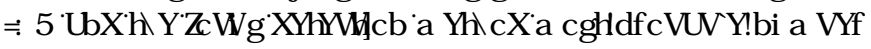

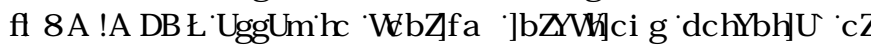

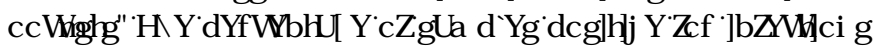
RRF WWZDM UDg HGEHZHHQWDQG

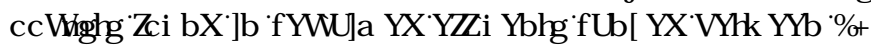

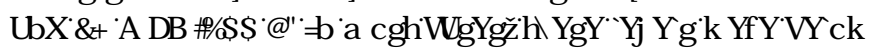

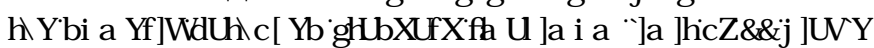

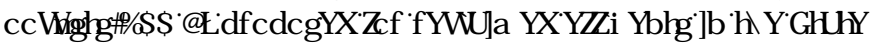

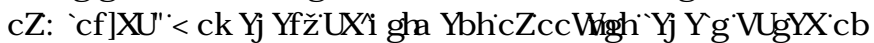
UFFRHU [HI IFLHQF] [GDUDLQGFDUAG]MKDUMKH TFRQFHQWDURQV

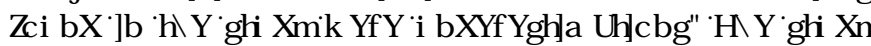

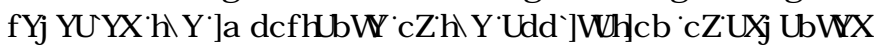

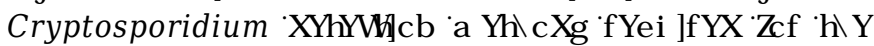

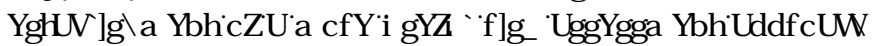
IRU]HFCOIP HG[Z DMAU[DQG[MKH]P SRUDQFH[RI [SDMKR] HQ]

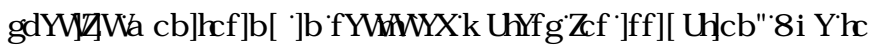
WKH UHFDF LWDQWLQDUXUH RRI RRFI WW [MR [GLVQI HFWRQ

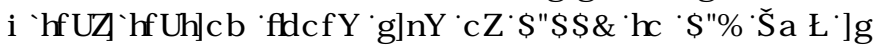
UFFR P HQGHGR RUFRP SOMAIUPP RYDQRI CCryptosporidium

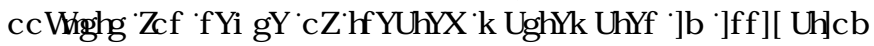

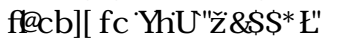

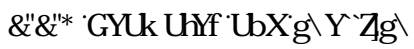

7KHLMDDORZ FFRDWDORFHDQIP SDFUAGEI IKXP DQZZ DUAN DQG [SRQXWRQTCGHQ ]XQRII PP DI IKDUERXULQXP HURXV

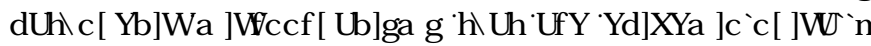

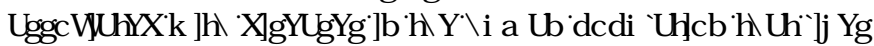
DRQ LMKHFRDWOQH KDYHEH HQGGMAF UAGLOTP DUQHZZDMAUZZ RUCZZ LG TI/DMKLO EHDFKHMLQTHDZ DUIP SDFVAGEI P DUQHLM-ZD HGLLFKDY H

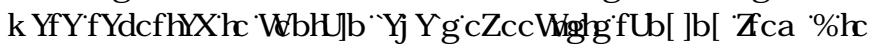

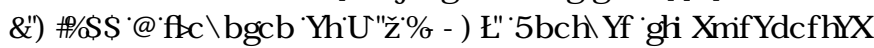
QRZ IFRQFHQWDWRQVIRI RRFI WVIQLMRSLFDQUFFUHDMRQDO P DUQH[Z DUAUVRI [9 HOH] XHOLFRQMDP LQDUAG ZZ LMKLYHZ D] H]

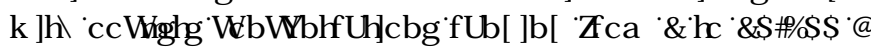
[P/HWDOFRXUMHDDQ

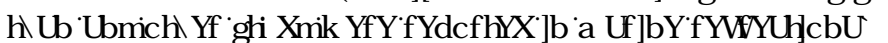

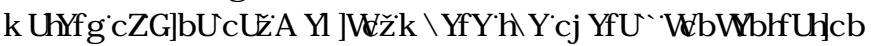
RI RRFI WWJDg HG URP DYHD HRV प्रापा

,QDDGIURQIELYDOHHP RQXVFDQTKHCOLKWIP XWHOIRI WALV

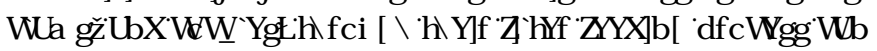

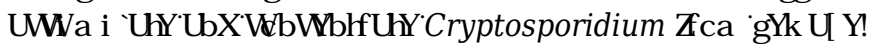

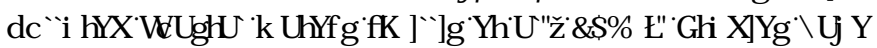

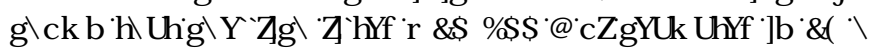
[GHSHQGL] RQIVSHFLHMDDQG[FDQDFFXP XOOMIVHU [KL K

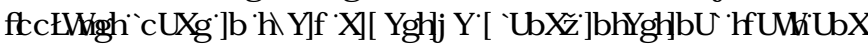

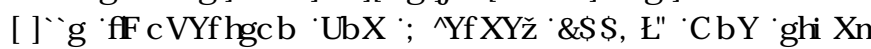

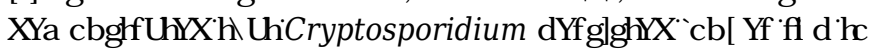

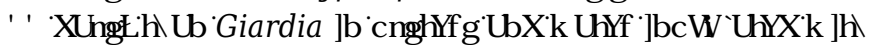

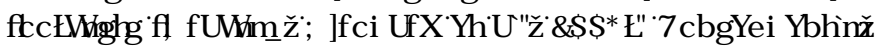




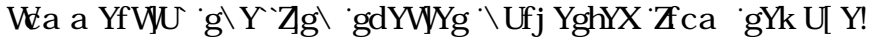
SRQXVAG ZZ DVALVFDQDFWDVIWDQVP LWRQ GYHKLFON RI

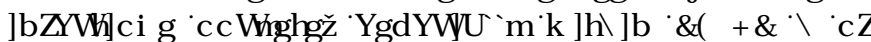
FRQVDP LQDWRQ DVV [GHP RQWWDVAG QQ IUHFHQWIMXGLH

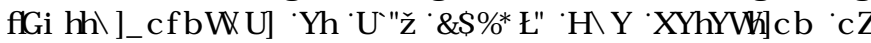
Cryptosporidium DQG[RUKHUSLRVR] RDQ[SDUDMUAVIXFK[DV Giardia DQG[Toxoplasma LQMKHCDLK[GHMQHG]RUKXX DQ

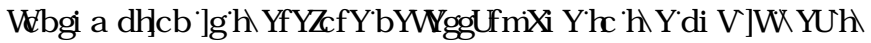

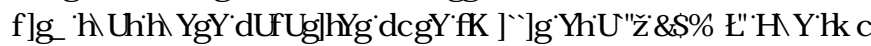
P DQRSSHFLMRICryptosporidium UHSRUAGLQRI WAUDUHC.

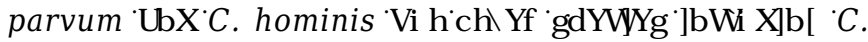

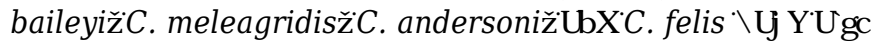

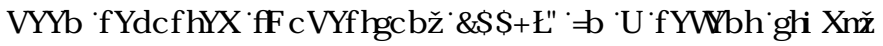
Cryptosporidium RRF WWZ HULLROMAGIURP IRXUUSHFLMRI

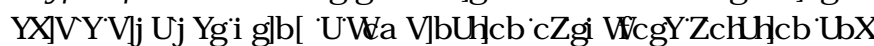

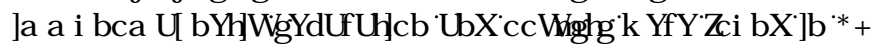

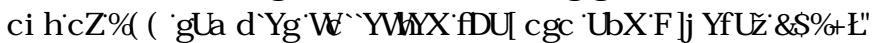
' 1 \$[VHTXHQFHDQDQ VVRR [MKH SUHMHFHRR $\square$. parvum $\square$ C. hominis $\square$ DQG $[C$. meleagridis $\square$ 6WXGHMKDYHDOREHQTRQGXFAGURLGHQW IVXEWSHMRIC. parvum DQG[C. hominis [XVQ] [MKH $10[N$ ' D] QFRSURVAL

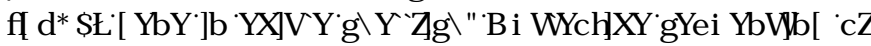

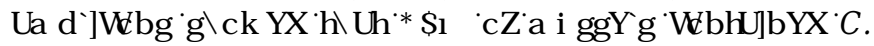

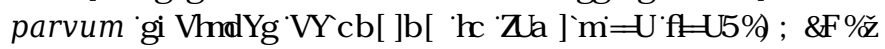

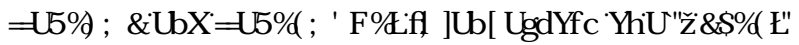

7KH\$SUHMQFHRR IQ HFWRXVCryptosporidium RRF WWZDV

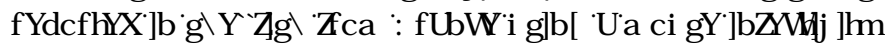

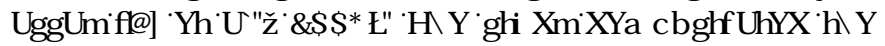
SUHMQFHR LRRF WLQDDQMDP SOMIRUDQMUAMDQGMHDRQV DQGDOMK Z DVIKKHP RWVFRQNDP LQDUAG SDUMT KHLDMARI GHMAFURQZZDVIDSSDUHQVQ [UHODVAG ZZ LVK LMHDVRQDOUDL

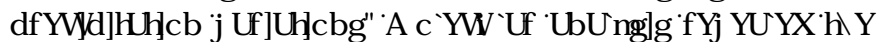
SUHGRP LQDQFHRIC. parvUm [ONHQFDWOEUHGL [RU, LQRQ

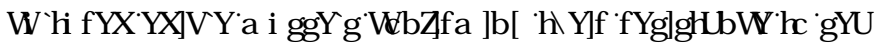

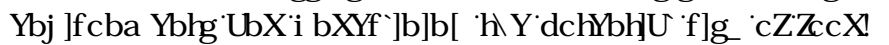
ERLOHLQ HFRQ]

\&RP P HFIDQDOGQRQTRP P HFLDQRI WALVDQGRI WATFXOXUH

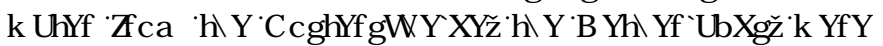
H DP QHG]RUUKHSUHMQFHR [Cryptosporidium RRF WWDQG

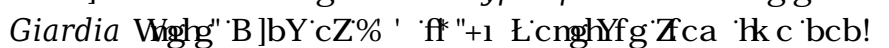
FRP P HFIDOKDUYHMQ [MUAVFRQVDQHG Cryptosporidium? Giardia RUERИKए6L RI

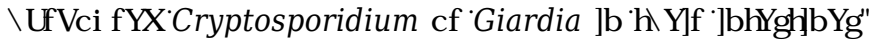

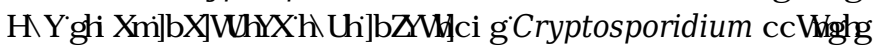

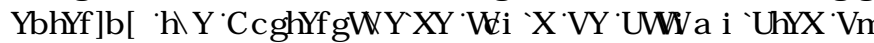
RI WALV[GHMQHG]RUTKXP DQ[FRQVXP SWRQ [6FKHWLHWDQ] प्रणपा

C. parvum RRFI WZZHULDQRUFRYHHGIUR FEUDP XWHQ

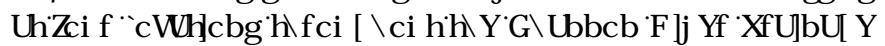

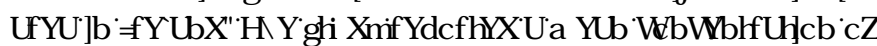

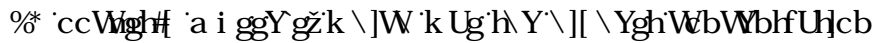

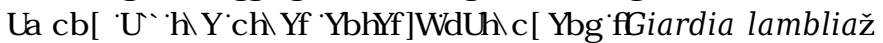
Encephalitozoon intestinalis $\mathbb{E}$. hellem $\mathbb{D D Q G E n t e r o c y t o z o o n}$

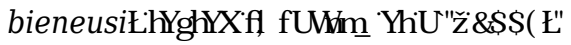

7KH[SUHMHFHR [Cryptosporidium LQELYDOH[KHCDLKCKDV DQREHQGGP RQWDUALQRUKHU XURSHDQ8 QRQTRXQNHN

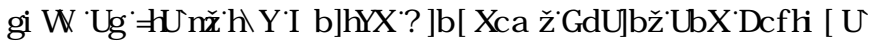

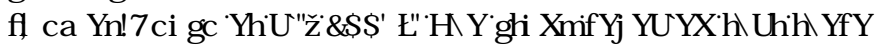

ZDVORUHOWRQEHE HQLMYSUHMHOFHRI Cryptosporidium RRF WMDQGMKHP IFURELRQI IFDQFRQMPP LQDRQGHMFWGLQ

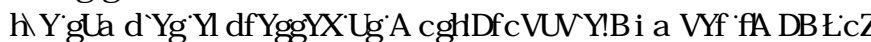
IHFDOFRQI RUP VIR QHIP SRUNDQWI LQGLQ] [Z DVIMKDWIMKH GHSXUDRQ[SURFHW[ZDVIQH IHFWYH[Q]VRVDC [UHP RYL] RRFI WVFRQMPP LQDMRQTO RUHRYHUTMKHIWXG IUHYDOGLYKH H LWAQFHRIYLEOIRRF WLQTMP SOMZLLKL IFURELRQI IFDO FRQNDP LQDURQQPYHQ/[RZ HUYKDQ

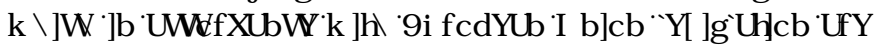
FRQMGHUHGLXLVEOCIRRUKXP DQFRQVXP SWRQTVKH[WXG

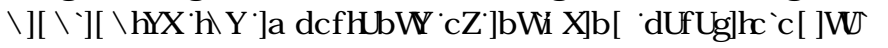

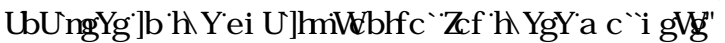

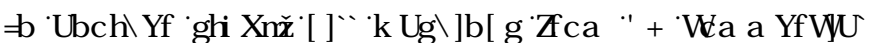

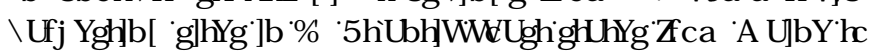

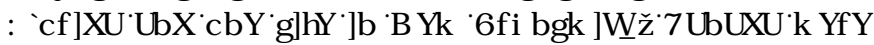
H[ DP LQHG IRU [Cryptosporidium RRF WW EEI

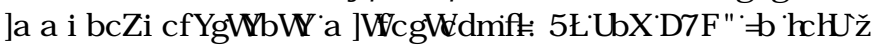

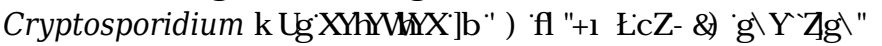

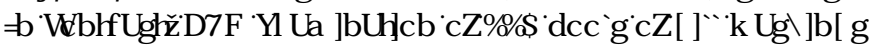
IURP [RI WHUV [DQG DI [SRRQ TURP [FOP V [GHVHFVHG

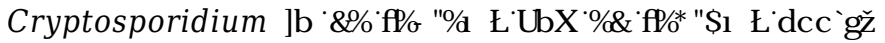

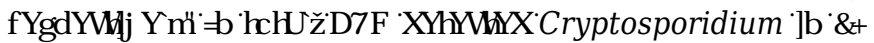

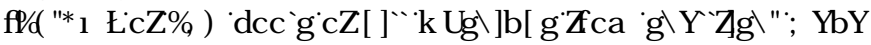
ИHTXHQFL] [RI [B\&5 [SURGXFWLGHQW LGGCryptosporidium

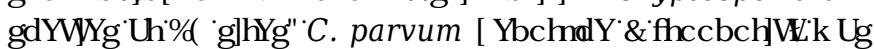

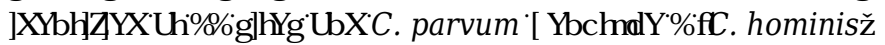

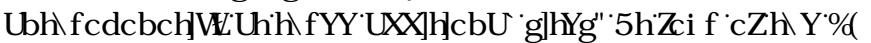

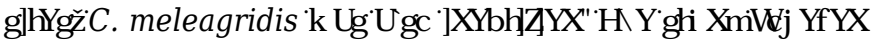
VKH [ODU HWV HRJ UDSKLF DDUHDRI DDQI DVXYH QRU Cryptosporidium DQG LQGLDVHG [ZLGHSUHDG DHFDO FRQMDP LQDMRQ]URP [KXP DQIDQG[SRWEOAIDQIP DQMRXIFHN १ D HYMDQ⿴囗⿰丿㇄口⿴囗十

6LQFHEELYDOHLMHCLLK FDQR I IFLHQVQ FFRQFHQWDMADDQG UHMQTHOYLRQP HQMED GHUYHGSDMRI HQMTRU[RQ] [SHURGY]

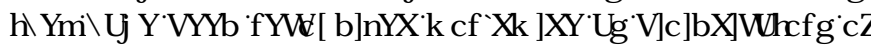
DTXDWF THYLLRQP HQW[ZLKTHFDQRU LQRU DQLP VD RU LQMDQFHDMHQMQHQFCDP [RXVBCQMQ] [XVHGLYRDWHW[पKH GWUEXVRQDQGP D QLXGHRIIFDQFRQMPP LQDWRQTQJMLUH UYHUQHK WAP VLQEDORLQDGHP RQWDMAGWMHSUHMQFHR

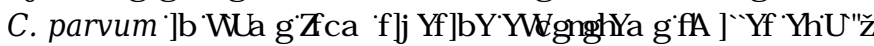

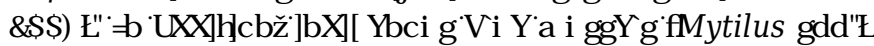
XVHGDVIELRVHQVQHQVIR P RQLVRU RUDMKH SUHMHQFHRI

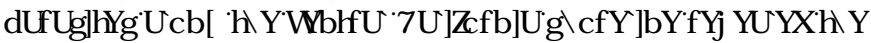
SUHMQFHRR [Cryptosporidium DQG [Toxoplasma gondii LQ

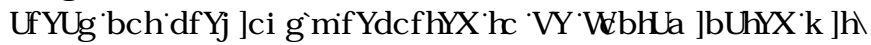

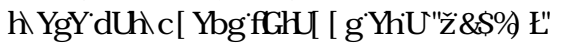

\subsection{Persistence}

DUDRQYMKHSHUMWAOFHRICryptosporidium RRF। WLQ

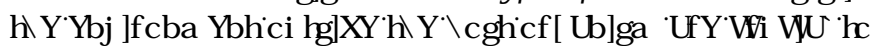

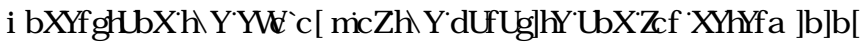
ИKH UMN WDQVP LWRQRRI [Cryptosporidium LV] RYHUHG[E] [MKH

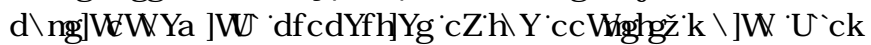

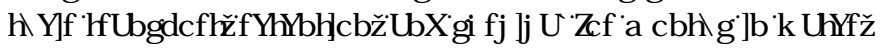
VRLQIYH HDEOMMDDQGP RQXVMZ KIFKDDHP DKRUUHMUYRLV

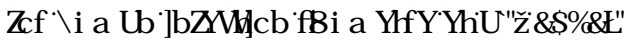

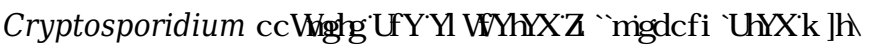




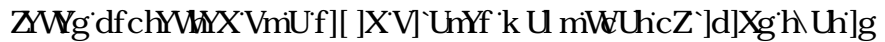

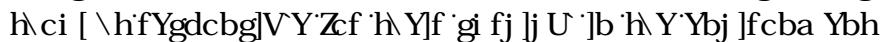
DQG]RU[MKHLU[WDQMVVIKLRX] K[MKHLMRP DFK[DQGLVP DO

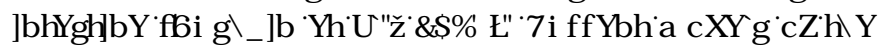
RRFI WQVIXUDFH[FKHP LWM [VX] J HWVMKDVMKKHFKDY HIDQG KI GURSKRELF [FKDUDFUAULMFVIRI [MKH[SDUDMUA[VXU DFHLFDQ J HQHUDMALDQG P RGXOUACHOFFRWDWF DDWDFWYHLDQGRU

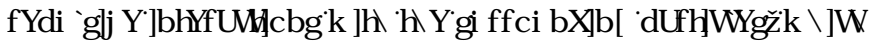
FUWFDQ [DI IFVVMKH[EHKDYLRURI [MKLV[SDUKR] HQ[DQG]W

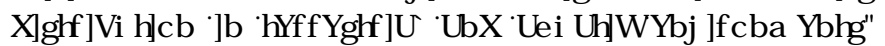

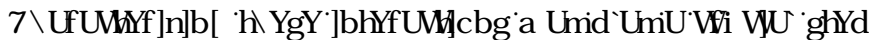
IRUPP DQD LO] [KKH[HQYLRQP HQVDOP DWFHNDWULVIRI

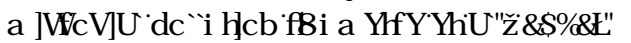

7KH[SHUMMAOFH[R] [Cryptosporidium RRF WTRQ[ИALHMUDO DQGDT XDMF [HOYLRQP HQWLMLQ $Q$ XHQFHGE I [MKHFRP ELQDMRQ

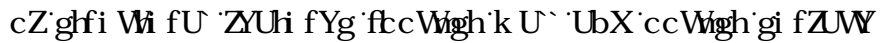

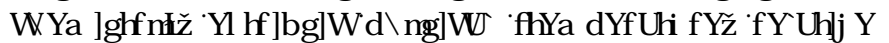
KXP LGLWGMMFFDMRQTXONDYROMMDGLDRQIDQG[FKHP LFDO

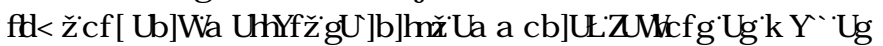

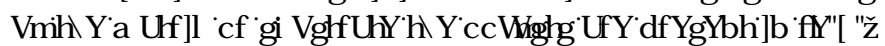

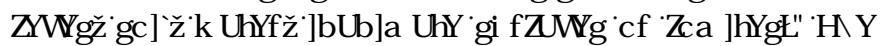
ELRR] LFDORPVD] RQLVP [DQG [SRVAQVDOLSUHGDVRQ RI

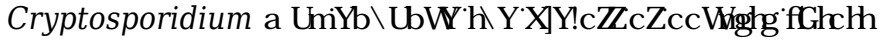

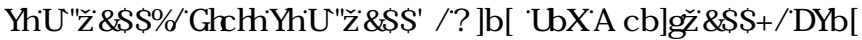

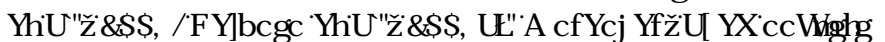

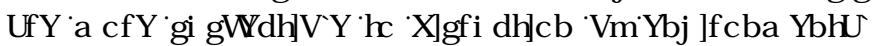

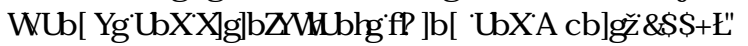

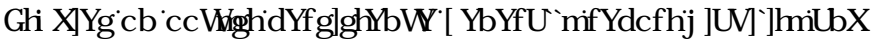

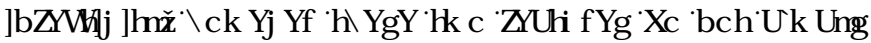

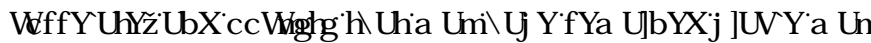

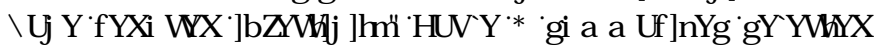

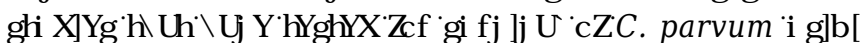

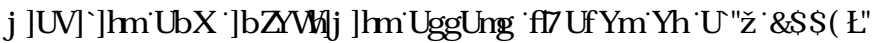

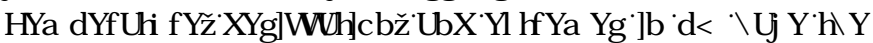
P RWVGHWUP HQVDOTH I HFWRRQ RRFI WVIXUYLYDOTQ [MKH HOYLRQP HQMTHFHQNLQMHMDQm HMDQm

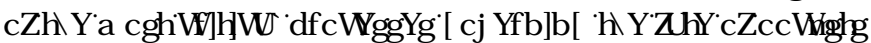

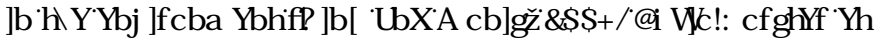
DQMسل]

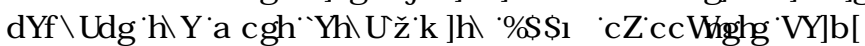

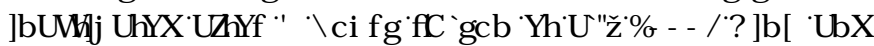
0 RQVMा

Table 6. Environmental factors influencing the viability and infectivity of $C$. parvum oocysts (Carey et al., 2004)

\begin{tabular}{|c|c|c|c|c|c|}
\hline $\begin{array}{c}\text { Environmental } \\
\text { Media }\end{array}$ & $\begin{array}{c}\text { Temperature } \\
\left({ }^{\circ} \mathrm{C}\right)\end{array}$ & $\begin{array}{l}\text { Time } \\
\text { Days } \\
\text { (Unless } \\
\text { Otherwise } \\
\text { Noted) }\end{array}$ & $\begin{array}{c}\text { Reduction in } \\
\text { Oocyst Viability/Infectivity }\end{array}$ & Assessment Method & References \\
\hline $\begin{array}{l}\text { / DERLDMRU } \\
\text { : DUAU }\end{array}$ & 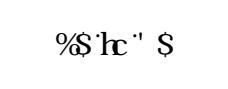 & प्र & 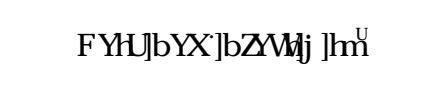 & $\begin{array}{l}\text { \% / \%FP RXYH } \\
\text { LQ HFUYW }\end{array}$ & 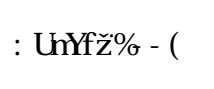 \\
\hline $\begin{array}{l}\text { / DERLDMRU } \\
\text { : DMAU }\end{array}$ & प्राण & 口P LXUN & 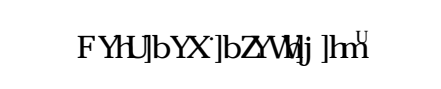 & $\begin{array}{l}\text { \% / \%FP RXVH } \\
\text { LQ HWULW }\end{array}$ & 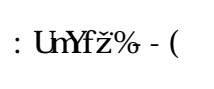 \\
\hline $\begin{array}{l}\text { / DERLDMRU } \\
\text { : DUAU }\end{array}$ & प्रा & DPP LXXN & 1 RQRQ IFWRXV & $\begin{array}{l}\text { \% } \$ \text { \%FP RXVH } \\
\text { LQ HFUYLW }\end{array}$ & 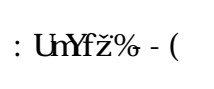 \\
\hline $\begin{array}{l}\text { / DERLDMRU } \\
\text { : DUAU }\end{array}$ & ! प्र & 口P LXИA & 1 RQRQ HFWRXV & $\begin{array}{l}\text { \% / \%FP RXVH } \\
\text { LQ HFULW }\end{array}$ & 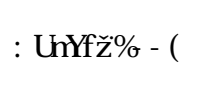 \\
\hline $\begin{array}{l}\text { / DERLDMRU } \\
\text { : DUAU }\end{array}$ & 四 & 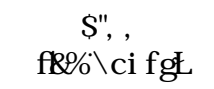 & 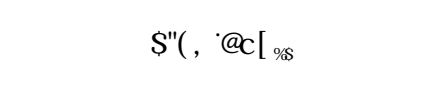 & ' $\$ 3,[B$, & $\begin{array}{l}\text { 5REHURQH } \\
\text { DQm }\end{array}$ \\
\hline $\begin{array}{l}\text { / DERLDMRU } \\
\text { : DUAU }\end{array}$ & 四 & om & ! 四四 $\mathrm{R}_{\mathrm{\square}}$ & ' $\$ 3,[B$, & $\begin{array}{l}\text { 5REHWRQTHN } \\
\text { DQm }\end{array}$ \\
\hline $\begin{array}{l}\text { / DERLDMRU } \\
\text { : DUAU }\end{array}$ & 15 & 6QDSワUH+ாடி & 1 RQIQ IFWRXV & ' $\$ 3,[B$ & $\begin{array}{l}\text { 5REHURQH } \\
\text { DQm }\end{array}$ \\
\hline $\begin{array}{l}2 \text { QVXXUDFHN } \\
\text { ' HMFFDMRQ }\end{array}$ & URRP [MPP S & GKRXXV & ! 喵 $\mathrm{RJ}_{\square}$ & ' $\$ 3,[B$ & 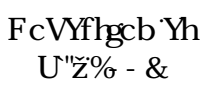 \\
\hline 6UAUGRDMA & 四 & ! प्र & $\begin{array}{l}\text { 5HMQHGYLELQNDQG } \\
\text { LQ HUWLW }\end{array}$ & $\begin{array}{l}\text { 3, H FOMRQDDG } \\
\text { P RXV-RQ IFUYIW }\end{array}$ & 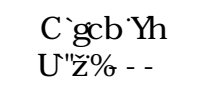 \\
\hline $\begin{array}{l}\text { 7DS[ZDUAN } \\
\text { DEDQRZD } \\
\text { WRLRX] K\K WAP }\end{array}$ & URRP [MPP S & 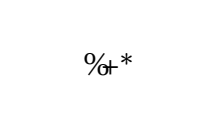 & प्याप R $\mathrm{R}_{\square}$ & ' $\$ 3,[B$ & 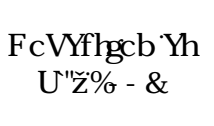 \\
\hline 5 YHYZDMA & DP ELHQMUAP S & 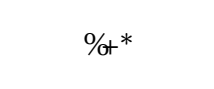 & 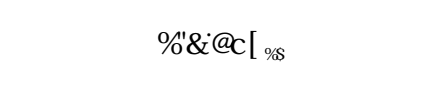 & ' $\$ 3,[B$, & 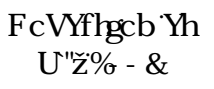 \\
\hline 5 YHUZDMA & 四 & 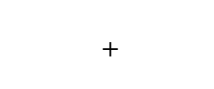 & 1 RQLQ IFURXV & \&HQFXOXUҢ四 ' 0 & 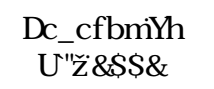 \\
\hline 5 IYHUZDMAN & 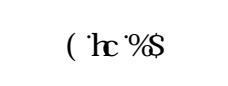 & प्र & 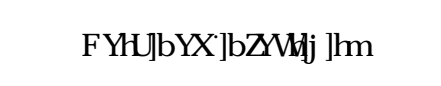 & \&HQFXOXUH四 ' 0 & 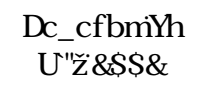 \\
\hline
\end{tabular}




\begin{tabular}{|c|c|c|c|c|c|}
\hline $\begin{array}{c}\text { Environmental } \\
\text { Media }\end{array}$ & $\begin{array}{l}\text { Temperature } \\
\left({ }^{\circ} \mathrm{C}\right)\end{array}$ & $\begin{array}{c}\text { Time } \\
\text { Days } \\
\text { (Unless } \\
\text { Otherwise } \\
\text { Noted) } \\
\end{array}$ & $\begin{array}{c}\text { Reduction in } \\
\text { Oocyst Viability/Infectivity }\end{array}$ & Assessment Method & References \\
\hline 5 SYHZZDAA & 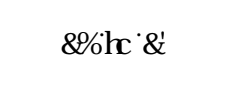 & 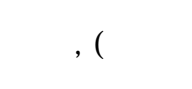 & ! 凅 $\mathrm{R}_{\mathrm{a}}$ & 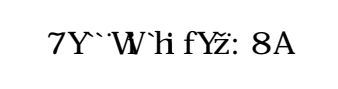 & $\begin{array}{l}\text { 3RNRLQTHN } \\
\text { DQm }\end{array}$ \\
\hline $\begin{array}{l}\text { 6HDZDUAN } \\
\text { WDMF[DE } \\
\text { FRQGURQV }\end{array}$ & Q & प्र & 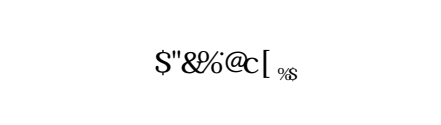 & ' $\$ 3,[B$, & $\begin{array}{l}\text { 5REHUNRQH } \\
\text { DQ }\end{array}$ \\
\hline 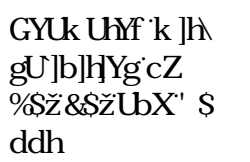 & प्र & प्र & 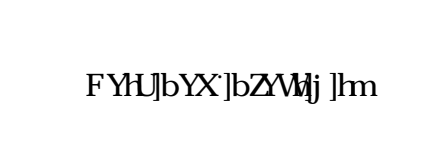 & $\begin{array}{l}\text { \% / \%FP RXVH } \\
\text { LQ HFULLW }\end{array}$ & 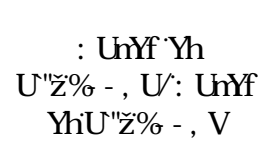 \\
\hline 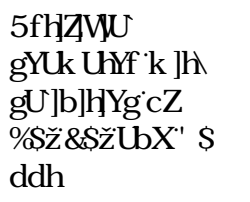 & प्र & प्र & 5HMQ & $\begin{array}{l}\text { \%\$/ \%FP RXYH } \\
\text { LQ HWUYLW }\end{array}$ & 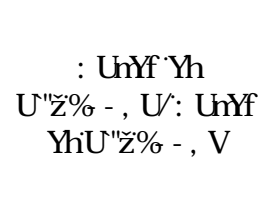 \\
\hline 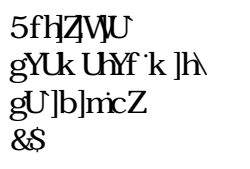 & प्र & $\mathrm{Qu}$ & 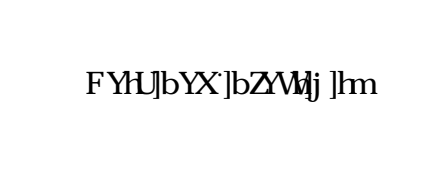 & $\begin{array}{l}\text { \% / \%FP RXYH } \\
\text { LQ HFUYW }\end{array}$ & 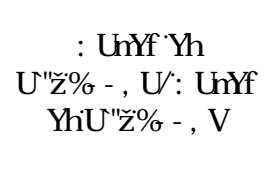 \\
\hline $\begin{array}{l}\text { \$UWLFLO } \\
\text { YHDZDUAZLIK } \\
\text { VDOQLWRIMI } \\
\text { SSW }\end{array}$ & $\mathrm{QP}$ & प्र & 5 HМQ & $\begin{array}{l}\text { \% / \%FP RXYH } \\
\text { LQ HFULW }\end{array}$ & 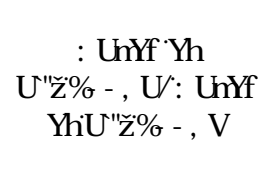 \\
\hline 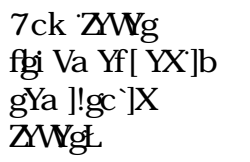 & \$P ELHQVVHP S & 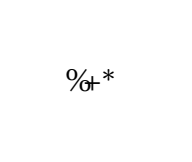 & 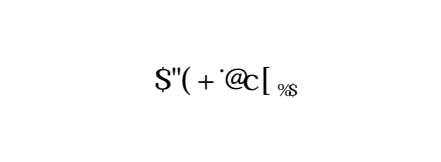 & ' $\$ 3,[B$, & $\begin{array}{l}\text { 5REHUNRQH } \\
\text { DQ }\end{array}$ \\
\hline +XP DQIIFHV & ( & 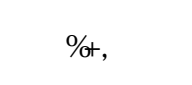 & 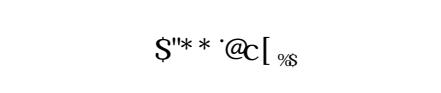 & ' $\$ 3,[B$ & 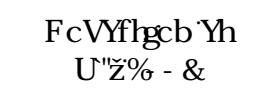 \\
\hline
\end{tabular}

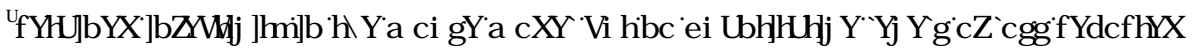

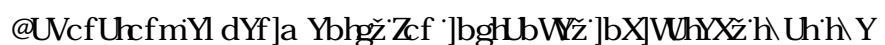

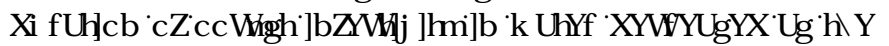

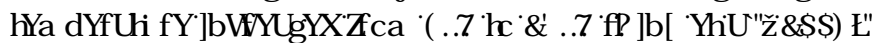

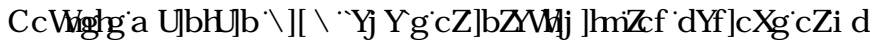

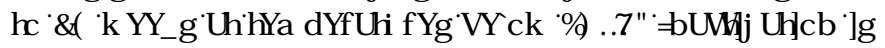

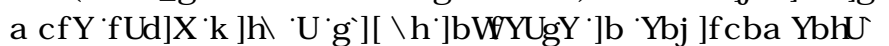

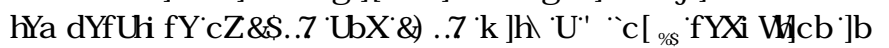

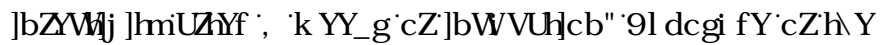
RRF WWLRR

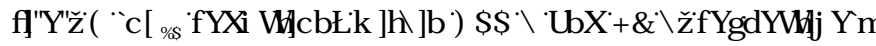

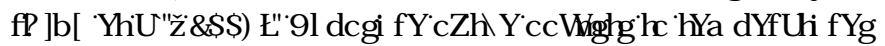
RI

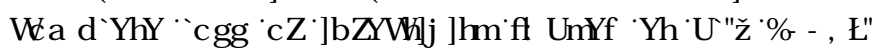
7HP SHUDXUMEHGRZDUH' LQ [DHDORGGMP HQWEQMRIRRF WW

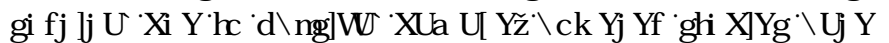

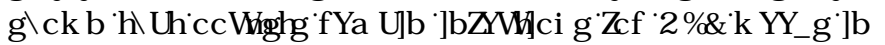

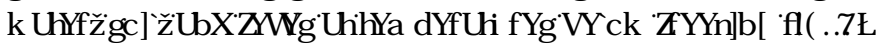

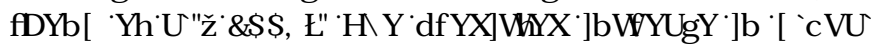
UAP SHDUXUHP D IKDYH[GLP DWF FRQM-TXXQFHMIRURRFI WW

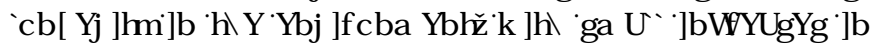

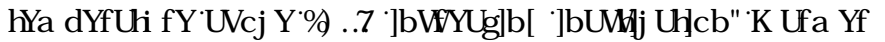

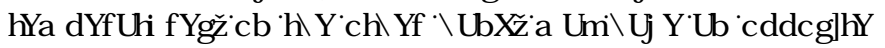

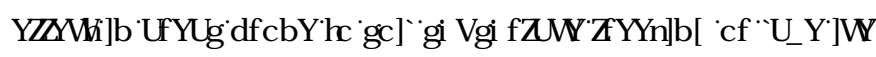
FRYHUIZ KHUHIXEWDOWDQQXP EHUVRIRRFI WMP D UUP DQ

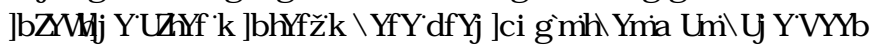

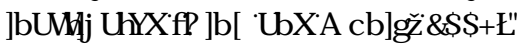

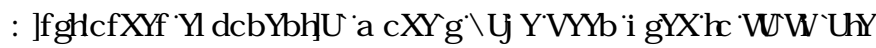
GHRIILDMAVRICCryptosporidium RRF WMLQRZDMAUIVLQIDQG

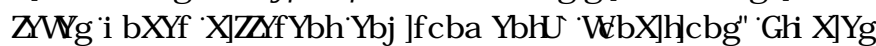
KDYH[MKRZ Q[MKDVMRUD] LYHQ[SHUFHQVDD HRI GQDFWYDUHG

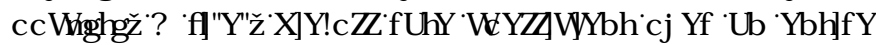
LQFXEDMRQ [SHURG]LVIOYHUVA [SURSRUMRQDOTMR [MKH

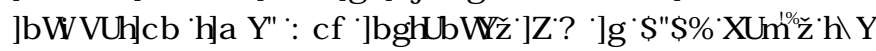

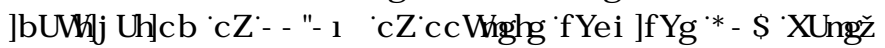
FRP SDUHGJ/Rس

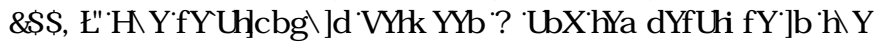

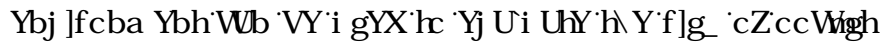
FRQMPP LQDMRQIRUSXE@FIKHDOKX]

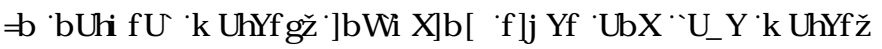

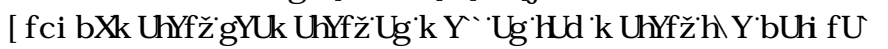
GHRIIRICCryptosporidium RRF WWIVIONHQDVREEHDIIFUAGEI DFRP ELQDMRQLRI DELRWF DQG[ELRMF [MHMHMT KXVTMKH FRP ELQHGLIIFWRRIFKHP LFDQISKI UFDQIDQGEIRQI IFDQZDDAN 


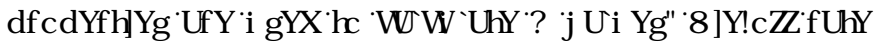

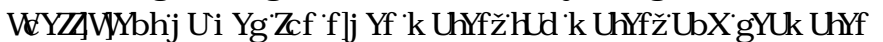
VDNHQGULFV QURP [SXEQVKHGGGDDIRGIFDMA[MKDVVRYHUD UDV HRI 7KHP HDQNS [MDQGDGGGMIDMRQVRI] [YDOXHMUHSRUAG]RU

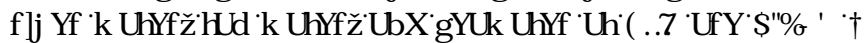

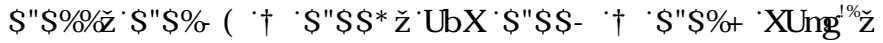
UHSHFUYHQ

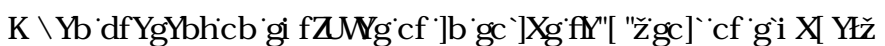

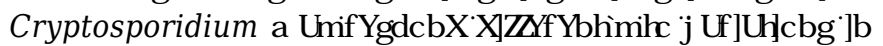

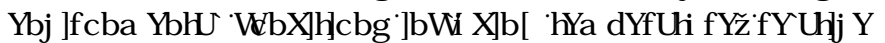

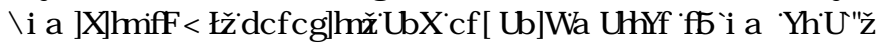

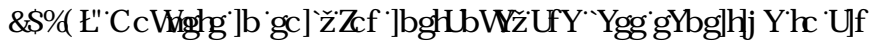
VHP SHUDVXUH DQG IVROUIDGLVWRQ EHFDXVHRRI [MKHLU

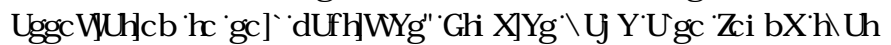

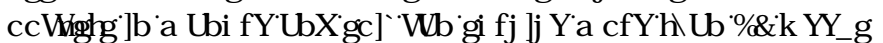

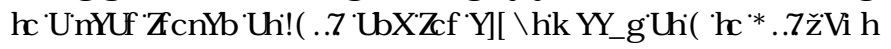
RQQ GHMALP LQHG] RU[GUI HUHQVIRLQ/SUHMHQVDDZ LGH[DOJ HRI

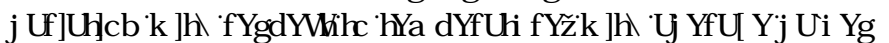
RI UHNSHFUYHQ ШBHQ [HMDQ

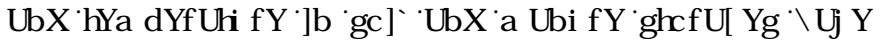

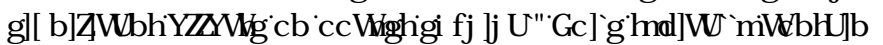

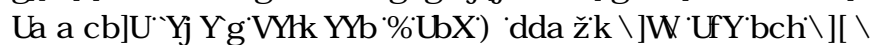
HQRXJ K[YRDDIIFURRF WWTHRZHYHU⿴UHKQ DHURO] HGIVLQV

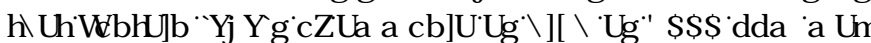
ODGMRLQFUHDHGLDMARIRODFUYDMRQ LQGXFHGLQDFUYDURQIYKXDMRFFXUVGXUG [MRLD HRI DQPP DO

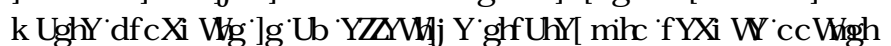
QXP EHULQTOYHMRFNZDUAMEH RUHEHQ] [VSUHDGRQVR[YKH

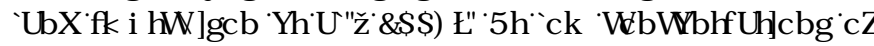

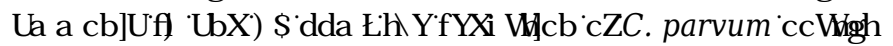
VXUYYLQIYP RUHSLRQRXQFHGRYHUSLRRQ HGSHURGMRILYP H [XS[YRR GHMAUP LQHG EEI [MKH[GUIHUHQWDOTXSVDNH RI $] \$ 3, \mathrm{~B}$,

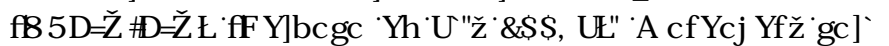

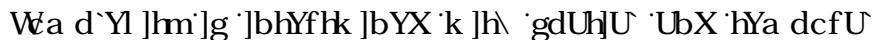
YDUDELOW P RUHLMKDQRZ DVHULDQG RRFI WW[QRLRLQ/DUH

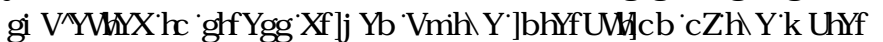

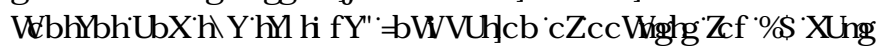

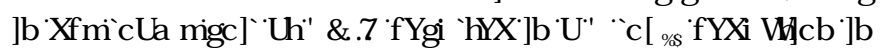

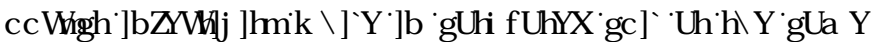

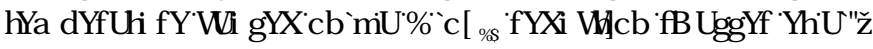

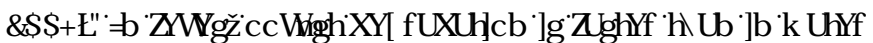
ZLLKLDP P RQLDDQGIFDQRY DQLVP VDVZZHQDDVYAP SHUXXUH SQI LQ [DQIIP SRUDQNGRODDLQDFUYDURQD] HQWRIRRFI WW [BHO [MDDQ

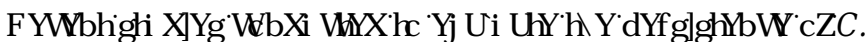

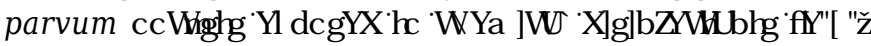

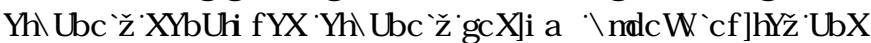

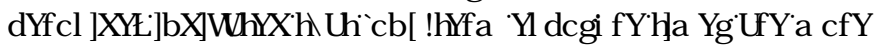

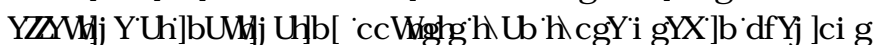

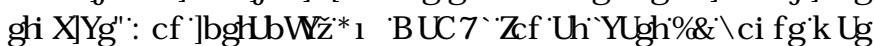

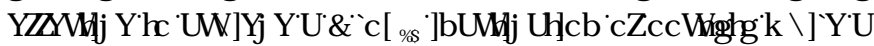
SRRUDHI HFWM

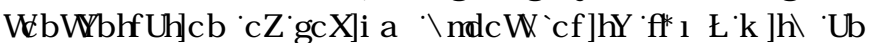
H SRVXUHLP HRI

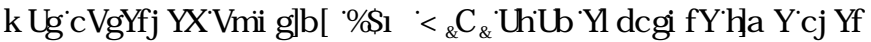

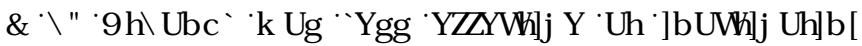
Cryptosporidium RRF WW

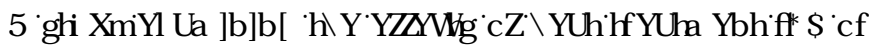

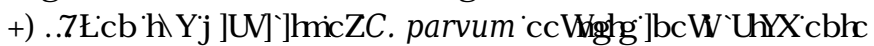

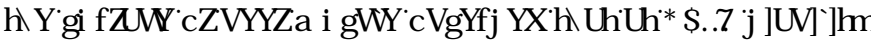

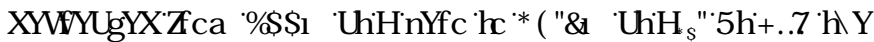

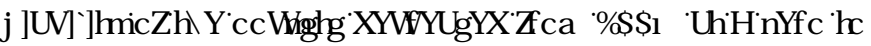

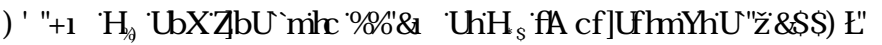

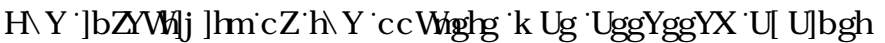

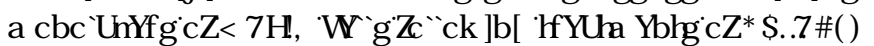
VFRQGVDQG

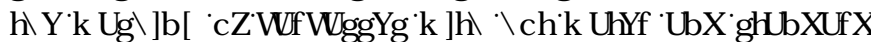

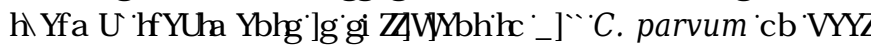
P XVFOO

\subsection{Reduction by Sanitation Management}

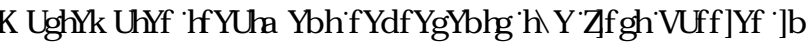

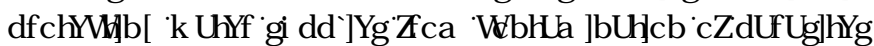

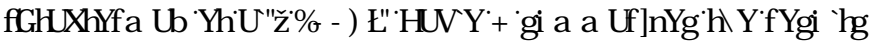

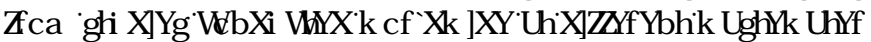

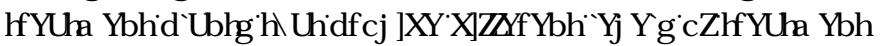
IRUUHP RYDQQDFWYYDURQRI [Cryptosporidium ILRP [M-ZD H] 
Table 7. A Summary of studies on the removal efficiency of Cryptosporidium oocysts by different wastewater treatment processes

$\begin{array}{ccccc}\text { Country Plant Population } & \text { Primary } & \text { Secondary } & \text { Tertiary } \\ \text { Treatment } & \text { Dreatment } & \text { Dreatment } & \text { Disinfection Oocyst/L Oocyst/L } & \text { Log10 } \\ \text { Influent } & \text { Reffluent } & \text { Removerence }\end{array}$ Average $\begin{aligned} & \text { Effluent } \\ & \text { Average }\end{aligned}$

\begin{tabular}{|c|c|c|c|c|c|c|c|c|c|c|}
\hline \%D] & $\square$ & 15 & $\begin{array}{l}\text { 6FU+HQ } \\
\text { DHWMRQ }\end{array}$ & $\begin{array}{c}\text { \$FUYDUAG } \\
\text { VOXG HW } \\
\text { secondary } \\
\text { FOUULFDARQ }\end{array}$ & $1 \mathrm{RQH}$ & 89 & 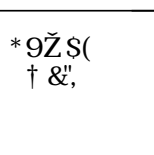 & $2 E+02$ & 䛛 & 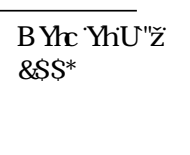 \\
\hline \%D! & $\square$ & 15 & 15 & $\begin{array}{c}\text { \$FUYDUAG ) L } \\
\text { VOXG H } \\
\end{array}$ & 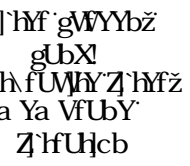 & \&KR्RQH & प्र & प्णा & प्णा & $\begin{array}{l}\text { +DFKIFKमH } \\
\text { DQm }\end{array}$ \\
\hline \% & $\square$ & 15 & 15 & $\begin{array}{l}\text { \$FUYDUAG } \\
\text { VOXG H }\end{array}$ & $\begin{array}{l}\text { 6DQG } \\
\text { ILQUDRQ }\end{array}$ & \&KR्RQH & प्र & प्राप & पाली & 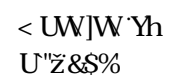 \\
\hline \%D & Q & 15 & 15 & $\begin{array}{l}\text { \$QDHRELF } \\
\text { DDQG } \\
\text { IDFXQUMYH } \\
\text { SRQG }\end{array}$ & $\begin{array}{l}\text { DUXLDMRQ] } \\
\text { WFNOQ } \\
\text { DLOMA }\end{array}$ & $1 \mathrm{RQH}$ & प्र & प्णि口 & प्राप & 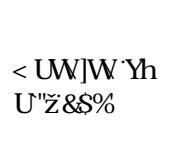 \\
\hline \&KLQD & $73 *$ & 15 & $\begin{array}{l}\text { 6FUHQL } \\
\text { DQG] UW } \\
\text { UP RYDO }\end{array}$ & $\begin{array}{l}\text { \$FUYDUAG } \\
\text { VOXG H }\end{array}$ & $\begin{array}{l}\text { 6DQG } \\
\text { ILOMDRQ }\end{array}$ & $1 \mathrm{RQH}$ & 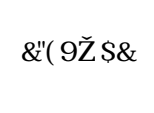 & प्राप & प्णा & 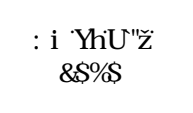 \\
\hline \&KIQD & : 73며 & 15 & $\begin{array}{l}\text { 6FUHQL } 1 \\
\text { DQG] UW } \\
\text { UP RYDO }\end{array}$ & $\begin{array}{l}\text { \$QDHREIF] } \\
\text { DQR[ IFER IF } \\
\text { SURFHW }\end{array}$ & $\begin{array}{l}0 \text { HP ELDH } \\
\text { XQUD LOUDMRQ }\end{array}$ & $\begin{array}{l}\text { 2] RQH] } \\
\text { FKRUQH }\end{array}$ & 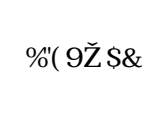 & an & पाण्रा & 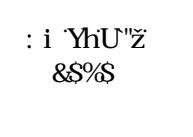 \\
\hline Ireland & $\begin{array}{l}\text { Plant } \\
\$\end{array}$ & 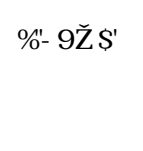 & $\begin{array}{l}\text { 6FUHQQ I } \\
\text { DQG UW } \\
\text { UHSDDURQ }\end{array}$ & $\begin{array}{c}\text { 6ХG H] } \\
\text { DFWYDMRQLO } \\
\text { R[ LDWRQ] } \\
\text { GLFK }\end{array}$ & $1 \mathrm{RQH}$ & $1 \mathrm{RQH}$ & 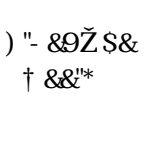 & पs & प्णा & $\begin{array}{l}\text { \&KHQ [MDQ] } \\
2009\end{array}$ \\
\hline Ireland & $\begin{array}{c}\text { Plant } \\
\%\end{array}$ & 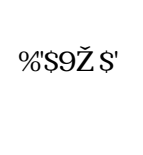 & $1 \mathrm{RQH}$ & $\begin{array}{c}6 \times \mathrm{QG} \mathrm{H} \\
\text { DFWYDWRQIQ } \\
\text { extended[ } \\
\text { aeration tanks }\end{array}$ & $1 \mathrm{RQH}$ & $1 \mathrm{RQH}$ & 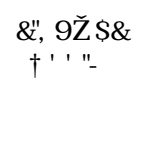 & $8 \pm 3.6$ & 1.54 & $\begin{array}{l}\text { \&KHQ [HDD II } \\
2009\end{array}$ \\
\hline Ireland & $\begin{array}{l}\text { Plant } \\
\&\end{array}$ & 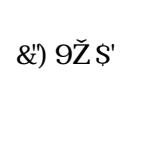 & $\begin{array}{l}\text { 6FUHQQ 四 } \\
\text { and grit } \\
\text { separation }\end{array}$ & $\begin{array}{c}6 \times \mathrm{QG} \mathrm{H} \\
\text { DFWYDWRQIQ } \\
\text { extended! } \\
\text { aeration tanks }\end{array}$ & $1 \mathrm{RQH}$ & $1 \mathrm{RQH}$ & 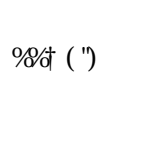 & पs प⿴囗十口 & पाण्ण & $\begin{array}{l}\text { \&KHQ [HMDQ] } \\
2009\end{array}$ \\
\hline Ireland & Plant & 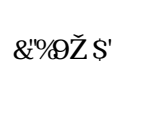 & $\begin{array}{l}\text { 6FUHQL } \\
\text { DQG UW } \\
\text { VHSDDMRQ S }\end{array}$ & $\begin{array}{l}\text { \%RLLP [FRDUAG] } \\
\text { SHFROUD ILOHA }\end{array}$ & $1 \mathrm{RQH}$ & $1 \mathrm{RQH}$ & ¿s ए & पs & $\square$ & $\begin{array}{l}\text { \&KHO पMDQ⿴囗⿰丨丨 } \\
2009\end{array}$ \\
\hline , VDHO & $\begin{array}{l}\text { 300W } \\
\$\end{array}$ & 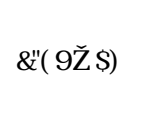 & $\begin{array}{l}\text { 3UP DU1 } \\
\text { VHMQg }\end{array}$ & $\begin{array}{l}\text { \$FUYDUAG } \\
\text { VOYG H }\end{array}$ & $\begin{array}{l}\text { 6RZ[YMQG] } \\
\text { ILQMDMRQ }\end{array}$ & \&KRUQDURQ & 밈 & प्रा & 0.24 & 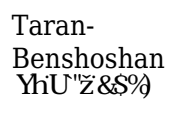 \\
\hline 6SDQ & $\begin{array}{l}\text { 300W } \\
0\end{array}$ & 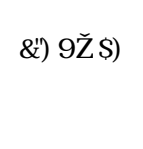 & $\begin{array}{c}\text { 6Fu+HQg m } \\
\text { and grit } \\
\text { separation, } \\
\text { sedementation }\end{array}$ & $\begin{array}{c}\text { \$QDHREIF } \\
\text { GI HMRQM } \\
\text { VHGP HQMDURQ }\end{array}$ & $1 \mathrm{RQH}$ & 89 & NR & 0.4 to 0.8 & 2.27 & 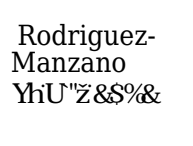 \\
\hline 6SDQ & 300W & 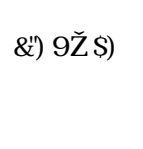 & $\begin{array}{l}\text { 6FUHQQ 四 } \\
\text { and grit } \\
\text { separation, } \\
\text { sedementation }\end{array}$ & $\begin{array}{c}\text { \$QDHREIF } \\
\text { GJ HMRQM } \\
\text { VHGP HQMQMRQ }\end{array}$ & $\begin{array}{c}\text { 6DQG } \\
\text { ILQUDMQQ }\end{array}$ & 89 & NR & 0.4 to 3.6 & 1.75 & 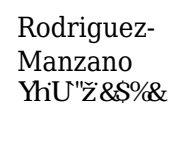 \\
\hline $86 \$$ & $\begin{array}{l}\text { 30QW } \\
\$\end{array}$ & 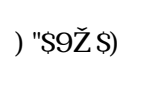 & 15 & $\begin{array}{l}\text { \$FUYDUAG } \\
\text { VOXG H }\end{array}$ & $1 \mathrm{RQH}$ & \&KRUQDMRQ & $7.4 \mathrm{E}+01$ & $1.3 \mathrm{E}+01$ & 0.71 & 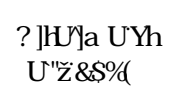 \\
\hline $86 \$$ & $\begin{array}{l}\text { 3DQW } \\
\%\end{array}$ & 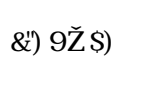 & 15 & $\begin{array}{l}\text { \$FUYDDAG } \\
\text { VOKG H }\end{array}$ & $1 \mathrm{RQH}$ & \&KRQUQDMRQ & $1 \mathrm{E}+02$ & $1.2 \mathrm{E}+01$ & 0.81 & 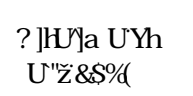 \\
\hline
\end{tabular}




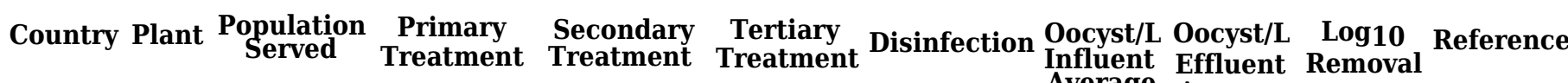
Average Average

\begin{tabular}{|c|c|c|c|c|c|c|c|c|c|c|}
\hline $86 \$$ & $\begin{array}{l}\text { 30QW } \\
\$\end{array}$ & 15 & $\begin{array}{c}\text { 3UP DU: } \\
\text { v-dimentation }\end{array}$ & $\begin{array}{l}\text { \$FUYDUAG } \\
\text { sludge, } \\
\text { secondary } \\
\text { VHGP HQMAMRQ }\end{array}$ & 15 & $\begin{array}{l}\text { Chlorination/ } \\
\text { dechlorination }\end{array}$ & 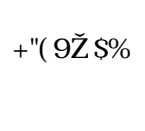 & 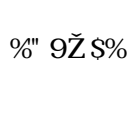 & $\begin{array}{c}0.76 \\
\pm 0.22\end{array}$ & $\begin{array}{l}\text { Schmitz } \\
\text { et al., } 2018\end{array}$ \\
\hline $86 \$$ & 3\%OW & 15 & $\begin{array}{l}\text { 3UP DUP } \\
\text { v-dimentation }\end{array}$ & $\begin{array}{l}\text { Trickling } \\
\text { filters, } \\
\text { secondary } \\
\text { VHGP HQMDMRQ }\end{array}$ & 15 & $\begin{array}{l}\text { Chlorination/ } \\
\text { dechlorination }\end{array}$ & 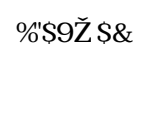 & 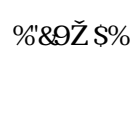 & $\begin{array}{c}0.96 \\
\pm 0.38\end{array}$ & $\begin{array}{l}\text { Schmitz } \\
\text { et al., } 2018\end{array}$ \\
\hline $86 \$$ & $\begin{array}{l}\text { 30QW } \\
\text { C }\end{array}$ & 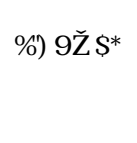 & $\begin{array}{l}\text { Dissolved } \\
\text { Air } \\
\text { Flotation }\end{array}$ & $\begin{array}{c}\text { } \text { DCWy H } \\
\text { Bardenpho, } \\
\text { secondary } \\
\text { VHGP HQMDURQ }\end{array}$ & $\begin{array}{c}\text { Disc } \\
\text { Filtration }\end{array}$ & $\begin{array}{l}\text { Chlorination/ } \\
\text { dechlorination }\end{array}$ & 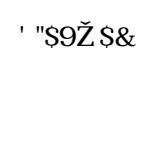 & 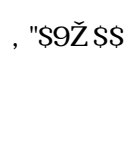 & $\begin{array}{l}\mathrm{y} \\
\pm 0.39\end{array}$ & 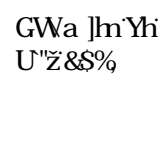 \\
\hline $86 \$$ & 300W & 15 & $\begin{array}{c}\text { 3UP DU: } \\
\text { Vydimentation }\end{array}$ & $\begin{array}{c}\text { 3VHXGR } \\
\text {-bardenpho/5- } \\
\text { stage } \\
\text { Bardenpho } \\
\text { secondary } \\
\text { VHGP HQMDQRQ }\end{array}$ & NR & $\begin{array}{l}\text { Chlorination/ } \\
\text { dechlorination }\end{array}$ & 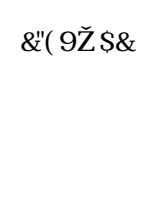 & 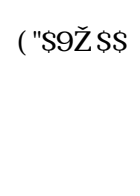 & $\begin{array}{l}\mathrm{a} \mid \mathrm{m} 2 \\
\pm 0.54\end{array}$ & 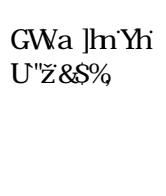 \\
\hline $86 \$$ & $\begin{array}{c}\text { 30QW } \\
\square\end{array}$ & 15 & 15 & $\begin{array}{l}\text { Activated } \\
\text { sludge, lime } \\
\text { treatment }\end{array}$ & $\begin{array}{l}\text { 6DQG filtration } \\
\text { Upflow } \\
\text { FDERQ } \\
\text { adsorption }\end{array}$ & In \&KQUUQDMRQ & 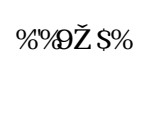 & 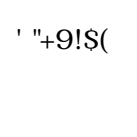 & 4.47 & $\begin{array}{l}\text { Rose et } \\
\text { al., } 2001\end{array}$ \\
\hline
\end{tabular}

$15 \square \mathbb{1}$ RVDGHSRUAG

\subsection{Excreta and Wastewater Treatment}

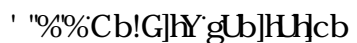

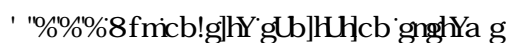

\&RP SRWLI EDMHGLMDQLWWRQLM WAP VDDQR LNQRZ QDDV

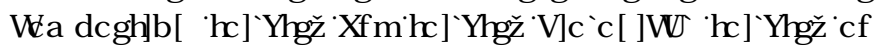

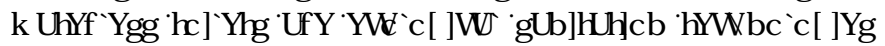

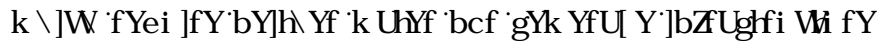

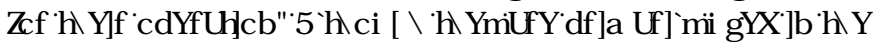

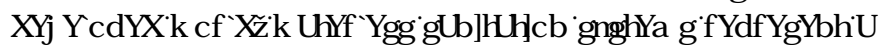
YLEOFIRQURQIRUDUHDVZ KHUHSRRUMDQLUWRQTH LWMDQG

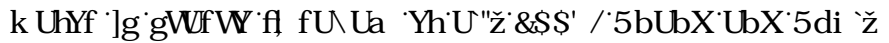

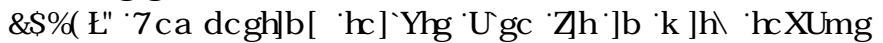

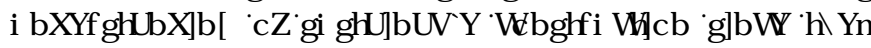

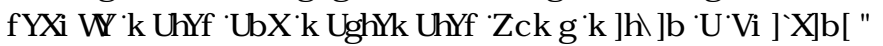

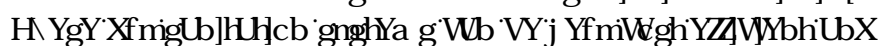

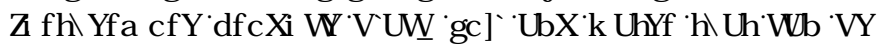

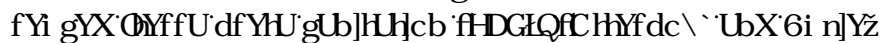

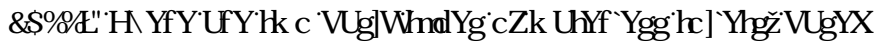
RQZZ KHMKUTMY [WHDVELRVRQGZ DWHEI ELRGH UDGDRQRU GHKI GUDMRQ]C/dRGH UDGL] [MRLOWN [SURP RVA [SDMKRJ HQ

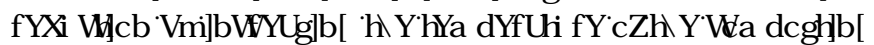
SLOYYRDVIKL KDVM]

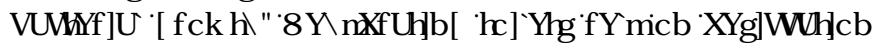

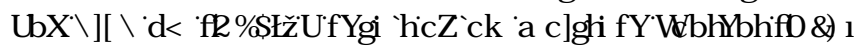

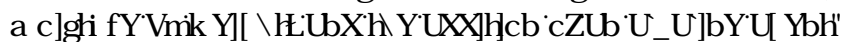

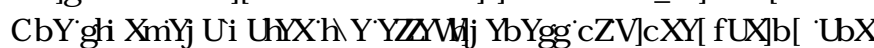
GK। GOMG [ZDMAOMYYRLOMLQUHGXFL] [Cryptosporidium

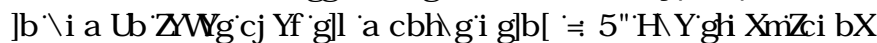

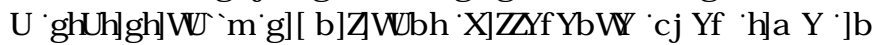
Cryptosporidium GHMAFVAG[Q[पKK [GHK। GUDQ] [M WAP

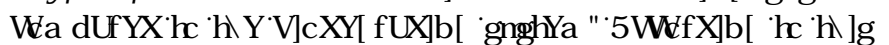

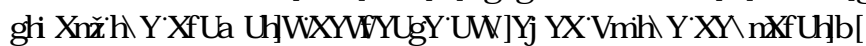

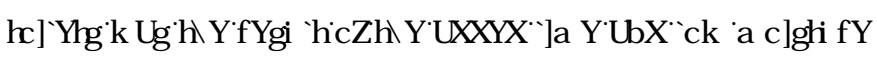
OYHQM

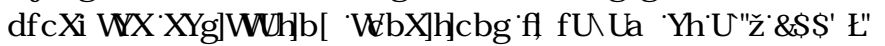
\%RVLHQYLRQP HQVDOIDFVRUVTKL KES+ DQG GHMFFDMRQDUH

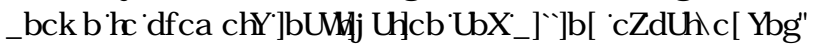

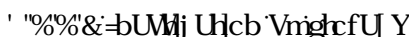

\&RP SRWQ IMLQFUHDMQ Q FRQMGHUGDT RRGZD IRU

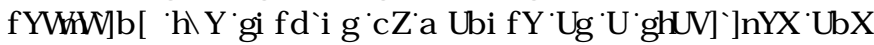

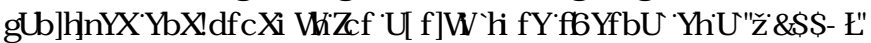

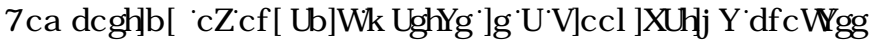

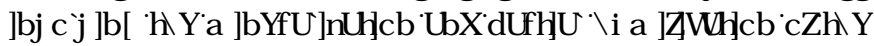

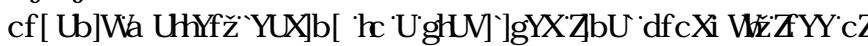
SKI VRVR[ LFLW DQG [SDMKRJ HQVDDQG Z LVK [FHUVDQQKXP IF

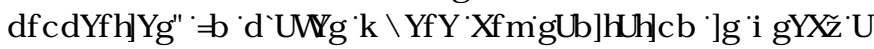

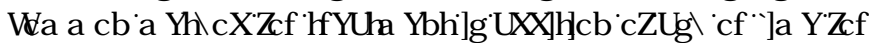

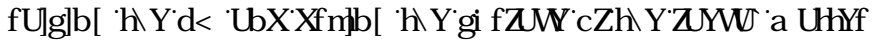

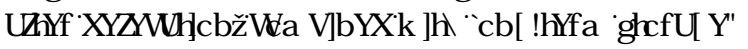

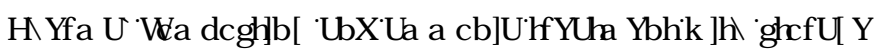

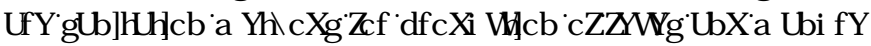

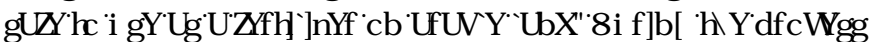

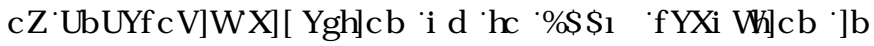
Cryptosporidium YLELOWLRFFXUZ LKLQDDMRUWSHURGR

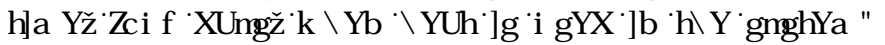

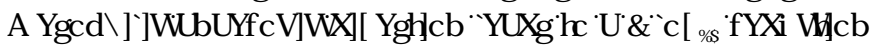
LQRRFI WUXXYLYDQZ KLOHLYKHUP RSKLOF DQDHRELF GGL HMRQ

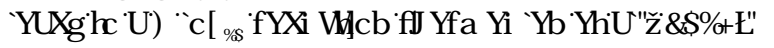

7KHLXUYLYDQR EC. parvum[QDDQP DQP DQXUHMDQGP DQXUH VQWHMKDVEHQR WAQJWXGHGXQGHFRQNRCHGODERDURU FRQGLVRQVDT KHMH [WXGHN [KDYH [GHP RQWWDWHG [VKDW UP SHOXXHMEHZHQTIDDQG

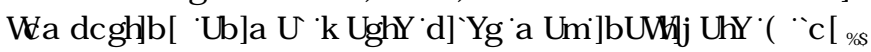


पI

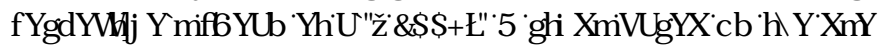

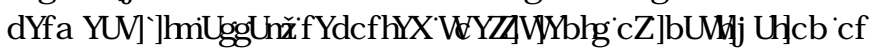

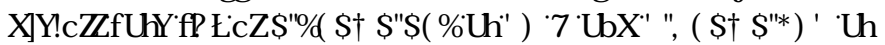

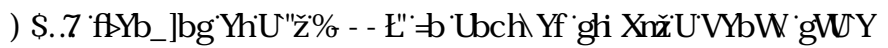

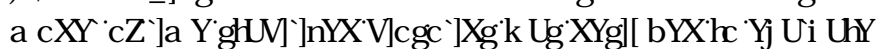
UXHSHUMUAOFH IRI LYLDQIEDFUAUDQDQGSDUDMUF[SDURR] HQV]

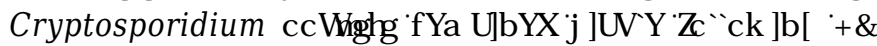

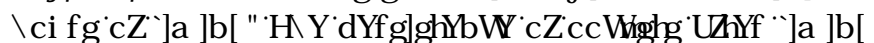
VXJ J HMAGLMKDVC. parvUT ZZRXQEEHDEEHMAUFKRLFHDMKDQ Salmonella DMLQGFDMRLIRULYYDODMG [ELRVROGLOMAQGG

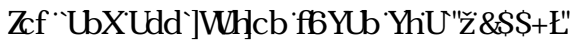

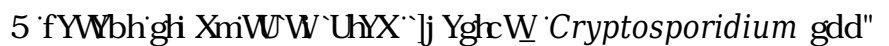

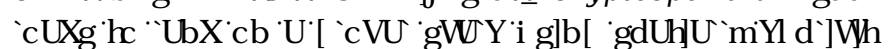

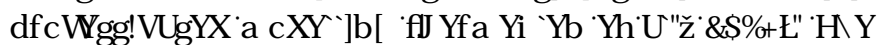
WXG [HYYHDOGDCIRUDDI REDOC ryptosporidium RRF। WWRDG IURP QOYHMRFNP DQXUH RI

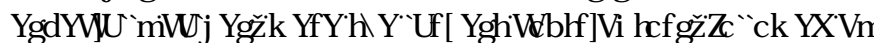
FKIFNHQVDQGSW VII6SDWDOGUIHHQFHMZ HU [QQNHGURLDQP DO

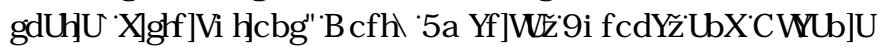

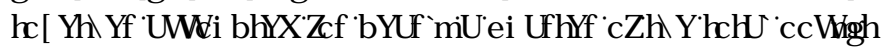

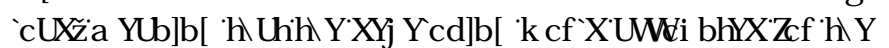

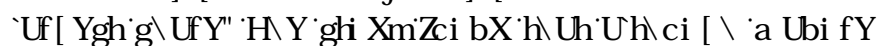
WRLI HIKDOYHGRRF WTRDGVIP DQXUHUM-DUT HQMIHNSHFDQ

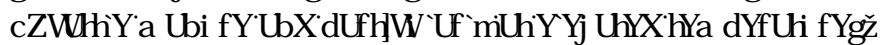

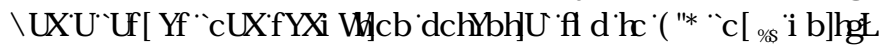

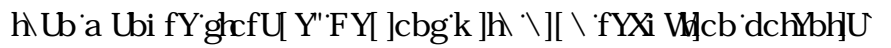

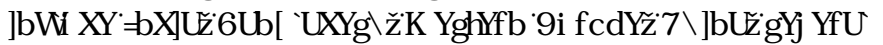
FRXQMHMLQW\$IUFD

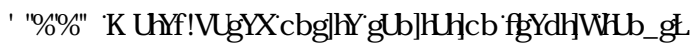

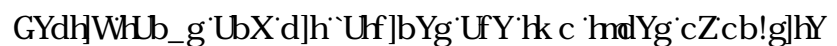

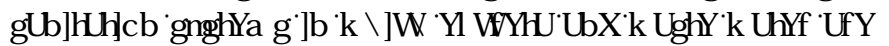

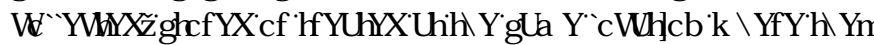

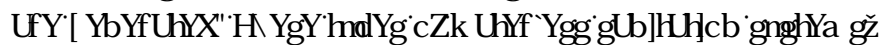

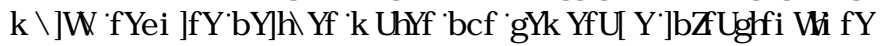

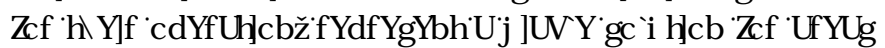

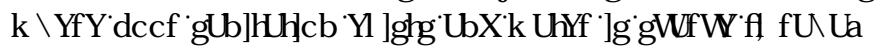

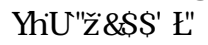

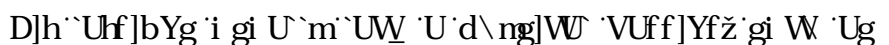
FRQFUHMADIEHVI HHQLWRUHG[H[ FUHP HQWIDQGLRLQDQGRU

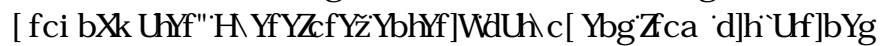

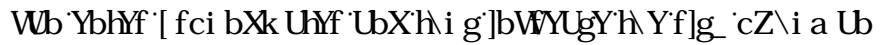

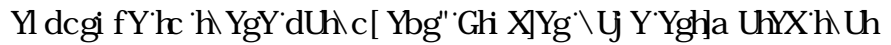

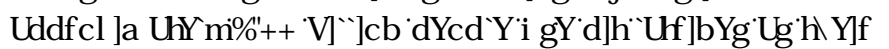

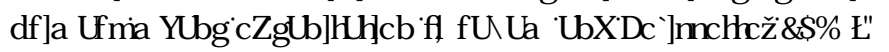

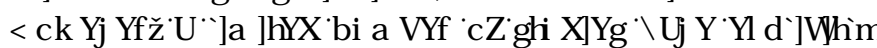
H[ DP LQHG [QQNW EFHZ HHQ] URXQGZ DVHU [SRQXVRQ [DQG

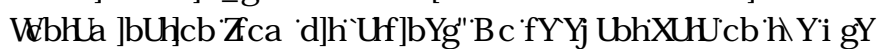

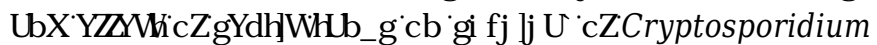
RRFI WWRURFFXUHQFH[ZHUHDRXQGTHRZ HYHUSLLDOMQHN

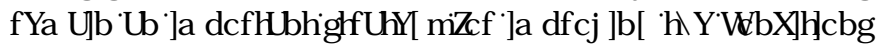

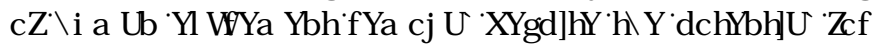

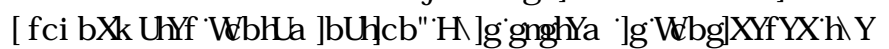

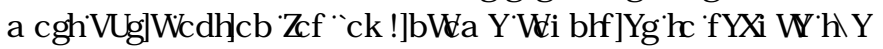
OYYHQRI RSHQGGH HFDMRQDQG] S SDQGDFFHMIYRDP SURYHG

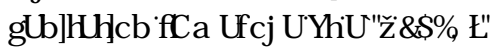

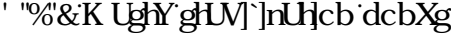

DMA[WDELQ] DMRQ [SRQGVII: 63VDLHP SQRI [QDUXUO SURFHMHVDYR [WHDWGGP HMF [Z DWAZ DMAUTMHSVDMADDQG VOKG HDDVIZ HODVDDQP DQRULQGXWUDQZ DWAVDOG DUH IUHTXHQVQ [XVHG DQFRP ELQDVRQZZ LIK RVKHULVDLVDVRQ

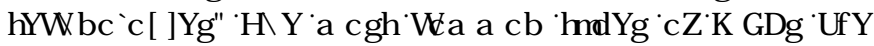
DQDHURELF [SRQGYD DFXQDUYHISRQGYIP DUXUDRQ [SRQGV] DHUDVAG [SRQGVDDQG [KL KJDVHDQ DOSRQGV 3DMKRJ HQJHP RYDQIURP [ZDMAZ DMAUR, 63VDQG[ZDMA

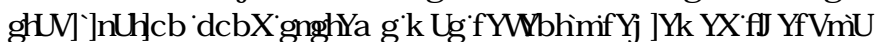

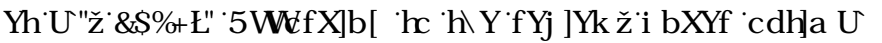

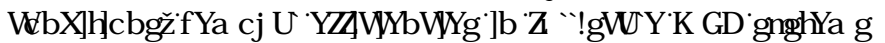

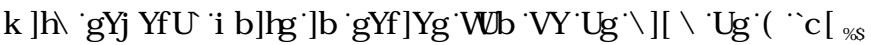

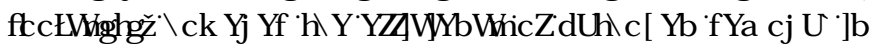
IXQMFDOIK WAP VIVIKL KQ TYDUDEOUDQGLQSLDFWFHP DQ

63[M WHP VDFKLHYHRQQ

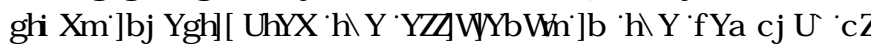
Cryptosporidium RRF WMLDDG 63[M WAP [RUP HGEI [YZ R DQDHRELF [SRQGVIDIDFXQDWY'SRQGDQGDP DUXVMRQSRQG

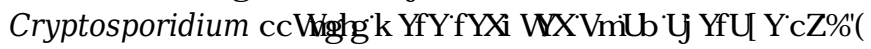

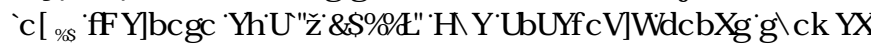

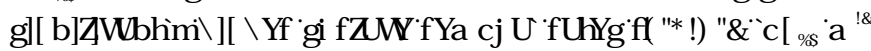

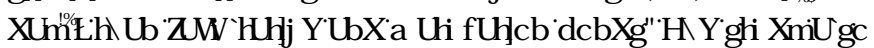
GHP RQWWDAG[YKDWVXQQ] KWIDQG [ZDMAU[SKI UFRFKHP IFDO FRQGLWRQVZZHUHLMKHIP DQRDFVIRURQ QHQFLQ [C. parvum

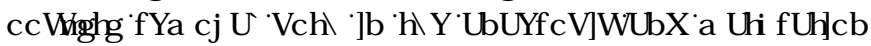
SRQGVIZ KHUHDVLRVKHUTDFVRUVIDNH[SUHGDURQRULQDUUDO

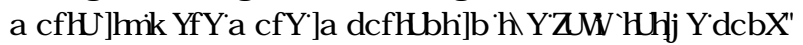

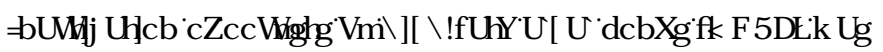
Kप KHU पKKDQ ZZ LLK GFRQYHQWRQDQZZ DWHZ DMAUTWHDVP HQW

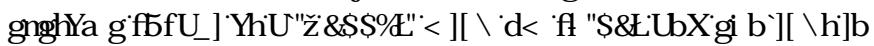

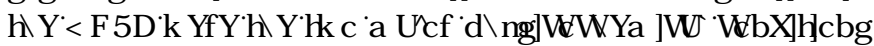

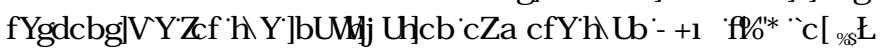

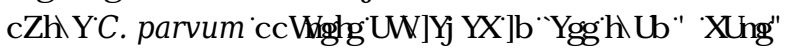

0 IFLRELQUHP RYDQUDAMZ HUTWXGHGLQP DUXUDRQSRQGV

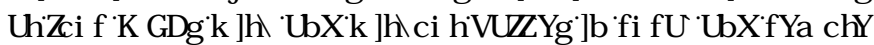
FRP P XQLWHLQ\$ XWLOD『Cryptosporidium VSS $\square R R F$ WLL

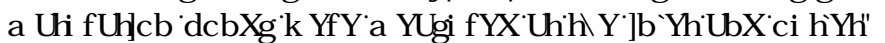
/ RZ QQXP EHUVR Cryptosporidium RRFI WMZHUHGHAFWGD

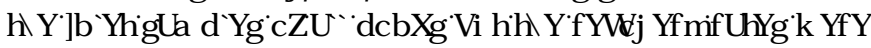

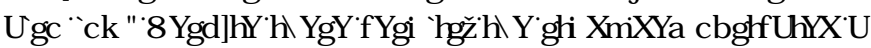

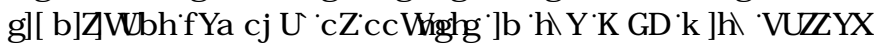

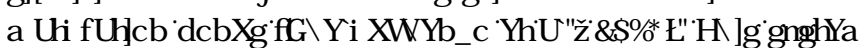
FRP SUMHGDSSUP DU TDFXQDUYHSRQG ШEDIOHGP DUXUDRQ SRQG[ZLVK

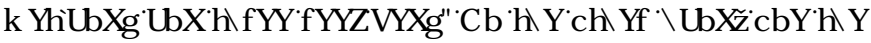
QRQEDIOAKK WAP VZDVGHSHYMKDQH SHFWGZ LLAP DUNG

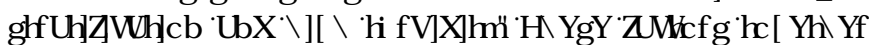

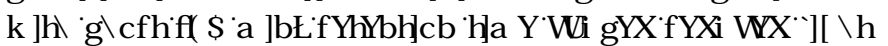

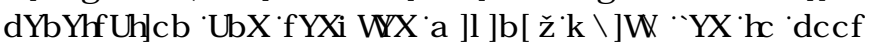
UHGXFWRQRI RRFI WWII R JHGXFWRQLQ [MKH QXXP EHURI Cryptosporidium ZDVREVHUHGLQDORVKHUQRQEDIIOIG

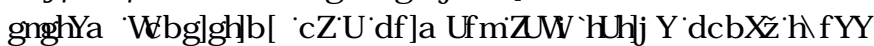
FRQMFXUYHP DUXUMRQSRQGMRIMP LDUM] HШDQTMMP DMAG

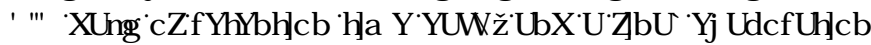

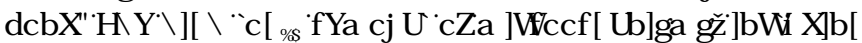
SLRVR] RDQRRR WWIREVHUYG DVMKHEEDI OHGLM WAP [ZDV

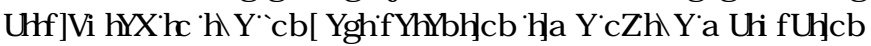

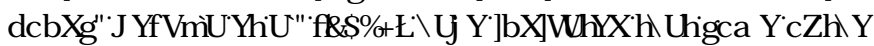




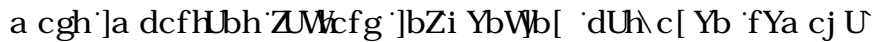
HIIFLHQF [RQ] 63VROFOGH[KI GUXQF [UHMOMRQ[MP H[DQG

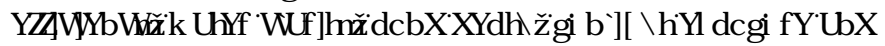

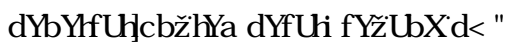

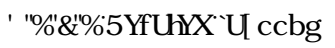

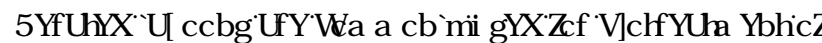

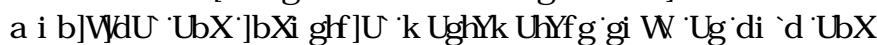
SDSHUP LQ] I XHQW] 6 XFK [D] RRQVIKDUERU[FRP SOH[

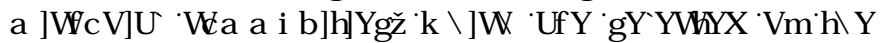
SKI UFRFKHP IFDOSLRSHUNMRRI [MKH[ZDMAZ DMAUTMKH[GMJ Q DQG [RSHUDWRQ [RI [MKH[DJ RRQQDQG [MKHDP EUHQW

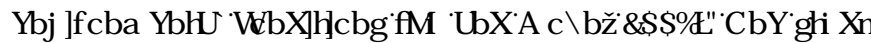

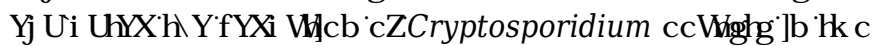

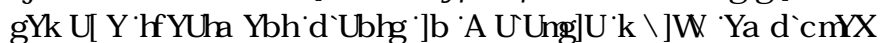

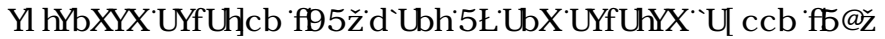

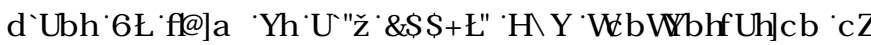

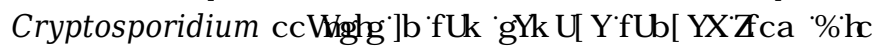

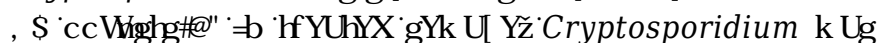
IRXQGRQR

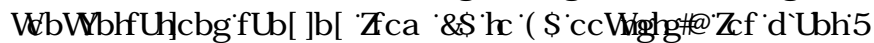
DQG

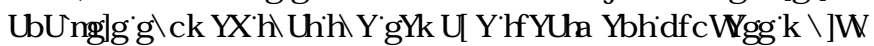
HP SQR HG [H[ VHQGHG DHUDMRQ [M WAP [SURYLGHG P RUH

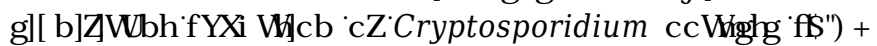

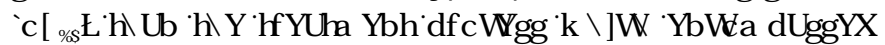
DHDUAG(ه) RRQWس

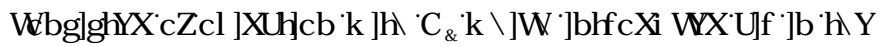
IRLP [RI TLQHEXXEEOMLMXLX] K[YXEP HY HG[GUIXVHUD] LQH

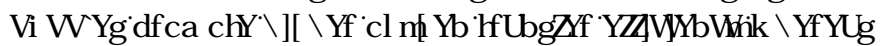

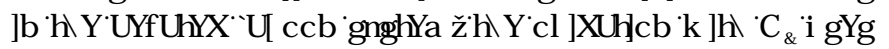
VXUDFHDHDMRUYIRSLRYLFIDU]

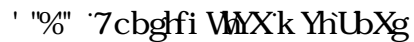

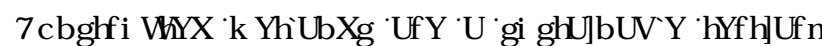

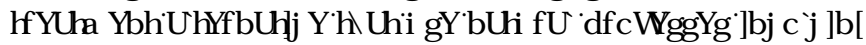
Z HMOG[YH HWWRQTIRLQIDQG[MKHUDDWRFLMAGP IFURELD DVVHP EDJ HV INR ILP SURYH [Z DWHU पTXDQWY

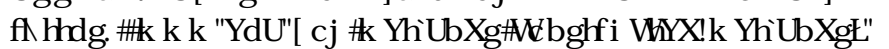
HMOQGEFHKDYIRUDDG[HILFLOFI FRQFHUQQ [ZDMAZ DUAU WHDVP HQVIVVP DLQQ [OQNHG[VRP DFURSKI WA[FRP SRYURQ]

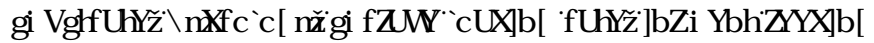
P RGHDP LFURRY DQLVP TDYDLOELLWTDDQG CVHP SHUDXUH

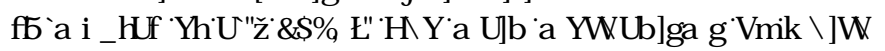
P IFURRY DQLVP VIDQG RUKHU[SRCXVDQW DUH]HP RYHGLQ FRQWXXFUAGZZ HODQGVIKDYHEFHQGHMUEHGRQ[SUHYRXV

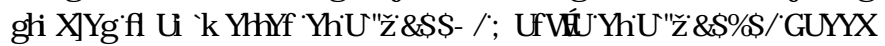
DQG 6 XQ1]

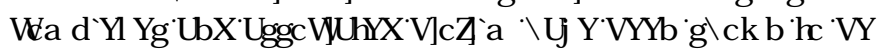

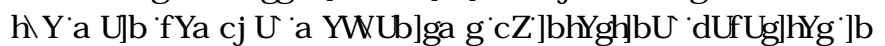

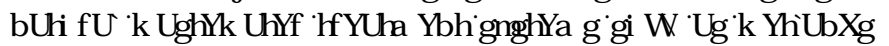

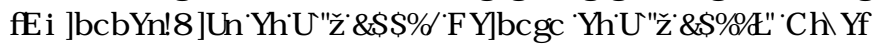
P HFKDQLVP VIRI [UHP RYDQFRXG EFH[MHGLP HQVDURQDQG

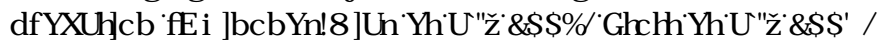

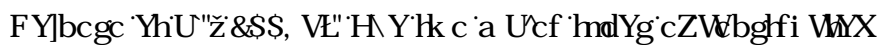

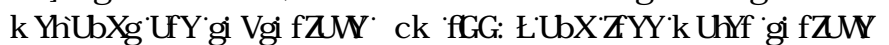

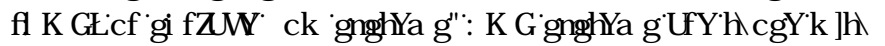

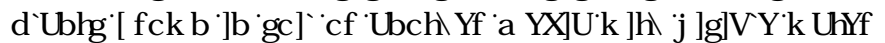

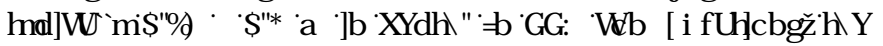

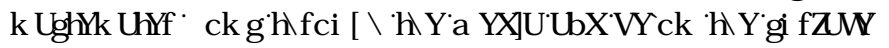

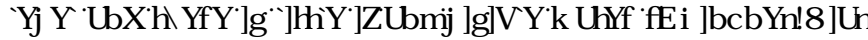

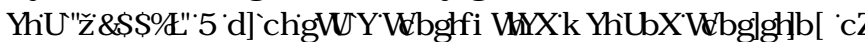

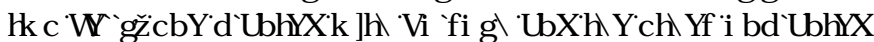
EDUH UHP RYL] [Cryptosporidium RRF। WW TURP [DZ [M-ZDJ H

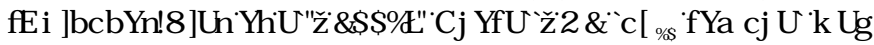
DFFRP SQVKHGEI [ERVKLVKHIVXUDFHIRZZTURP [SDQMAGFHO

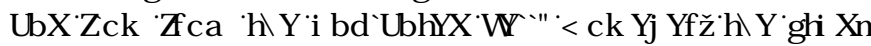

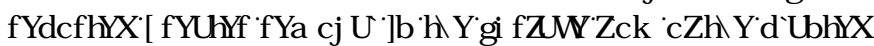

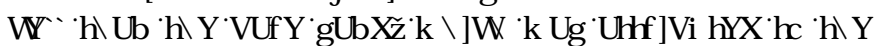
HQKDQFHP HQWRI [MHGLP HQVDWRQRRUDDGVUSWRQLEI [MKH

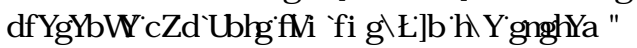

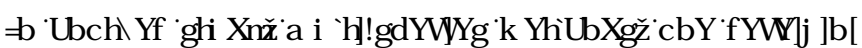

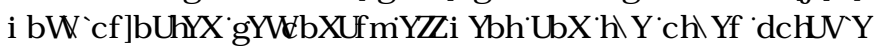

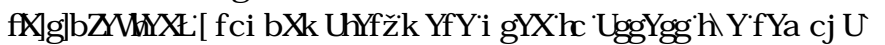
R [Cryptosporidium RRF WW W KXUMRQ [MWDQm XQFKQRUQDUAGLMFRQGDU [MHDMAGLZ DMAZ DMAUIKDGEFHHQ SUHYIRXVQ[MU-DUAGE] [SDWD] HUMLLXJ KDDGXFNR HGGFRYHUG

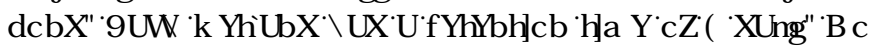

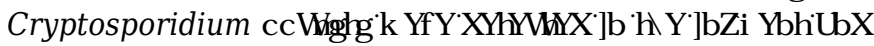

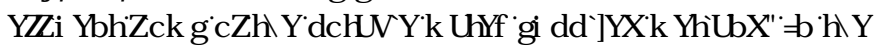

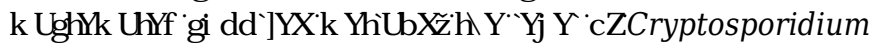

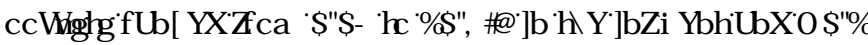
VRप

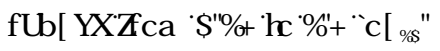

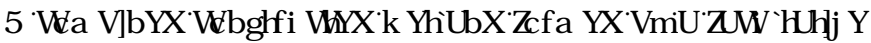

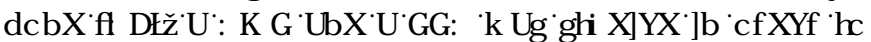

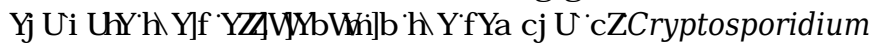
DQG[QGLFDMRUP IFURRY DQLVP VIDQG]MR GGHMALP LQH[MKHU

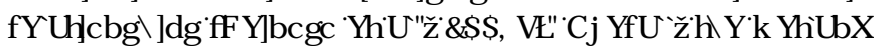

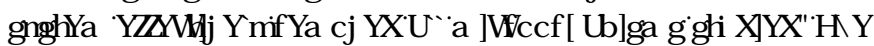

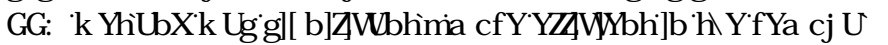
RI [Cryptosporidium RRF। WW

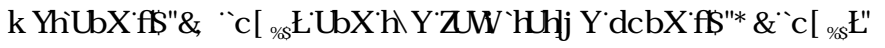
1 R[Cryptosporidium RRFI WWZZHUH[GHMFVHG[Q]MKH] LDO

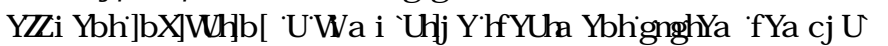

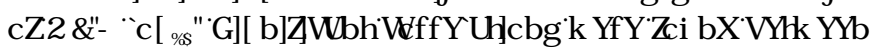

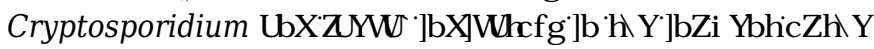

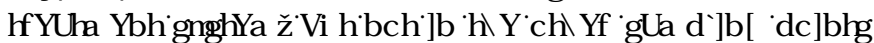

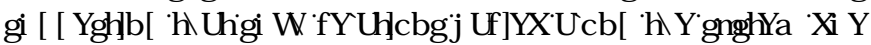

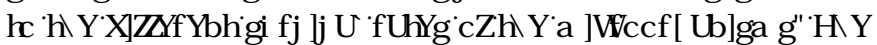
WXG [GHP RQWWDMAGLKKDWVMKHERP ELQHG[XVHRI GUIHUHOW

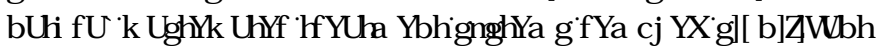
DP RXQW[RI [SDUKR] HQIF [DQGLQGLFDRUIP LFLRRY DQLVP V] +RZHYHUTMKHMHUHGXFURQVZ HUH[QRVMHQRX] KDRUDLMAHW UHXYHRIVMKTLLDQHIXKOWT

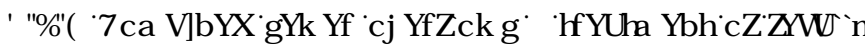
SRCOMAGWMRLP ZDUA

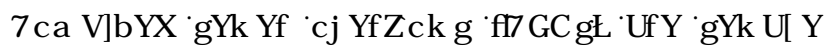

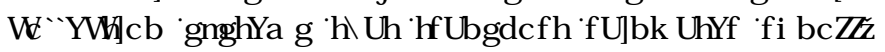
GRP HMF[M-ZD HWDQGLQGXWLDQZ DMAZ DMALQMRRRQHSLSH]

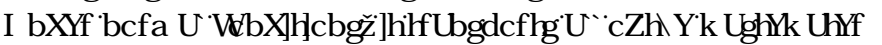

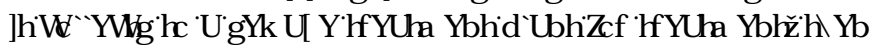

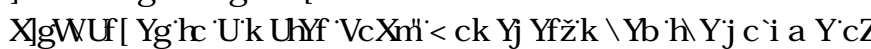

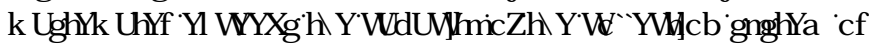

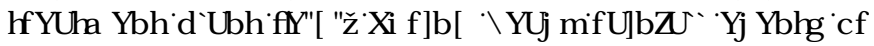
VQRZ P HOWTIKLVIXQRII GVGGLVFKDY HG[GLHFVQ [MR [QHDEI WU-DP VTUYHUVIDQGRRYHUZ DUALERGHMFDXVQ [DGYHUH 


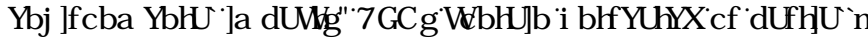
WH-DUAGIKXP DQIDQGIQGXWUDQZZDMAIIVR[ IFIP DUAUDQVIDQG GHEUVDVZ HODVMMRLP Z DVHUT KH[8 6( 3\$ [KDVIGHQW LHG

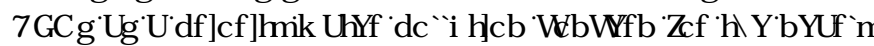

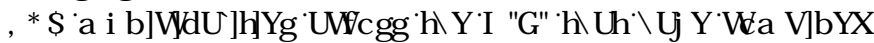
VHZ DJ H $\square \mathrm{M}$ VWHP $\mathrm{V}$

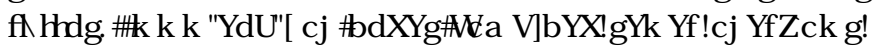
FVRVI

\$[WXG ROYHWY DUAGWMHFRFFXUHOFHRICCryptosporidium LQ

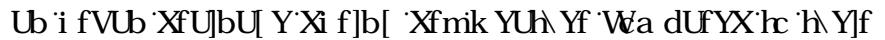

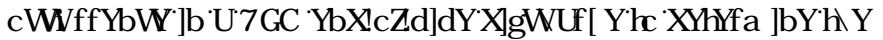
RDGLQ] [SRUAQWDQDQG[YKH'SRUAQWDOKXP DQIKHDOKIP SDFW

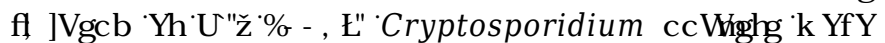
FRP P RQQ REVHUYHG]Q [MKH[XUEDQLMUHDP [GXUQ] [GU

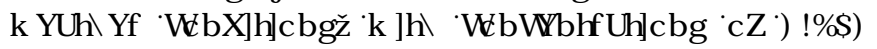

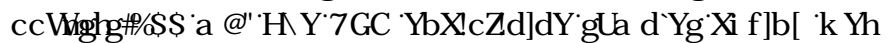
Z HDVKHU एFRQGLWRQV [GLVFKDU HG [KL K [OHYHQ [RI

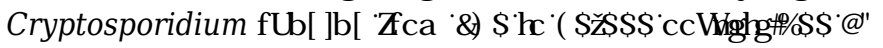
7KHLWXG [GHP RQWWDVHG[IKDWL62 VIFDQ[M] QU LFDQVQ FRQWUEXVA[MRTMKH[RDGRI \&UU SVRVSRUGLXP TQDDP ELHQW

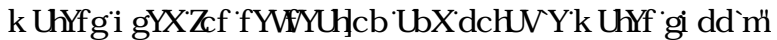

\$QRVKHULWXG [RI [MKH[\&KLFD] R[\$UHDG DMALZDI [6। WAP

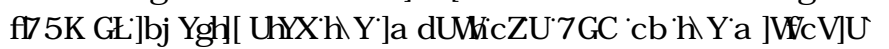

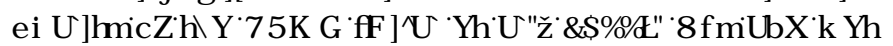

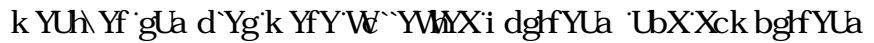

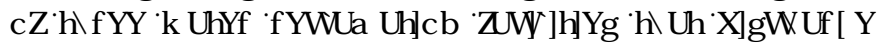

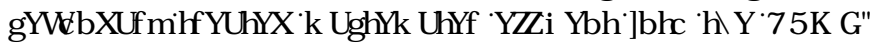
Cryptosporidium HQXP HDMRQLQF QGHGRQ HFWRXVRRF WW

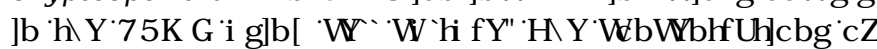

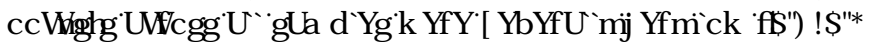

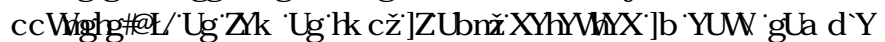
DQDQ ] HGD RUGGU [Z HDMKHU\&\$: 6[MDP SOMIQRLQI HFURXV RRF WWZ HUHGMMFUA IR YHDODIMKHFRP ELQHGZZ HMDQGGU Z HDMKHUSHFHQMV HRIIQ HFWRXVIRFLZZDMHMP DUAGIREEH

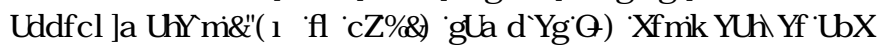

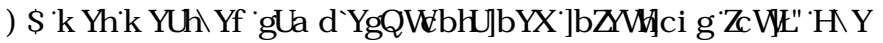

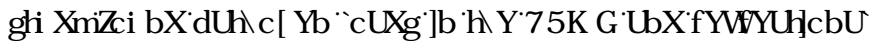
XVHUTMVIRUF*, ,

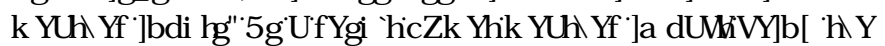

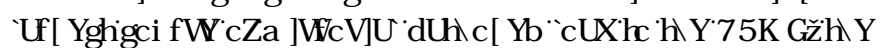

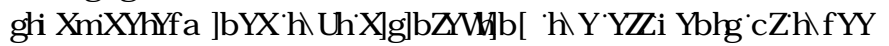

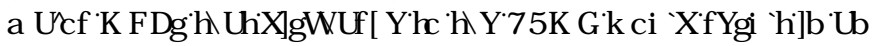
H[ WHP HQ LYP DQU]HGXFWRQLQ[MKHLD] J UH DMA]UHFUHDMRQ

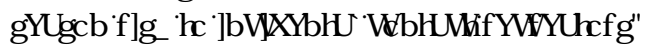

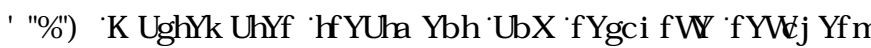
IDFLOMHN

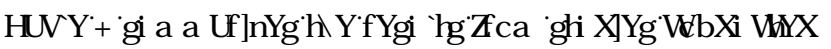

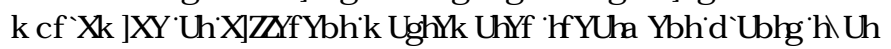
SURYLGH [GUIHUHQW TOHYHO IRI GWUHDWP HQWDRU

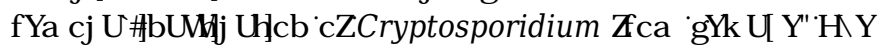
UHP RYDQH IIFLHQF IRI [Cryptosporidium RRFI WW[GXUQ VIZD HUMHDWF HQMLSLRFHMHMIVYYUDEOIDQGGHSHQGYXSRQ

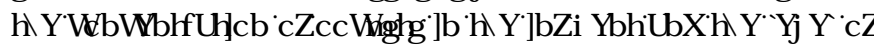

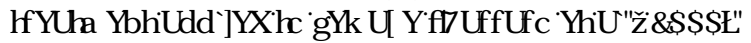

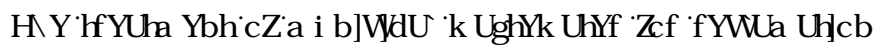

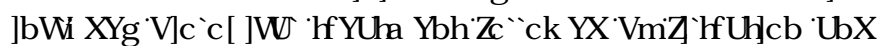

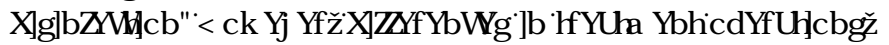

YDUDMRQVLQTLOMUGMI QMIIDQGGMQ HFURQDSSSLRFKHMFDQ SLRGXFH[HI OHOWRI [YDU LO TXDOWШ

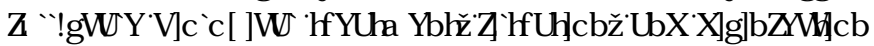
IRU]UHP RYDORI [Cryptosporidium RRFI WWDQR [RVKHU

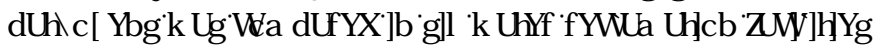

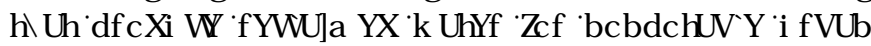

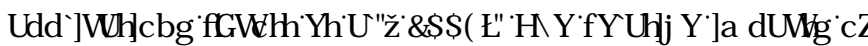
[RDGLO] [FRQGLVRQV][SLRFHW [GHM] Q]DPQG RSHUDWO] SDUDP HMAUVRQ[UKHUUP RYDQLQDFWYDURQRI RRFI WWZZ HUH

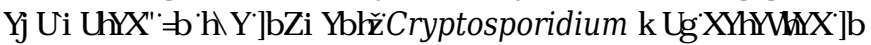

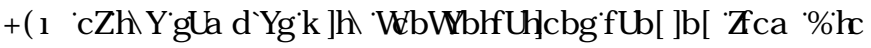

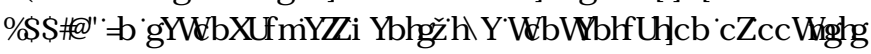

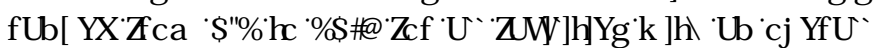

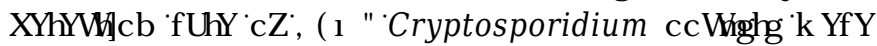
GMAFUAG[Q]

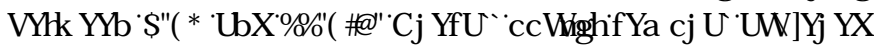

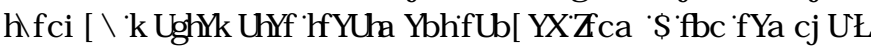

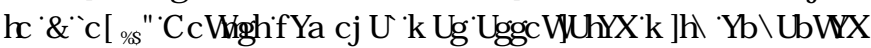

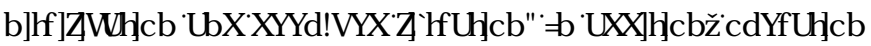

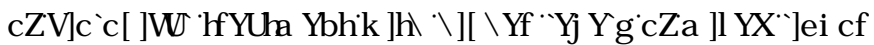
VXVSHQGHGLVRQGVDQG[RRO HUP HDQFFHQUHMGHOFH[MP HV

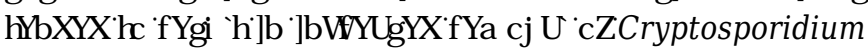

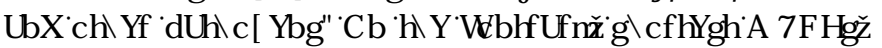

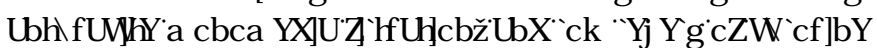

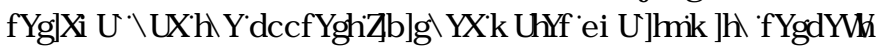

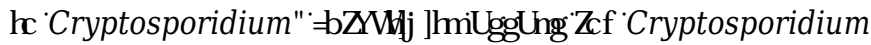

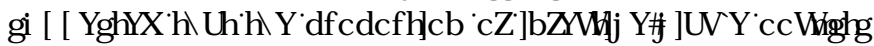

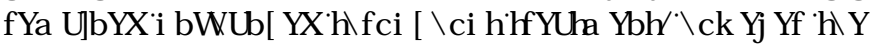

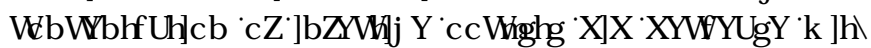
LQFUHDM] [GH] UHHRI [WHDWF HQWV7 KH[MXG [GHP RQWWDAG

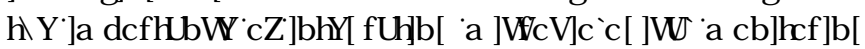

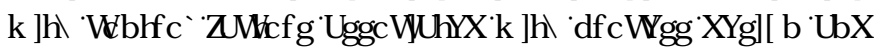
RSHDWRQVIDVDP RUH[REXWMSSURDFKDRLHQVXUQ] [MKH VIHMRIUFOIP HGZ DUAU]

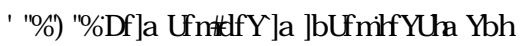

3UP DU [KHDVP HQWIVZQHIILHOW] RU]HP RYDORI Cryptosporidium IURP [M-ZD H[W] KLIP RLHDQG[ REHURQ]

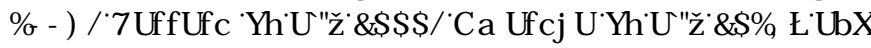

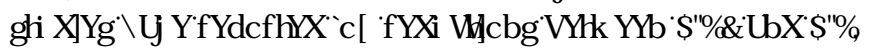

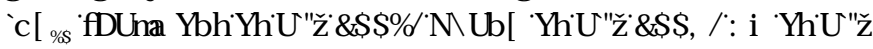

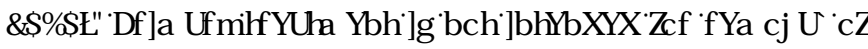

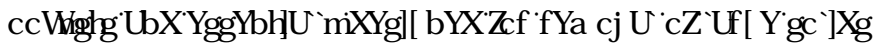

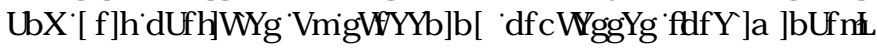

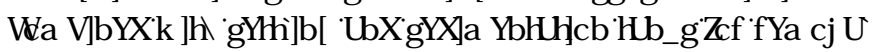
RILXVSHQGG]VRQGVDQGLMRP HRY DQFP DMAUT* HUEDDQG 3HSSHU⿴囗十ापा

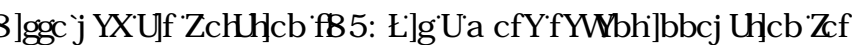
UHP RYLD [VXVSHQGHGLVRQGVIURP [M-ZD] HIZKLFK[VLORZ

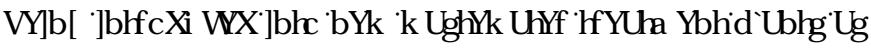
DQDDOHALDMYHCYR FRQYHQMRQDQSUP DU [MHGLP HQVDVRQ SLRFHMHMD \$) FFOULUVIFP RYHIXXVSHQGHGLRQGVP RUH USLGQLMKDQJGRHMFROYHQMRQDQSUP DU IMHGIP HQMUMRQDDQG

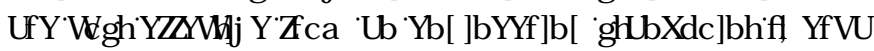

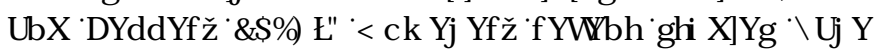

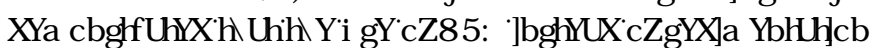

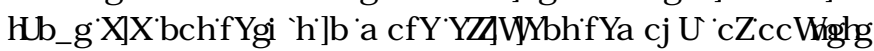
IURP [YHZD HШ6FKP LYGHMPQ 


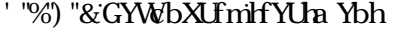

7KHLP DNRUW [RI [SURVR] RDQUHP RYDQRFFXUV[GXUO]

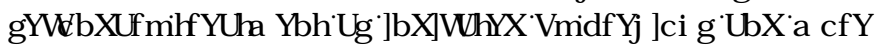

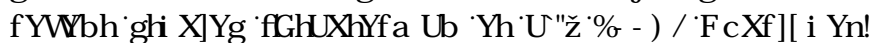
0 DQ] DQR [HMDQIس]

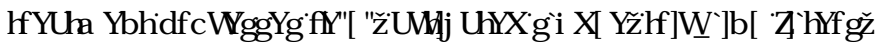
ZDMA[MIELL] DWRQ[SRQGMIIFRP P RQQ TQYROHMLELRQI IFDO

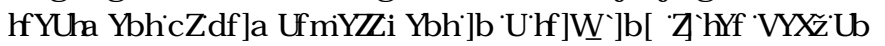

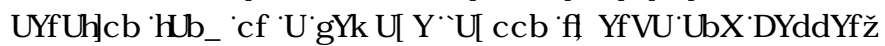

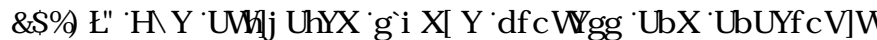

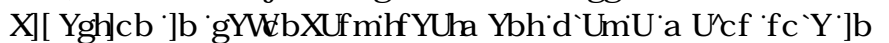
UP RYDQDQG LQDFWYDWRQRI RRFI WW

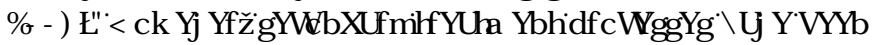

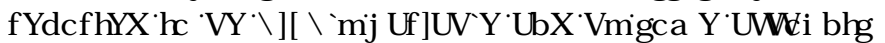
LQHIHFUYHПQ]HP RYL] [Cryptosporidium [\&KDXUHMHMDQ]

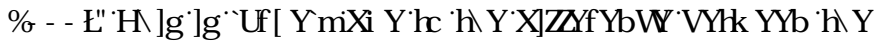
VHGP HQMGMRQYYHORFLWHR [SURVR] RDQRRFI WWZLLYUHSHFW

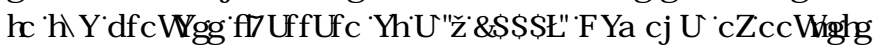

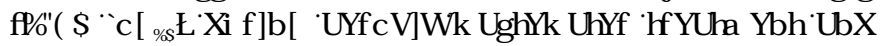

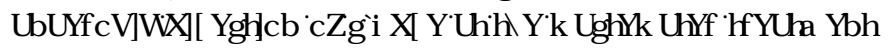

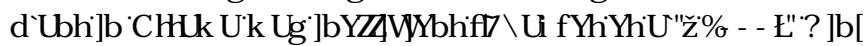

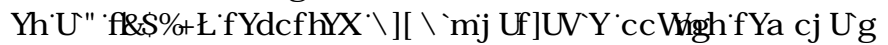

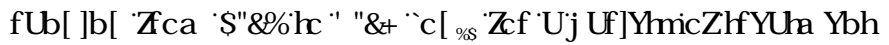

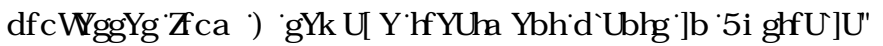
5 HP RYDODFURWDMHFQGDU IWHDVP HQWSLRFHWHV Z HUH

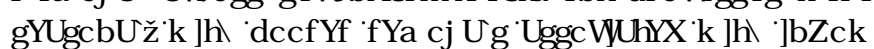
YDUDELQWDZ DJ RRQ [M WAP V IIZ DWHZ DMAULWDELQ] DMRQ

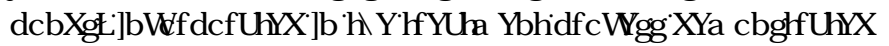

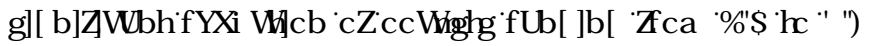

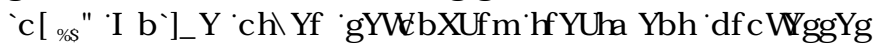

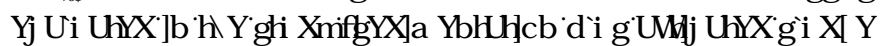
RULQMAP IWAQN GFDQMATH WAQG GDHDMRQUHDFYRUIDOMM Z KLFKDDFLOVDVAGRQQ [SKI VFDQUHP RYDQRI RRFI WWTYKH (D) RRQLM WHP VGGH RQWWDMAGIXEWDQMDQUHP RYDQDQG LQFWYDMRQRIRRFI WW DQLQFUHDMHLQGUP RYDQRI Cryptosporidium RRF। WWEI D

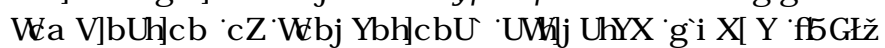

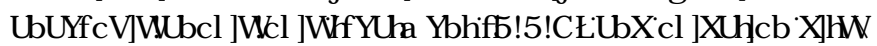

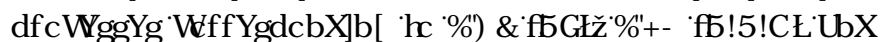

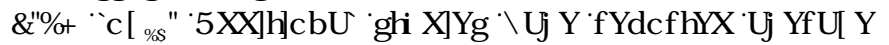

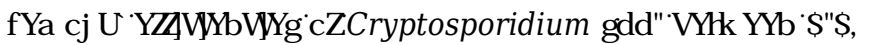
DQG

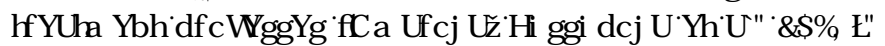
5RGUJ XH] D DQ] DQR [MVDQ

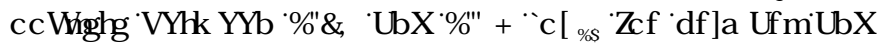

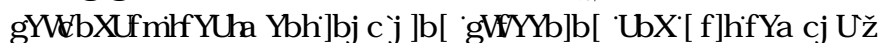
SUP DY LMHGP HQVDRQIDQDHREIF[G] HMRQIDQGMFFRGDY VHGP HQMDQRQT

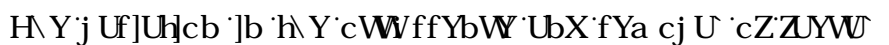
LQGLFDRUVTCryptosporidium RRF। WWTGiardia DQG HQMAJF YLXVHV [Z DVIQYHMY DVAG [Q]RXU[6Z HGLVK DMHFRQGDU

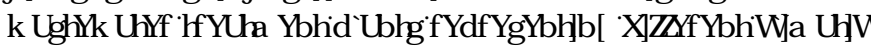
]RQHMIR WRRQTHDQ

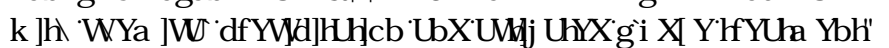
6RP HDDFLOMHMLFXGHGDGGWRQDQQLUR HQUHP RYDQDQG

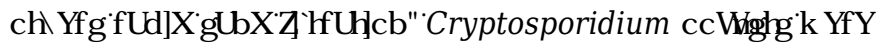

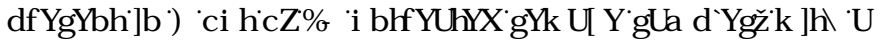

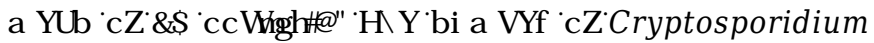

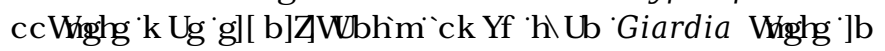

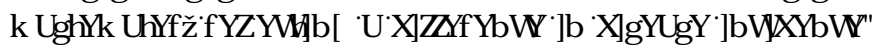

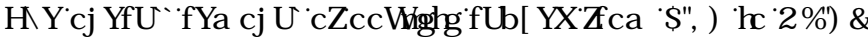

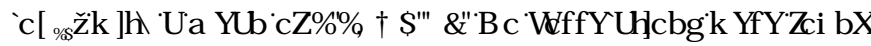
EHRHQCryptosporidium RRF WWDQGIDFDQLGFDMRUVDQG

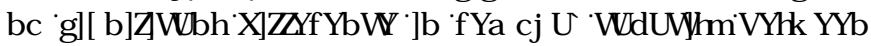
SQDWFRXQEHSLRYHQ

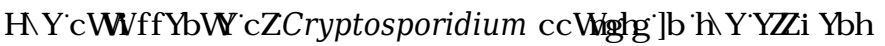

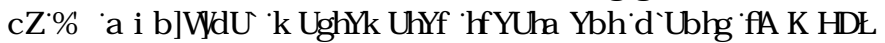

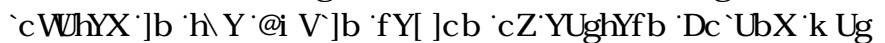

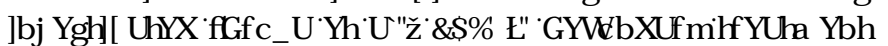
FRQMWAGRIDFUYDUAVIOG] HRUDFUYDUAVIOG HFRP ELQHG ZLVK,QFUHDVHG [UHP RYDQRI [QLWR HQDQG [SKRVSKRLRXV]

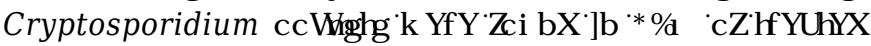

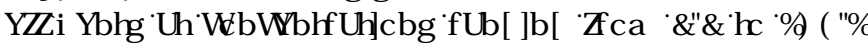

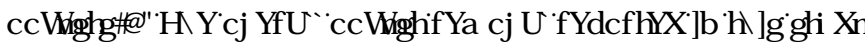

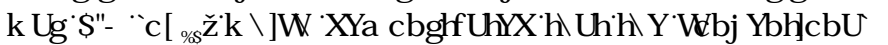

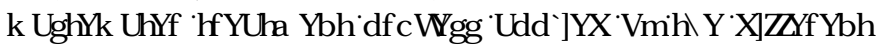
0 : 73[ZDVORVHIIFWYHIRUUP RYDQRIRRFI WW

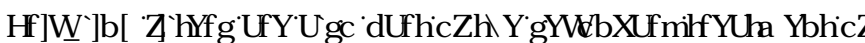

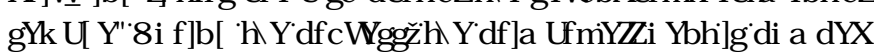

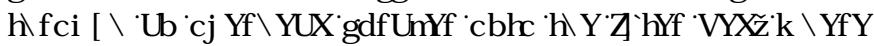
EDFYAUDDQGRUKHUP LFURY DQLP VIKDYHDRUP HGDELRI LP

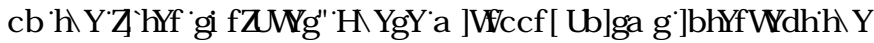
RY DQF P DMAUDQDVПWVMUFNON SDWVDQG [GHFRP SRVHПW

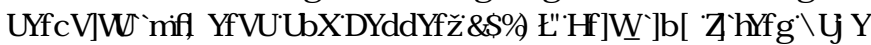

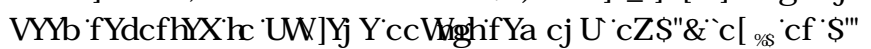
(R) 0 RURYHUIQR [GUIHUHQFHVRQRRFI WMHP RYDQ/KDYHEHHQ

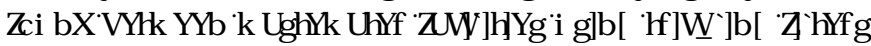

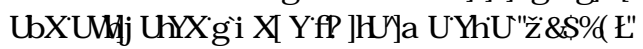

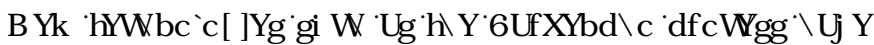

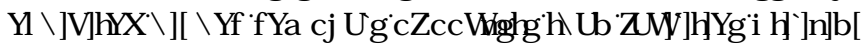

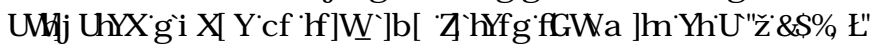
7KHपLYLWV HQ/DGHQSKR

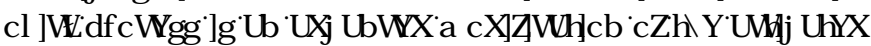

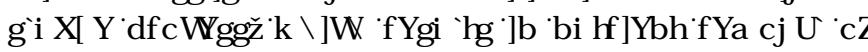
QLWR HQDDQG[SKRVSKRUXVGYDP IFURELDOSURFHMHVDQD P XOUMVI HEELRQI IFDQUHDFVRUTP/DUGHQSKR DM WAP VDUH

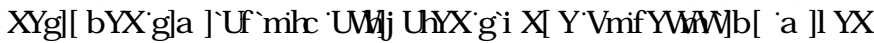

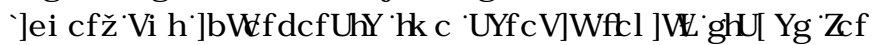

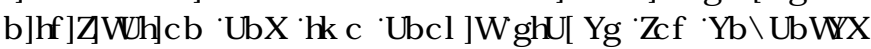

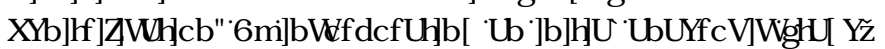
SKRVSKRUXVUHP RYDQIVTHQKDQFHGDVP IFUREHMUHODDHTYKH

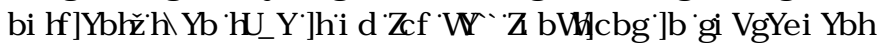
DHRELF[R[ IF[FRQGWRQV] RI [UP RYDOVRI Cryptosporidium FRUHSRQGQ [YR/m IRXQG[QRZ DMAZ DUAU[WHDVP HQWIDFLOWHN[XWO] LQ] [MKH

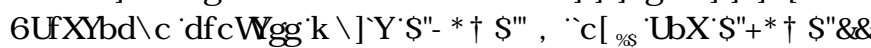

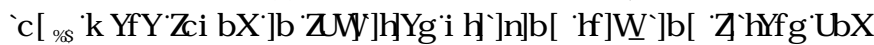

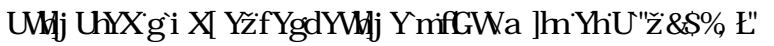

0 HP EUDQHELRUHDVRUM

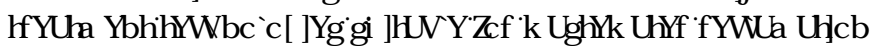

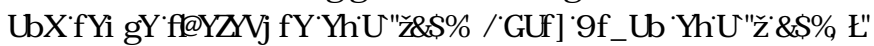

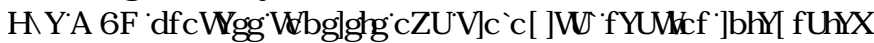

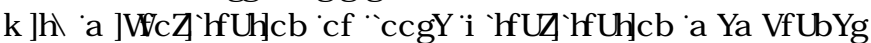

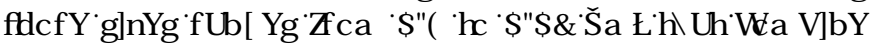

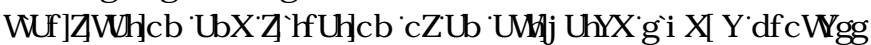

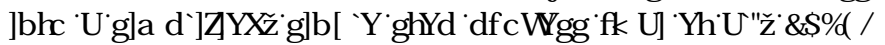

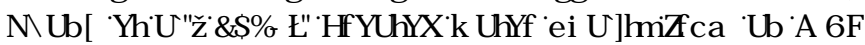




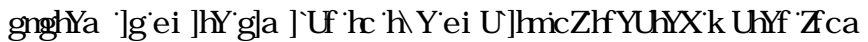

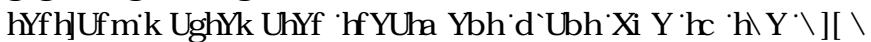

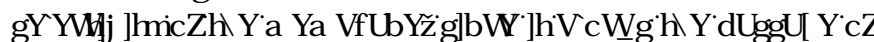

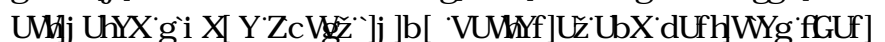

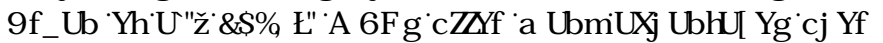

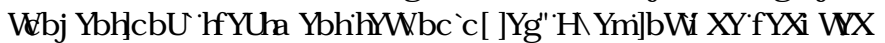

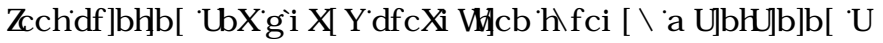

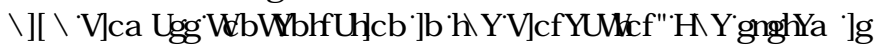

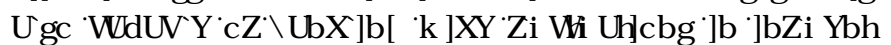

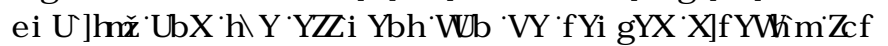

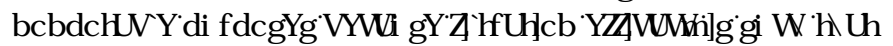

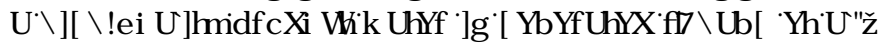

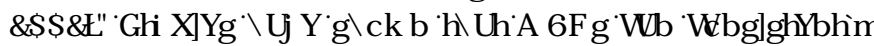
DFKIHYH[HILFLHQVIHP RYDOVRN [VXVSHOGHG[VRQGISLRVR] RD]

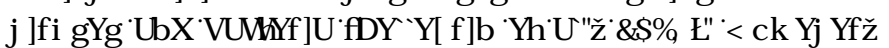
SDUKRJ HQJUP RYDQHI LFLHQFLMLLD \%5 VIKDYHEEHQUUHSRUMG RQQ]RUGiardia Z LIK[GI DQM

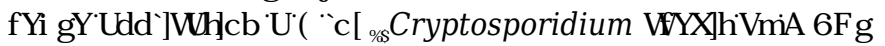

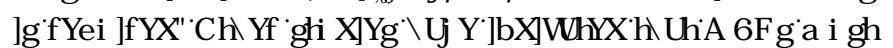

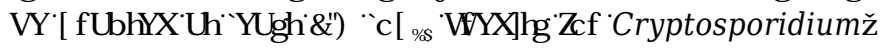

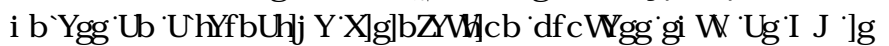

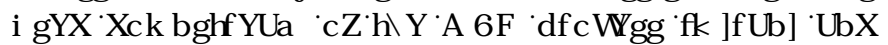
-DFDg HRRा

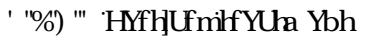

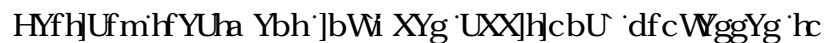
IXUKYHZHP RYH[SDMKRJ HQVDHMGXDQVXVSHQGHGLVQGVDQG

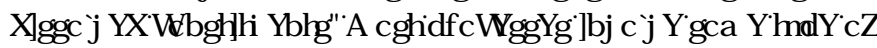

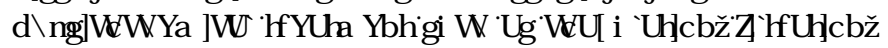
DFUYDUAGFFDERQDDVRLSURQIRI RY DQIFVTHYHUMHRVP RUV

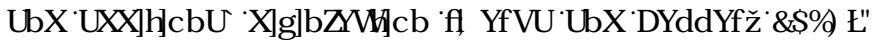
\$P RQ [DIZ LGHDDI HRI [MAUWDU [DQG[DGYDQFHG[MHDWF HOW

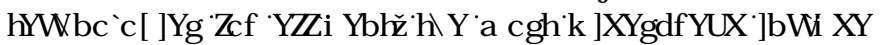

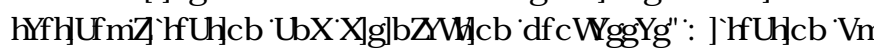

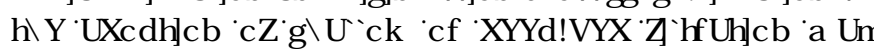

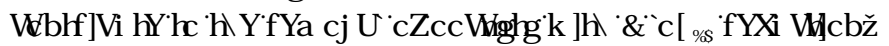

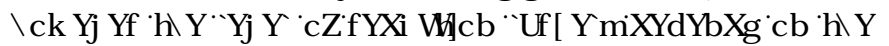

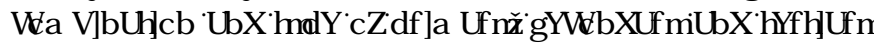
WHDVP HQVVDSSCHG[DV[GHP RQWWDUAGLQ[SUHYRXVDWXGLHV

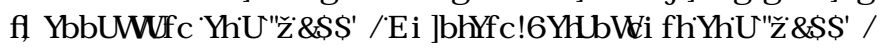

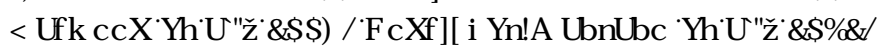

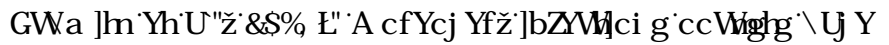

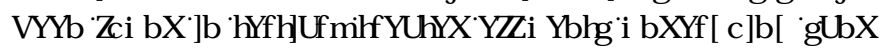

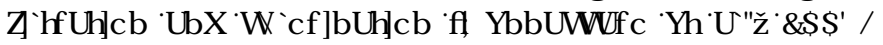

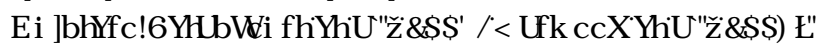

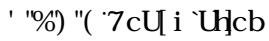

\&RD XODMRQDQG] RFFX(DMRQ]VIDQ]P SRUDDVWMASTQ

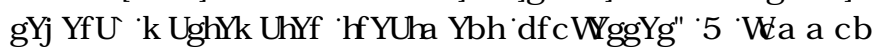
H DP SOILVFKHP LFDQSKRVSKRLXVUIP RYDQDQGDORUKHUR

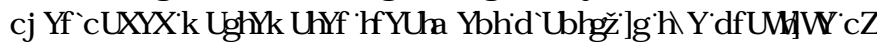
FKHP LFDQQ [HQKDQFLO] [SUP DU [NHDVP HQWDMR ]UHGXFH

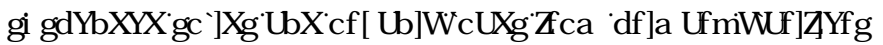
IKWSV

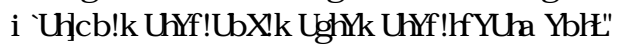

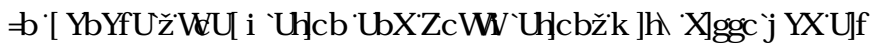

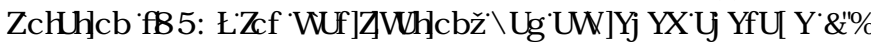

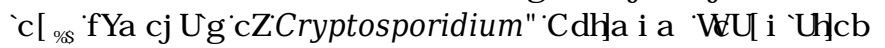

FRQGLURQVMH +P HGLIREF] RYHQHGEE [YXUELGLWIDQGQDDXXDO

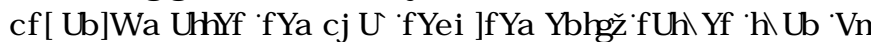

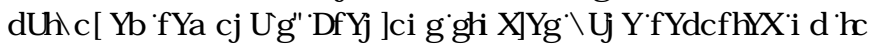

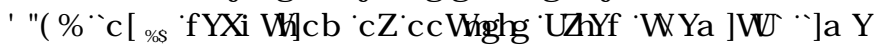
WHDVP HQWE KHQSSUFFHGGEI [SUP DU [WA-DUF HQMIDFUYDUAG

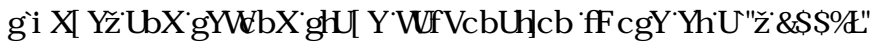

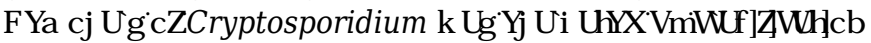

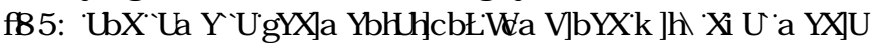

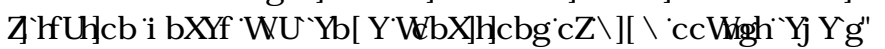

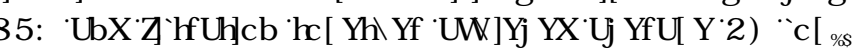

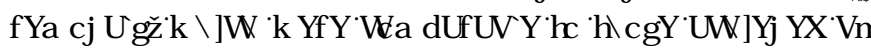

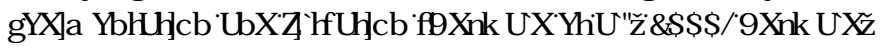
प्रापा

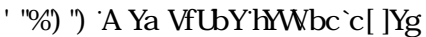

0 HP ELDQH[SLRFHMHNGKDYHEFHHQZZLGHQ DSSSOHGRQ

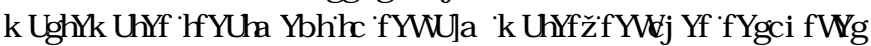
RUTHYHQ[SURGXFH[HQHY \एD HP EUDQH[SURFHMHVIFDQLEH GUYHQ EFI [SUHWXUH [IP LFURI LOWDWRQ] XXOUDI LOWDWRQ]

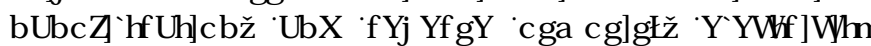

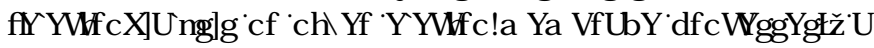
FRQFHQWDURQ] UDGHOWW RUZ DUG RVP RVYIRUD[MKHUP DO J UDGHQWWP HP EUDQH [GLWWCOWRQ IDQG $\square$ P HP EUDQH

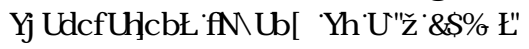

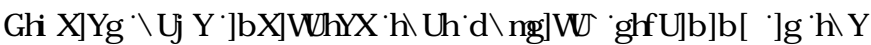
SUP DU P HFKDQLVP [RI [DFURQ]RU]HP RYDQRI [SLRVR] RDQ RRF WWIURP [MKH] HHG[ZDMAUGXUQ] IP IFURI LOUDMRQ/DQG

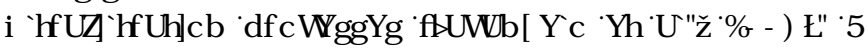
Z DWHZ DUAULMAUUDU IWHDVVP HQWEDVHG RQ PP HP EUDQH XONDI LONDWRQ IHG \&Z LVK IMHFRQGDU INHDMAG P XQLFLDO

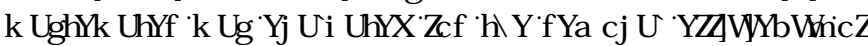
Cryptosporidium RRF WWW/ RQY URHMDQ

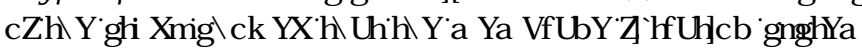
Z DV [XVHIXOG RU]HP RYDORI [Cryptosporidium ]URP

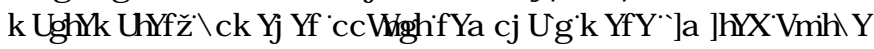

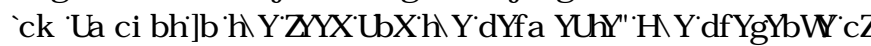

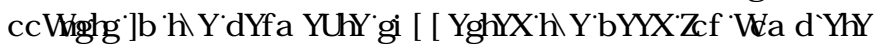

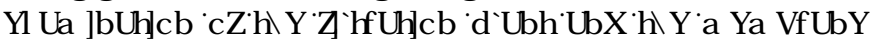

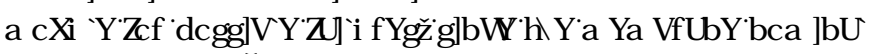

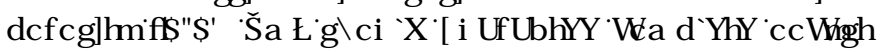
UP RYDQ

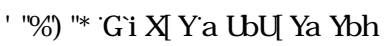

9DURXVLMHDVP HQW[DUH[XVHG]VR]UHP RYHLZDMAUDDQ

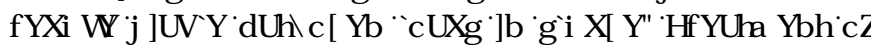

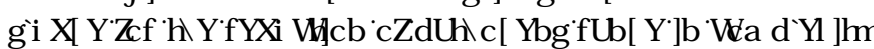
DQG P DI IOFXGHLDU[GU LO] IFRP SRWL] IDPHUREIF RU DQDHUREIF [GL HMRQIDQG[YDURXVCVKXHUP DQDQGFKHP IFDO

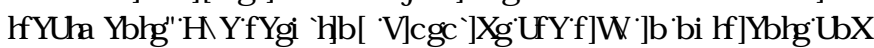

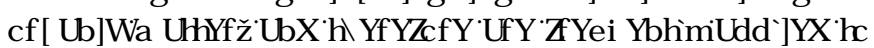

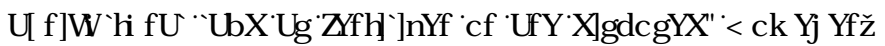

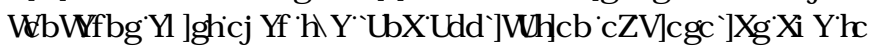
WKHSRUAQWDQUSUHDGRI [SDMKRJ HQVDQGRUKHUFRQMDP LQDQW]

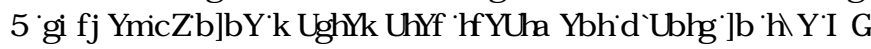

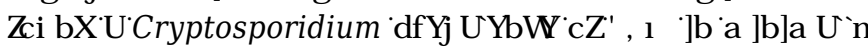
WHDUAG \&OW[\%[ELRVRQGVIZLIKLFRQFHOWDURQV]DO LQ]

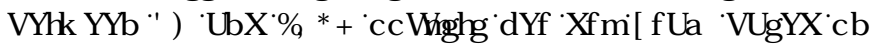

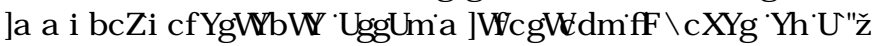

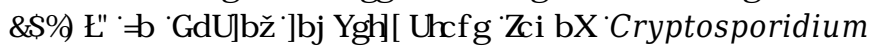




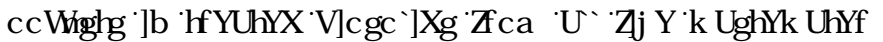
WHDVP HQWVSDQWLWXGHGIZ LIK DYHUD HFRQFHQWDMRQV

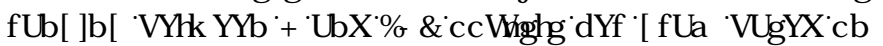

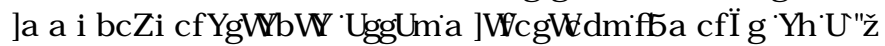

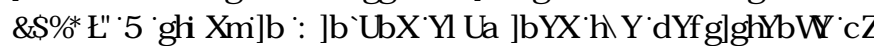

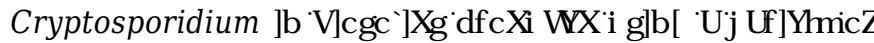

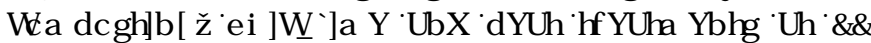

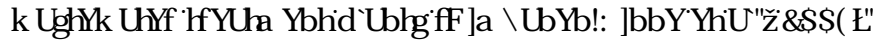

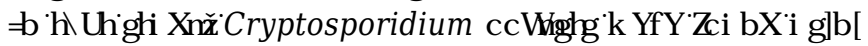

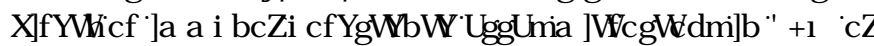

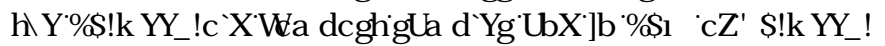
R@LFRP SRWIMDP SOM

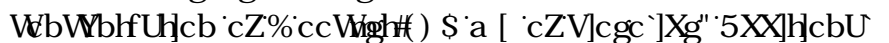
WXGHMKDYH[GP RQWDDAG]HGXFWRQNRI Cryptosporidium

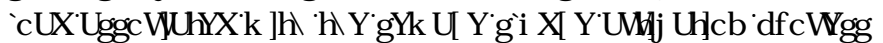

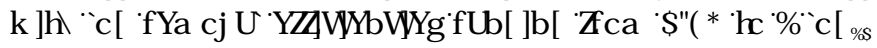

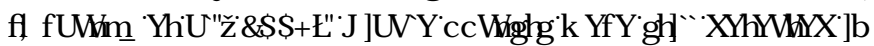

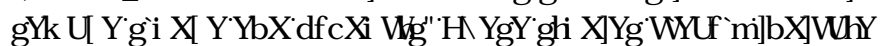

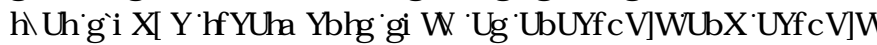
GL HMRQ][QP H[MDELQ] DURQ[DQG KHDWGU LQ] [GR [QRW HQP LQDVA [Cryptosporidium RRFI WWIQRP SRWL] [RI

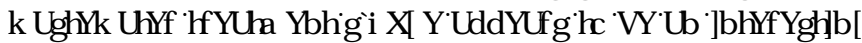

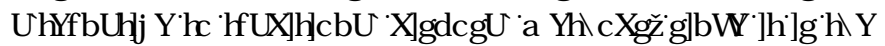
RQQ [MDELQ] DMRQ [NHDVP HQWFDSDEO1 [RI [HQP LQDMQ]

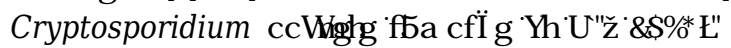

\subsection{Disinfection}

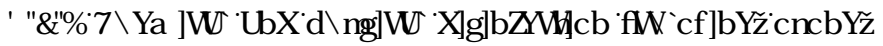
XQWDIROMUDGDMRQIIDGYDOFHGR[ LGDMRQSLRFHMHM]

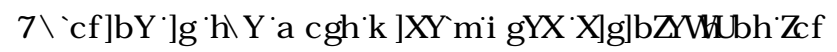
P XQFLSDQZDWAZ DUALEFFDXVIVUGHMRI VP IFURRY DQLYP V

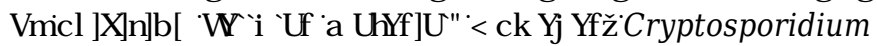
RRFI WW

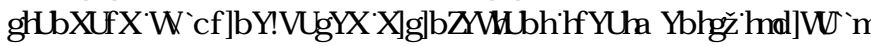

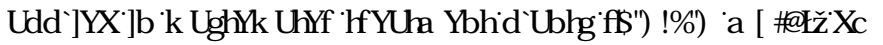

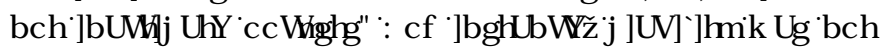

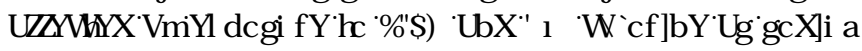

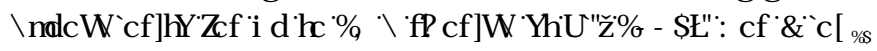

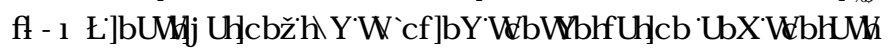

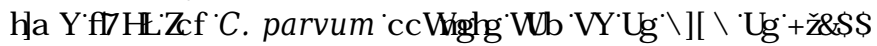

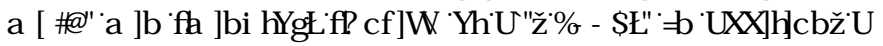

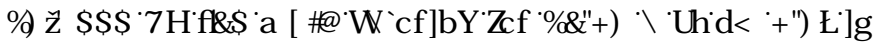
UHTXUHG]RLLQDFUצDMg [Cryptosporidium LQSRRQW6XSSHN

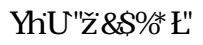

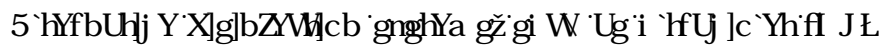

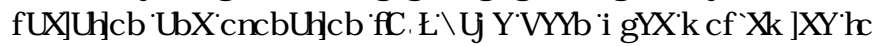

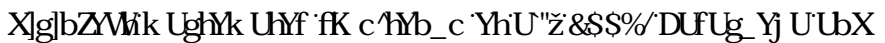
* LDKDP 皿

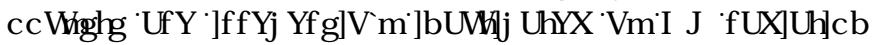
[5RFKHCOHMDQ]
RRFI WWIKDVEHHQGGH RQWDDAGDDY HUP IFLGDQB 9 [GRVHN

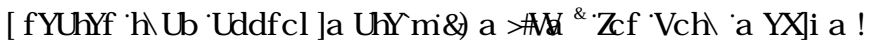

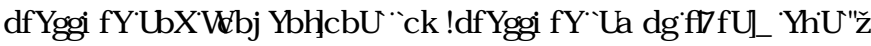

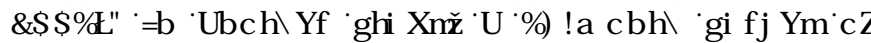
Cryptosporidium RRF WURFFXUHQFH[ZDVFRQGXFUAGDMUAQ 86 [ZDWHZ DUAULWHDWA HQWES DOWWDQG Cryptosporidium

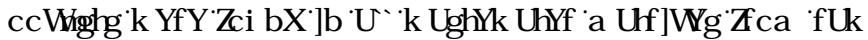

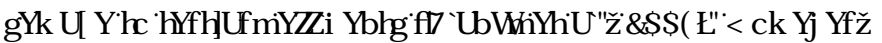

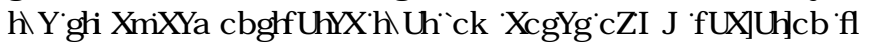

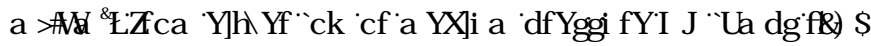

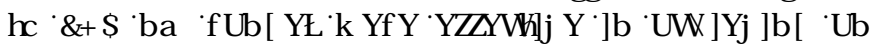

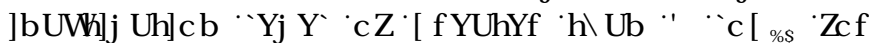

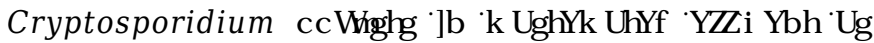

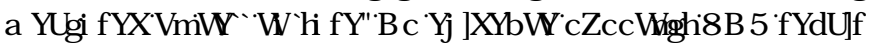

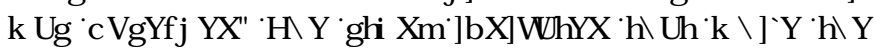
RFFXUHQFHRI [Cryptosporidium RRF WWLZDVFRP P RQRQ

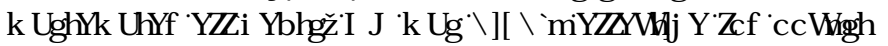
LQDFUYDURQILTZ DMAZ DMAU]

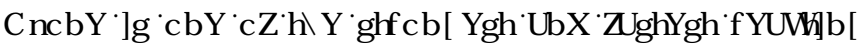

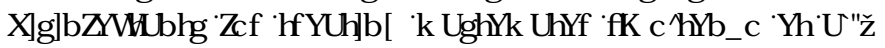

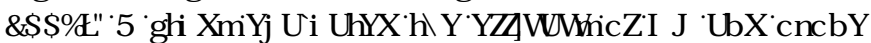
WHDWF HQVM RLUHGXFWRQRI Cryptosporidium LQ\&62 VTPKH

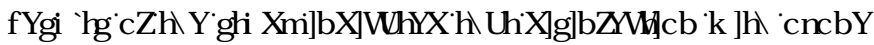

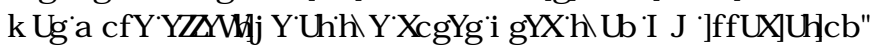

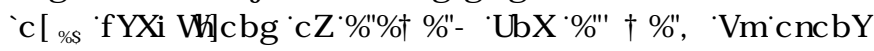

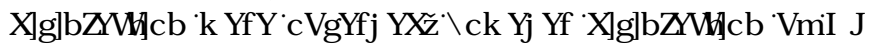

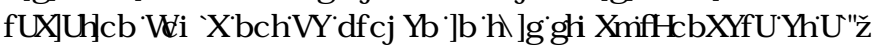
प्राप्या

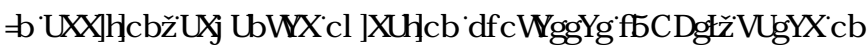

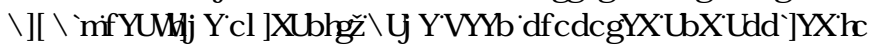

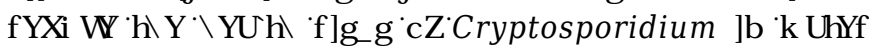

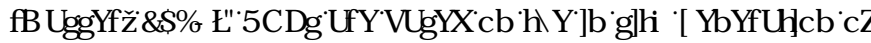
WWRO [R[ LGDQW]RUIMKHR[ LGDMRQRIIRU DQLF[FRP SRXQGY]

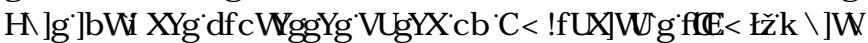

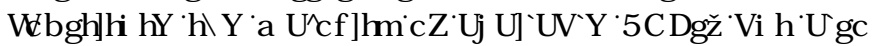
SURFHMHMEDMHGRQRMYHUR] LG] LQ [VSHFHMIDYRUQ [VXФDUA

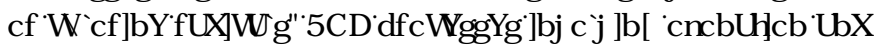
8 9]DGDMRQDUHDQHDG \& HCOHMEEOKHGDQGRSHDUAGDW

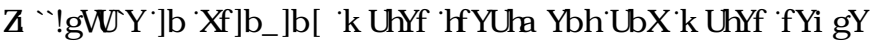

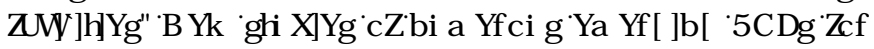

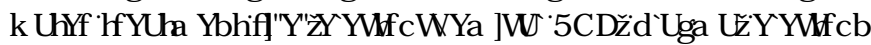
EHDP IIXQUDVXQQRUP IFLRZ DYHEDMHQ\$2 3VIDUHFRQMEQN

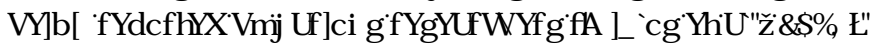

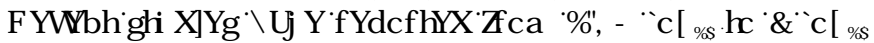
LQDFUYDURQRI [Cryptosporidium SDDMUAEEI [SKRMRFDUDQVVV

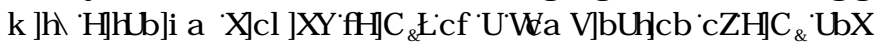

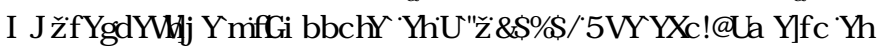
DQIIII)

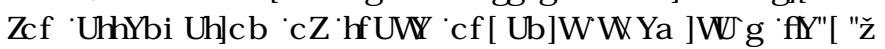
SKDUP DFHXWFDQMTFRQVXP HU[SURGXFWDDQG GQGXWUDO

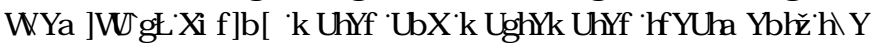

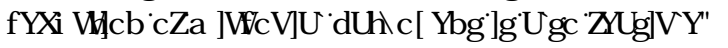




\section{References}

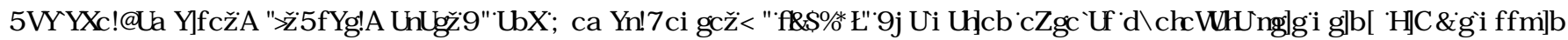

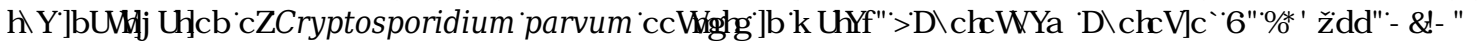

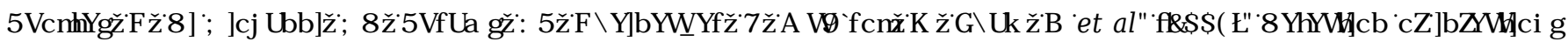

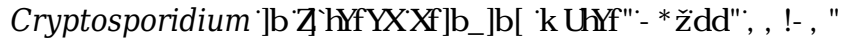

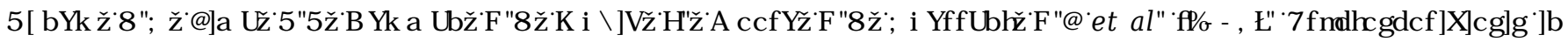

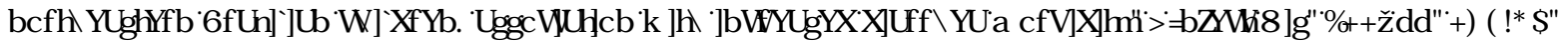

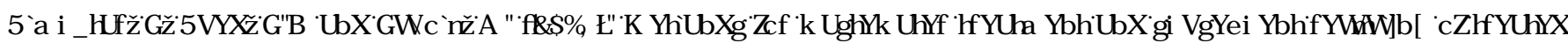

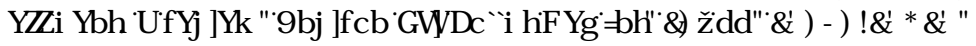

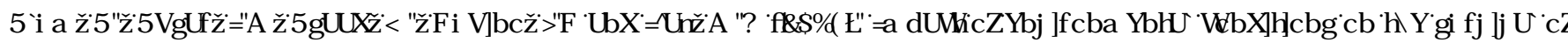

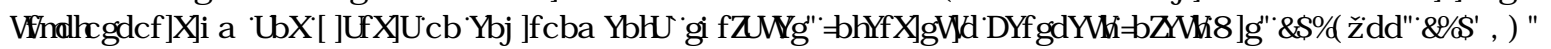

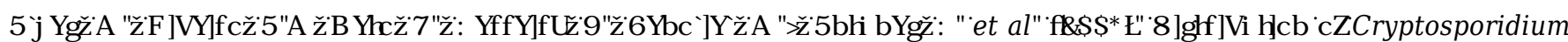

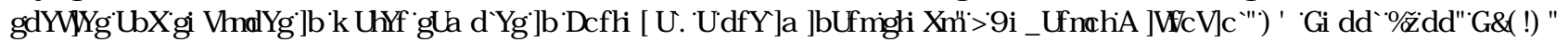

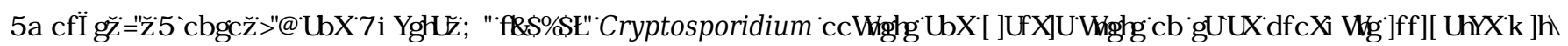

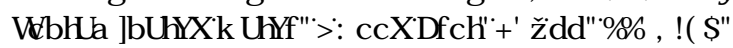

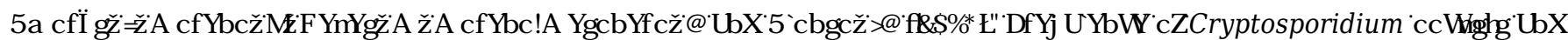

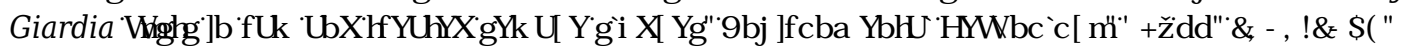

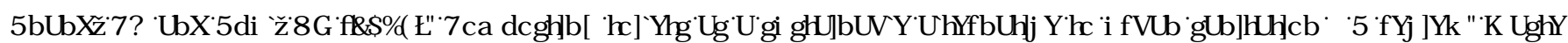

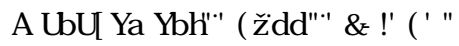

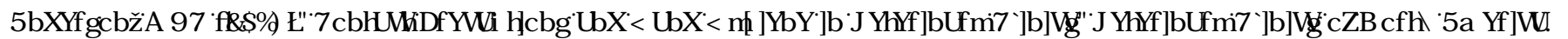
6P DQ\$QP DQBLDFUFH

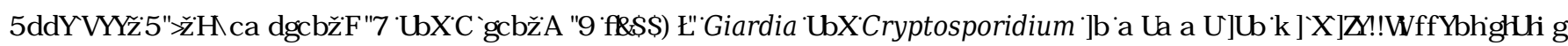

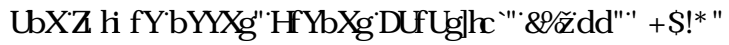

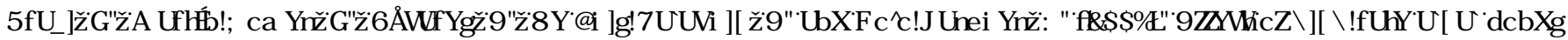

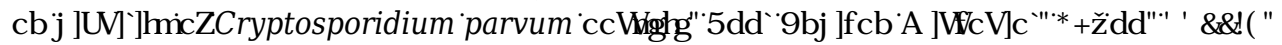

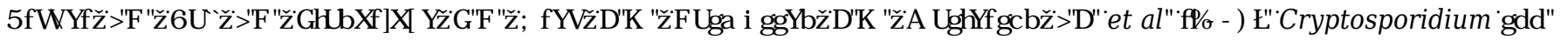

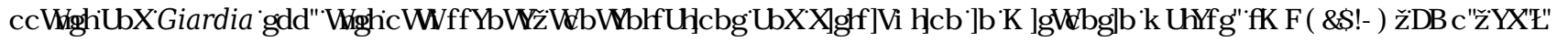

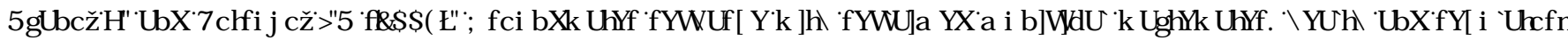

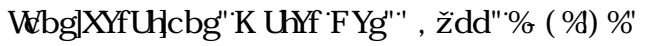

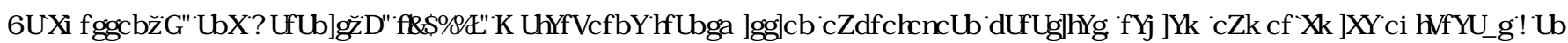

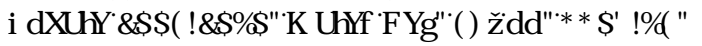

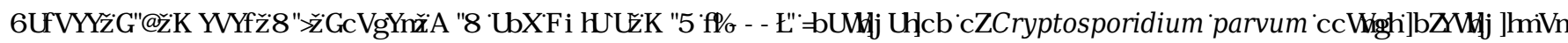

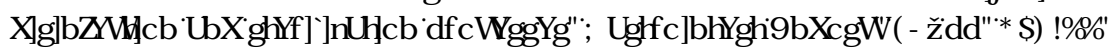

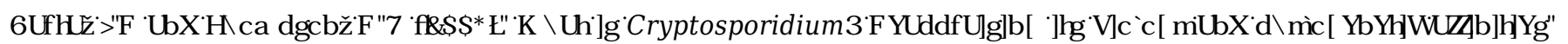

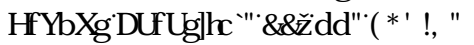

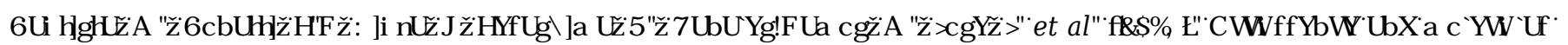

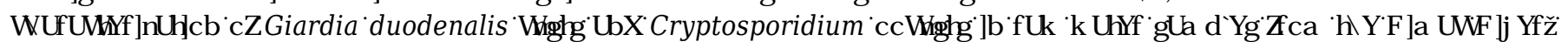

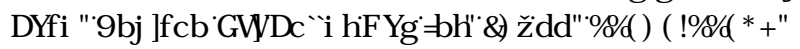




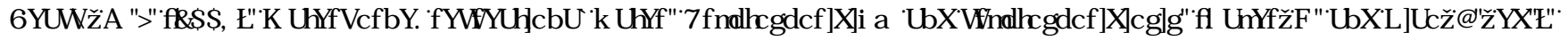

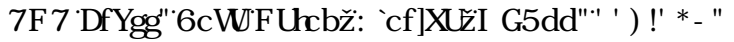

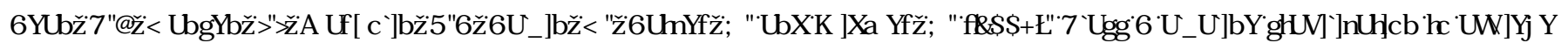

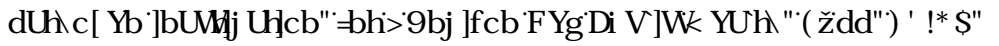

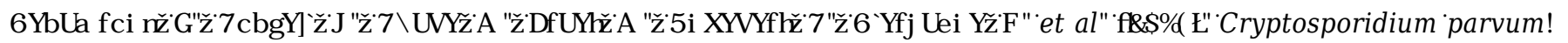

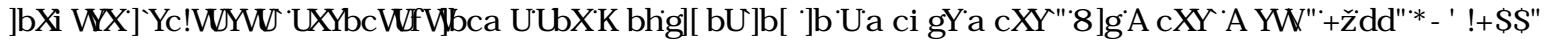

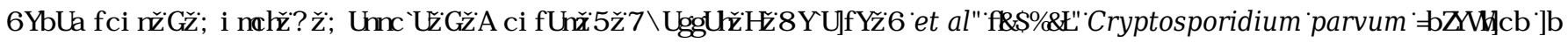

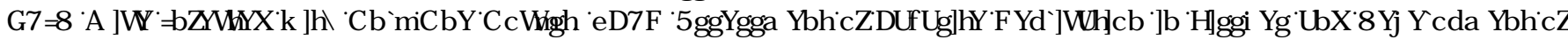

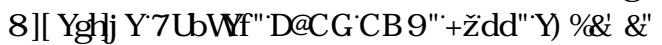

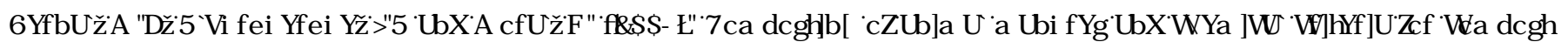

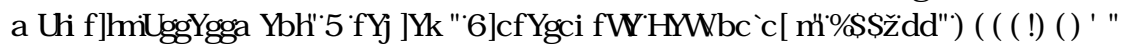

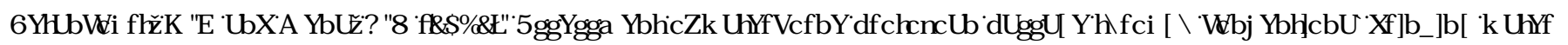

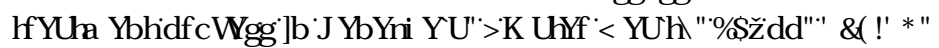

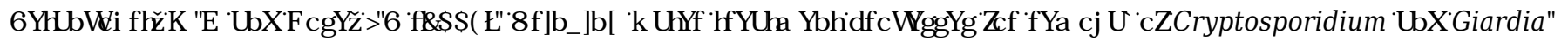

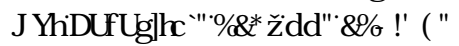

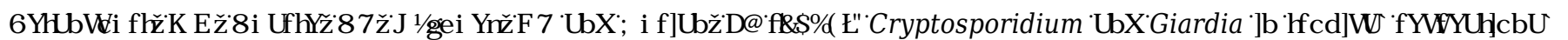

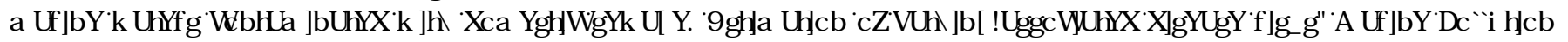
\%रComQ

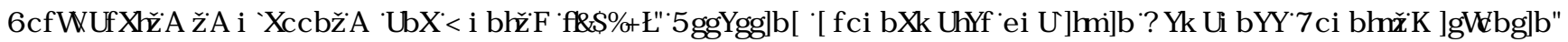

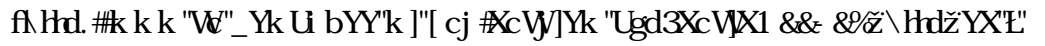

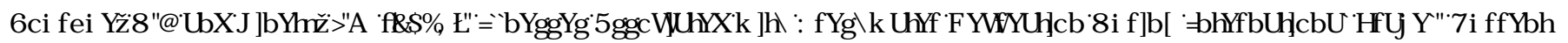

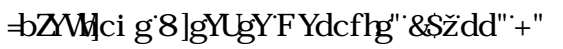

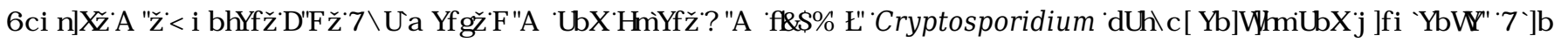

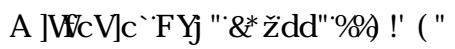

\%RX] LG!

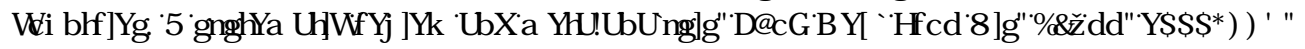

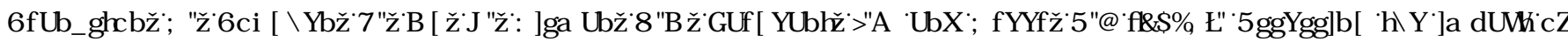

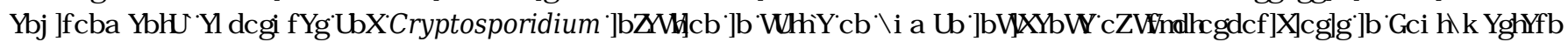

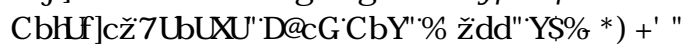

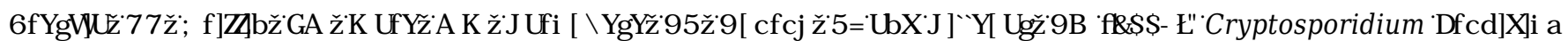

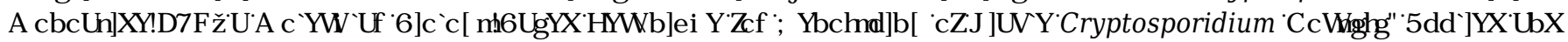

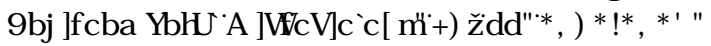

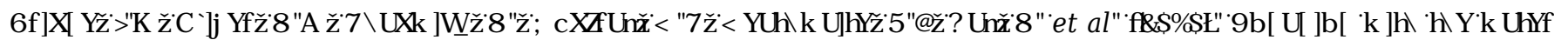

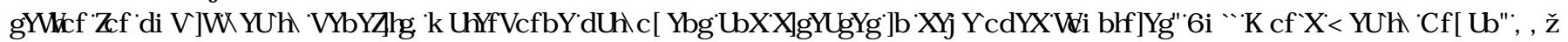
SS

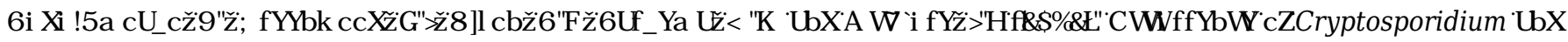

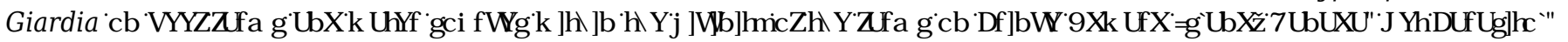

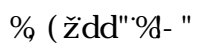

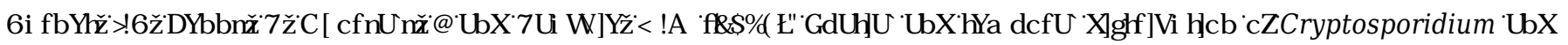

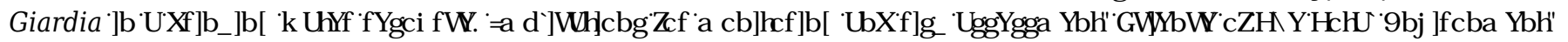

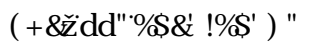




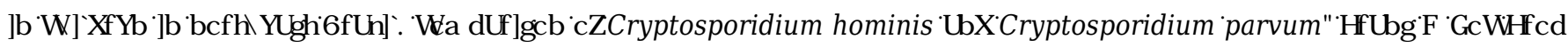

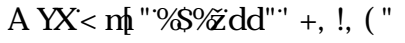

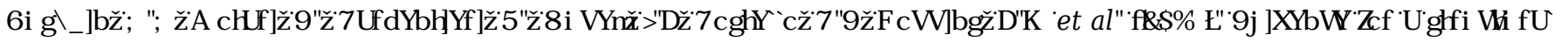

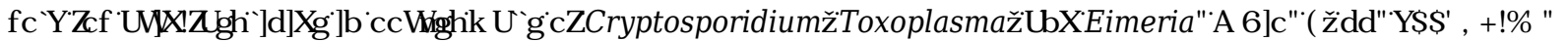

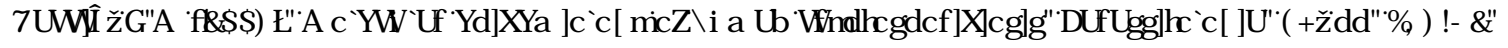

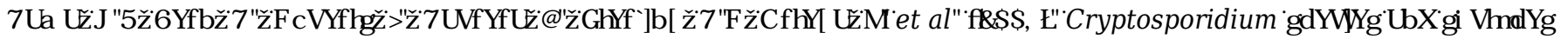

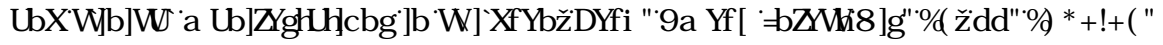

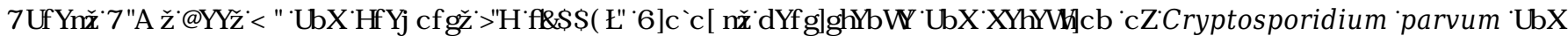
Cryptosporidium hominis RRF WV: DMA

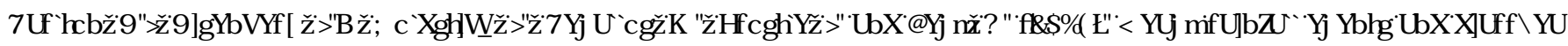

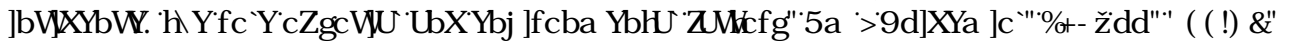

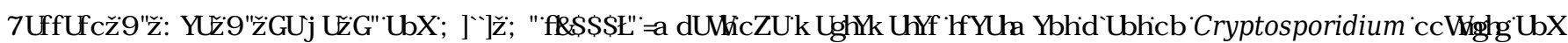

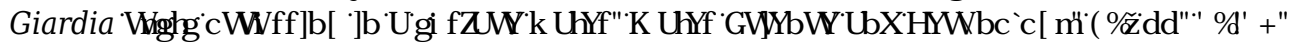

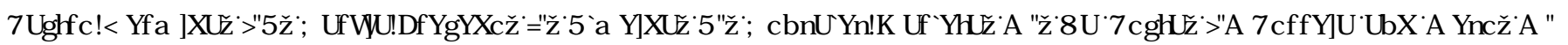

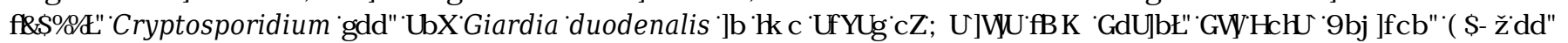
परापाला

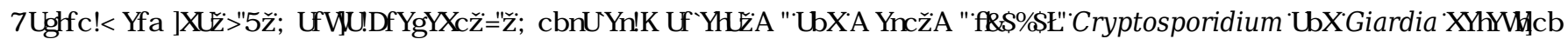

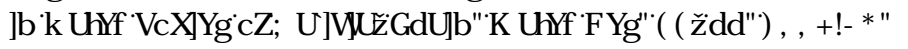

\&DYDOHU6P LMXIP

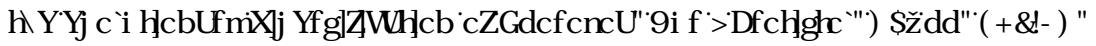

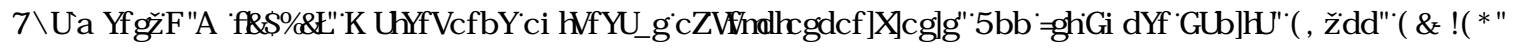

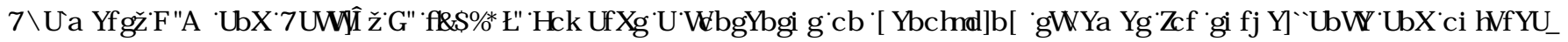
LYHMWy DMRQNRE Cryptosporidium

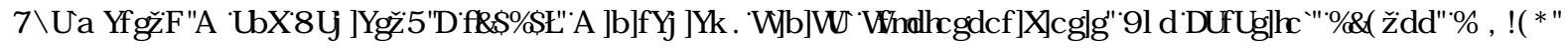

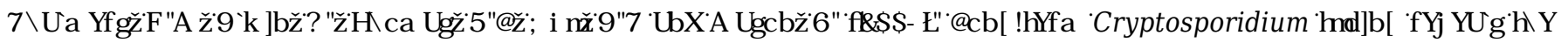

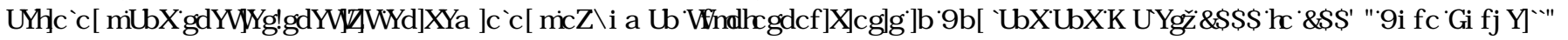
प्राप

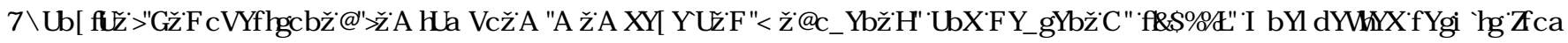

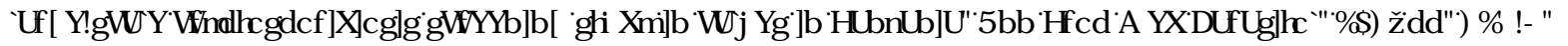

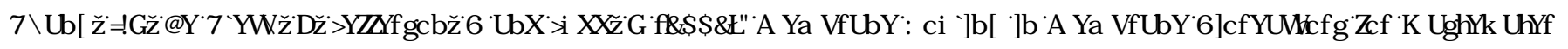

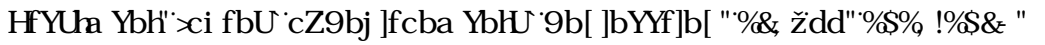

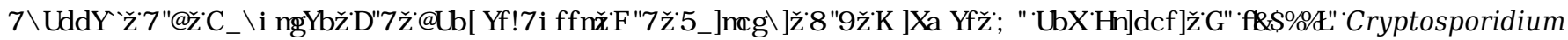

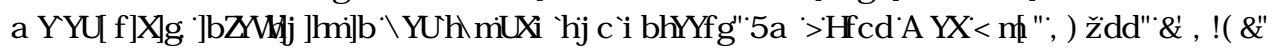

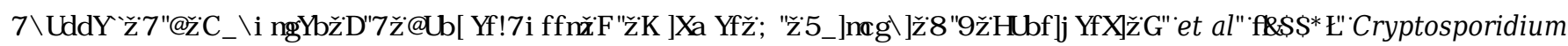

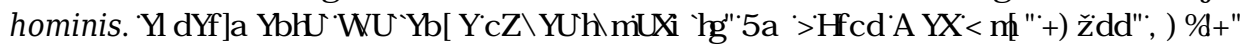

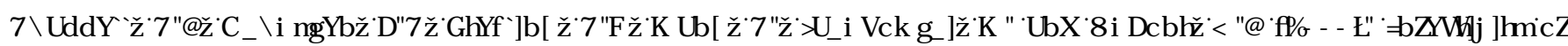

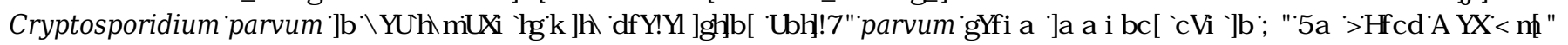

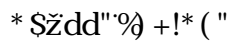




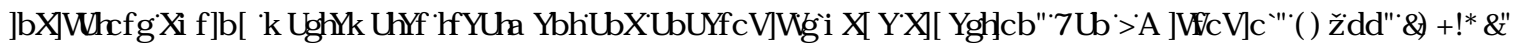

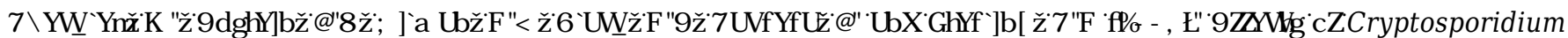

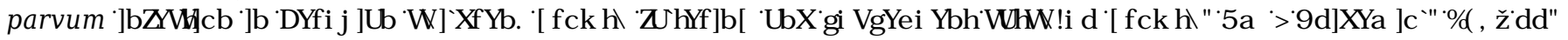

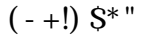

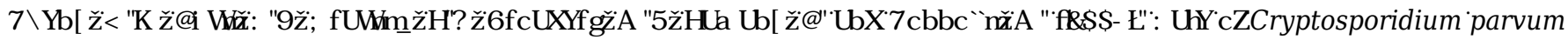

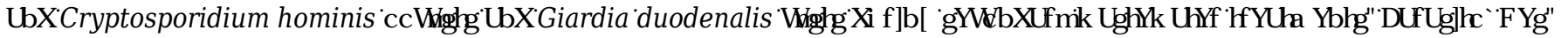

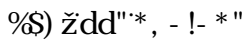

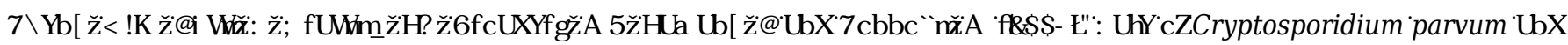

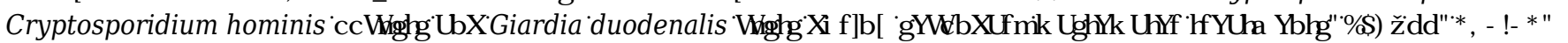

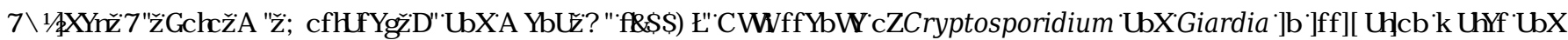

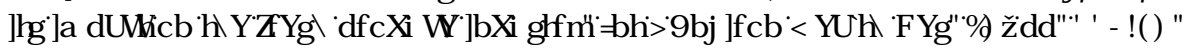

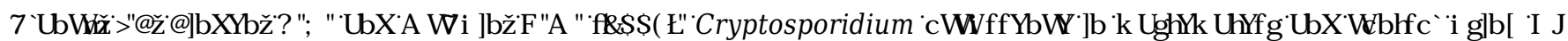

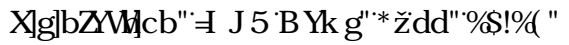

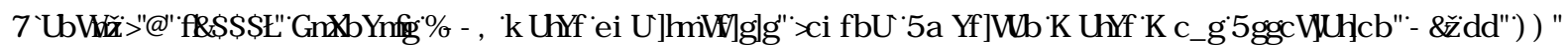

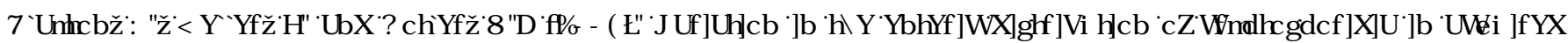

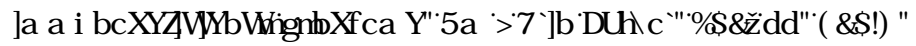

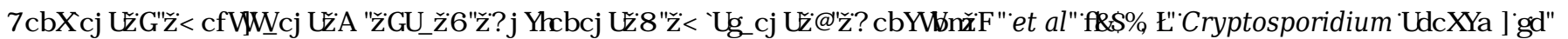

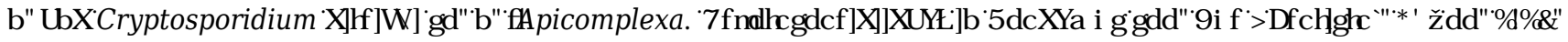

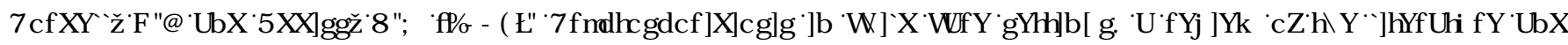

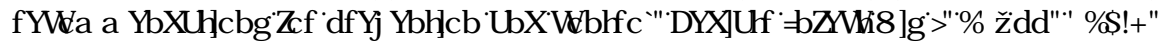

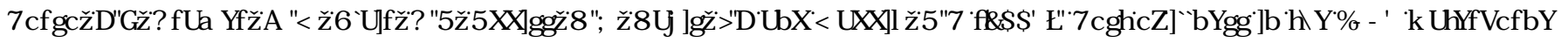

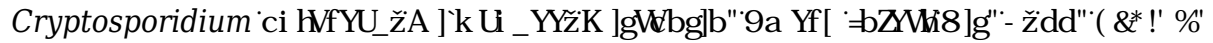

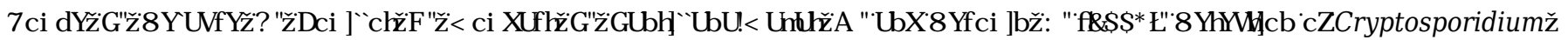

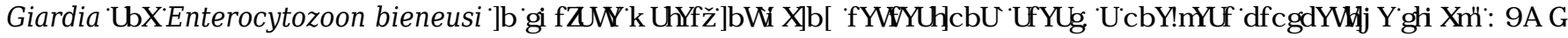
,P P XQR@D HGD IFUREIRQm]

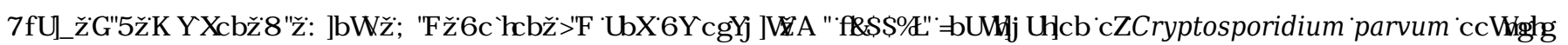

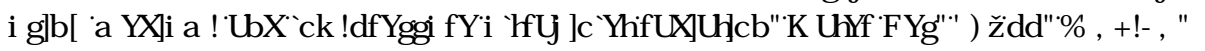

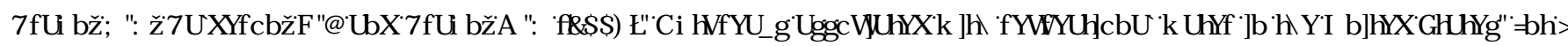

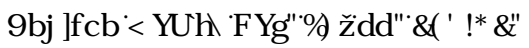

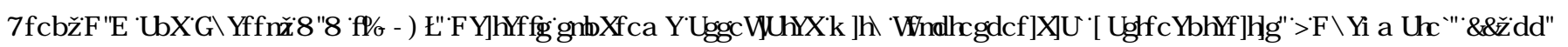

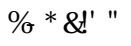

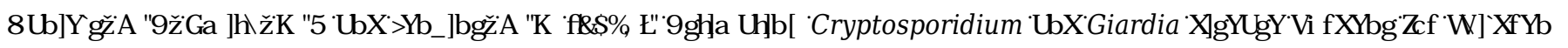

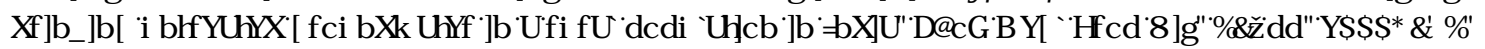

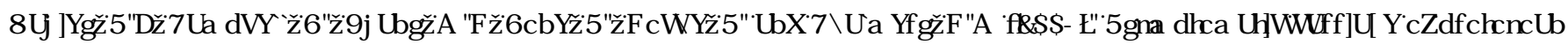

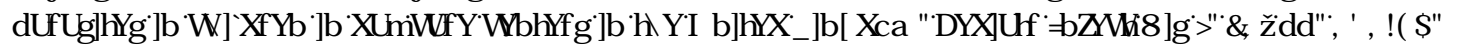

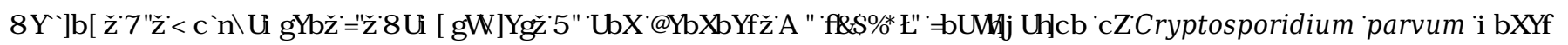

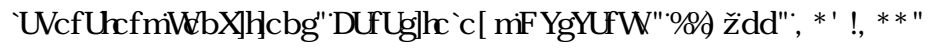

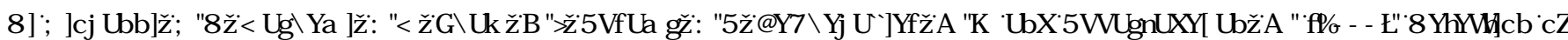

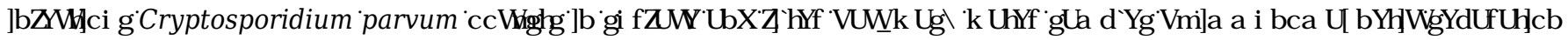

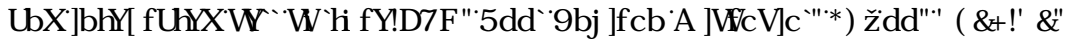




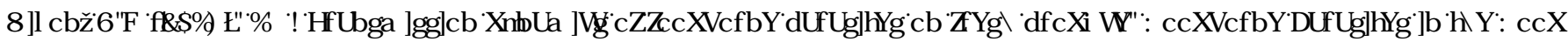

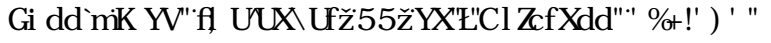

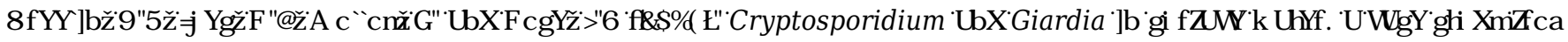

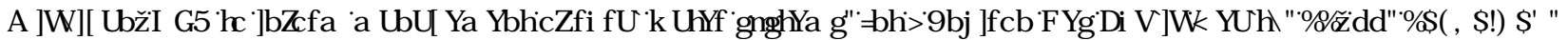

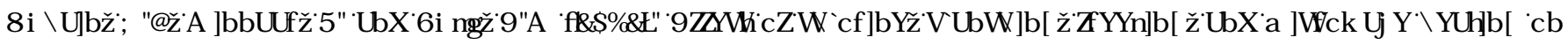

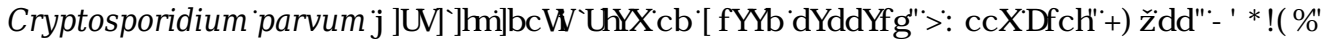

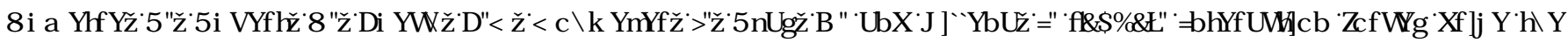

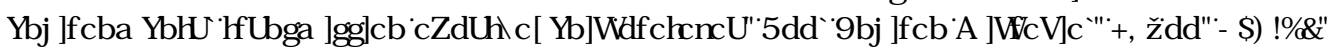

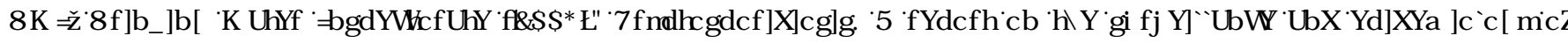

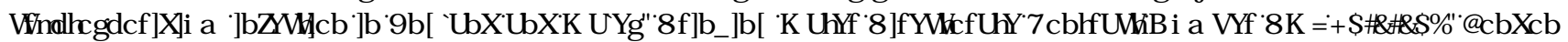

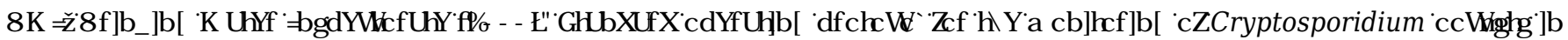

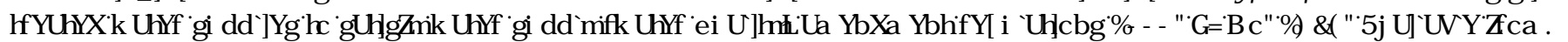

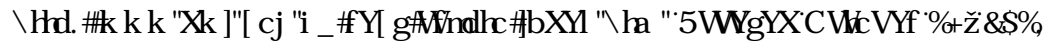

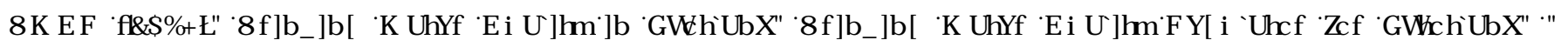

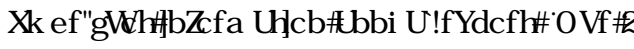

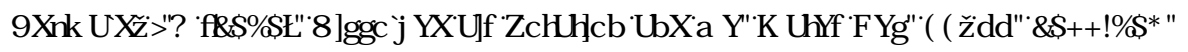

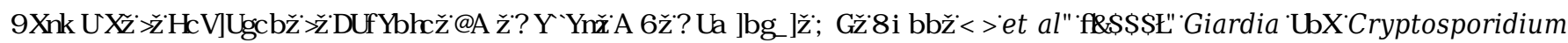

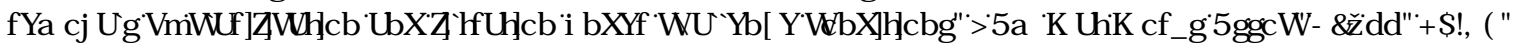

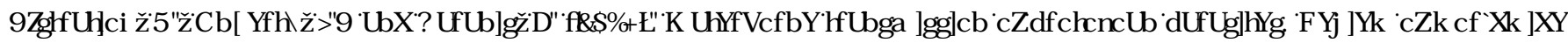

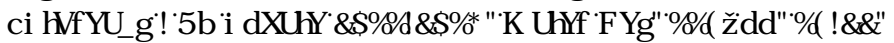

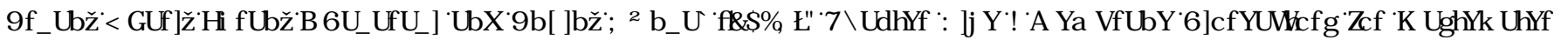

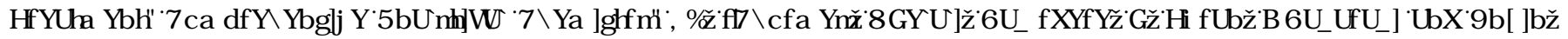

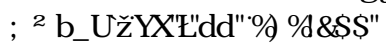

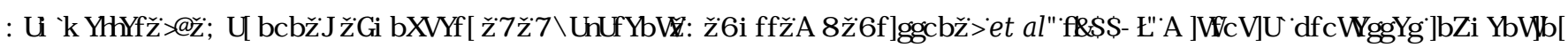

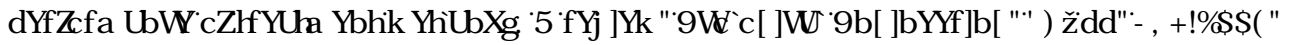

) D HUा5

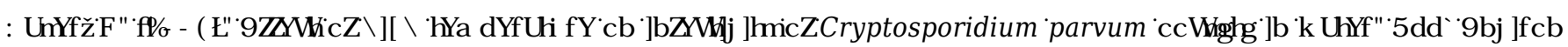

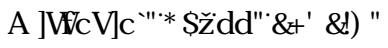

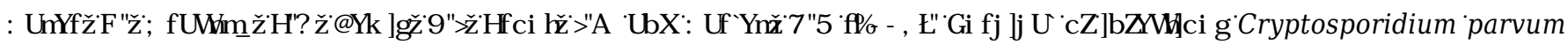

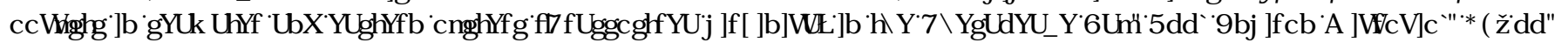

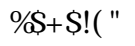

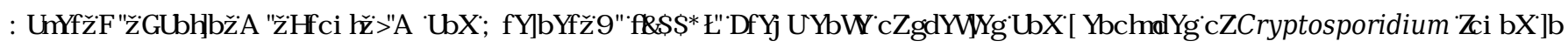

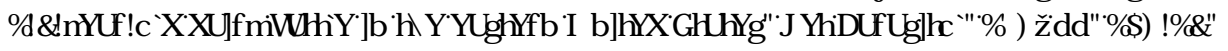

) D HUT

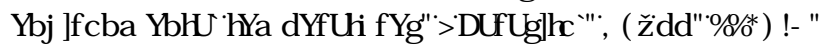

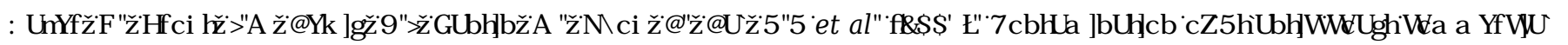

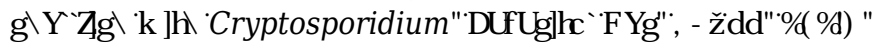

) D HUाb

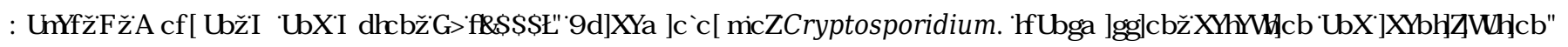




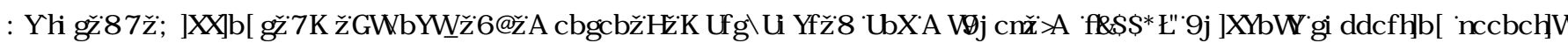

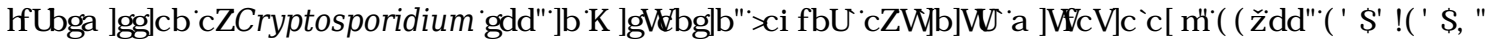

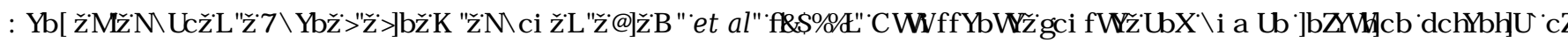

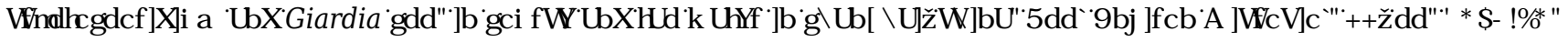

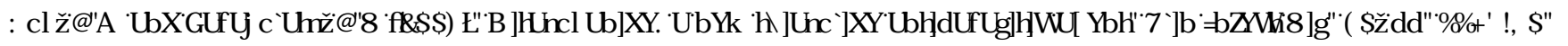

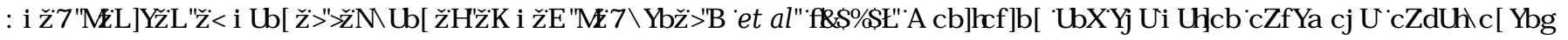

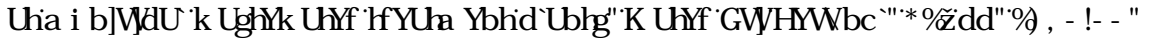

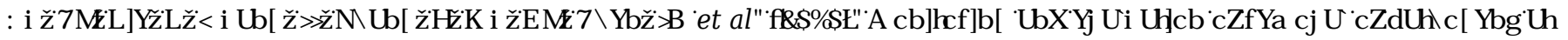

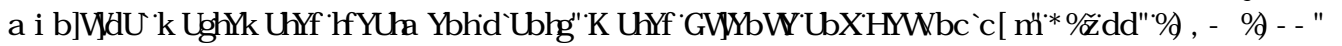

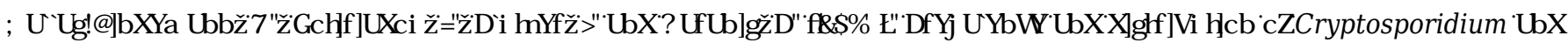

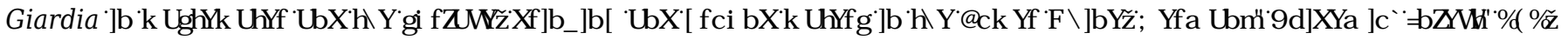

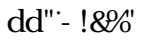

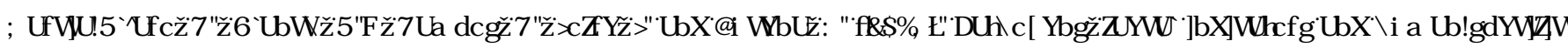

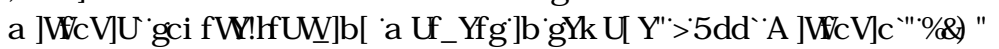

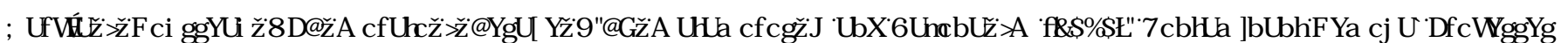

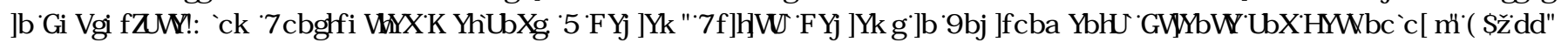

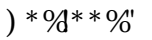

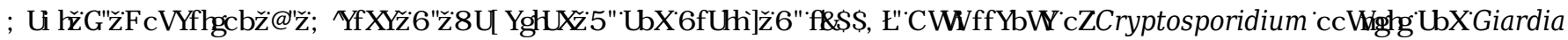

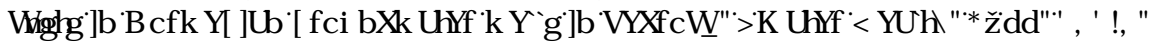

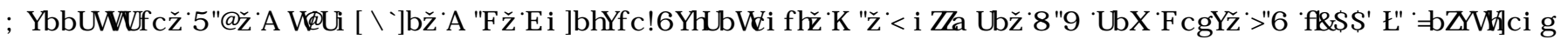

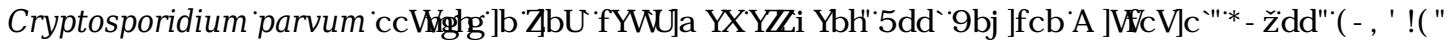

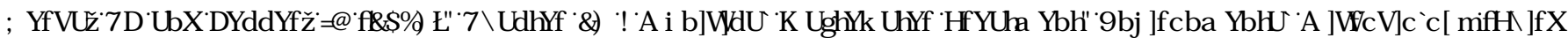
( GURQ

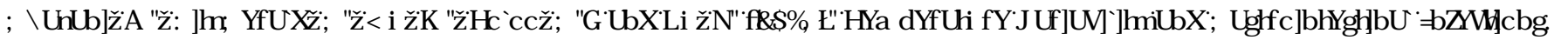

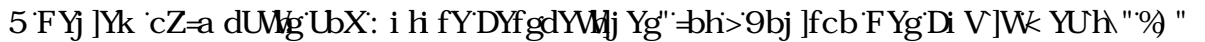

* LO DVSHRW\$

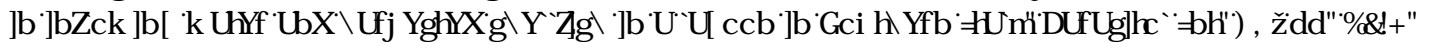

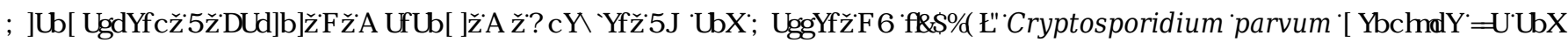

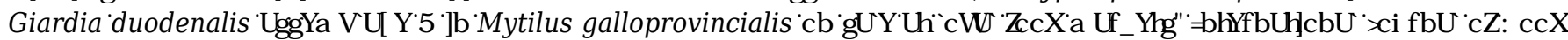

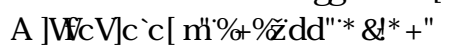

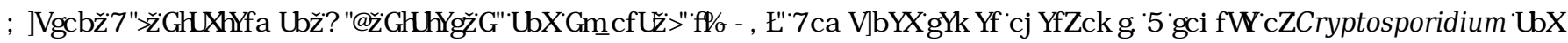

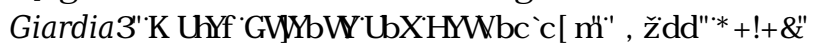

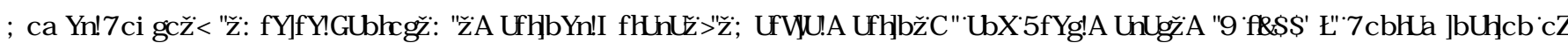

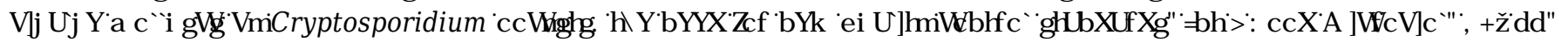

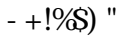

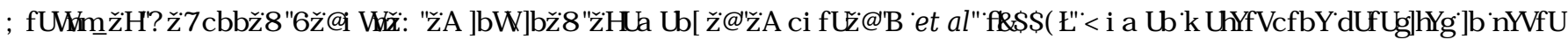

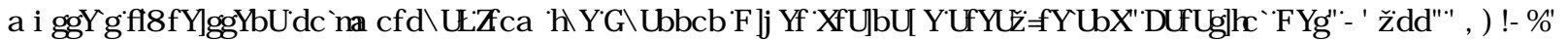

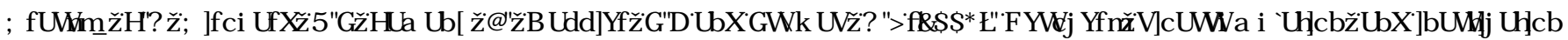

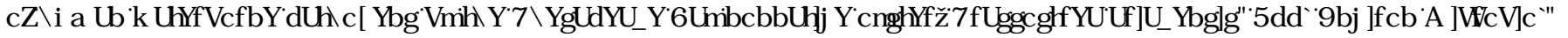




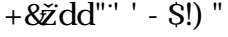

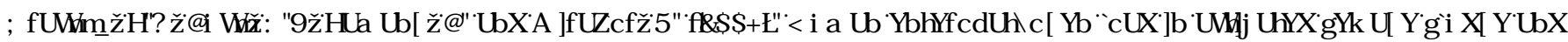

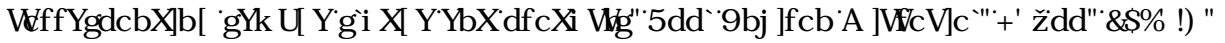

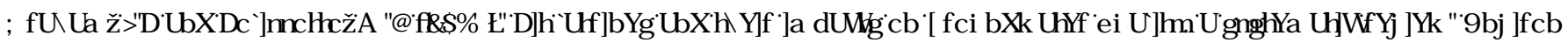
+HDOKEBHUSHFVM]

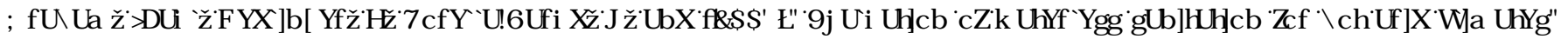

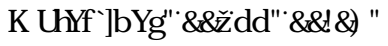

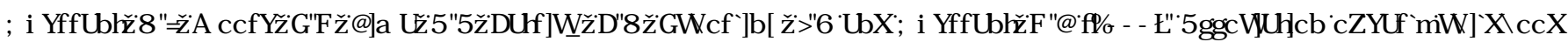

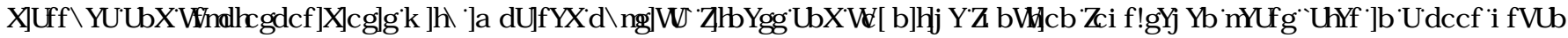

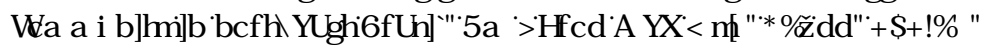

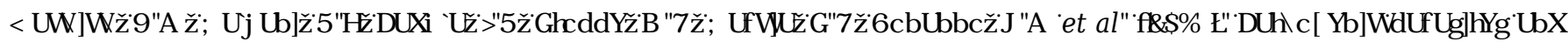

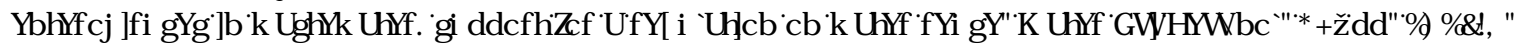

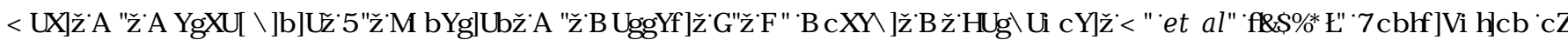

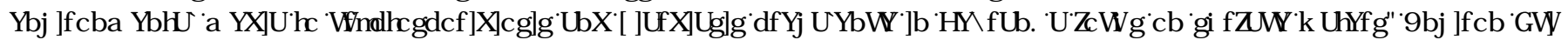

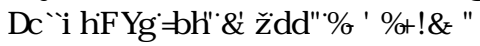

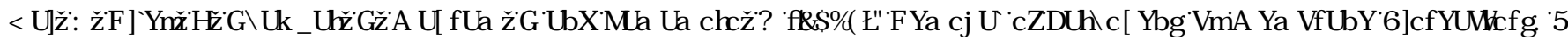

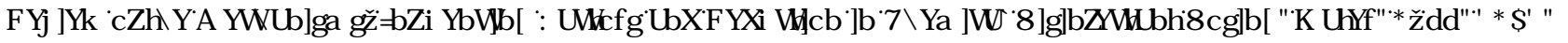

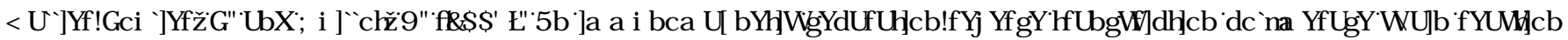

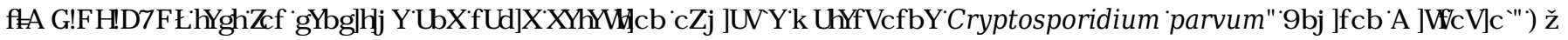
SS

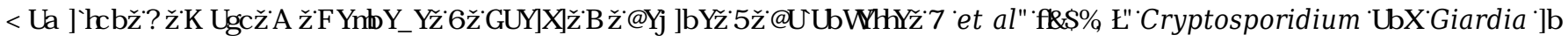
ZDUAZDMADQGIXUDFHZDMATHOYLRQP HQW

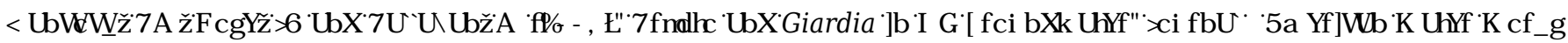

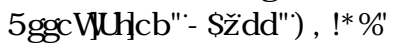

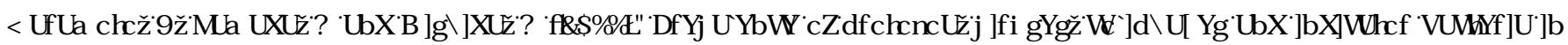

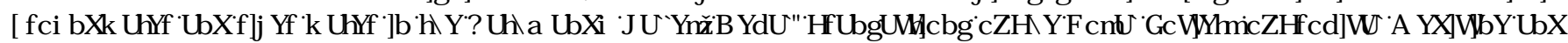
$+\backslash \mathrm{J}$ HQH

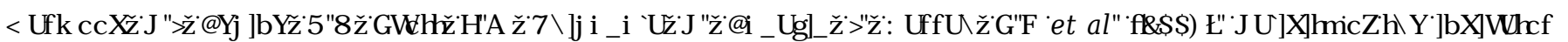

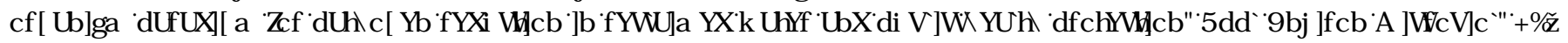
SS

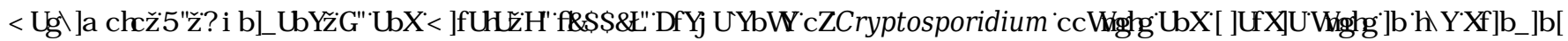

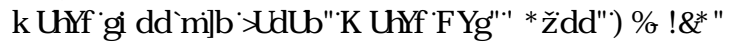

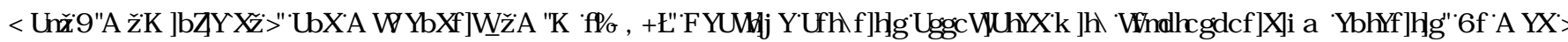

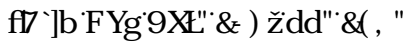

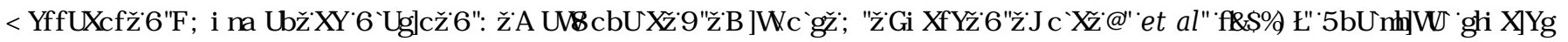

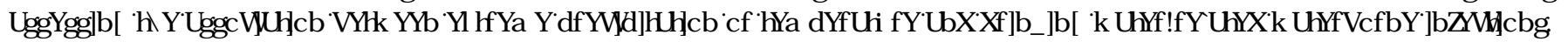

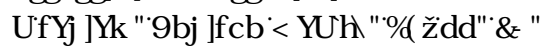

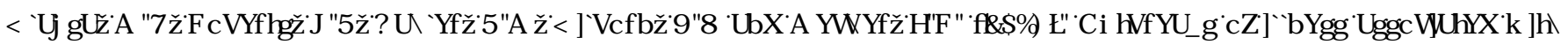

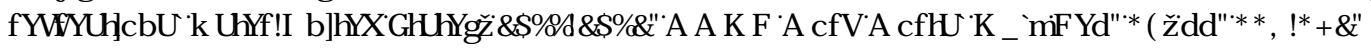




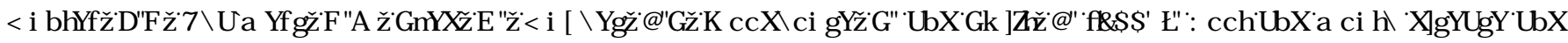

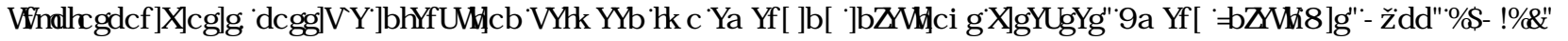

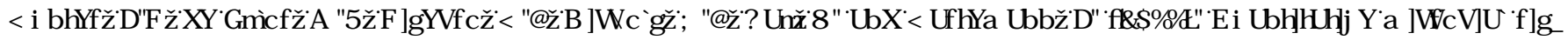

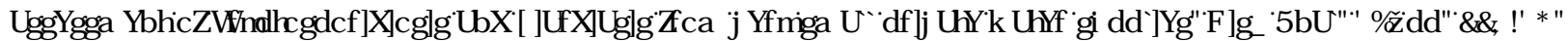

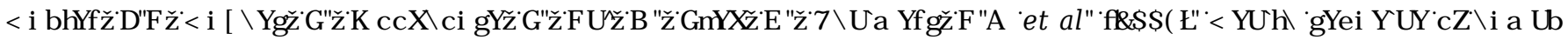
FU SURVSRUGRVVLQTIP P XQRFRP SHMAQWSDMHQW

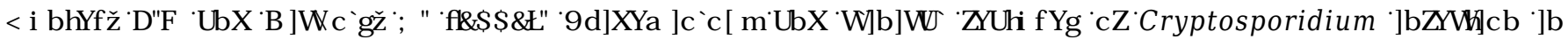

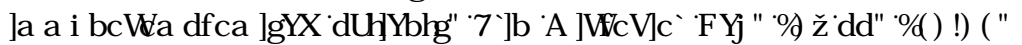

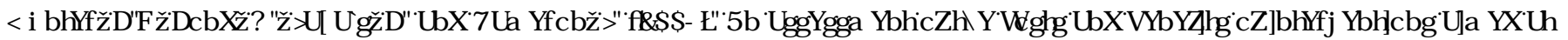

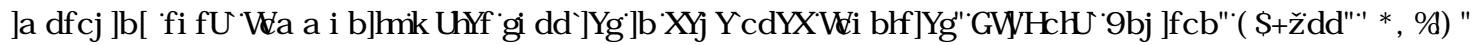

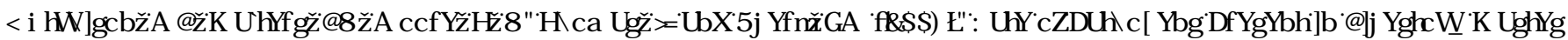

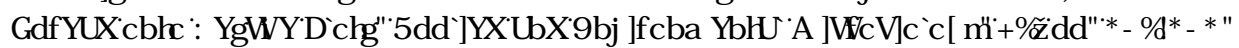

,P UНW.

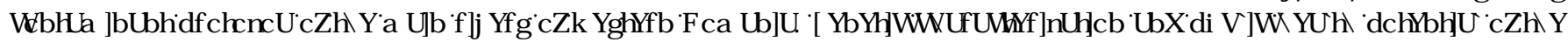

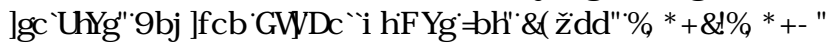

,VDDF[5 HQMRQ

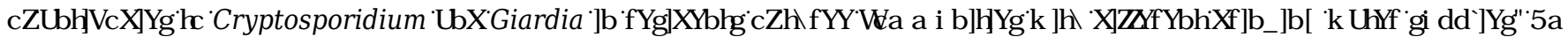

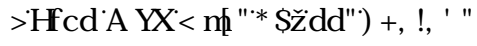

,62

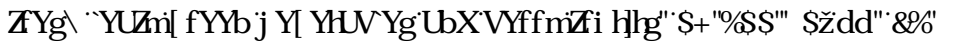

,62 四

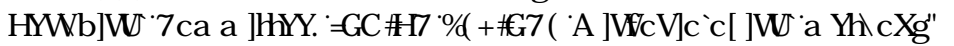

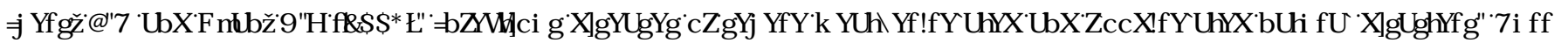

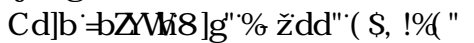

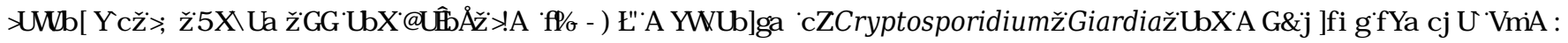

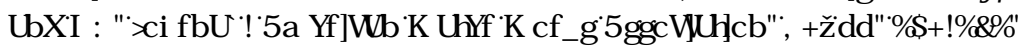

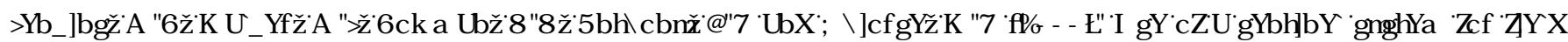

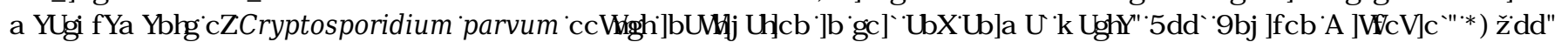

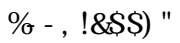

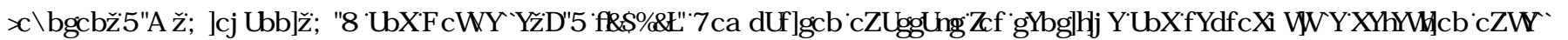

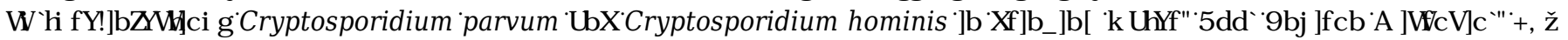
SS

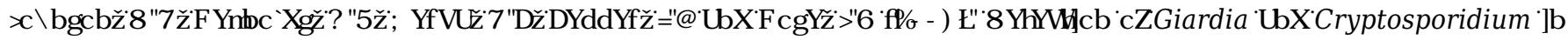

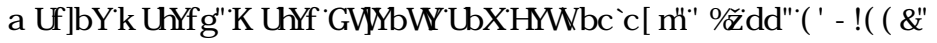

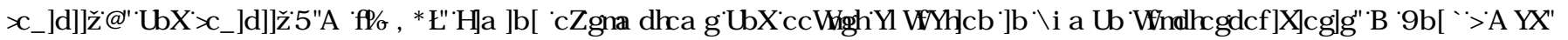

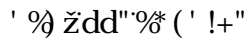

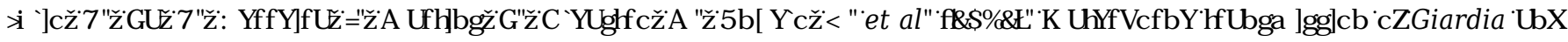
Cryptosporidium [DQUYHUEHDFKHMQ

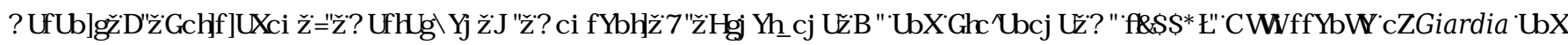

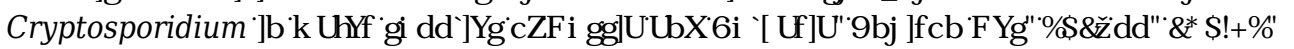




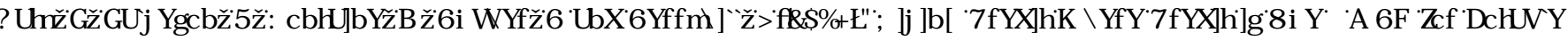

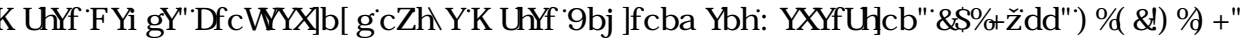

. IU[5

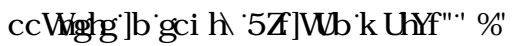

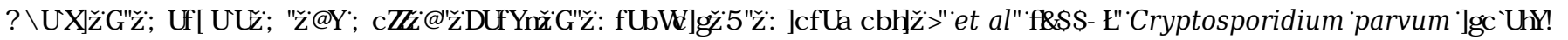

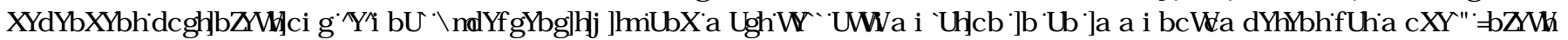

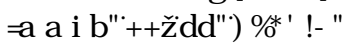

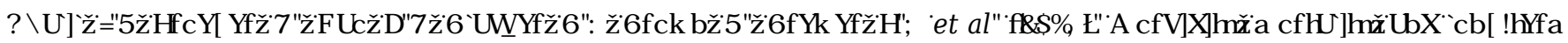

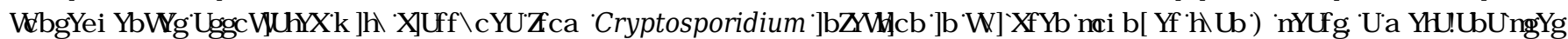

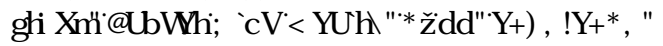

L

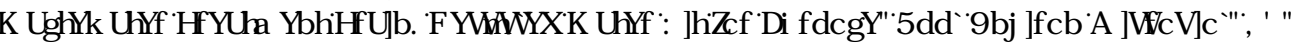

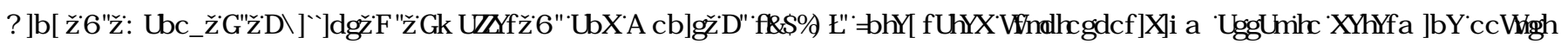

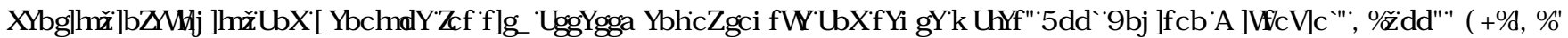

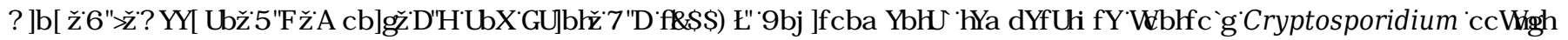

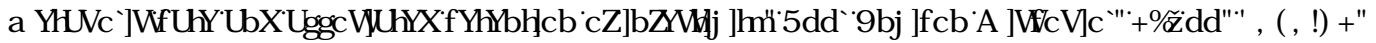

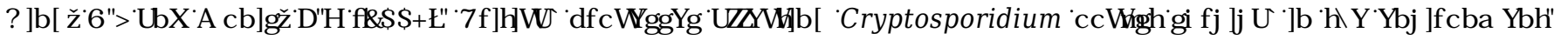

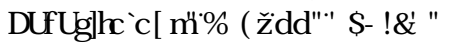

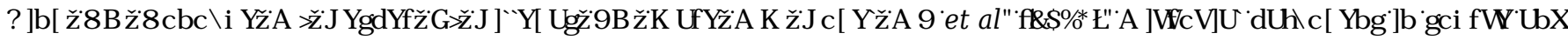

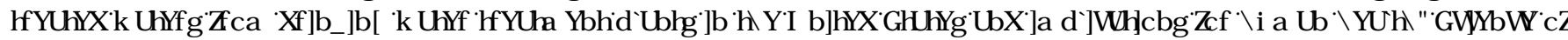

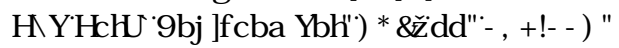

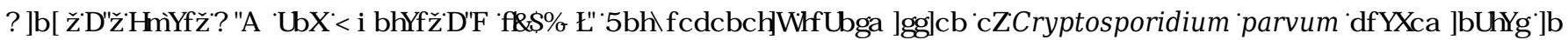

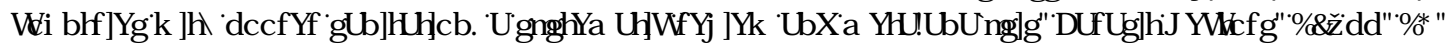

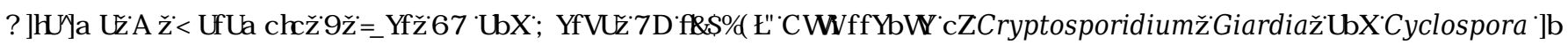

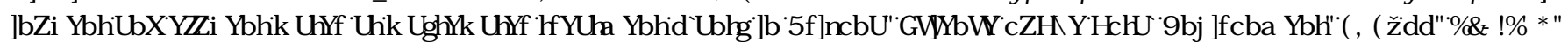

. RUFKM [* [D HDG

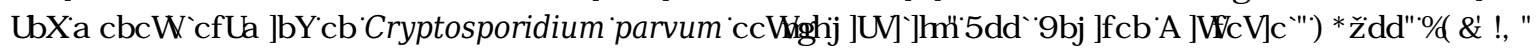

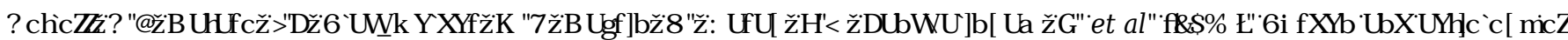

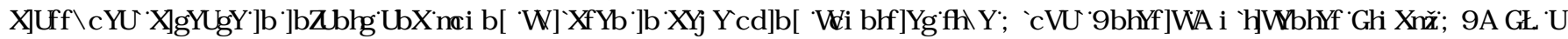

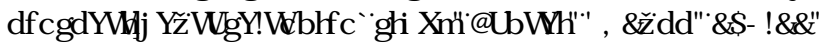

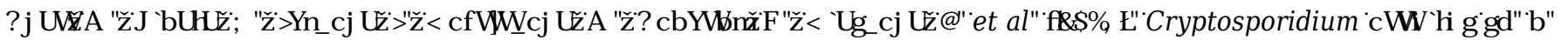

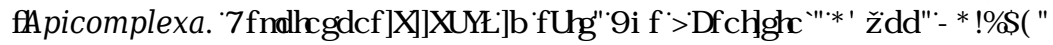

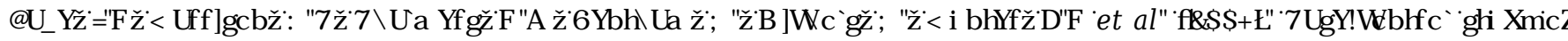

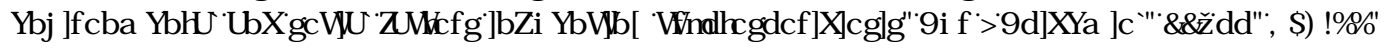

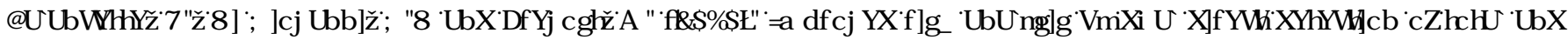

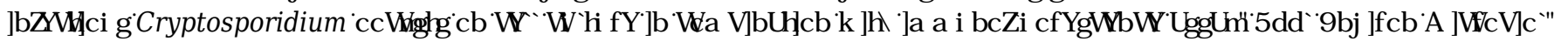

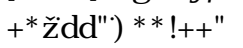

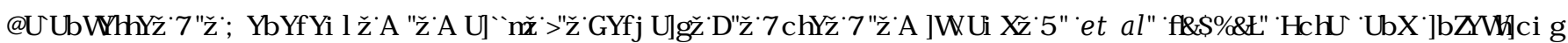

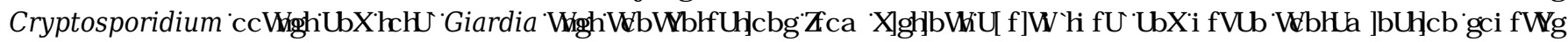

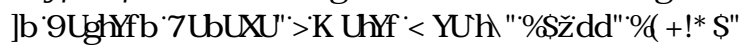




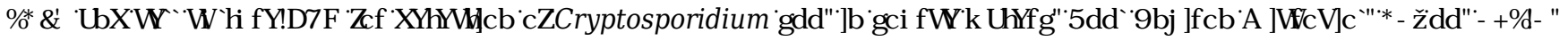

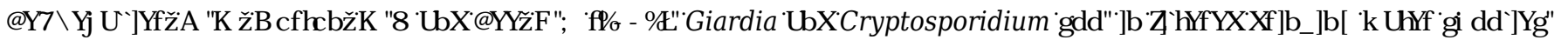

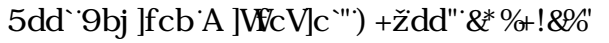

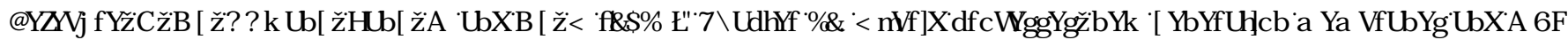

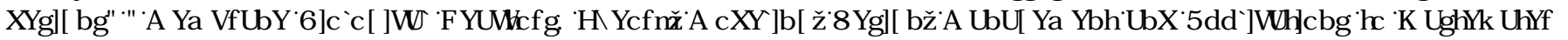

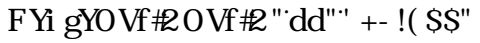

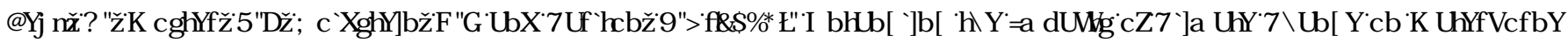

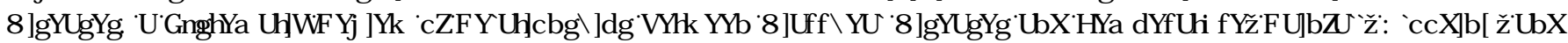

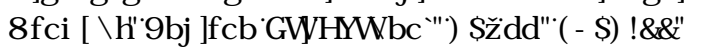

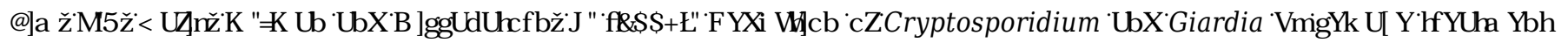

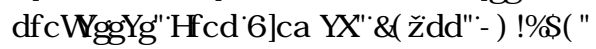

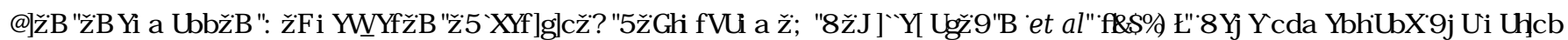

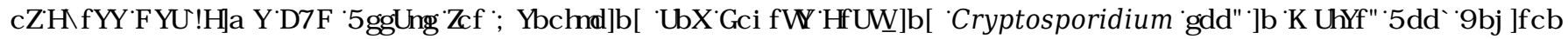

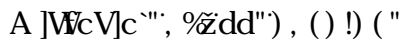

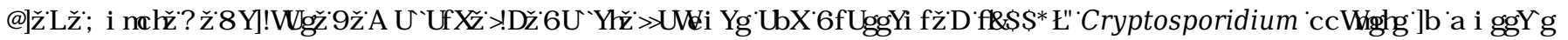

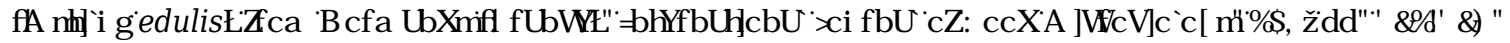

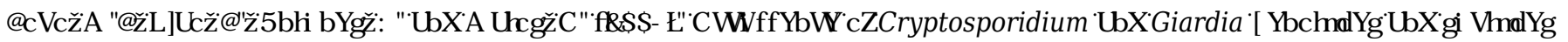

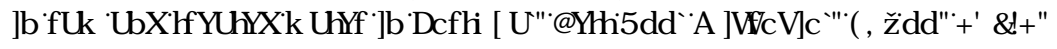

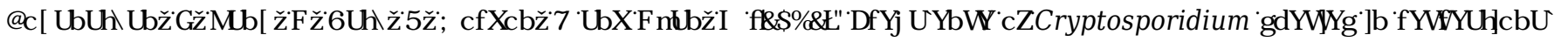

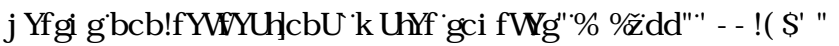

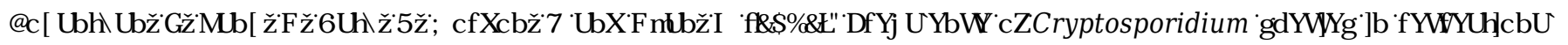

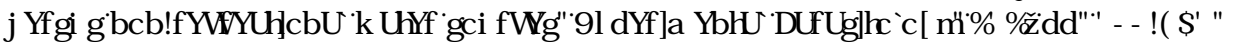

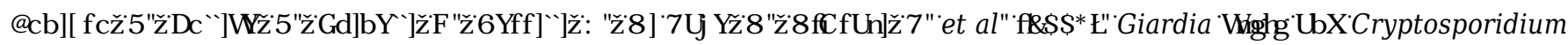

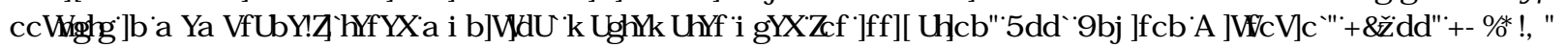

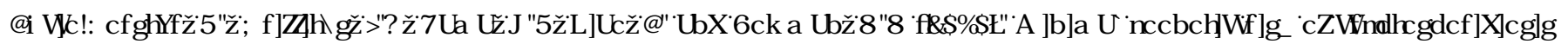

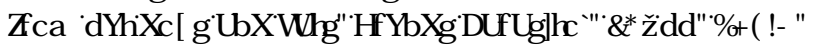

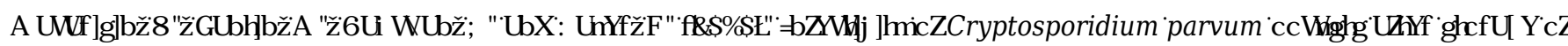

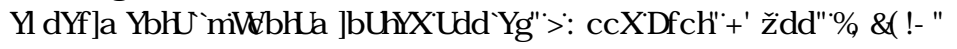

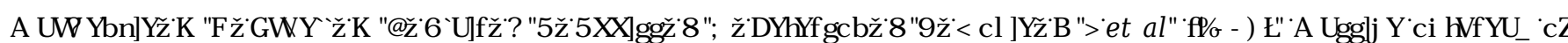

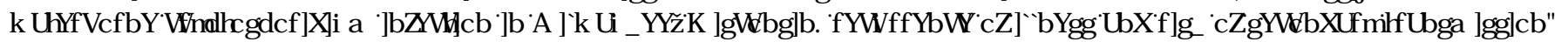
\&QQ

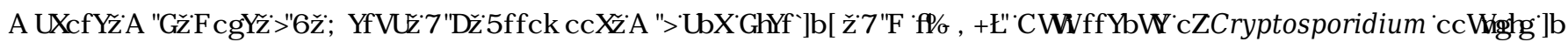

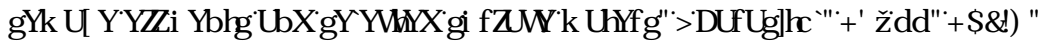

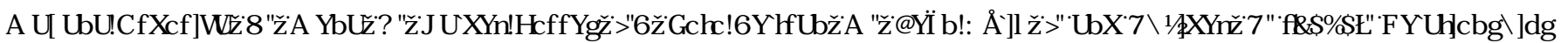

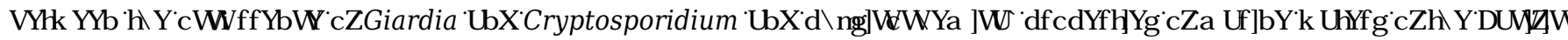

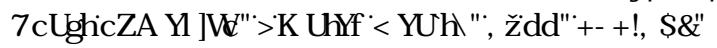

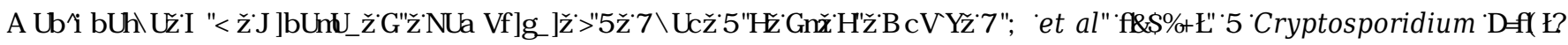

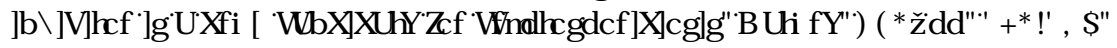

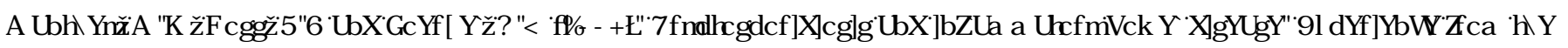

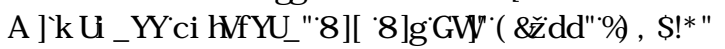




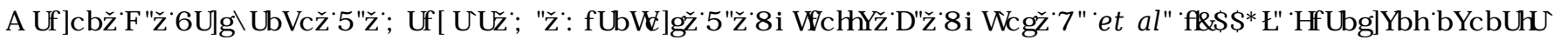

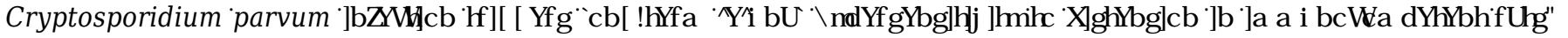
, Q HFVRP P XQ

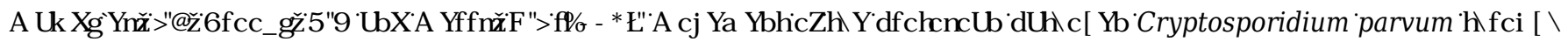

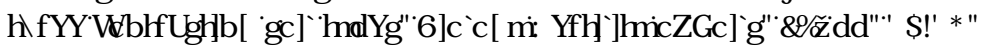

O F\&OCDQMB]

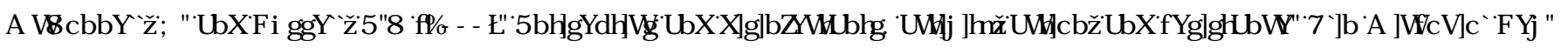

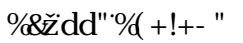

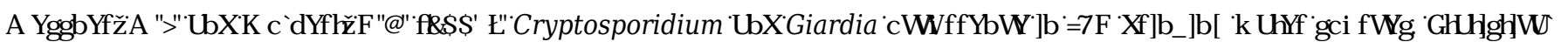

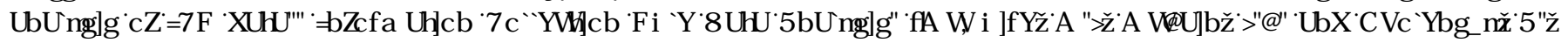
HGW" HOYHUा\&2SS

0 INARYW \% \%

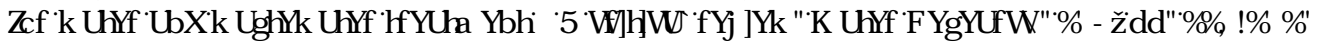

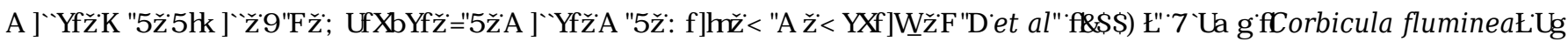

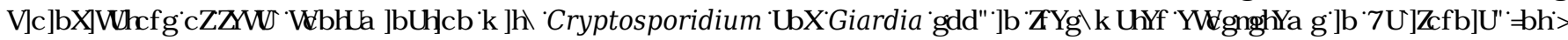

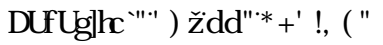

O P ED DIf/

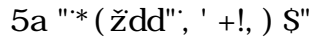

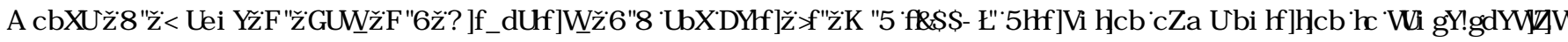

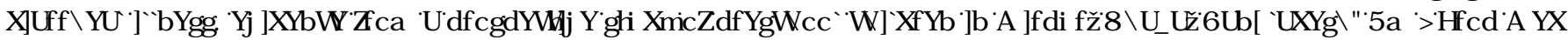

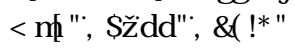

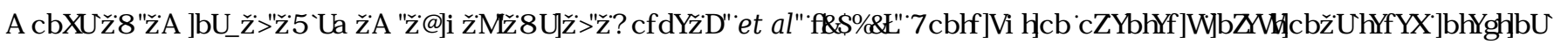

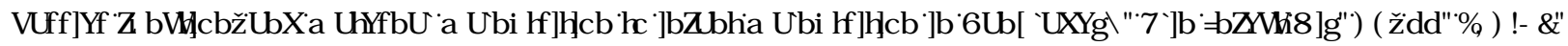

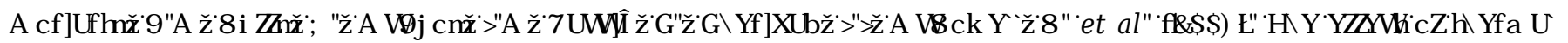

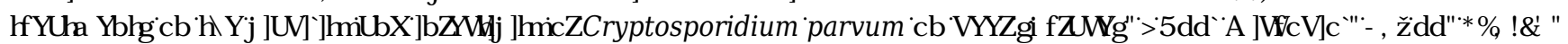

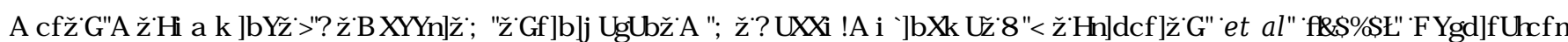

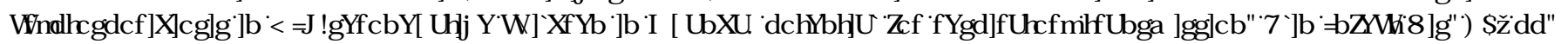
प्रापाला

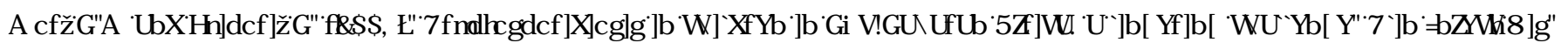

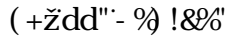

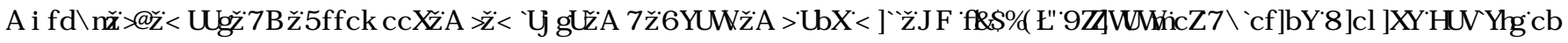

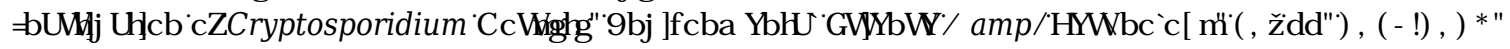

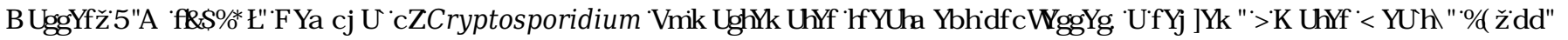

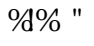

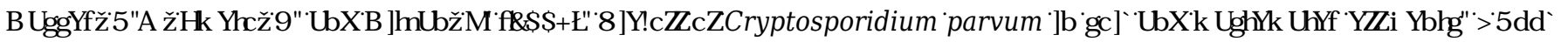

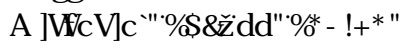

1 DWHUा\$0 四

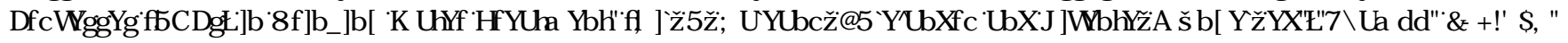

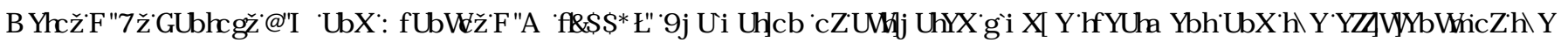

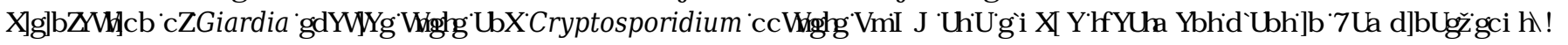

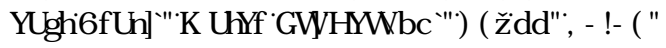


1) XI HQT77KXI Ш

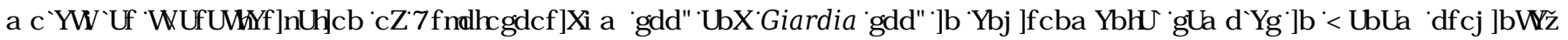

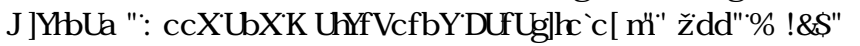

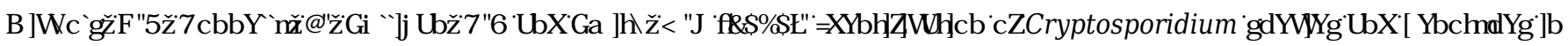

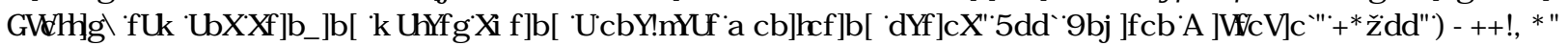

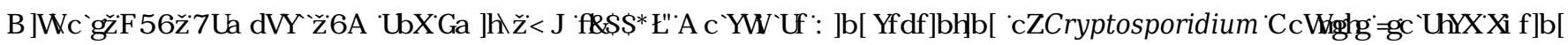

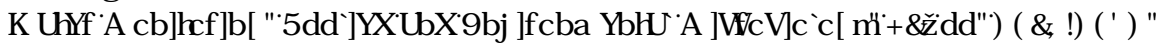

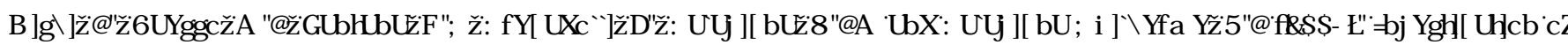

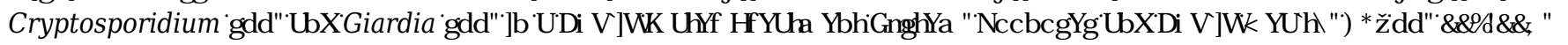

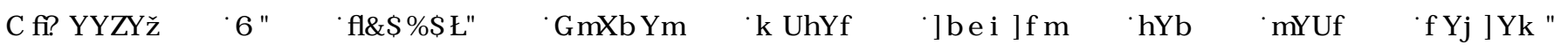

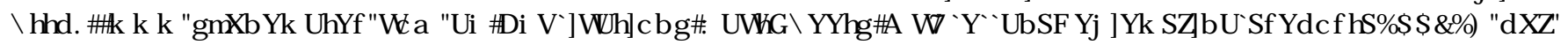

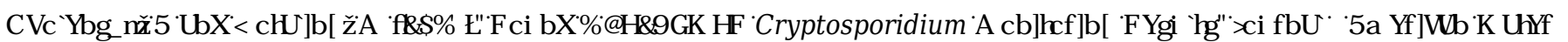

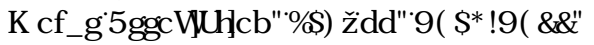

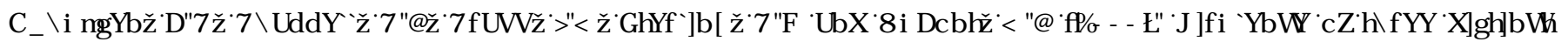

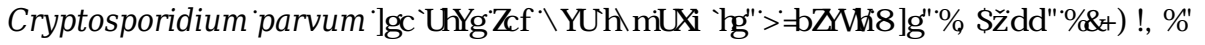

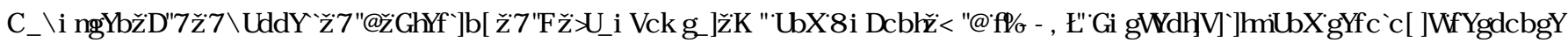

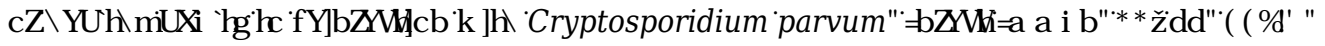

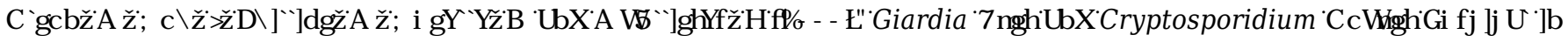

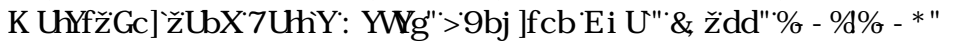

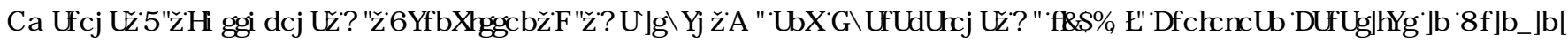

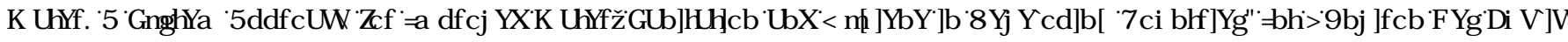

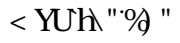

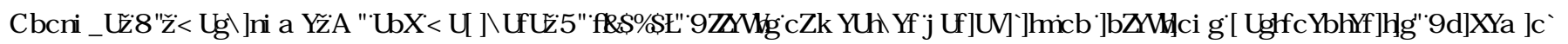

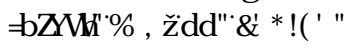

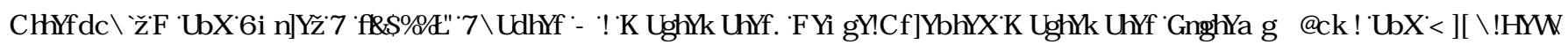

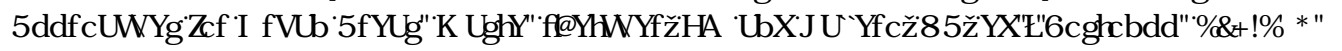

2 WRVRQTH Ш DQMHQT\$

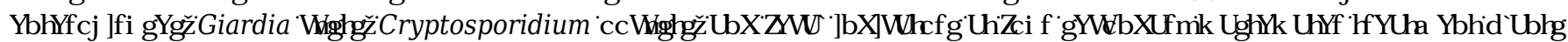

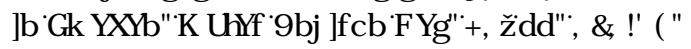

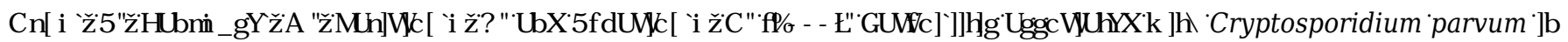

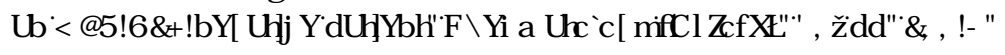

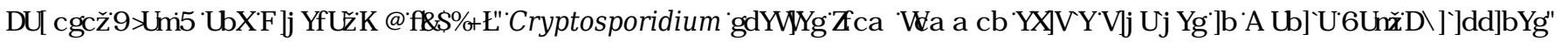

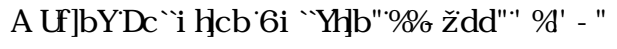

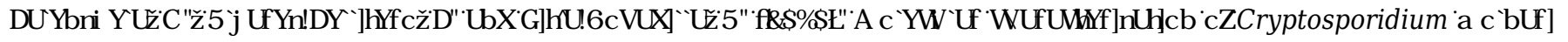

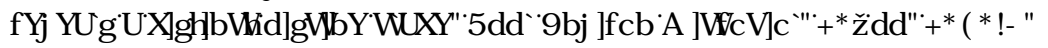

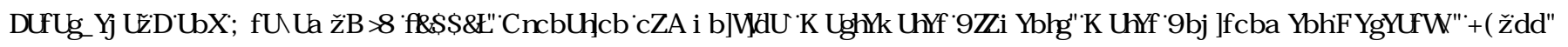
प्राणासए

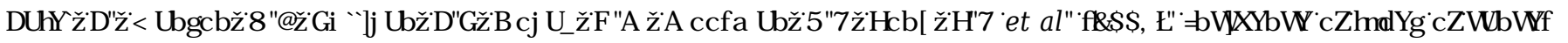

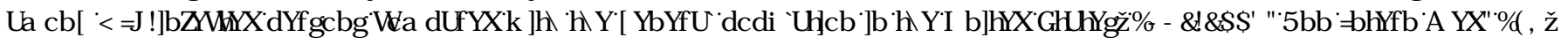
SS 
Pavli, P, Venkateswaran, S, Bradley, M and Bridle, H (2016). Enhancing Cryptosporidium parvum recovery rates for improved water monitoring. Chemosphere. 143, pp. 57-63.

Payment, P., Plante, R. and Cejka, P. (2001). Removal of indicator bacteria, human enteric viruses, Giardia cysts, and Cryptosporidium oocysts at a large wastewater primary treatment facility. Can J Microbiol. 47, pp. 188-93.

Pellegrin, M.L, Aguinaldo, J., Arabi, S., Sadler, M.E, Greiner, A.D, Wong, J. et al. (2018). Membrane Processes. Water Environ Res. 90, pp. 1457-1536.

Peng, X., Murphy, T. and Holden, N.M (2008). Evaluation of the effect of temperature on the die-off rate for Cryptosporidium parvum oocysts in water, soils, and feces. Appl Environ Microbiol. 74, pp. 7101-7.

Petersen, H.H, Enemark, H.L, Olsen, A., Amin, M.G and Dalsgaard, A. (2012). Transport of Cryptosporidium parvum oocysts in soil columns following applications of raw and separated liquid slurries. Appl Environ Microbiol. 78, pp. 5994-6000.

Pinkerton, R., Oria, R.B, Lima, A.A, Rogawski, E.T, Oria, M.O, Patrick, P.D et al. (2016). Early Childhood Diarrhea Predicts Cognitive Delays in Later Childhood Independently of Malnutrition. Am J Trop Med Hyg. 95, pp. 1004-1010.

Plutzer, J., Karanis, P., Domokos, K., Torokne, A. and Marialigeti, K. (2008). Detection and characterisation of Giardia and Cryptosporidium in Hungarian raw, surface and sewage water samples by IFT, PCR and sequence analysis of the SSUrRNA and GDH genes. Int J Hyg Environ Health. 211, pp. 524-33.

Plutzer, J., Tako, M.H, Marialigeti, K., Torokne, A. and Karanis, P. (2007). First investigations into the prevalence of Cryptosporidium and Giardia spp. in Hungarian drinking water. J Water Health. 5, pp. 573-84.

Pokorny, N.J, Weir, S.C, Carreno, R.A, Trevors, J.T and Lee, H. (2002). Influence of temperature on Cryptosporidium parvum oocyst infectivity in river water samples as detected by tissue culture assay. J Parasitol. 88, pp. 641-3.

Pollock, K.G, Young, D., Smith, H.V and Ramsay, C.N (2008). Cryptosporidiosis and filtration of water from Loch Lomond, Scotland. Emerg Infect Dis. 14, pp. 115-20.

Pozio, E. (2004). [Highly Active AntiRetroviral Therapy and opportunistic protozoan infections]. Parassitologia. 46, pp. 89-93.

Pritchard, G.C, Marshall, J.A, Giles, M., Chalmers, R.M and Marshall, R.N (2007). Cryptosporidium parvum infection in orphan lambs on a farm open to the public. Vet Rec. 161, pp. 11-4.

Puleston, R.L, Mallaghan, C.M, Modha, D.E, Hunter, P.R, Nguyen-Van-Tam, J.S, Regan, C.M et al. (2014). The first recorded outbreak of cryptosporidiosis due to Cryptosporidium cuniculus (formerly rabbit genotype), following a water quality incident. J Water Health. 12, pp. 41-50.

Pumipuntu, N. and Piratae, S. (2018). Cryptosporidiosis: A zoonotic disease concern. Vet World. 11, pp. 681-686.

Quinonez-Diaz, M.J, Karpiscak, M.M, Ellman, E.D and Gerba, C.P (2001). Removal of pathogenic and indicator microorganisms by a constructed wetland receiving untreated domestic wastewater. J Environ Sci Health A Tox Hazard Subst Environ Eng. 36, pp. 1311-20.

Quintero-Betancourt, W., Gennaccaro, A.L, Scott, T.M and Rose, J.B (2003). Assessment of methods for detection of infectious Cryptosporidium oocysts and Giardia cysts in reclaimed effluents. Appl Environ Microbiol. 69, pp. 5380-8.

Quintero-Betancourt, W., Peele, E.R and Rose, J.B (2002). Cryptosporidium parvum and Cyclospora cayetanensis: a review of laboratory methods for detection of these waterborne parasites. J Microbiol Methods. 49, pp. 209-24.

Quintero-Betancourt, W and De Ledesma, LBotero (2000). Descriptive study on the presence of protozoan cysts and bacterial indicators in a drinking water treatment plant in Maracaibo, Venezuela. International Journal of Environmental Health Research. 10, pp. 51-61. 
Ramo, A, Del Cacho, E, Sánchez-Acedo, C and Quílez, J (2017). Occurrence and genetic diversity of Cryptosporidium and Giardia in urban wastewater treatment plants in north-eastern Spain. Science of The Total Environment. 598, pp. 628-638.

Razzolini, M.T, Santos, T.F da Silv and Bastos, V.K (2010). Detection of Giardia and Cryptosporidium cysts/oocysts in watersheds and drinking water sources in Brazil urban areas. J Water Health. 8, pp. 399-404.

Rehn, M., Wallensten, A., Widerstrom, M., Lilja, M., Grunewald, M., Stenmark, S. et al. (2015). Post-infection symptoms following two large waterborne outbreaks of Cryptosporidium hominis in Northern Sweden, 2010-2011. BMC Public Health. 15, pp. 529.

Reinoso, R., Blanco, S., Torres-Villamizar, L.A and Bécares, E. (2011). Mechanisms for parasites removal in a waste stabilisation pond. Microb Ecol. 61, pp. 684-92.

Reinoso, R., Bécares, E. and Smith, H.V (2008). Effect of various environmental factors on the viability of Cryptosporidium parvum oocysts. J Appl Microbiol. 104, pp. 980-6.

Reinoso, R., Torres, L.A and Bécares, E. (2008). Efficiency of natural systems for removal of bacteria and pathogenic parasites from wastewater. Sci Total Environ. 395, pp. 80-6.

Rhodes, E.R, Boczek, L.A, Ware, M.W, McKay, M., Hoelle, J.M, Schoen, M. et al. (2015). Determining pathogen and indicator levels in class B municipal organic residuals used for land application. J Environ Qual. 44, pp. 265-74.

Rijal, G., Tolson, J.K, Petropoulou, C., Granato, T.C, Glymph, A., Gerba, C. et al. (2011). Microbial risk assessment for recreational use of the Chicago area waterway system. J Water Health. 9, pp. 169-86.

Rimhanen-Finne, R., Horman, A., Ronkainen, P. and Hanninen, M.L (2002). An IC-PCR method for detection of Cryptosporidium and Giardia in natural surface waters in Finland. J Microbiol Methods. 50, pp. 299-303.

Rimhanen-Finne, R., Vuorinen, A., Marmo, S., Malmberg, S. and Hanninen, M.L (2004). Comparative analysis of Cryptosporidium, Giardia and indicator bacteria during sewage sludge hygienization in various composting processes. Lett Appl Microbiol. 38, pp. 301-5.

Robertson, L.J (2007). The potential for marine bivalve shellfish to act as transmission vehicles for outbreaks of protozoan infections in humans: A review. International Journal of Food Microbiology. 120, pp. 201-216.

Robertson, L.J, Campbell, A.T and Smith, H.V (1992). Survival of Cryptosporidium parvum oocysts under various environmental pressures. Appl Environ Microbiol. 58, pp. 3494-500.

Robertson, L.J and Chalmers, R.M (2013). Foodborne cryptosporidiosis: is there really more in Nordic countries?. Trends Parasitol. 29, pp. 3-9.

Robertson, L.J and Gjerde, B. (2008). Development and use of a pepsin digestion method for analysis of shellfish for Cryptosporidium oocysts and Giardia cysts. J Food Prot. 71, pp. 959-66.

Robertson, L.J and Gjerde, B. (2001). Occurrence of parasites on fruits and vegetables in Norway. J Food Prot. 64, pp. 1793-8.

Robertson, L.J, Hermansen, L. and Gjerde, B.K (2006). Occurrence of Cryptosporidium oocysts and Giardia cysts in sewage in Norway. Appl Environ Microbiol. 72, pp. 5297-303.

Robertson, L.J, Paton, C.A, Campbell, A.T, Smith, P.G, Jackson, M.H, Gilmour, R.A et al. (2000). Giardia cysts and Cryptosporidium oocysts at sewage treatment works in Scotland, UK. Water Research. 34, pp. 2310-2322.

Rochelle, P.A, Upton, S.J, Montelone, B.A and Woods, K. (2005). The response of Cryptosporidium parvum to UV light. Trends Parasitol. 21, pp. 81-7.

Rochelle, P.A and Di Giovanni, G.D (2014). Cryptosporidium oocysts in drinking water and recreational water. Cryptosporidium: parasite and disease. (Cacciò, SM and Widmer, G, ed.).pp. 489-513. 
Rodriguez-Manzano, J., Alonso, J.L, Ferrús, M.A, Moreno, Y., Amorós, I., Calgua, B. et al. (2012). Standard and new faecal indicators and pathogens in sewage treatment plants, microbiological parameters for improving the control of reclaimed water. Water Sci Technol. 66, pp. 2517-23.

Rosado-Garcia, F.M, Guerrero-Florez, M., Karanis, G., Hinojosa, M.DC and Karanis, P. (2017). Water-borne protozoa parasites: The Latin American perspective. Int J Hyg Environ Health. 220, pp. 783-798.

Rose, J.B (1997). Environmental ecology of Cryptosporidium and public health implications. Annu Rev Public Health. 18, pp. 135-61.

Rose, JB, Huffman, DE, Riley, K, Farrah, SR, Lukasik, JO and Hamann, CL (2001). Reduction of Enteric Microorganisms at the Upper Occoquan Sewage Authority Water Reclamation Plant. Water Environment Research. 73, pp. 711-720.

Ruecker, N.J, Matsune, J.C, Lapen, D.R, Topp, E., Edge, T.A and Neumann, N.F (2013). The detection of Cryptosporidium and the resolution of mixtures of species and genotypes from water. Infect Genet Evol. 15, pp. 3-9.

Ruggiero, M.A, Gordon, D.P, Orrell, T.M, Bailly, N., Bourgoin, T., Brusca, R.C et al. (2015). Correction: A Higher Level Classification of All Living Organisms. PLoS One. 10, pp. e0130114.

Ryan, U., Fayer, R. and Xiao, L. (2014). Cryptosporidium species in humans and animals: current understanding and research needs. Parasitology. 141, pp. 1667-85.

Ryan, U., Hijjawi, N. and Xiao, L. (2018). Foodborne cryptosporidiosis. Int J Parasitol. 48, pp. 1-12.

Ryan, U., Paparini, A., Monis, P. and Hijjawi, N. (2016). It's official - Cryptosporidium is a gregarine: What are the implications for the water industry?. Water Res. 105, pp. 305-313.

Rzezutka, A., Nichols, R.A, Connelly, L., Kaupke, A., Kozyra, I., Cook, N. et al. (2010). Cryptosporidium oocysts on fresh produce from areas of high livestock production in Poland. Int J Food Microbiol. 139, pp. 96-101.

Saeed, T and Sun, G (2012). A review on nitrogen and organics removal mechanisms in subsurface flow constructed wetlands: Dependency on environmental parameters, operating conditions and supporting media. Journal of Environmental Management. 112, pp. 429-448.

Sampson, A, Owusu-Ansah, Ede-Graft J, Mills-Robertson, FC, Ayi, I, Abaidoo, RC, Hald, T et al. (2017). Probabilistic quantitative microbial risk assessment model of farmer exposure to Cryptosporidium spp. in irrigation water within Kumasi Metropolis-Ghana. Microbial Risk Analysis. 6, pp. 1-8.

Santin, M. (2013). Clinical and subclinical infections with Cryptosporidium in animals. N Z Vet J. 61, pp. 1-10.

Santin, M., Trout, J.M, Xiao, L., Zhou, L., Greiner, E. and Fayer, R. (2004). Prevalence and age-related variation of Cryptosporidium species and genotypes in dairy calves. Vet Parasitol. 122, pp. 103-17.

Santos, P.R and Daniel, L.A (2017). Occurrence and removal of Giardia spp. cysts and Cryptosporidium spp. oocysts from a municipal wastewater treatment plant in Brazil. Environ Technol. 38, pp. 1245-1254.

Sarkar, R., Ajjampur, S.S, Prabakaran, A.D, Geetha, J.C, Sowmyanarayanan, T.V, Kane, A. et al. (2013). Cryptosporidiosis among children in an endemic semiurban community in southern India: does a protected drinking water source decrease infection?. Clin Infect Dis. 57, pp. 398-406.

Sato, M.I, Galvani, A.T, Padula, J.A, Nardocci, A.C, S. Mde, L, Razzolini, M.T et al. (2013). Assessing the infection risk of Giardia and Cryptosporidium in public drinking water delivered by surface water systems in Sao Paulo State, Brazil. Sci Total Environ. 442, pp. 389-96.

Savioli, L., Smith, H. and Thompson, A. (2006). Giardia and Cryptosporidium join the 'Neglected Diseases Initiative'. Trends Parasitol. 22, pp. 203-8.

Schets, F.M, van den Berg, H.H, Engels, G.B, Lodder, W.J and Husman, A.M de Roda (2007). Cryptosporidium and Giardia 
in commercial and non-commercial oysters (Crassostrea gigas) and water from the Oosterschelde, The Netherlands. Int J Food Microbiol. 113, pp. 189-94.

Schets, F.M, van Wijnen, J.H, Schijven, J.F, Schoon, H. and Husman, A.M de Roda (2008). Monitoring of waterborne pathogens in surface waters in amsterdam, the Netherlands, and the potential health risk associated with exposure to cryptosporidium and giardia in these waters. Appl Environ Microbiol. 74, pp. 2069-78.

Schmitz, B, Moriyama, H, Haramoto, E, Kitajima, M, Sherchan, S, Gerba, C et al. (2018). Reduction of Cryptosporidium, Giardia, and Fecal Indicators by Bardenpho Wastewater Treatment. Environ Sci Technol. 52, pp. 7015-7023.

Scott, T, McLaughlin, M.R, Harwood, V, Chivukula, V, Levine, A, Gennaccaro, A. et al. (2004). Reduction of pathogens, indicator bacteria, and alternative indicators by wastewater treatment and reclamation processes. Wat Sci Tech \&amp; Tech Wat Suppl. 3, pp. 247-252.

Shebl, F.M, Engels, E.A and Goedert, J.J (2012). Opportunistic intestinal infections and risk of colorectal cancer among people with AIDS. AIDS Res Hum Retroviruses. 28, pp. 994-99.

Sheludchenko, M., Padovan, A., Katouli, M. and Stratton, H. (2016). Removal of Fecal Indicators, Pathogenic Bacteria, Adenovirus, Cryptosporidium and Giardia (oo)cysts in Waste Stabilization Ponds in Northern and Eastern Australia. Int J Environ Res Public Health. 13.

Shepherd, R.C, Smail, P.J and Sinha, G.P (1989). Reactive arthritis complicating cryptosporidial infection. Arch Dis Child. 64, pp. 743-4.

Shirley, D.A, Moonah, S.N and Kotloff, K.L (2012). Burden of disease from cryptosporidiosis. Curr Opin Infect Dis. 25, pp. 555-63.

Shrivastava, A.K, Kumar, S., Smith, W.A and Sahu, P.S (2017). Revisiting the global problem of cryptosporidiosis and recommendations. Trop Parasitol. 7, pp. 8-17.

Shuval, H. (2003). Estimating the global burden of thalassogenic diseases: Human infectious diseases caused by wastewater pollution of the marine environment. Journal of Water and Health. 1, pp. 53-64.

Slifko, T.R, Huffman, D.E, Dussert, B., Owens, J.H, Jakubowski, W., Haas, C.N et al. (2002). Comparison of tissue culture and animal models for assessment of Cryptospridium parvum infection. Exp Parasitol. 101, pp. 97-106.

Smeets, P.W, van Dijk, J.C, Stanfield, G., Rietveld, L.C and Medema, G.J (2007). How can the UK statutory Cryptosporidium monitoring be used for Quantitative Risk Assessment of Cryptosporidium in drinking water?. J Water Health. 5 Suppl 1, pp. 107-18.

Sow, S.O, Muhsen, K., Nasrin, D., Blackwelder, W.C, Wu, Y., Farag, T.H et al. (2016). The Burden of Cryptosporidium Diarrheal Disease among Children < 24 Months of Age in Moderate/High Mortality Regions of Sub-Saharan Africa and South Asia, Utilizing Data from the Global Enteric Multicenter Study (GEMS). PLoS Negl Trop Dis. 10, pp. e0004729.

Sponseller, J.K, Griffiths, J.K and Tzipori, S. (2014). The evolution of respiratory Cryptosporidiosis: evidence for transmission by inhalation. Clin Microbiol Rev. 27, pp. 575-86.

Sroka, J., Stojecki, K., Zdybel, J., Karamon, J., Cencek, T. and Dutkiewicz, J. (2013). Occurrence of Cryptosporidium oocysts and Giardia cysts in effluent from sewage treatment plant from eastern Poland. Ann Agric Environ Med. Spec no. 1, pp. 57-62.

Stadterman, K.L, Sninsky, A.M, Sykom, J.L and Jakubowski, W. (1995). Removal and inactivation of Cryptosporidium oocysts by activated sludge treatment and anaerobic digestion. Water Science and Technology. 31, pp. 97-104.

Staggs, S.E, Beckman, E.M, Keely, S.P, Mackwan, R., Ware, M.W, Moyer, A.P et al. (2013). The Applicability of TaqManBased Quantitative Real-Time PCR Assays for Detecting and Enumerating Cryptosporidium spp. Oocysts in the Environment. PLoS One. 8, pp. e66562. 
Staggs, S.E, Keely, S.P, Ware, M.W, Schable, N., See, M.J, Gregorio, D. et al. (2015). The development and implementation of a method using blue mussels (Mytilus spp.) as biosentinels of Cryptosporidium spp. and Toxoplasma gondii contamination in marine aquatic environments. Parasitol Res. 114, pp. 4655-67.

Steele, M and Odumeru, J (2005). Irrigation water as source of foodborne pathogens on fruit and vegetables. Journal of Food Protection, 67(12), 2839-2849. 67, pp. 2839-49.

Stott, R., May, E., Matsushita, E. and Warren, a. (2001). Protozoan predation as a mechanism for the removal of cryptosporidium oocysts from wastewaters in constructed wetlands. Water Sci Technol. 44, pp. 191-8.

Stott, R., May, E., Ramirez, E. and Warren, a. (2003). Predation of Cryptosporidium oocysts by protozoa and rotifers: implications for water quality and public health. Water Sci Technol. 47, pp. 77-83.

Sulzyc-Bielicka, V., Kolodziejczyk, L., Jaczewska, S., Bielicki, D., Kladny, J. and Safranow, K. (2012). Prevalence of Cryptosporidium sp. in patients with colorectal cancer. Pol Przegl Chir. 84, pp. 348-51.

Sulzyc-Bielicka, V., Kuzna-Grygiel, W., Kolodziejczyk, L., Bielicki, D., Kladny, J., Stepien-Korzonek, M. et al. (2007). Cryptosporidiosis in patients with colorectal cancer. J Parasitol. 93, pp. 722-4.

Sunnotel, O., Verdoold, R., Dunlop, P.S, Snelling, W.J, Lowery, C.J, Dooley, J.S et al. (2010). Photocatalytic inactivation of Cryptosporidium parvum on nanostructured titanium dioxide films. J Water Health. 8, pp. 83-91.

Suppes, LM, Canales, RA, Gerba, CP and Reynolds, KA (2016). Cryptosporidium risk from swimming pool exposures. International Journal of Hygiene and Environmental Health. 219, pp. 915-919.

Sutthikornchai, C, Popruk, S, Chumpolbanchorn, K, Sukhumavasi, W and Sukthana, Y (2016). Oyster is an effective transmission vehicle for Cryptosporidium infection in human. Asian Pacific Journal of Tropical Medicine. 9, pp. 562-566.

Taran-Benshoshan, M., Ofer, N., Dalit, V.O, Aharoni, A., Revhun, M., Nitzan, Y. et al. (2015). Cryptosporidium and Giardia removal by secondary and tertiary wastewater treatment. J Environ Sci Health A Tox Hazard Subst Environ Eng. 50, pp. 1265-73.

Thurston-Enriquez, JA, Watt, P, Dowd, SE, Enriquez, R, Pepper, IL and Gerba, CP (2002). Detection of Protozoan Parasites and Microsporidia in Irrigation Waters Used for Crop Production. Journal of Food Protection. 65, pp. 378-382.

Thurston, JA, Foster, KE, Karpiscak, MM and Gerba, CP (2001). Fate of indicator microorganisms, giardia and cryptosporidium in subsurface flow constructed wetlands. Water Research. 35, pp. 1547-1551.

Tomizawa, D., Imai, K., Ito, S., Kajiwara, M., Minegishi, Y., Nagasawa, M. et al. (2004). Allogeneic hematopoietic stem cell transplantation for seven children with X-linked hyper-IgM syndrome: a single center experience. Am J Hematol. 76, pp. 33-9.

Tondera, K., Klaer, K., Gebhardt, J., Wingender, J., Koch, C., Horstkott, M. et al. (2015). Reducing pathogens in combined sewer overflows using ozonation or UV irradiation. Int J Hyg Environ Health. 218, pp. 731-41.

Turtoi, M. (2013). Ultraviolet light potential for wastewater disinfection. Ann. Food Sci. Technol.. 14, pp. 153-164.

Uga, S., Matsuo, J., Kono, E., Kimura, K., Inoue, M., Rai, S.K et al. (2000). Prevalence of Cryptosporidium parvum infection and pattern of oocyst shedding in calves in Japan. Vet Parasitol. 94, pp. 27-32.

USEPA (2012). Method 1623.1: Cryptosporidium and Giardia in Water by Filtration/IMS/FA. EPA 816-R-12-001, Washington, DC

USEPA (2005). Method 1623: Cryptosporidium and Giardia in water by filtration/IMS/FA. EPA 815-R-05-002, Washington, $\mathrm{DC}$

USEPA, (2000). National primary drinking water regulations: long-term 2 enhanced surface water treatment rule. Proposed Rule. Fed. Reg. . 68. 
Valentiner-Branth, P., Steinsland, H., Fischer, T.K, Perch, M., Scheutz, F., Dias, F. et al. (2003). Cohort study of Guinean children: incidence, pathogenicity, conferred protection, and attributable risk for enteropathogens during the first 2 years of life. J Clin Microbiol. 41, pp. 4238-45.

Verbyla, M., von Sperling, M. and Maiga, Y. (2017). Waste Stabilization Ponds. Global Water Pathogens Project. (Rose, JB, Jiménez-Cisneros, B, Haas, C., and Verbyla, ME, ed.).East Lansing, Michigan, USA doi: https://doi.org/10.14321/waterpathogens.65.

Vermeulen, L.C, Benders, J., Medema, G. and Hofstra, N. (2017). Global Cryptosporidium Loads from Livestock Manure. Environ Sci Technol. 51, pp. 8663-8671.

Wallis, P.M, Erlandsen, S.L, Isaac-Renton, J.L, Olson, M.E, Robertson, W.J and van Keulen, H. (1996). Prevalence of Giardia cysts and Cryptosporidium oocysts and characterization of Giardia spp. isolated from drinking water in Canada. Appl Environ Microbiol. 62, pp. 2789-97.

Weber, R., Bryan, R.T, Bishop, H.S, Wahlquist, S.P, Sullivan, J.J and Juranek, D.D (1991). Threshold of detection of Cryptosporidium oocysts in human stool specimens: evidence for low sensitivity of current diagnostic methods. J Clin Microbiol. 29, pp. 1323-7.

Wells, B., Shaw, H., Hotchkiss, E., Gilray, J., Ayton, R., Green, J. et al. (2015). Prevalence, species identification and genotyping Cryptosporidium from livestock and deer in a catchment in the Cairngorms with a history of a contaminated public water supply. Parasit Vectors. 8, pp. 66.

Whitmore, T.N and Robertson, L.J (1995). The effect of sewage-sludge treatment processes on oocysts of Cryptosporidiumparvum. Journal of Applied Bacteriology. 78, pp. 34-38.

Willis, JE, McClure, J.T, Davidson, J, McClure, C and Greenwood, SJ (2013). Global occurrence of Cryptosporidium and Giardia in shellfish: Should Canada take a closer look?. Food Research International. 52, pp. 119-135.

Wilson, L, Anthony, S, Kay, D. and Procter, C (2008). Evaluation and development of Cryptosporidium risk assessment. (ADA/012/07, SGovernment, ed.).

Wojtenko, I, Stinson, MK and Field, R (2001). Performance of Ozone as a Disinectant for Combined Sewer Overflow. Critical Reviews in Environmental Science and Technology. 31, pp. 295-309.

Xiao, L. (2010). Molecular epidemiology of cryptosporidiosis: an update. Exp Parasitol. 124, pp. 80-9.

Xiao, L. and Fayer, R. (2008). Molecular characterisation of species and genotypes of Cryptosporidium and Giardia and assessment of zoonotic transmission. Int J Parasitol. 38, pp. 1239-55.

Xiao, L. and Feng, Y. (2008). Zoonotic cryptosporidiosis. FEMS Immunol Med Microbiol. 52, pp. 309-23.

Xiao, L., Lal, A.A and Jiang, J. (2004). Detection and differentiation of Cryptosporidium oocysts in water by PCR-RFLP. Methods Mol Biol. 268, pp. 163-76.

Xiao, L., Singh, A., Limor, J., Graczyk, T.K, Gradus, S. and Lal, A. (2001). Molecular characterization of cryptosporidium oocysts in samples of raw surface water and wastewater. Appl Environ Microbiol. 67, pp. 1097-101.

Xiao, L., Zhou, L., Santin, M., Yang, W. and Fayer, R. (2007). Distribution of Cryptosporidium parvum subtypes in calves in eastern United States. Parasitol Res. 100, pp. 701-6.

Xiao, S., Hu, S., Zhang, Y., Zhao, X. and Pan, W. (2018). Influence of sewage treatment plant effluent discharge into multipurpose river on its water quality: A quantitative health risk assessment of Cryptosporidium and Giardia. Environ Pollut. 233, pp. 797-805.

Xiao, S., Zhang, Y., Zhao, X., Sun, L. and Hu, S. (2018). Presence and molecular characterization of Cryptosporidium and Giardia in recreational lake water in Tianjin, China: a preliminary study. Sci Rep. 8, pp. 2353. 
Xiao, S, Sike, hu, Zhang, Y, Zhao, X and Pan, W (2017). Influence of sewage treatment plant effluent discharge into multipurpose river on its water quality: A quantitative health risk assessment of Cryptosporidium and Giardia. 233, pp. 797-805.

Yu, Z. and Mohn, W.W (2001). Bacterial diversity and community structure in an aerated lagoon revealed by ribosomal intergenic spacer analyses and 16S ribosomal DNA sequencing. Appl Environ Microbiol. 67, pp. 1565-74.

Zahedi, A., Durmic, Z., Gofton, A.W, Kueh, S., Austen, J., Lawson, M. et al. (2017). Cryptosporidium homai n. sp. (Apicomplexa: Cryptosporidiiae) from the guinea pig (Cavia porcellus). Vet Parasitol. 245, pp. 92-101.

Zahedi, A., Lee, G.KC, Greay, T.L, Walsh, A.L, Blignaut, D.JC and Ryan, U.M (2018). First report of Cryptosporidium parvum in a dromedary camel calf from Western Australia. Acta Parasitol. 63, pp. 422-427.

Zahedi, A., Paparini, A., Jian, F., Robertson, I. and Ryan, U. (2016). Public health significance of zoonotic Cryptosporidium species in wildlife: Critical insights into better drinking water management. Int J Parasitol Parasites Wildl. 5, pp. 88-109.

Zahedi, A, Monis, P, Gofton, AW, Oskam, CL, Ball, A, Bath, A et al. (2018). Cryptosporidium species and subtypes in animals inhabiting drinking water catchments in three states across Australia. Water Research. 134, pp. 327-340.

Zhang, T., Hu, H.Y, Zong, Z.S and Xie, X. (2008). [Removal characteristic of pathogenic protozoan in wastewater treatment and reclamation process]. Huan Jing Ke Xue. 29, pp. 1955-60.

Zhang, Y, Rottiers, T, Meesschaert, B, Pinoy, L and Van der Bruggen, B (2019). Chapter 1 - Wastewater Treatment by Renewable Energy Driven Membrane Processes. Current Trends and Future Developments on (Bio-) Membranes. (Basile, A, Cassano, A and Figoli, A, ed.).pp. 1-19. 